\title{
Integral Menger Curvature and Rectifiability of $n$-dimensional Borel sets in Euclidean $\mathrm{N}$-space
}

Von der Fakultät für Mathematik, Informatik und Naturwissenschaften der

RWTH Aachen University zur Erlangung des akademischen Grades eines Doktors der Naturwissenschaften genehmigte Dissertation

vorgelegt von

Diplom-Mathematiker

Martin Meurer

aus Viersen

Berichter: Univ.-Prof. Dr. Heiko von der Mosel

Priv.-Doz. Dr. Alfred Wagner

Tag der mündlichen Prüfung: 10. April 2015

Diese Dissertation ist auf den Internetseiten der Hochschulbibliothek online verfügbar. 



\section{Abstract}

In this thesis we show that an $n$-dimensional Borel set in Euclidean $N$-space with finite integral Menger curvature is $n$-rectifiable, meaning that it can be covered by countably many images of Lipschitz continuous functions up to a null set in the sense of Hausdorff measure. This generalises Léger's [Lég99] rectifiability result for one-dimensional sets to arbitrary dimension and co-dimension. In addition, we characterise possible integrands and discuss examples known from the literature.

Intermediate results of independent interest include upper bounds of different versions of P. Jones's $\beta$-numbers in terms of integral Menger curvature without assuming lower Ahlfors regularity, in contrast to the results of Lerman and Whitehouse [LW09]. 



\section{Zusammenfassung}

In dieser Arbeit zeigen wir, dass eine $n$-dimensionale Borel Menge mit endlicher integraler Menger Krümmung $n$-rektifizierbar ist, d.h. es existieren abzählbar viele Lipschitz Abbildungen, deren Bilder, bis auf eine Nullmenge bzgl. des Hausdorff Maßes, die Menge überdecken. Dies ist eine Verallgemeinerung von Légers Arbeit [Lég99] über Rektifizierbarkeit von eindimensionalen Mengen zu Mengen beliebiger Dimension und Codimension. Wir charakterisieren mögliche Integranden und diskutieren einige bekannte Beispiele aus anderen Arbeiten.

Als Zwischenergebnis zeigen wir Abschätzungen von P. Jones $\beta$-Zahlen gegen die integrale Menger Krümmung. Im Gegensatz zu den Arbeiten von Lerman and Whitehouse [LW09] müssen wir nur obere Ahlfors Regularität voraussetzen. 



\section{Acknowledgements}

I would like to express my deep gratitude to my supervisor Prof. Dr. Heiko von der Mosel for his continuous and precious advice. I would like to thank PD Dr. Alfred Wagner for accepting to be the second referee. Special thanks go to my colleagues at the Institut für Mathematik for fruitful discussions, particularly to Dr. Armin Schikorra, Dr. Sebastian Scholtes, Dr. Patrik Overath and all members and former members of office 118.1. I am very thankful to Stephanie Feddern for proofreading this thesis.

Last but not least, I'm very grateful to Christina, Anna and my family for their support and care. 



\section{Contents}

1 Introduction 1

2 Preliminaries $\quad 7$

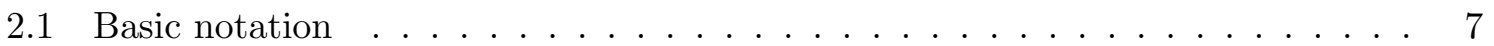

2.2 Basic linear algebra facts . . . . . . . . . . . . . . . . . . 7

2.3 Simplices . . . . . . . . . . . . . . . . . . . 10

2.4 Angles between affine subspaces . . . . . . . . . . . . . . . . . . . 17

3 Integral Menger curvature and rectifiability $\quad 27$

3.1 Main result . . . . . . . . . . . . . . . . . 27

3.2 Examples of admissible integrands . . . . . . . . . . . . 28

4 Proof of the main result $\quad 35$

4.1 Reduction to a symmetric integrand . . . . . . . . . . . . . 35

4.2 Reduction to finite, compact and more regular sets with small curvature . . . . 35

$5 \beta$-numbers $\quad \mathbf{4 5}$

5.1 Measure quotient . . . . . . . . . . . . . . . . . . . 45

$5.2 \beta$-numbers and integral Menger curvature . . . . . . . . . . . . . . 48

$5.3 \beta$-numbers, approximating planes and angles . . . . . . . . . . . 59

6 Construction of the Lipschitz graph $\quad 63$

6.1 Partition of the support of the measure $\mu \ldots \ldots \ldots \ldots$. . . . . . 64

6.2 The distance to a well approximable ball . . . . . . . . . . . . . . . . . . 69

6.3 A Whitney-type decomposition of $P_{0} \backslash \pi(\mathcal{Z}) \ldots \ldots \ldots \ldots$. . . . . . . 74

6.4 Construction of the function $A \ldots \ldots \ldots \ldots \ldots$

$6.5 A$ is Lipschitz continuous . . . . . . . . . . . . . . . . . 79

7 -functions $\quad 87$

$7.1 \gamma$-functions and affine approximation of Lipschitz functions $\ldots \ldots \ldots$. . . . 90

7.2 The $\gamma$-function of $A$ and integral Menger curvature . . . . . . . . . . . 102

$8 \mathcal{Z}$ Is not too Small $\quad \mathbf{1 2 5}$

8.1 Most of $F$ is close to the graph of $A \ldots \ldots \ldots \ldots \ldots \ldots$

$8.2 \quad F_{1}$ is small . . . . . . . . . . . . . . . . . . . 139

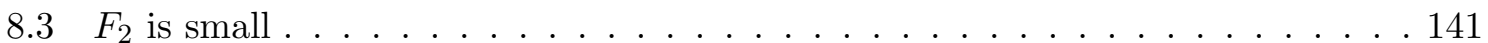

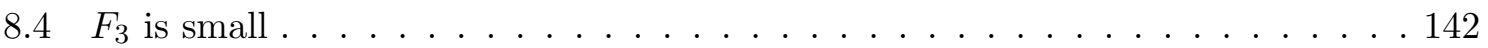


$\begin{array}{ll}\text { Appendix } & 149\end{array}$

A.1 Measuretheoretical statements . . . . . . . . . . . . . . . . . . . . . 149

A.2 Differentiation and Fourier transform on a linear subspace . . . . . . . . . . 156

A.3 Littlewood Paley theorem . . . . . . . . . . . . . . . . 160

$\begin{array}{ll}\text { Bibliography } & 165\end{array}$ 


\section{Introduction}

For three points $x, y, z \in \mathbb{R}^{N}$, we denote by $c(x, y, z)$ the inverse of the radius of the circumcircle determined by these three points. This expression is called Menger curvature ${ }^{1}$ of $x, y, z$. For a Borel set $E \subset \mathbb{R}^{N}$, we define by

$$
\mathcal{M}_{2}(E):=\int_{E} \int_{E} \int_{E} c^{2}(x, y, z) \mathrm{d} \mathcal{H}^{1}(x) \mathrm{d} \mathcal{H}^{1}(y) \mathrm{d} \mathcal{H}^{1}(z)
$$

the total Menger curvature of $E$, where $\mathcal{H}^{1}$ denotes the one-dimensional Hausdorff measure. In 1999, J.C. Léger proved the following theorem.

Theorem ([Lég99]). If $E \subset \mathbb{R}^{N}$ is some Borel set with $0<\mathcal{H}^{1}(E)<\infty$ and $\mathcal{M}_{2}(E)<\infty$, then $E$ is 1-rectifiable, i.e., there exists a countable family of Lipschitz functions $f_{i}: \mathbb{R} \rightarrow \mathbb{R}^{N}$ such that $\mathcal{H}^{1}\left(E \backslash \bigcup_{i} f_{i}(\mathbb{R})\right)=0$.

This result is an important step in the proof of Vitushkin's conjecture (for more details see [Tol14a, Dud10]), which states that a compact set with finite one-dimensional Hausdorff measure is removable for bounded analytic functions if and only if it is purely 1-unrectifiable, which means that every 1-rectifiable subset of this set has Hausdorff measure zero. A higher dimensional analogue of Vitushkin's conjecture is proven in [NTV14] but without using a higher dimensional version of Léger's theorem since in the higher dimensional setting there seems to be no connection between the $n$-dimensional Riesz transform and curvature (cf. introduction of [NTV14]).

There exist several generalisations of Léger's result. Hahlomaa proved in [Hah08, Hah07, Hah05] that if $X$ is a metric space and $\mathcal{M}_{2}(X)<\infty$, then $X$ is 1-rectifiable. Another version of this theorem dealing with sets of fractional Hausdorff dimension equal or less than $\frac{1}{2}$ is given by Lin and Mattila in [LM00].

In the present work, we generalise the result of Léger to arbitrary dimension and codimension, i.e., for $n$-dimensional subsets of $\mathbb{R}^{N}$ where $n \in \mathbb{N}$ satisfies $n<N$. In the case $n=N$ every $E \subset \mathbb{R}^{N}$ is $n$-rectifiable. On the one hand, it is quite clear which conclusion we want to obtain, namely that the set $E$ is $n$-rectifiable, which means that there exists a countable family of Lipschitz functions $f_{i}: \mathbb{R}^{n} \rightarrow \mathbb{R}^{N}$ such that $\mathcal{H}^{n}\left(E \backslash \bigcup_{i} f_{i}\left(\mathbb{R}^{n}\right)\right)=0$. On the other hand, it is by no means clear how to define integral Menger curvature for $n$-dimensional sets. Léger himself suggested an expression which turns out to be improper for our proof ${ }^{2}$ (cf. Lemma 3.12 in section 3.2). We characterise possible integrands for our result in Definition 3.1 , but for now let us start with an explicit example:

$$
\mathcal{K}\left(x_{0}, \ldots, x_{n+1}\right)=\frac{\mathcal{H}^{n+1}\left(\Delta\left(x_{0}, \ldots, x_{n+1}\right)\right)}{\Pi_{0 \leq i<j \leq n+1} d\left(x_{i}, x_{j}\right)},
$$

\footnotetext{
${ }^{1}$ Named after Karl Menger [Men30] who realized that $c(x, y, z)$ can be expressed purely in terms of mutual distances between the points. Menger's goal was a coordinate free metric geometry in contrast to classic differential geometry.

${ }^{2}$ Hence, we agree with a remark made by Lerman and Whitehouse at the end of the introduction of [LW09].
} 
where the numerator denotes the $(n+1)$-dimensional volume of the simplex spanned by the vertices $x_{0}, \ldots, x_{n+1}$, and $d\left(x_{i}, x_{j}\right)$ is the distance between $x_{i}$ and $x_{j}$. Using the law of sines, we obtain for $n=1$

$$
\mathcal{K}\left(x_{0}, x_{1}, x_{2}\right)=\frac{\mathcal{H}^{2}\left(\Delta\left(x_{0}, x_{1}, x_{2}\right)\right)}{d\left(x_{0}, x_{1}\right) d\left(x_{0}, x_{2}\right) d\left(x_{1}, x_{2}\right)}=\frac{1}{4} c\left(x_{0}, x_{1}, x_{2}\right) .
$$

Hence, $\mathcal{K}$ can be regarded as a generalisation of the original Menger curvature for higher dimensions. We set

$$
\mathcal{M}_{\mathcal{K}^{2}}(E):=\int_{E} \ldots \int_{E} \mathcal{K}^{2}\left(x_{0}, \ldots, x_{n+1}\right) \mathrm{d} \mathcal{H}^{n}\left(x_{0}\right) \ldots \mathrm{d} \mathcal{H}^{n}\left(x_{n+1}\right) .
$$

Now we can state our main theorem for this specific integrand (see Theorem 3.4 for the general version).

Theorem 1.1. If $E \subset \mathbb{R}^{N}$ is some Borel set with $\mathcal{M}_{\mathcal{K}^{2}}(E)<\infty$, then $E$ is n-rectifiable.

In the one-dimensional case, one gets 1-rectifiability for every connected set $E \subset \mathbb{R}^{N}$ with $\mathcal{H}^{1}(E)<\infty$ [Fed69, remark at the end of 3.3.22]. Federer mentions that no comparable result is valid for higher dimensional sets.

In the higher dimensional case, there exist well-known equivalent characterisations of $n$ rectifiability, for example, in terms of approximating tangent planes [Mat95, Thm. 15.19], orthogonal projections [Mat95, Thm. 18.1, Besicovitch-Federer projection theorem], and in terms of densities [Mat95, Thm. 17.6 and Thm. 17.8 (Preiss's theorem)]. Recently, Tolsa and Toro proved in [TT14] among other things that for some Borel set $E \subset \mathbb{R}^{N}$ with $\mathcal{H}^{n}(E)<\infty$, fulfilling some lower and upper density property, the condition

$$
\int_{0}^{1}\left|\frac{\mathcal{H}^{n}(B(x, r) \cap E)}{r^{n}}-\frac{\mathcal{H}^{n}(B(x, 2 r) \cap E)}{(2 r)^{n}}\right|^{2} \frac{\mathrm{d} r}{r}<\infty
$$

is equivalent to being $n$-rectifiable. For the case $n=1$, in [Tol14b], Tolsa could even get the same result without the density condition.

Now we present some of our own intermediate results that finally lead to the proof of Theorem 1.1, but that might also be of independent interest itself. A key tool are the so called $\beta$-numbers ${ }^{3}$ defined for $k>1, x \in \mathbb{R}^{N}, t>0, p \geq 1$ by

$$
\beta_{p ; k}(x, t):=\inf _{P \in \mathcal{P}(N, n)}\left(\frac{1}{t^{n}} \int_{B(x, k t)}\left(\frac{d(y, P)}{t}\right)^{p} \mathrm{~d} \mu(y)\right)^{\frac{1}{p}}
$$

where $\mathcal{P}(N, n)$ denotes the set of all $n$-dimensional planes in $\mathbb{R}^{N}, d(y, P)$ is the distance of $y$ to the $n$-dimensional plane $P$ and $\mu$ is a Borel measure on $\mathbb{R}^{N}$. There is a connection between those $\beta$-numbers and integral Menger curvature. In section 5.2, we prove the following theorem (see Theorem 5.6 for a more general version):

\footnotetext{
${ }^{3}$ Introduced by P. W. Jones in [Jon90] and [Jon91].
} 
Theorem 1.2. Let $\mu$ be some arbitrary Borel measure on $\mathbb{R}^{N}$ with compact support such that there is a constant $C \geq 1$ with $\mu(B) \leq C(\operatorname{diam} B)^{n}$ for all balls $B \subset \mathbb{R}^{N}$ (one may call this upper Ahlfors regularity), where diam $B$ denotes the diameter of the ball $B$. Let $B(x, t)$ be a fixed ball with $\mu(B(x, t)) \geq \lambda t^{n}$ for some $\lambda>0$ and let $k>2$. Then there exist some constants $k_{1}>1$ and $C \geq 1$ such that

$$
\beta_{2 ; k}(x, t)^{2} \leq \frac{C}{t^{n}} \int_{B\left(x, k_{1} t\right)} \ldots \int_{B\left(x, k_{1} t\right)} \chi_{D}\left(x_{0}, \ldots, x_{n}\right) \mathcal{K}^{2}\left(x_{0}, \ldots, x_{n+1}\right) \mathrm{d} \mu\left(x_{0}\right) \ldots \mathrm{d} \mu\left(x_{n+1}\right),
$$

where $D=\left\{\left(x_{0}, \ldots, x_{n+1}\right) \in B\left(x, k_{1} t\right)^{n+2} \mid d\left(x_{i}, x_{j}\right) \geq \frac{t}{k_{1}}, i \neq j\right\}$.

A measure $\mu$ is said to be $n$-dimensional Ahlfors regular if and only if there exists some constant $C \geq 1$ so that $\frac{1}{C}(\operatorname{diam} B)^{n} \leq \mu(B) \leq C(\operatorname{diam} B)^{n}$ for all balls $B$ with centre on the support of $\mu$. We mention that we do not have to assume for this theorem that the measure $\mu$ is $n$-dimensional Ahlfors regular. We only need the upper Ahlfors regularity and the condition $\mu(B(x, t)) \geq \lambda t^{n}$ for this specific ball $B(x, t)$.

Lerman and Whitehouse obtain a comparable result in [LW09, Thm. 1.1]. The main differences are that, on the one hand, they have to use an $n$-dimensional Ahlfors regular measure, but, on the other hand, they work in a real separable Hilbert space of possibly infinite dimension instead of $\mathbb{R}^{N}$. The higher dimensional Menger curvatures they used (see [LW09, introduction and section 6]) are examples of integrands that also fit in our more general setting $^{4}$. This means that all of our results are valid if one uses their integrands instead of the initial $\mathcal{K}$ presented as an example above.

In addition to rectifiability, there is the notion of uniform rectifiability, which implies rectifiability. A set is uniformly rectifiable if it is Ahlfors regular ${ }^{5}$ and if it fulfils a second condition in terms of $\beta$-numbers (cf. [DS93, Thm. 1.57, (1.59)]). In [LW09] and [LW11], Lerman and Whitehouse give an alternative characterisation of uniform rectifiability by proving that for an Ahlfors regular set this $\beta$-number term is comparable to a term expressed with integral Menger curvature. One of the two inequalities needed is given in [LW09, Thm. 1.3], and is similar to our following theorem, which is a consequence of Theorem 1.2 in connection with Fubini's theorem (see Theorem 5.7 for a more general version). We emphasise again that in our case the measure $\mu$ does not have to be Ahlfors regular.

Theorem 1.3. Let $\mu, \lambda$ and $k$ be as in the previous theorem. There exists a constant $C \geq 1$ such that

$$
\iint_{0}^{\infty} \beta_{2 ; k}(x, t)^{2} \mathbb{1}_{\left\{\mu(B(x, t)) \geq \lambda t^{n}\right\}} \frac{\mathrm{d} t}{t} \mathrm{~d} \mu(x) \leq C \mathcal{M}_{\mathcal{K}^{2}}(\mu) .
$$

In the last years, there occurred several papers working with integral Menger curvatures. Some deal with (one-dimensional) space curves and get higher regularity $\left(C^{1, \alpha}\right)$ of the arc length parametrisation if the integral Menger curvature is finite, e.g [SSvdM09, SSvdM10]. Others handle higher dimensional objects in [Kol12, KSvdM13, SvdM11] occasionally using versions of integral Menger curvatures similar to ours ${ }^{6}$. Remarkable are the results of Blatt

\footnotetext{
${ }^{4} \mathrm{~A}$ characterisation of all possible integrands for our result can be found at the beginning of section 3.1. In Lemma 3.8, we discuss one of the integrands of Lerman and Whitehouse.

${ }^{5} \mathrm{~A}$ set $E$ is $n$-dimensional Ahlfors regular if and only if the restricted Hausdorff measure $\mathcal{H}^{n} \mathrm{~L} E$ is $n$ dimensional Ahlfors regular.

${ }^{6}$ Our main result does not work with their integrands, but most of the partial results are valid, cf. section 3.2.
} 
and Kolasinski [BK12, Bla13]. They proved among other things that for $p>n(n+1)$ and some compact $n$-dimensional $C^{1}$ manifold $\Sigma$

$$
\mathcal{E}_{p}^{n+1}=\int_{\Sigma} \ldots \int_{\Sigma}\left(\frac{\mathcal{H}^{n+1}\left(\Delta\left(x_{0}, \ldots, x_{n+1}\right)\right)}{\operatorname{diam}\left(\Delta\left(x_{0}, \ldots, x_{n+1}\right)\right)^{n+2}}\right)^{p} \mathrm{~d} \mathcal{H}^{n}\left(x_{0}\right) \ldots, \mathrm{d} \mathcal{H}^{n}\left(x_{n+1}\right)<\infty
$$

is equivalent to having a local representation as the graph of a function belonging to the Sobolev Slobodeckij space $W^{2-\frac{n(n+1)}{p}, p}$.

Finally, we mention that in [SSvdM13, SvdM13a] Menger curvature energies are recently used as knot energies in geometric knot theory to avoid some of the drawbacks of self-repulsing potentials by the Möbius energy [O'H91, FHW94].

\section{Sketch of the proof}

Theorem 1.1 is proven by an indirect argumentation following the general strategy of Léger. We assume that the set $E$ is not $n$-rectifiable and a subset of $B(0,1)$. It is possible (cf. Lemma A.1) to decompose $E$ into two disjoint subsets $E_{r}$ and $E_{u}$ where one is $n$-rectifiable and the other one is purely $n$-unrectifiable. Herein, a set $E_{u}$ is purely $n$-unrectifiable if we have $\mathcal{H}^{n}\left(E_{u} \cap f\left(\mathbb{R}^{n}\right)\right)=0$ for every Lipschitz function $f: \mathbb{R}^{n} \rightarrow \mathbb{R}^{N}$. Our assumption implies that $\mathcal{H}^{n}\left(E_{u}\right)>0$. It is possible to find a subset $\hat{E}_{u}$ of $E_{u}$ with $\mathcal{H}^{n}\left(\hat{E}_{u}\right)>0$ and $\hat{E}_{u}$ is compact, upper Ahlfors regular and has very small integral Menger curvature. Since $\hat{E}_{u}$ is a subset of $E$, we obtain $\mathcal{M}_{\mathcal{K}^{2}}\left(\hat{E}_{u}\right)<\infty$. To get a contradiction it is now enough to define a Lipschitz function $f: \mathbb{R}^{n} \rightarrow \mathbb{R}^{N}$ with $\mathcal{H}^{n}\left(\hat{E}_{u} \cap f\left(\mathbb{R}^{n}\right)\right)>0$. In order to define this function $f$, we will define some function $A: P_{0} \rightarrow P_{0}^{\perp}$ in chapter 6 , where $P_{0} \subset \mathbb{R}^{N}$ is an $n$-dimensional subspace of $\mathbb{R}^{N}$ and $P_{0}^{\perp}$ denotes the orthogonal complement of $P_{0}$. We choose $P_{0}$ such that it is a good approximation of $\hat{E}_{u}$ in the sense of $\beta$-numbers. This is possible since the $\beta$-numbers are controlled by the integral Menger curvature and we choose $\hat{E}_{u}$ keeping the integral Menger curvature of $\hat{E}_{u}$ small.

Next, we divide $\hat{E}_{u}$ in four parts, namely $\mathcal{Z}$ and $F_{1}, F_{2}, F_{3} . F_{1}$ will be the set which collects the thin parts of $\hat{E}_{u}$ and in $F_{2}$ we collect those parts of $\hat{E}_{u}$ with locally large $\beta$-numbers. The construction of $F_{3}$ is a bit more complicated. We combine all components of $\hat{E}_{u}$, where in a local sense good approximations by $n$-planes exist, but all those planes have a large angle to the plane $P_{0}$. Finally, the rest of $\hat{E}_{u}$ is in $\mathcal{Z}$.

Now it is possible to define a Lipschitz continuous function $A: \mathbb{R}^{n} \rightarrow \mathbb{R}^{N}$ such that the graph of $A$ covers $\mathcal{Z}$. All this is done in chapter 6 .

The following chapters 7 and 8 prove that the set $\mathcal{Z}$ can not be a null set which implies that we have covered a non-trivial part of $\hat{E}_{u}$ with the graph of $A$. This is in contradiction to the assumption that $E_{u} \supset \hat{E}_{u}$ is purely $n$-unrectifialble. It is relatively easy to see that the thin set $F_{1}$ is small. The set $F_{2}$ is small since, with the Theorem 1.3, we have controlled the size of all $\beta$-numbers from small to large scale by the integral Menger curvature. It is more complicated to prove that $F_{3}$ is small. For this purpose, we introduce the expression

$$
\gamma_{A}(q, t):=\inf _{\substack{a \\ \text { affine function }}} \frac{1}{t^{n}} \int_{B(q, t) \cap P_{0}} \frac{d(A(u), a(u))}{t} \mathrm{~d} \mathcal{H}^{n}(u)
$$

and get some control on this expression by the integral Menger curvature, similar to the one 
we get on the $\beta$-numbers (cf. section 7.2). Due to the fact that the function $A$ was constructed approximating $F_{3}$, the control we have on the $\gamma$-functions finally implies that even $F_{3}$ is small.

We mention that exactly this final step, the smallness of $F_{3}$, is the one which reduces the amount of possible integrands for the main result (cf. Definition 3.1) to those that have the right scaling behaviour together with the integrability exponent $p=2$. For example, the integrand used in [KSvdM13]

$$
\frac{\mathcal{H}^{n+1}\left(\Delta\left(x_{0}, \ldots, x_{n+1}\right)\right)}{\operatorname{diam} \Delta\left(x_{0}, \ldots, x_{n+1}\right)^{n+2}}
$$

together with the integrability exponent $p=n(n+1)$ is suitable for all parts of our proof except for the last one. If you change the exponent of the denominator from $n+2$ to $\frac{(n+1)(n+2)}{2}$ together with integrability exponent $p=2$, the main result is valid again.

\section{Organisation of this work}

In chapter 2 , we introduce simplices and give some notation and basic results. Furthermore, we define the angle between affine subspaces and state some lemmas working with this notion. In the next chapter, we can give the precise formulation of our main result Theorem 3.4 including the characterisation of integrands and integral Menger curvatures for which our main result holds. In particular, Theorem 1.1 turns out to be a simple corollary of this main result. After that, we give some examples of integrands known from several papers working with integral Menger curvatures and explain which of those are suitable for our setting. The following chapters 4 to 8 give the proof of our main result following the sketch given above. In chapter 5 , we present some results for a Borel measure including the general versions of Theorems 1.2 and 1.3, namely Theorem 5.6 and 5.7. We remark that all statements in chapter 6,7 and 8 , except section 7.1, depend on the construction given in chapter 6 . 



\section{Preliminaries}

\subsection{Basic notation}

Let $n, m, N \in \mathbb{N}$ with $1 \leq n<N$ and $1 \leq m<N$. If $E \subset \mathbb{R}^{N}$ is some subset of $\mathbb{R}^{N}$, we write $\bar{E}$ for its closure and $\stackrel{\circ}{E}$ for its interior. We set $d(x, y):=|x-y|$ where $x, y \in \mathbb{R}^{N}$ and $|\cdot|$ is the usual Euclidean norm. Furthermore, for $x \in \mathbb{R}^{N}$ and $E_{1}, E_{2} \subset \mathbb{R}^{N}$, we set $d\left(x, E_{2}\right)=\inf _{y \in E_{2}} d(x, y), d\left(E_{1}, E_{2}\right)=\inf _{z \in E_{1}} d\left(z, E_{2}\right)$ and \#E means the number of elements of $E$. By $B(x, r)$ we denote the closed ball in $\mathbb{R}^{N}$ with centre $x$ and radius $r$, and we define by $\omega_{n}$ the $n$-dimensional volume of the $n$-dimensional unit ball.

\subsection{Basic linear algebra facts}

Let $G(N, m)$ be the Grassmannian, the space of all $m$-dimensional linear subspaces of $\mathbb{R}^{N}$ and $\mathcal{P}(N, m)$ the set of all $m$-dimensional affine subspaces of $\mathbb{R}^{N}$. For $P \in \mathcal{P}(N, m)$, we define $\pi_{P}$ as the orthogonal projection on $P$, i.e., for a point $a \in \mathbb{R}^{n}$ the point $\pi_{P}(a)$ is the unique point in $P$ that fulfils $\left[\pi_{P}(a)-a\right] \perp P$. If $P \in \mathcal{P}(N, m)$, we have that $P-\pi_{P}(0) \in G(N, m)$, hence $P-\pi_{P}(0)$ is the linear subspace parallel to $P$. Furthermore, we set $\pi_{P}^{\perp}:=\pi_{P-\pi_{P}(0)}^{\perp}:=$ $\pi_{\left(P-\pi_{P}(0)\right)^{\perp}}$ where $\pi_{\left(P-\pi_{P}(0)\right)^{\perp}}$ is the orthogonal projection on the orthogonal complement of $P-\pi_{P}(0)$. This implies that $\pi_{P}^{\perp}=\pi_{\tilde{P}}$ and $\pi_{P} \neq \pi_{\tilde{P}}$ whenever $P$ is parallel but not equal to $\tilde{P}$.

Furthermore, for $A \subset \mathbb{R}^{N}$ and $x \in \mathbb{R}^{N}$, we set $A+x:=\left\{y \in \mathbb{R}^{n} \mid y-x \in A\right\}$. By $\operatorname{span}(A)$, we denote the linear subspace of $\mathbb{R}^{N}$ spanned by the elements of $A$. If $A=\left\{o_{1}, \ldots, o_{m}\right\}$ or $A=A_{1} \cup A_{2}$, we may write $\operatorname{span}\left(o_{1}, \ldots, o_{m}\right)$ resp. $\operatorname{span}\left(A_{1}, A_{2}\right)$ instead of $\operatorname{span}(A)$.

Lemma 2.1. Let $P \in \mathcal{P}(N, m)$ and $a, x \in \mathbb{R}^{N}$. We have

$$
\pi_{P}(a)=\pi_{P-x}(a-x)+x .
$$

Proof. The point $\pi_{P-x}(a-x)$ is in $P-x$ and so $\pi_{P-x}(a-x)+x$ is in $P$. Furthermore $\pi_{P-x}(a-x)$ fulfils $\left[\pi_{P-x}(a-x)-(a-x)\right] \perp P-x$ where $P-x$ is parallel to $P$. So we conclude that $\left[\left(\pi_{P-x}(a-x)+x\right)-a\right] \perp P$ which implies that $\pi_{P-x}(a-x)+x=\pi_{P}(a)$.

Lemma 2.2. Let $b, a, a_{i} \in \mathbb{R}^{N}, \alpha_{i} \in \mathbb{R}$ for $i=1, . . l, l \in \mathbb{N}$ with

$$
b=a+\sum_{i=1}^{l} \alpha_{i}\left(a_{i}-a\right)
$$

and $P \in \mathcal{P}(N, m)$. Then we have

$$
\pi_{P}(b)=\pi_{P}(a)+\sum_{i=1}^{l} \alpha_{i}\left[\pi_{P}\left(a_{i}\right)-\pi_{P}(a)\right] .
$$


For those $b \in \mathbb{R}^{N}$, the projection $\pi_{P}$ seems to be linear, although $P$ is an affine subspace.

Proof. We choose $x \in P$ so that $P-x$ is a linear subspace of $\mathbb{R}^{N}$. Using Lemma 2.1, we get

$$
\begin{aligned}
\pi_{P}\left(a+\sum_{i=1}^{l} \alpha_{i}\left(a_{i}-a\right)\right) & =\pi_{P-x}\left(a+\sum_{i=1}^{l} \alpha_{i}\left(a_{i}-a\right)-x\right)+x \\
& =\pi_{P-x}(a-x)+x+\sum_{i=1}^{l} \alpha_{i}\left[\pi_{P-x}\left(a_{i}-x\right)+x-\pi_{P-x}(a-x)-x\right] \\
& =\pi_{P}(a)+\sum_{i=1}^{l} \alpha_{i}\left[\pi_{P}\left(a_{i}\right)-\pi_{P}(a)\right] .
\end{aligned}
$$

Lemma 2.3. With the requirements of Lemma 2.2, we have

$$
d(b, P) \leq d(a, P)+\sum_{i=1}^{l}\left|\alpha_{i}\right|\left(d\left(a_{i}, P\right)+d(a, P)\right) .
$$

Proof. With Lemma 2.2, we get

$$
\begin{aligned}
d(b, P) & =\left|b-\pi_{P}(b)\right| \\
& =\left|a+\sum_{i=1}^{l} \alpha_{i}\left(a_{i}-a\right)-\left(\pi_{P}(a)+\sum_{i=1}^{l} \alpha_{i}\left[\pi_{P}\left(a_{i}\right)-\pi_{P}(a)\right]\right)\right| \\
& \leq d(a, P)+\sum_{i=1}^{l}\left|\alpha_{i}\right|\left(d\left(a_{i}, P\right)+d(a, P)\right) .
\end{aligned}
$$

Lemma 2.4. Let $U, V \in G(N, m)$ with $\operatorname{dim} U=\operatorname{dim} V=m<N$ and $\operatorname{dim}(U \cap V)=m-1$. For $u_{1}, u_{2} \in U \backslash V$, we have

$$
\frac{\left|u_{1}-\pi_{V}\left(u_{1}\right)\right|}{\left|u_{1}-\pi_{U \cap V}\left(u_{1}\right)\right|}=\frac{\left|u_{2}-\pi_{V}\left(u_{2}\right)\right|}{\left|u_{2}-\pi_{U \cap V}\left(u_{2}\right)\right|} .
$$

Proof. We choose an orthonormal basis of $U \cap V=\operatorname{span}\left(o_{1}, \ldots, o_{m-1}\right)$ and extend this to orthonormal bases of $U$ and of $V$ :

$$
\begin{aligned}
& U=\operatorname{span}\left(o_{1}, \ldots, o_{m-1}, o_{U}\right), \\
& V=\operatorname{span}\left(o_{1}, \ldots, o_{m-1}, o_{V}\right) .
\end{aligned}
$$

For $u_{1}, u_{2} \in U \backslash V$, we find $\alpha_{i}, \alpha_{u_{1}} \beta_{i}, \beta_{u_{2}} \in \mathbb{R}, i=1, \ldots, m-1$, with

$$
\begin{aligned}
& u_{1}=\sum_{i=1}^{m-1} \alpha_{i} o_{i}+\alpha_{u_{1}} o_{U}, \\
& u_{2}=\sum_{i=1}^{m-1} \beta_{i} o_{i}+\beta_{u_{2}} o_{U} .
\end{aligned}
$$


With $\pi_{V}\left(o_{i}\right)=o_{i}$ for $i \in\{1, \ldots, m-1\}$, we obtain

$$
\pi_{V}\left(u_{1}\right)=\sum_{i=1}^{m-1} \alpha_{i} o_{i}+\alpha_{u_{1}} \pi_{V}\left(o_{U}\right)
$$

and consequently

$$
u_{1}-\pi_{V}\left(u_{1}\right)=\alpha_{u_{1}}\left(o_{U}-\pi_{V}\left(o_{U}\right)\right) .
$$

Furthermore, we deduce

$$
u_{1}-\pi_{U \cap V}\left(u_{1}\right)=\alpha_{u_{1}} o_{U}-\underbrace{\pi_{U \cap V}\left(\alpha_{u_{1}} o_{U}\right)}_{=0}=\alpha_{u_{1}} o_{U} .
$$

For $u_{2}$ we get corresponding equations and so we conclude

$$
\begin{aligned}
\frac{\left|u_{1}-\pi_{V}\left(u_{1}\right)\right|}{\left|u_{1}-\pi_{U \cap V}\left(u_{1}\right)\right|} & =\frac{\left|\alpha_{u_{1}}\left(o_{U}-\pi_{V}\left(o_{U}\right)\right)\right|}{\left|\alpha_{u_{1}} o_{U}\right|} \\
& =\left|o_{U}-\pi_{V}\left(o_{U}\right)\right| \\
& =\frac{\left|u_{2}-\pi_{V}\left(u_{2}\right)\right|}{\left|u_{2}-\pi_{U \cap V}\left(u_{2}\right)\right|} .
\end{aligned}
$$

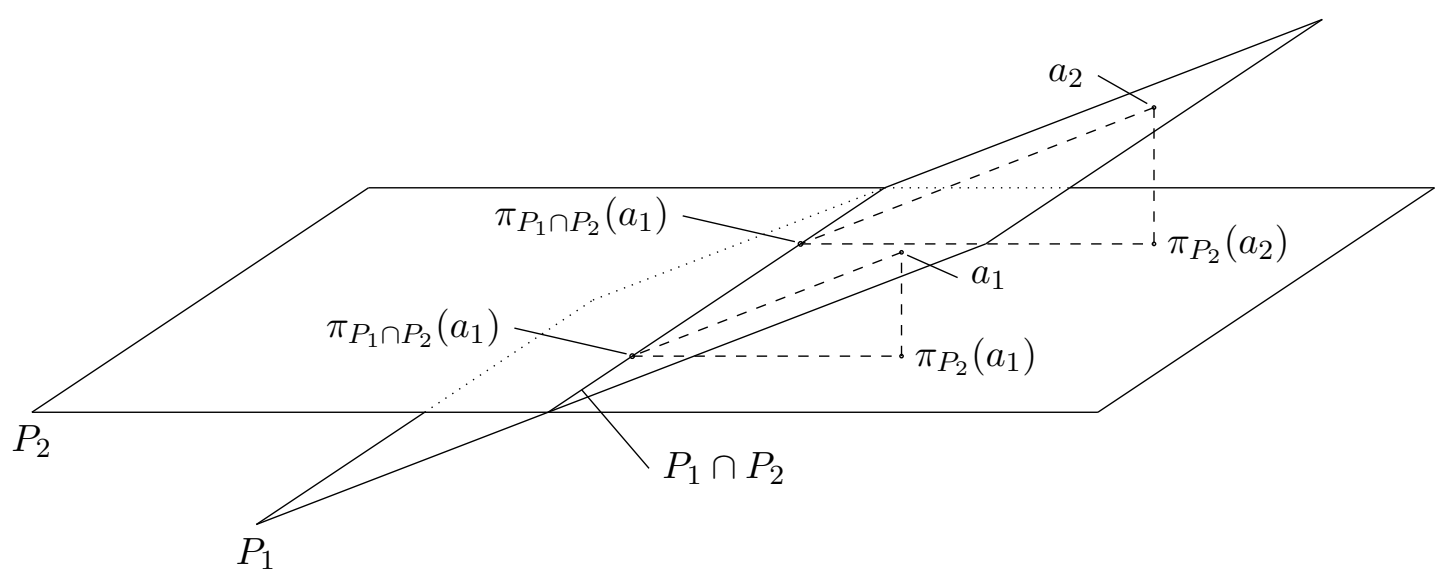

Figure 2.1: Illustration of Corollary 2.5: $\frac{\left|a_{1}-\pi_{P_{2}}\left(a_{1}\right)\right|}{\left|a_{1}-\pi_{P_{1} \cap P_{2}}\left(a_{1}\right)\right|}=\frac{\left|a_{2}-\pi_{P_{2}}\left(a_{2}\right)\right|}{\left|a_{2}-\pi_{P_{1} \cap P_{2}}\left(a_{2}\right)\right|}$

Corollary 2.5. Let $P_{1}, P_{2} \in \mathcal{P}(N, m)$ with $\operatorname{dim} P_{1}=\operatorname{dim} P_{2}=m<N$ and $\operatorname{dim}\left(P_{1} \cap P_{2}\right)=$ $m-1$. For $a_{1}, a_{2} \in P_{1} \backslash P_{2}$, we have

$$
\frac{\left|a_{1}-\pi_{P_{2}}\left(a_{1}\right)\right|}{\left|a_{1}-\pi_{P_{1} \cap P_{2}}\left(a_{1}\right)\right|}=\frac{\left|a_{2}-\pi_{P_{2}}\left(a_{2}\right)\right|}{\left|a_{2}-\pi_{P_{1} \cap P_{2}}\left(a_{2}\right)\right|} .
$$


Proof. Choose some $x \in P_{1} \cap P_{2}$, set $U:=P_{1}-x, V:=P_{2}-x, u_{1}:=a_{1}-x, u_{2}:=a_{2}-x$ and use Lemma 2.4 as well as

$$
\left|a_{i}-\pi_{P_{2}}\left(a_{i}\right)\right|=\left|a_{i}-x-\left(\pi_{P_{2}}\left(a_{i}\right)-x\right)\right|=\left|a_{i}-x-\pi_{P_{2}-x}\left(a_{i}-x\right)\right|=\left|u_{i}-\pi_{V}\left(u_{i}\right)\right|
$$

and

$$
\left|a_{i}-\pi_{P_{1} \cap P_{2}}\left(a_{i}\right)\right|=\left|a_{i}-x-\pi_{\left(P_{1}-x\right) \cap\left(P_{2}-x\right)}\left(a_{i}-x\right)\right|=\left|u_{i}-\pi_{U \cap V}\left(u_{i}\right)\right|
$$

where we have used Lemma 2.1 and $i \in\{1,2\}$.

Lemma 2.6. Let $A, B$ be affine subspaces of $\mathbb{R}^{N}$ with $A \subset B$ and let $a \in \mathbb{R}^{N}$. We have

$$
\pi_{A}\left(\pi_{B}(a)\right)=\pi_{A}(a)=\pi_{B}\left(\pi_{A}(a)\right) .
$$

Proof. Due to $A \subset B$, the second equality is obvious, so we focus on the first one. Let $x \in A \subset B$. We deduce that $A-x, B-x$ are linear subspaces of $\mathbb{R}^{N}$. Let $\left(o_{1}, \ldots, o_{l}\right)$ be an orthonormal basis of $A-x,\left(o_{1}, \ldots, o_{l}, o_{l+1}, \ldots, o_{m}\right)$ be an orthonormal basis of $B-x$ and $\left(o_{1}, \ldots, o_{N}\right)$ be an orthonormal basis of $\mathbb{R}^{N}$. There exist some $\alpha_{i} \in \mathbb{R}$ with

$$
a-x=\sum_{i=1}^{N} \alpha_{i} o_{i}
$$

So we get

$$
\pi_{A-x}\left(\pi_{B-x}(a-x)\right)=\pi_{A-x}\left(\sum_{i=1}^{m} \alpha_{i} o_{i}\right)=\sum_{i=1}^{l} \alpha_{i} o_{i}=\pi_{A-x}(a-x) .
$$

We conclude with Lemma 2.1

$$
\pi_{A}\left(\pi_{B}(a)\right)-x=\pi_{A-x}\left(\pi_{B-x}(a-x)\right)=\pi_{A-x}(a-x)=\pi_{A}(a)-x .
$$

\subsection{Simplices}

Definition 2.7. Let $x_{i} \in \mathbb{R}^{N}$ for $i=0,1, \ldots, m$. We define $\Delta\left(x_{0}, \ldots, x_{m}\right)=\Delta\left(\left\{x_{0}, \ldots, x_{m}\right\}\right)$ as the convex hull of the set $\left\{x_{0}, \ldots, x_{m}\right\}$ and call it simplex or $m$-simplex when $m=$ $\operatorname{dim}_{\mathcal{H}}\left(\Delta\left(x_{0}, \ldots, x_{m}\right)\right)$, the Hausdorff dimension of $\Delta\left(x_{0}, \ldots, x_{m}\right)$.

Notation. If the vertices of $T=\Delta\left(x_{0}, \ldots, x_{m}\right)$ are in some set $G \subset \mathbb{R}^{N}$, i.e., $x_{0}, \ldots, x_{m} \in G$, we write $T=\Delta\left(x_{0}, \ldots, x_{m}\right) \in G$.

With aff $(E)$ we denote the smallest affine subspace of $\mathbb{R}^{N}$ that contains the set $E \subset \mathbb{R}^{N}$. If $E=\left\{x_{0}\right\}$, we set $\operatorname{aff}(E)=\left\{x_{0}\right\}$.

Definition 2.8. Let $T=\Delta\left(x_{0}, \ldots, x_{m}\right) \in \mathbb{R}^{N}$. For $i, j \in\{0,1, \ldots, m\}$ we set

$$
\begin{aligned}
\mathfrak{f c}_{i} T=\mathfrak{f c}_{x_{i}} T & =\Delta\left(\left\{x_{0}, \ldots, x_{m}\right\} \backslash\left\{x_{i}\right\}\right), \\
\mathfrak{f c}_{i, j} T=\mathfrak{f c}_{x_{i}, x_{j}} T & =\Delta\left(\left\{x_{0}, \ldots, x_{m}\right\} \backslash\left\{x_{i}, x_{j}\right\}\right), \\
\mathfrak{h}_{i} T=\mathfrak{h}_{x_{i}} T & =d\left(x_{i}, \operatorname{aff}\left(\left\{x_{0}, \ldots, x_{m}\right\} \backslash\left\{x_{i}\right\}\right)\right) .
\end{aligned}
$$


Definition 2.9. Let $T=\Delta\left(x_{0}, \ldots, x_{m}\right)$ be an $m$-simplex in $\mathbb{R}^{N}$. If $\mathfrak{h}_{i} T \geq \sigma$ for all $i=$ $0,1, \ldots, m$, we call $T$ an $(m, \sigma)$-simplex.

Remark 2.10. Let $T=\Delta\left(x_{0}, \ldots, x_{m}\right)$ an $(m, \sigma)$-simplex. For all $i \in\{0, \ldots, m\}$, we have

$$
d\left(x_{i}, \operatorname{aff}\left(A_{i}\right)\right) \geq \mathfrak{h}_{i} T \geq \sigma
$$

for every $\emptyset \neq A_{i} \subset\left\{x_{0}, \ldots, x_{m}\right\} \backslash\left\{x_{i}\right\}$.

Definition 2.11. Let $T=\Delta\left(x_{0}, \ldots, x_{m}\right)$ be an $m$-simplex in $\mathbb{R}^{N}$. By $\mathcal{H}^{m}(T)$ we denote the volume of $T$ and we define the normalized volume

$$
\mathfrak{v}(T):=m ! \mathcal{H}^{m}(T)
$$

which is the volume of the parallelotope spanned by the simplex $T$ (cf. [Ste66]). We also have a characterisation of $\mathfrak{v}(T)$ by the Gram determinant

$$
\mathfrak{v}(T)=\sqrt{\operatorname{Gram}\left(x_{1}-x_{0}, \ldots, x_{m}-x_{0}\right)},
$$

where the Gram determinant of vectors $v_{1}, \ldots, v_{m} \in \mathbb{R}^{N}$ is defined by

$$
\operatorname{Gram}\left(v_{1}, \ldots, v_{m}\right):=\operatorname{det}\left(\left(v_{1}, \ldots, v_{m}\right)^{T}\left(v_{1}, \ldots, v_{m}\right)\right) .
$$

Remark 2.12. Let $T=\Delta\left(x_{0}, \ldots, x_{m}\right)$ be an $m$-simplex. The volume of the parallelotope, spanned by $T$, fulfils

$$
\mathfrak{v}(T)=\mathfrak{h}_{i} T \mathfrak{v}\left(\mathfrak{f}_{i} T\right)
$$

which implies

$$
\mathcal{H}^{m}(T)=\frac{1}{m} \mathfrak{h}_{i} T \mathcal{H}^{m-1}\left(\mathfrak{f}_{i} T\right)
$$

for the volume of a simplex.

Lemma 2.13. Let $T=\Delta\left(x_{0}, \ldots, x_{m}\right)$ be an $m$-simplex. We have

$$
\frac{\mathfrak{h}_{i} T}{\mathfrak{h}_{i} \mathfrak{f}_{j} T}=\frac{\mathfrak{h}_{j} T}{\mathfrak{h}_{j} \mathfrak{c}_{i} T}
$$

Proof. We have

$$
\begin{aligned}
\frac{\mathfrak{h}_{i}(T)}{\mathfrak{h}_{i}\left(\mathfrak{f}_{j} T\right)} & =\frac{\mathfrak{v}(T)}{\mathfrak{h}_{i}\left(\mathfrak{f c}_{j} T\right) \mathfrak{v}\left(\mathfrak{f}_{i} T\right)} \\
& =\frac{\mathfrak{h}_{j}(T) \mathfrak{v}\left(\mathfrak{f} \mathfrak{c}_{j} T\right)}{\mathfrak{h}_{i}\left(\mathfrak{f}_{j} T\right) \mathfrak{h}_{j}\left(\mathfrak{f}_{i} T\right) \mathfrak{v}\left(\mathfrak{f}_{i, j} T\right)} \\
& =\frac{\mathfrak{h}_{j}(T) \mathfrak{v}\left(\mathfrak{f}_{j} T\right)}{\mathfrak{h}_{j}\left(\mathfrak{f}_{i} T\right) \mathfrak{v}\left(\mathfrak{f}_{j} T\right)} \\
& =\frac{\mathfrak{h}_{j}(T)}{\mathfrak{h}_{j}\left(\mathfrak{f}_{i} T\right)}
\end{aligned}
$$


Lemma 2.14. Let $0<h<H, 1 \leq m \leq N+1$ and $y_{0}, x_{i} \in \mathbb{R}^{N}, i=0,1, \ldots, m$. If $T_{x}=\Delta\left(x_{0}, \ldots, x_{m}\right)$ is an $(m, H)$-simplex and $d\left(y_{0}, x_{0}\right) \leq h$, then $T_{y}=\Delta\left(y_{0}, x_{1}, \ldots, x_{m}\right)$ is an $(m, H-h)$-simplex.

Proof. We have

$$
\mathfrak{h}_{0} T_{y} \geq \mathfrak{h}_{0} T_{x}-d\left(x_{0}, y_{0}\right) \geq H-h .
$$

Now, we show that $\mathfrak{h}_{1} T_{y} \geq H-h$.

If $m=1$, we have $\mathfrak{h}_{1} T_{y}=d\left(y_{0}, x_{1}\right)=\mathfrak{h}_{0} T_{y}$. So we can assume that $m \geq 2$ for the rest of this proof. We set $z_{0}:=\pi_{\mathrm{aff}_{\left(\mathfrak{f}_{1} T_{y}\right)}}\left(x_{0}\right)$,

$$
T_{z}:=\Delta\left(z_{0}, x_{1}, \ldots, x_{m}\right)
$$

and start with some intermediate results:

I. Due to $\mathfrak{h}_{0} T_{y} \geq H-h>0, T_{y}$ is an $m$-simplex.

II. We get

$$
d\left(x_{0}, z_{0}\right)=d\left(x_{0}, \operatorname{aff}\left(\mathfrak{f c}_{1} T_{y}\right)\right) \leq d\left(x_{0}, y_{0}\right) \leq h .
$$

III. Due to $z_{0} \in \operatorname{aff}\left(\mathfrak{f c}_{1} T_{y}\right)$, there exists some $r_{i} \in \mathbb{R}, i=0,3, \ldots, m$ with

$$
z_{0}=x_{2}+r_{0}\left(y_{0}-x_{2}\right)+\sum_{j=3}^{m} r_{j}\left(x_{j}-x_{2}\right) .
$$

IV. We get with Lemma 2.2 and because of $\pi_{\mathrm{aff}\left(\mathfrak{f}_{0} T_{x}\right)}\left(x_{i}\right)=x_{i}$ for $i=2, \ldots m$

$$
\begin{aligned}
\mathfrak{h}_{0} T_{z}=d\left(z_{0}, \operatorname{aff}\left(\mathfrak{f c}_{0} T_{x}\right)\right) & =\left|z_{0}-\pi_{\operatorname{aff}\left(\mathfrak{f}_{0} T_{x}\right)}\left(z_{0}\right)\right| \\
& =\left|r_{0} y_{0}-r_{0} \pi_{\text {aff }\left(\mathfrak{f}_{0} T_{x}\right)}\left(y_{0}\right)\right| \\
& =r_{0} d\left(y_{0}, \operatorname{aff}\left(\mathfrak{f c}_{0} T_{x}\right)\right) \\
& =r_{0} \mathfrak{h}_{0}\left(T_{y}\right) .
\end{aligned}
$$

By using $\pi_{\mathrm{aff}\left(\mathfrak{f}_{0,1} T_{x}\right)}\left(x_{i}\right)=x_{i}$ for $i=2, \ldots m$, we get analogously

$$
\begin{aligned}
\mathfrak{h}_{0}\left(\mathfrak{f c}_{1} T_{z}\right)=d\left(z_{0}, \operatorname{aff}\left(\mathfrak{f c}_{0,1} T_{x}\right)\right) & =\left|z_{0}-\pi_{\operatorname{aff}\left(\mathfrak{f c}_{0,1} T_{x}\right)}\left(z_{0}\right)\right| \\
& =\left|r_{0} y_{0}-r_{0} \pi_{\mathrm{aff}\left(\mathfrak{f}_{0,1} T_{x}\right)}\left(y_{0}\right)\right| \\
& =r_{0} d\left(y_{0}, \operatorname{aff}\left(\mathfrak{f c}_{0,1} T_{x}\right)\right) \\
& =r_{0} \mathfrak{h}_{0}\left(\mathfrak{f}_{1} T_{y}\right) .
\end{aligned}
$$

V. The plane $\operatorname{aff}\left(\mathfrak{f c}_{1} T_{y}\right)$ is an affine subspace of $\operatorname{aff}\left(\mathfrak{f c}_{0,1} T_{x}\right)$, so with Lemma 2.6 , we get 
$\pi_{\mathrm{aff}\left(\mathfrak{f}_{0,1} T_{x}\right)}\left(z_{0}\right)=\pi_{\mathrm{aff}\left(\mathfrak{f}_{0,1} T_{x}\right)}\left(x_{0}\right)$ and hence we obtain

$$
\begin{aligned}
& \mathfrak{h}_{0}\left(\mathfrak{f}_{1} T_{z}\right)=d\left(z_{0}, \operatorname{aff}\left(\mathfrak{f}_{0,1} T_{x}\right)\right)=d\left(\pi_{\operatorname{aff}\left(\mathfrak{f}_{1} T_{y}\right)}\left(x_{0}\right), \pi_{\text {aff( }\left(\mathfrak{f c}_{0,1} T_{x}\right)}\left(z_{0}\right)\right) \\
& =d\left(\pi_{\mathrm{aff}\left(\mathfrak{f c}_{1} T_{y}\right)}\left(x_{0}\right), \pi_{\mathrm{aff}\left(\mathfrak{f c}_{1} T_{y}\right)}\left(\pi_{\mathrm{aff}\left(\mathfrak{f}_{0,1} T_{x}\right)}\left(z_{0}\right)\right)\right) \\
& \leq d(x_{0}, \underbrace{\pi_{\mathrm{aff}\left(\mathfrak{f}_{0,1} T_{x}\right)}\left(z_{0}\right)}_{=\pi_{\mathrm{aff}\left(f \mathfrak{c}_{0,1} T_{x}\right)}\left(x_{0}\right)}) \\
& =d\left(x_{0}, \operatorname{aff}\left(\mathfrak{f c}_{0,1} T_{x}\right)\right) \\
& =\mathfrak{h}_{0}\left(\mathfrak{f c}_{1} T_{x}\right) \text {. }
\end{aligned}
$$

Now, we deduce with Lemma 2.13

$$
\begin{aligned}
\mathfrak{h}_{1} T_{y} & =\frac{\mathfrak{h}_{0} T_{y}}{\mathfrak{h}_{0}\left(\mathfrak{c}_{1} T_{y}\right)} \mathfrak{h}_{1}\left(\mathfrak{f}_{0} T_{y}\right) \\
& \stackrel{\mathrm{IV}}{=} \frac{\mathfrak{h}_{0} T_{z}}{\mathfrak{h}_{0}\left(\mathfrak{f c}_{1} T_{z}\right)} \mathfrak{h}_{1}\left(\mathfrak{f}_{0} T_{x}\right) \\
& \geq \mathfrak{h}_{0} T_{z} \frac{\mathfrak{h}_{1}\left(\mathfrak{f}_{0} T_{x}\right)}{\mathfrak{h}_{0}\left(\mathfrak{f}_{1} T_{x}\right)} \\
& \geq\left(\mathfrak{h}_{0} T_{x}-d\left(x_{0}, z_{0}\right)\right) \frac{\mathfrak{h}_{1}\left(\mathfrak{f}_{0} T_{x}\right)}{\mathfrak{h}_{0}\left(\mathfrak{f}_{1} T_{x}\right)} .
\end{aligned}
$$

1. Case: $\frac{\mathfrak{h}_{1}\left(\mathfrak{f}_{0} T_{x}\right)}{\mathfrak{h}_{0}\left(\mathfrak{c}_{1} T_{x}\right)} \geq 1$.

We obtain with II.

$$
\mathfrak{h}_{1} T_{y} \geq H-h .
$$

2. Case: $\frac{\mathfrak{h}_{1}\left(\mathfrak{f c}_{0} T_{x}\right)}{\mathfrak{h}_{0}\left(\mathfrak{f c}_{1} T_{x}\right)}<1$.

With Lemma 2.13 and II. we get

$$
\begin{aligned}
\mathfrak{h}_{1} T_{y} & \geq \mathfrak{h}_{0} T_{x} \frac{\mathfrak{h}_{1}\left(\mathfrak{f}_{0} T_{x}\right)}{\mathfrak{h}_{0}\left(\mathfrak{f c}_{1} T_{x}\right)}-d\left(x_{0}, z_{0}\right) \frac{\mathfrak{h}_{1}\left(\mathfrak{f c}_{0} T_{x}\right)}{\mathfrak{h}_{0}\left(\mathfrak{f c}_{1} T_{x}\right)} \\
& >\mathfrak{h}_{1} T_{x}-d\left(x_{0}, z_{0}\right) \\
& \geq H-h .
\end{aligned}
$$

Since, for $i=2, \ldots, m$, the points $x_{i}$ fulfil the same requirements as $x_{1}$, we are able to prove $\mathfrak{h}_{i} T_{y} \geq H-h$ for all $i=1, \ldots, m$ in the same way. So, $T_{y}$ is an $(m, H-h)$-simplex.

Lemma 2.15. Let $C>0,1 \leq m \leq N$ and let $G \subset \mathbb{R}^{N}$ be a finite set so that for all $(m+1)$ simplices $S=\Delta\left(x_{0}, \ldots, x_{m+1}\right) \in G$, there exists some $i \in\{0, \ldots, m+1\}$ so that $\mathfrak{f c}_{i}(S)$ is no $(m, C)$-simplex.

Then there exists some $m$-simplex $T_{z}=\Delta\left(z_{0}, \ldots, z_{m}\right) \in G$ so that for all $a \in G$, there exists some $i \in\{0, \ldots, m\}$ with $d\left(a, \operatorname{aff}\left(\mathfrak{f}_{i}\left(T_{z}\right)\right)<2 C\right.$.

Proof. Since $G$ is finite, we are able to choose $T_{z}=\Delta\left(z_{0}, \ldots, z_{m}\right) \in G$ so that

$$
\mathfrak{v}\left(T_{z}\right)=\max _{w_{0}, \ldots, w_{m} \in G} \mathfrak{v}\left(\Delta\left(w_{0}, \ldots, w_{m}\right)\right) .
$$


We can assume that $T_{z}$ is an $(m, 2 C)$-simplex, otherwise there would exist some $i \in\{0, \ldots, m\}$ with $\mathfrak{h}_{i}\left(T_{z}\right)<2 C$ and so for all $a \in G$ we would obtain

$$
d\left(a, \operatorname{aff}\left(\mathfrak{f}_{i}\left(T_{z}\right)\right)\right) \stackrel{(2.1)}{\leq} d\left(z_{i}, \operatorname{aff}\left(\mathfrak{f}_{i}\left(T_{z}\right)\right)\right)=\mathfrak{h}_{i}\left(T_{z}\right)<2 C .
$$

Now, choose an arbitrary $y_{0} \in G$. Set $S:=\Delta\left(y_{0}, z_{0}, \ldots, z_{m}\right)$. From our assumption we know that one face of $S$ is no $(m, C)$-simplex. Without loss of generality we assume that

$$
T_{y}:=\Delta\left(y_{0}, z_{1}, \ldots, z_{m}\right)
$$

is not an $(m, C)$-simplex (but an $m$-simplex). So there exists some $i \in\{0, \ldots, m\}$ with

$$
\mathfrak{h}_{i}\left(T_{y}\right)<C \text {. }
$$

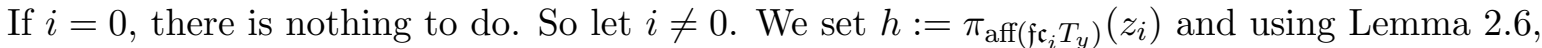
we get

$$
\begin{aligned}
& d\left(h, \operatorname{aff}\left(\mathfrak{f}_{0, i} T_{y}\right)\right)=d\left(h, \pi_{\mathrm{aff}\left(\mathfrak{f}_{0, i} T_{y}\right)}(h)\right) \\
& =d\left(\pi_{\operatorname{aff}\left(\mathfrak{f}_{i} T_{y}\right)}\left(z_{i}\right), \pi_{\operatorname{aff}_{\left(\mathfrak{c}_{0, i} T_{y}\right)}}\left[\pi_{\left.\mathrm{aff}_{(\mathfrak{f}} \mathfrak{c}_{i} T_{y}\right)}\left(z_{i}\right)\right]\right. \\
& =d\left(\pi_{\mathrm{aff}\left(\mathfrak{f}_{i} T_{y}\right)}\left(z_{i}\right), \pi_{\mathrm{aff}\left(\mathfrak{f c}_{i} T_{y}\right)}\left[\pi_{\mathrm{aff}\left(\mathfrak{f}_{0, i} T_{y}\right)}\left(z_{i}\right)\right]\right) \\
& \leq d\left(z_{i}, \operatorname{aff}\left(\mathfrak{f c}_{0, i} T_{y}\right)\right) \text {. }
\end{aligned}
$$

Now, we use Corollary 2.5, with $a_{1}=y_{0}, a_{2}=h \in P_{1}:=\operatorname{aff}\left(\mathfrak{f c}_{i}\left(T_{y}\right)\right), P_{2}:=\operatorname{aff}\left(\mathfrak{f}_{i}\left(T_{z}\right)\right)$, $P_{1} \cap P_{2}=\operatorname{aff}\left(\mathfrak{f}_{0, i}\left(T_{y}\right)\right)$ and obtain

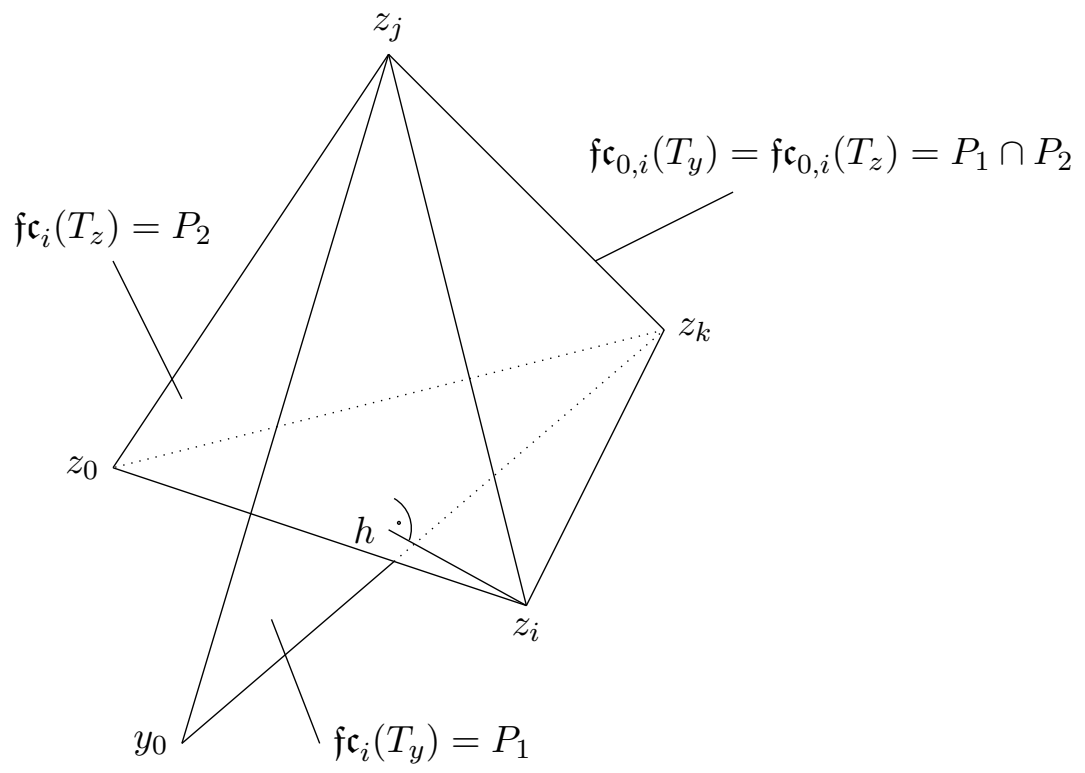

Figure 2.2: The setting for applying Corollary 2.5. 


$$
\begin{aligned}
& \mathfrak{h}_{0}\left(\mathfrak{f}_{i} T_{y}\right)=d\left(y_{0}, \operatorname{aff}\left(\mathfrak{f c}_{0, i}\left(T_{y}\right)\right)\right) \\
& \stackrel{\text { Cor. }}{=}{ }^{2.5} d\left(h, \operatorname{aff}\left(\mathfrak{f}_{0, i}\left(T_{y}\right)\right)\right) \frac{d\left(y_{0}, \operatorname{aff}\left(\mathfrak{f c}_{i}\left(T_{z}\right)\right)\right)}{d\left(h, \operatorname{aff}\left(\mathfrak{f c}_{i}\left(T_{z}\right)\right)\right)} \\
& \stackrel{(2.3)}{\leq} d\left(z_{i}, \operatorname{aff}\left(\mathfrak{f c}_{0, i} T_{y}\right)\right) \frac{\overbrace{d\left(y_{0}, \operatorname{aff}\left(\mathfrak{f c}_{i}\left(T_{z}\right)\right)\right)}^{\frac{(2.1)}{\leq} d\left(z_{i}, \operatorname{aff}\left(\mathfrak{f c}_{i}\left(T_{z}\right)\right)\right)}}{d\left(h, \operatorname{aff}\left(\mathfrak{f c}_{i}\left(T_{z}\right)\right)\right)} \\
& \leq \mathfrak{h}_{i}\left(\mathfrak{f}_{0} T_{y}\right) \frac{d\left(z_{i}, \operatorname{aff}\left(\mathfrak{f}_{i}\left(T_{z}\right)\right)\right)}{d\left(z_{i}, \operatorname{aff}\left(\mathfrak{f c}_{i}\left(T_{z}\right)\right)\right)-d\left(z_{i}, h\right)} \\
& =\mathfrak{h}_{i}\left(\mathfrak{f c}_{0} T_{y}\right)(1+\underbrace{\frac{d\left(z_{i}, h\right)}{d\left(z_{i}, \operatorname{aff}\left(\mathfrak{f c}_{i}\left(T_{z}\right)\right)\right)}-\underbrace{d\left(z_{i}, h\right)}_{\stackrel{(2.2)}{=} \mathfrak{h}_{i}\left(T_{y}\right)<C}}_{=\mathfrak{h}_{i} T_{z} \geq 2 C}) \\
& <2 \mathfrak{h}_{i}\left(\mathfrak{f}_{0} T_{y}\right) .
\end{aligned}
$$

Now, with Lemma 2.13, we have

$$
d\left(y_{0}, \operatorname{aff}\left(\mathfrak{f}_{0}\left(T_{z}\right)\right)\right)=\mathfrak{h}_{0}\left(T_{y}\right)=\mathfrak{h}_{i}\left(T_{y}\right) \frac{\mathfrak{h}_{0}\left(\mathfrak{f}_{i} T_{y}\right)}{\mathfrak{h}_{i}\left(\mathfrak{f c}_{0} T_{y}\right)} \stackrel{(2.2)}{<} 2 C
$$

Lemma 2.16. Let $H>0,1 \leq m \leq N$ and $D \subset \mathbb{R}^{N}$ be a bounded set. Assume that every simplex $S=\Delta\left(y_{0}, \ldots, y_{m}\right) \in D$ is not an $(m, H)$-simplex. Then there exists some $l \in \mathbb{N} \cup\{0\}$, $l \leq m-1$ and $x_{0}, \ldots, x_{l} \in \bar{D}$ so that $\bar{D} \subset U_{H}\left(\operatorname{aff}\left(x_{0}, \ldots, x_{l}\right)\right)=\left\{x \in \mathbb{R}^{N} \mid d\left(x, \operatorname{aff}\left(x_{0}, \ldots, x_{l}\right) \leq\right.\right.$ $H\}$.

Proof. We assume $\# D \geq 2$, otherwise the statement is trivial. We set

$$
0 \leq l:=\max (\{\tilde{l} \in \mathbb{N} \mid \exists(\tilde{l}, H) \text {-simplex in } D\} \cup\{0\}) \leq m-1 .
$$

If $l=0$, we have $\bar{D} \subset U_{H}\left(\operatorname{aff}\left(x_{0}\right)\right)=B\left(x_{0}, H\right)$ for an arbitrary $x_{0} \in D$.

Now suppose $l \geq 1$. Since $D$ is bounded, we get

$$
0<K:=\sup _{\tilde{x}_{0}, \ldots, \tilde{x}_{l} \in D} \mathfrak{v}\left(\triangle\left(\tilde{x}_{0}, \ldots, \tilde{x}_{l}\right)\right)<\infty
$$

Let $x_{0}^{i}, \ldots, x_{l}^{i} \in D$ with $\lim _{i \rightarrow \infty} \mathfrak{v}\left(\triangle\left(x_{0}^{i}, \ldots, x_{l}^{i}\right)\right)=K$. Since $D$ is bounded, we can choose some subsequence of $x_{j}^{i}$ so that $\lim _{i \rightarrow \infty} x_{j}^{i}=x_{j} \in \bar{D}$ for all $j=0, \ldots, l$ and the limit fulfils $\mathfrak{v}\left(\triangle\left(x_{0}, \ldots, x_{l}\right)\right)=K$, since the Gram determinant is a continuous function on $\left(\mathbb{R}^{N}\right)^{l}$ and $\sqrt{\operatorname{Gram}\left(x_{1}-x_{0}, \ldots, x_{l}-x_{0}\right)}=\mathfrak{v}\left(\triangle\left(x_{0}, \ldots, x_{l}\right)\right)$ (cf. Definition 2.11).

Now we choose some arbitrary $x_{l+1} \in \bar{D}$ and some sequence $x_{l+1}^{i} \in D$ with $\lim _{i \rightarrow \infty} x_{l+1}^{i}=$ $x_{l+1}$. From (2.4) we know that $\triangle\left(x_{0}^{i}, \ldots, x_{l+1}^{i}\right)$ is not an $(l+1, H)$-simplex for all $i \in \mathbb{N}$.

Let $\varepsilon>0$. There exists some $i \in \mathbb{N}$ so that $\left|x_{j}^{i}-x_{j}\right|<\frac{\varepsilon}{l+2}$ for all $j \in\{0, \ldots, l\}$. Due to Lemma 2.14, the simplex $\triangle\left(x_{0}, x_{1}^{i}, \ldots, x_{l+1}^{i}\right)$ is not an $\left(l+1, H+\frac{\varepsilon}{l+2}\right)$-simplex. Repeating 
this argument, implies that $T:=\triangle\left(x_{0}, \ldots, x_{l+1}\right)$ is not an $(l+1, H+\varepsilon)$-simplex. Hence there exists some $\tilde{l} \in\{0, \ldots, l+1\}$ so that $\mathfrak{h}_{\tilde{l}}(T)<H+\varepsilon$. We obtain with Remark 2.12

$$
d\left(x_{l+1}, \operatorname{aff}\left(x_{0}, \ldots, x_{l}\right)\right)=\mathfrak{h}_{l+1}(T)=\mathfrak{h}_{\tilde{l}}(T) \frac{\mathfrak{v}\left(\mathfrak{f}_{\tilde{l}}(T)\right)}{\mathfrak{v}\left(\mathfrak{f}_{l+1}(T)\right)} \stackrel{(2.5)}{\leq} \mathfrak{h}_{\tilde{l}}(T) \frac{K}{K}<H+\varepsilon
$$

It follows that $\bar{D} \subset U_{H}\left(\operatorname{aff}\left(x_{0}, \ldots, x_{l}\right)\right)$ because $x_{l+1} \in \bar{D}$ and $\varepsilon>0$ were independently and arbitrarily chosen.

Lemma 2.17. Let $1 \leq m \leq N-1, B$ be a closed ball in $\mathbb{R}^{N}$ and $F \subset B$ be a $\mathcal{H}^{m}$-measurable set with $\mathcal{H}^{m}(F)=\infty$. There exists a small constant $0<\sigma=\sigma(F, B) \leq \frac{\operatorname{diam} B}{2}$ and some $(m+1,(m+3) \sigma)$-simplex $T=\Delta\left(x_{0}, \ldots, x_{m+1}\right) \in B$ with $\mathcal{H}^{m}\left(B\left(x_{0}, \sigma\right) \cap F\right)=\infty$ and $\mathcal{H}^{m}\left(B\left(x_{i}, \sigma\right) \cap F\right)>0$ for all $i \in\{1, \ldots, m+1\}$.

Proof. We set $\mu:=\mathcal{H}^{m} \mathrm{~L} F$.

1. Claim: There exists some $x_{0} \in B$ with $\mu\left(B\left(x_{0}, h\right)\right)=\infty$ for all $h>0$.

Assume that there exists some $r>0$ so that, for all $x \in B$, we have $\mu(B(x, r))<\infty$. With a simple covering argument, we obtain $\mu(B)<\infty$ in contradiction to our requirements. So, for every $i \in \mathbb{N}$ and $r_{i}:=\frac{1}{i}$, we get some $y_{i} \in B$ with $\mu\left(B\left(y_{i}, r_{i}\right)\right)=\infty$. Since $B$ is compact, there exists some convergent subsequence $\left(y_{i}\right)$ and some $x_{0} \in B$ with $\lim _{i \rightarrow \infty} y_{i}=x_{0}$.

Let $h>0$. For some large $i$ we obtain $d\left(x_{0}, y_{i}\right) \leq \frac{h}{2}$ and $r_{i} \leq \frac{h}{2}$ so that $\mu\left(B\left(x_{0}, h\right)\right) \geq$ $\mu\left(B\left(y_{i}, r_{i}\right)\right)=\infty$.

2. Claim: There exists some $c_{1}>0$ and some $x_{1} \in B$ so that the simplex $T_{1}:=\Delta\left(x_{0}, x_{1}\right)$ fulfils $\mathfrak{h}_{1}\left(T_{1}\right) \geq c_{1}$ and $\mu\left(B\left(x_{1}, h\right)\right)>0$ for all $h>0$.

Assume that we have $\mu\left(B \backslash B\left(x_{0}, r\right)\right)=0$ for all $r>0$. We get $\mu(B)=0$ because we are able to cover $B$ with null sets. This is a contradiction, so there exists some $c_{1}>0$ with $\mu\left(B \backslash \stackrel{\circ}{B}\left(x_{0}, c_{1}\right)\right)>0$. With Lemma A.6, there exists some $x_{1} \in B \backslash \stackrel{\circ}{B}\left(x_{0}, c_{1}\right)$ with $\mu\left(B\left(x_{1}, h\right)\right)>0$ for all $h>0$. So the simplex $T_{1}$ fulfils $\mathfrak{h}_{1}\left(T_{1}\right)=d\left(x_{0}, x_{1}\right) \geq c_{1}$.

3. Claim: We assume that we already have $c_{l}>0$ and a simplex $T_{l}=\Delta\left(x_{0}, \ldots, x_{l}\right) \in \mathbb{R}^{N}$ with $\mathfrak{h}_{l}\left(T_{l}\right) \geq c_{l}$ and $\mu\left(B\left(x_{i}, h\right)\right)>0$ for all $i \in\{0, \ldots, l\}$ and $h>0$ where $l \leq m$. Then there exists some $0<c_{l+1}<\frac{c_{l}}{2}$ and some $x_{l+1} \in B$ so that $T_{l+1}:=\Delta\left(x_{0}, \ldots, x_{l+1}\right)$ fulfils $\mathfrak{h}_{l+1}\left(T_{l+1}\right) \geq c_{l+1}$ and $\mu\left(B\left(x_{l+1}, h\right)\right)>0$ for all $h>0$.

Assume that $\mu\left(B\left(x_{0}, \frac{c_{l}}{2}\right) \backslash \stackrel{\circ}{U}_{c}\left(\operatorname{aff}\left(x_{0}, \ldots, x_{l}\right)\right)\right)=0$ for all $c>0$. We set $D_{i}:=B\left(x_{0}, \frac{c_{l}}{2}\right) \backslash$ $\stackrel{\circ}{U}_{\frac{1}{i}}\left(\operatorname{aff}\left(x_{0}, \ldots, x_{l}\right)\right)$ and obtain a contradiction

$$
\infty=\mu\left(B\left(x_{0}, \frac{c_{l}}{2}\right)\right) \leq \underbrace{\mu\left(\operatorname{aff}\left(x_{0}, \ldots, x_{l}\right) \cap B\left(x_{0}, \frac{c_{l}}{2}\right)\right)}_{\left\{\begin{array}{l}
=0, \\
\leq \omega_{m}\left(\frac{c_{l}}{2}\right)^{m}<\infty,
\end{array} \quad \text { if } l=m\right.}+\underbrace{\sum_{i \in \mathbb{N}} \mu\left(D_{i}\right)}_{=0}<\infty .
$$

So there exists some $0<c_{l+1}<\frac{c_{l}}{2}$ with $\mu\left(\left(F \cap B\left(x_{0}, \frac{c_{l}}{2}\right)\right) \backslash \stackrel{\circ}{U}_{c_{l+1}}\left(\operatorname{aff}\left(x_{0}, \ldots, x_{l}\right)\right)\right)>0$ and, with Lemma A.6, there exists some $x_{l+1} \in F \subset B$ so that $T_{l+1}:=\Delta\left(x_{0}, \ldots, x_{l+1}\right)$ fulfils $\mathfrak{h}_{l+1}\left(T_{l+1}\right) \geq c_{l+1}$ and $\mu\left(B\left(x_{l+1}, h\right)\right)>0$ for all $h>0$. 
4. Claim: There exists some constant $c>0$ so that $T:=T_{m+1}$ is an $(m+1, c)$-simplex. Assume that there exists some $i \in\{0, \ldots, m+1\}$ so that $\mathfrak{h}_{i}(T)=0$. This implies

$$
0=\mathfrak{h}_{i}(T) \mathfrak{v}\left(\mathfrak{f}_{i}(T)\right)=\mathfrak{v}(T)=\mathfrak{h}_{m+1}\left(T_{m+1}\right) \mathfrak{v}\left(T_{m}\right)=\prod_{i=1}^{m+1} \underbrace{\mathfrak{h}_{i}\left(T_{i}\right)}_{\geq c_{i}>0}>0 .
$$

This is a contradiction.

To conclude the proof set $\sigma:=\frac{c}{m+3}$.

\subsection{Angles between affine subspaces}

Definition 2.18. 1. For $G_{1}, G_{2} \in G(N, m)$, we define

$$
\Varangle\left(G_{1}, G_{2}\right):=\left\|\pi_{G_{1}}-\pi_{G_{2}}\right\|,
$$

where the right hand side is the usual norm of the linear map $\pi_{G_{1}}-\pi_{G_{2}}$.

2. For $P_{1}, P_{2} \in \mathcal{P}(N, m)$, we define

$$
\Varangle\left(P_{1}, P_{2}\right):=\Varangle\left(P_{1}-\pi_{P_{1}}(0), P_{2}-\pi_{P_{2}}(0)\right) .
$$

Lemma 2.19. For $P_{1}, P_{2} \in \mathcal{P}(N, m)$ and $w \in \mathbb{R}^{N}$, we have

$$
\Varangle\left(P_{1}, P_{2}\right)=\Varangle\left(P_{1}, P_{2}+w\right) .
$$

Proof. Using Lemma 2.1, we get

$$
P_{2}+w-\pi_{P_{2}+w}(0)=P_{2}-\pi_{P_{2}}(-w)=P_{2}-\pi_{P_{2}}(0)
$$

and obtain

$$
\Varangle\left(P_{1}, P_{2}+w\right)=\left\|\pi_{P_{1}-\pi_{P_{1}}(0)}-\pi_{P_{2}+w-\pi_{P_{2}+w}(0)}\right\|=\Varangle\left(P_{1}, P_{2}\right) .
$$

Lemma 2.20 (Triangle inequality). We have for $P_{1}, P_{2}, P_{3} \in \mathcal{P}(N, m)$

$$
\Varangle\left(P_{1}, P_{3}\right) \leq \Varangle\left(P_{1}, P_{2}\right)+\Varangle\left(P_{2}, P_{3}\right) .
$$

Proof. This statement follows directly from the triangle inequality for the norm of linear maps

$$
\begin{aligned}
\Varangle\left(P_{1}, P_{3}\right) & =\left\|\pi_{P_{1}-\pi_{P_{1}}(0)}-\pi_{P_{3}-\pi_{P_{3}}(0)}\right\| \\
& \leq\left\|\pi_{P_{1}-\pi_{P_{1}}(0)}-\pi_{P_{2}-\pi_{P_{2}}(0)}\right\|+\left\|\pi_{P_{2}-\pi_{P_{2}}(0)}-\pi_{P_{3}-\pi_{P_{3}}(0)}\right\| \\
& =\Varangle\left(P_{1}, P_{2}\right)+\Varangle\left(P_{2}, P_{3}\right) .
\end{aligned}
$$

Remark. The angle $\Varangle$ is a metric on the Grassmannian $G(N, m)$ but not on $\mathcal{P}(N, m)$ because for $P \in \mathcal{P}(N, m)$, there exists some $w \in \mathbb{R}^{N}$ so that $\Varangle(P, P-w)=0$, but $P \neq P-w$. 
Lemma 2.21. Let $U \in G(N, m)$ and $v \in \mathbb{R}^{N}$ with $|v|=\left|\pi_{U}(v)\right|$. Then we have $v=\pi_{U}(v)$. Proof. We have

$$
\left|\pi_{U}(v)\right|^{2}=|v|^{2}=\left|\pi_{U}(v)+\pi_{U}^{\perp}(v)\right|^{2}=\left|\pi_{U}(v)\right|^{2}+\left|\pi_{U}^{\perp}(v)\right|^{2}
$$

and so $\pi_{U}^{\perp}(v)=0$ which implies $v=\pi_{U}(v)+\pi_{U}^{\perp}(v)=\pi_{U}(v)$.

Lemma 2.22. Let $P_{1}, P_{2} \in G(N, m)$ and $v \in \mathbb{S}^{N-1}$ with $0<\Varangle\left(P_{1}, P_{2}\right)=\left|\pi_{P_{1}}(v)-\pi_{P_{2}}(v)\right|$. Then $v \in\left(P_{1} \cap P_{2}\right)^{\perp} \cap \operatorname{span}\left(P_{1}, P_{2}\right)$.

Proof. If $v \in P_{1} \cap P_{2}$, we get $\Varangle\left(P_{1}, P_{2}\right)=0$, so $v \notin P_{1} \cap P_{2}$. We have with Lemma 2.6

$$
\begin{aligned}
\Varangle\left(P_{1}, P_{2}\right) & =\left|\pi_{P_{1}}(v)-\pi_{P_{2}}(v)\right| \\
& =\mid \pi_{P_{1}}\left(\pi_{\mathrm{span}\left(P_{1}, P_{2}\right)}(v)\right)-\pi_{P_{2}\left(\pi_{\mathrm{span}\left(P_{1}, P_{2}\right)}(v)\right) \mid} \\
& =\underbrace{\left|\pi_{\mathrm{span}\left(P_{1}, P_{2}\right)}(v)\right|}_{\leq 1}\left|\pi_{P_{1}}(\underbrace{\pi_{\mathrm{span}\left(P_{1}, P_{2}\right)}(v)}_{=: w \in \operatorname{span}\left(P_{1}, P_{2}\right)})-\pi_{P_{2}}\left(\frac{\pi_{\mathrm{span}\left(P_{1}, P_{2}\right)}(v)}{\left|\pi_{\mathrm{span}\left(P_{1}, P_{2}\right)}(v)\right|}\right)\right| \\
& \leq\left|\pi_{P_{1}}(w)-\pi_{P_{2}}(v)\right| \\
& \leq \Varangle\left(P_{1}, P_{2}\right) .
\end{aligned}
$$

Hence $\left|\pi_{\operatorname{span}\left(P_{1}, P_{2}\right)}(v)\right|=1=|v|$ and so, with Lemma 2.21, we conclude $v=\pi_{\operatorname{span}\left(P_{1}, P_{2}\right)}(v) \in$ $\operatorname{span}\left(P_{1}, P_{2}\right)$.

We choose an orthonormal basis of $P_{1} \cap P_{2}=\operatorname{span}\left(o_{1}, \ldots, o_{l}\right)$ where $l=\operatorname{dim}\left(P_{1} \cap P_{2}\right)$ and extend this to orthonormal bases of $P_{1}$ and of $P_{2}$

$$
\begin{aligned}
& P_{1}=\operatorname{span}\left(o_{1}, \ldots, o_{l}, o_{l+1}^{1}, \ldots, o_{m}^{1}\right) \\
& P_{2}=\operatorname{span}\left(o_{1}, \ldots, o_{l}, o_{l+1}^{2}, \ldots, o_{m}^{2}\right) .
\end{aligned}
$$

We have $v \in \operatorname{span}\left(P_{1}, P_{2}\right)=\operatorname{span}\left(o_{1}, \ldots, o_{l}, o_{l+1}^{1}, \ldots, o_{m}^{1}, o_{l+1}^{2}, \ldots, o_{m}^{2}\right)$, so there exists some

$$
\alpha_{1}, \ldots, \alpha_{l}, \alpha_{l+1}^{1}, \ldots, \alpha_{m}^{1}, \alpha_{l+1}^{2}, \ldots, \alpha_{m}^{2} \in \mathbb{R}
$$

with

$$
v=\underbrace{\sum_{i=1}^{l} \alpha_{i} o_{i}}_{\in P_{1} \cap P_{2}}+\underbrace{\sum_{i=l+1}^{m}\left(\alpha_{i}^{1} o_{i}^{1}+\alpha_{i}^{2} o_{i}^{2}\right)}_{=: \bar{v} \in Q}
$$

where $Q:=\left(P_{1} \cap P_{2}\right)^{\perp} \cap \operatorname{span}\left(P_{1}, P_{2}\right)$. We obtain

$$
\begin{aligned}
\Varangle\left(P_{1}, P_{2}\right) & =\left|\pi_{P_{1}}(v)-\pi_{P_{2}}(v)\right| \\
& =\left|\pi_{P_{1}}(\bar{v})-\pi_{P_{2}}(\bar{v})\right| \\
& =\left|\pi_{P_{1}}\left(\pi_{Q}(v)\right)-\pi_{P_{2}}\left(\pi_{Q}(v)\right)\right| \\
& =\underbrace{\left|\pi_{Q}(v)\right|}_{\leq 1}\left|\pi_{P_{1}}(\underbrace{\frac{\pi_{Q}(v)}{\left|\pi_{Q}(v)\right|}}_{=: w \in Q})-\pi_{P_{2}}\left(\frac{\pi_{Q}(v)}{\left|\pi_{Q}(v)\right|}\right)\right| \\
& \leq\left|\pi_{P_{1}}(w)-\pi_{P_{2}}(w)\right| \\
& \leq \Varangle\left(P_{1}, P_{2}\right)
\end{aligned}
$$


and so $\left|\pi_{Q}(v)\right|=1=|v|$. We conclude with Lemma $2.21 v=\pi_{Q}(v) \in Q$.

Lemma 2.23. Let $P_{1}, P_{2} \in G(N, m)$ with $\Varangle\left(P_{1}, P_{2}\right)<1$ and $x, y \in P_{1}$. We have

$$
d(x, y) \leq \frac{1}{1-\Varangle\left(P_{1}, P_{2}\right)} d\left(\pi_{P_{2}}(x), \pi_{P_{2}}(y)\right)
$$

and

$$
d\left(\pi_{P_{2}}^{\perp}(x), \pi_{P_{2}}^{\perp}(y)\right) \leq \frac{\Varangle\left(P_{1}, P_{2}\right)}{1-\Varangle\left(P_{1}, P_{2}\right)} d\left(\pi_{P_{2}}(x), \pi_{P_{2}}(y)\right) .
$$

Proof. With $z:=\frac{x-y}{|x-y|} \in P_{1}$ we get

$$
\begin{aligned}
\left|\pi_{P_{2}}^{\perp}(x)-\pi_{P_{2}}^{\perp}(y)\right| & =|x-y|\left|\pi_{P_{2}}^{\perp}(z)+\pi_{P_{2}}(z)-\pi_{P_{2}}(z)\right| \\
& =|x-y||\underbrace{z}_{=\pi_{P_{1}}(z)}-\pi_{P_{2}}(z)| \\
& \leq|x-y| \Varangle\left(P_{1}, P_{2}\right),
\end{aligned}
$$

so that

$$
\begin{aligned}
d(x, y) & \leq d\left(\pi_{P_{2}}(x), \pi_{P_{2}}(y)\right)+d\left(\pi_{P_{2}}^{\perp}(x), \pi_{P_{2}}^{\perp}(y)\right) \\
& \leq d\left(\pi_{P_{2}}(x), \pi_{P_{2}}(y)\right)+d(x, y) \Varangle\left(P_{1}, P_{2}\right)
\end{aligned}
$$

and finally

$$
d(x, y) \leq \frac{1}{1-\Varangle\left(P_{1}, P_{2}\right)} d\left(\pi_{P_{2}}(x), \pi_{P_{2}}(y)\right) .
$$

The first and the last estimate together give

$$
d\left(\pi_{P_{2}}^{\perp}(x), \pi_{P_{2}}^{\perp}(y)\right) \leq \frac{\Varangle\left(P_{1}, P_{2}\right)}{1-\Varangle\left(P_{1}, P_{2}\right)} d\left(\pi_{P_{2}}(x), \pi_{P_{2}}(y)\right) .
$$

Corollary 2.24. Let $P_{1}, P_{2} \in \mathcal{P}(N, m)$ with $\Varangle\left(P_{1}, P_{2}\right)<1$ and $x, y \in P_{1}$. We have

$$
d(x, y) \leq \frac{1}{1-\Varangle\left(P_{1}, P_{2}\right)} d\left(\pi_{P_{2}}(x), \pi_{P_{2}}(y)\right)
$$

and

$$
d\left(\pi_{P_{2}}^{\perp}(x), \pi_{P_{2}}^{\perp}(y)\right) \leq \frac{\Varangle\left(P_{1}, P_{2}\right)}{1-\Varangle\left(P_{1}, P_{2}\right)} d\left(\pi_{P_{2}}(x), \pi_{P_{2}}(y)\right) .
$$

Proof. Choose $t_{1} \in P_{1}, t_{2} \in P_{2}$. We obtain $P_{1}-t_{1}, P_{2}-t_{2} \in G(N, m)$. Using Lemma 2.23, Lemma 2.1 and Lemma 2.19, we get

$$
\begin{aligned}
d(x, y) & =d\left(x-t_{1}, y-t_{1}\right) \\
& \leq \frac{1}{1-\Varangle\left(P_{1}-t_{1}, P_{2}-t_{2}\right)} d\left(\pi_{P_{2}-t_{2}}\left(x-t_{1}\right), \pi_{P_{2}-t_{2}}\left(y-t_{1}\right)\right) \\
& =\frac{1}{1-\Varangle\left(P_{1}, P_{2}\right)} d\left(\pi_{P_{2}}(x), \pi_{P_{2}}(y)\right)
\end{aligned}
$$

and, analogously, we get the second estimate. 
Corollary 2.25. Let $P_{1} \in \mathcal{P}(N, m), P_{2} \in G(N, m)$ and $\Varangle\left(P_{1}, P_{2}\right)<1$. There exists some affine map $a: P_{2} \rightarrow P_{2}^{\perp}$ with $G(a)=P_{1}$, where $G(a)$ is the graph of the map $a$, and $a$ is Lipschitz continuous with Lipschitz constant $\frac{\Varangle\left(P_{1}, P_{2}\right)}{1-\Varangle\left(P_{1}, P_{2}\right)}$.

Proof. According to Corollary 2.24, the projection $\pi_{P_{2}}: P_{1} \rightarrow P_{2}$ is injective, so obviously the desired affine map exists, i.e., $a(y)=\pi_{P_{2}}^{\perp}\left(\left.\pi_{P_{2}}^{-1}\right|_{P_{1}}(y)\right)$. Now let $u, v \in P_{2}$. Using Corollary 2.24 again, we get $d(a(u), a(v))=d\left(\pi_{P_{2}}^{\perp}(a(u)+u), \pi_{P_{2}}^{\perp}(a(v)+v)\right) \leq \frac{\Varangle\left(P_{1}, P_{2}\right)}{1-\Varangle\left(P_{1}, P_{2}\right)} d(u, v)$.

Corollary 2.26. Let $P_{1}, P_{2} \in G(N, m)$ and $o_{1}, \ldots, o_{m}$ be an orthonormal basis of $P_{1}$. If $d\left(o_{i}, P_{2}\right) \leq \tilde{\sigma} \leq \tilde{\sigma}_{1}:=10^{-1}\left(10^{m}+1\right)^{-1}$, then $\Varangle\left(P_{1}, P_{2}\right) \leq 4 m\left(10^{m}+1\right) \tilde{\sigma}$.

Proof. For $i=1, \ldots, m$, set $h_{i}:=\pi_{P_{2}}\left(o_{i}\right)$ and use Lemma 2.3 from [SvdM13b].

For $x, y \in \mathbb{R}^{N}$, we set $\langle x, y\rangle$ to be the usual scalar product in $\mathbb{R}^{N}$.

Lemma 2.27. Let $C, \hat{C} \geq 1, t>0$ and $S=\Delta\left(y_{0}, \ldots, y_{m}\right)$ an $\left(m, \frac{t}{C}\right)$-simplex with $S \subset$ $B(x, \hat{C} t), x \in \mathbb{R}^{N}$. There exists $\gamma_{l, r} \in \mathbb{R}$ so that

$$
o_{l}:=\sum_{r=1}^{l} \gamma_{l, r}\left(y_{r}-y_{0}\right)
$$

is an orthonormal basis of $\operatorname{span}\left(y_{1}-y_{0}, \ldots, y_{m}-y_{0}\right)$ and

$$
\left|\gamma_{l, r}\right| \leq(2 l C \hat{C})^{l} \frac{C}{t} \leq(2 m C \hat{C})^{m} \frac{C}{t}
$$

for all $1 \leq l \leq m$ and $1 \leq r \leq l$.

Proof. Since $S \subset B(x, \hat{C} t)$, we have for $i, j \in\{0, \ldots, m\}$

$$
d\left(y_{i}, y_{j}\right) \leq 2 \hat{C} t \text {. }
$$

Now we set

$$
z_{i}:=y_{i}-y_{0}
$$

for all $i=0, \ldots, m$, and $R:=\Delta\left(z_{0}, \ldots, z_{m}\right)=S-y_{0}$. We obtain for $i \in\{1, \ldots, m\}$ ( $S$ is an $\left(m, \frac{t}{C}\right)$-simplex $)$

$$
d\left(z_{i}, \operatorname{aff}\left(z_{0}, \ldots, z_{i-1}\right)\right) \geq \mathfrak{h}_{i}(R)=\mathfrak{h}_{i}(S) \geq \frac{t}{C} .
$$

Due to $\mathfrak{h}_{i}(R) \geq \frac{t}{C}>0$, we have that $\left(z_{1}, \ldots, z_{m}\right)$ are linearly independent. So with the Gram-Schmidt process we are able to define some orthonormal basis of the $m$-dimensional linear $\operatorname{subspace} \operatorname{span}\left(z_{1}, \ldots, z_{m}\right)$

$$
\begin{aligned}
o_{1} & :=\frac{z_{1}}{\left|z_{1}\right|}, \\
o_{l+1} & :=\frac{z_{l+1}-\sum_{i=1}^{l}\left\langle z_{l+1}, o_{i}\right\rangle o_{i}}{\left|z_{l+1}-\sum_{i=1}^{l}\left\langle z_{l+1}, o_{i}\right\rangle o_{i}\right|}=\frac{z_{l+1}-\sum_{i=1}^{l}\left\langle z_{l+1}, o_{i}\right\rangle o_{i}}{d\left(z_{l+1}, \operatorname{aff}\left(z_{0}, \ldots, z_{l}\right)\right)} .
\end{aligned}
$$


Now we prove by induction that there exists $\gamma_{l, r} \in \mathbb{R}$ with

$$
o_{l}=\sum_{r=1}^{l} \gamma_{l, r}\left(y_{r}-y_{0}\right)
$$

and

$$
\left|\gamma_{l, r}\right| \leq(2 l C \hat{C})^{l} \frac{C}{t}
$$

for all $1 \leq l \leq m$ and $1 \leq r \leq l$. We have that $o_{1}=\frac{y_{1}-y_{0}}{\left|y_{1}-y_{0}\right|}$, so set

$$
\gamma_{1,1}:=\frac{1}{\left|y_{1}-y_{0}\right|}=\frac{1}{d\left(z_{1}, z_{0}\right)} \stackrel{(2.7)}{\leq} \frac{C}{t}
$$

Now let $1 \leq l \leq m$. We assume that, for all $i \in\{1, \ldots, l\}, j \in\{1, \ldots, i\}$, we have $\gamma_{i, j} \in \mathbb{R}$ with

$$
o_{i}=\sum_{r=1}^{i} \gamma_{i, r}\left(y_{r}-y_{0}\right)
$$

and

$$
\left|\gamma_{i, j}\right| \leq(2 l C \hat{C})^{i} \frac{C}{t}
$$

We obtain

$$
\begin{aligned}
o_{l+1} & =\underbrace{\frac{1}{d\left(z_{l+1}, \operatorname{aff}\left(z_{0}, \ldots, z_{l}\right)\right)}}_{=: \gamma_{l+1, l+1}}\left(\left(y_{l+1}-y_{0}\right)-\sum_{i=1}^{l}\left\langle\left(y_{l+1}-y_{0}\right), o_{i}\right\rangle o_{i}\right) \\
& =\gamma_{l+1, l+1}\left(y_{l+1}-y_{0}\right)+\sum_{i=1}^{l} \frac{\left\langle\left(y_{l+1}-y_{0}\right), o_{i}\right\rangle}{d\left(z_{l+1}, \operatorname{aff}\left(z_{0}, \ldots, z_{l}\right)\right)} \sum_{r=1}^{i} \gamma_{i, r}\left(y_{r}-y_{0}\right) \\
& =\gamma_{l+1, l+1}\left(y_{l+1}-y_{0}\right)+\sum_{i=1}^{l} \sum_{r=1}^{i} \frac{\left\langle\left(y_{l+1}-y_{0}\right), o_{i}\right\rangle}{d\left(z_{l+1}, \operatorname{aff}\left(z_{0}, \ldots, z_{l}\right)\right)} \gamma_{i, r}\left(y_{r}-y_{0}\right) \\
& =\gamma_{l+1, l+1}\left(y_{l+1}-y_{0}\right)+\sum_{r=1}^{l} \underbrace{\sum_{i=r}^{l} \frac{\left\langle\left(y_{l+1}-y_{0}\right), o_{i}\right\rangle}{d\left(z_{l+1}, \operatorname{aff}\left(z_{0}, \ldots, z_{l}\right)\right)} \gamma_{i, r}}_{=: \gamma_{l+1, r}}\left(y_{r}-y_{0}\right) \\
& =\sum_{r=1}^{l+1} \gamma_{l+1, r}\left(y_{r}-y_{0}\right) .
\end{aligned}
$$

If $r=l+1$, we have

$$
\left|\gamma_{l+1, r}\right|=\frac{1}{d\left(z_{l+1}, \operatorname{aff}\left(z_{0}, \ldots, z_{l}\right)\right)} \stackrel{(2.7)}{\leq} \frac{C}{t}
$$


and if $1 \leq r \leq l$, we get

$$
\begin{aligned}
\left|\gamma_{l+1, r}\right| & \leq \sum_{i=r}^{l} \frac{\left|\left\langle\left(y_{l+1}-y_{0}\right), o_{i}\right\rangle\right|}{d\left(z_{l+1}, \operatorname{aff}\left(z_{0}, \ldots, z_{l}\right)\right)}\left|\gamma_{i, r}\right| \\
& \leq \sum_{i=r}^{l} \frac{C\left|y_{l+1}-y_{0}\right|}{t}(2 l C \hat{C})^{r} \frac{C}{t} \\
& \stackrel{(2.6)}{\leq}(2 l C \hat{C})^{l+1} \frac{C}{t} \\
& <(2(l+1) C \hat{C})^{l+1} \frac{C}{t} .
\end{aligned}
$$

Lemma 2.28. Let $C, \hat{C} \geq 1, t>0,0<\sigma \leq\left(10\left(10^{m}+1\right) m C(2 m C \hat{C})^{m}\right)^{-1}, P_{1}, P_{2} \in$ $\mathcal{P}(N, m)$ and $S=\Delta\left(y_{0}, \ldots, y_{m}\right) \subset P_{1}$ an $\left(m, \frac{t}{C}\right)$-simplex with $S \subset B(x, \hat{C} t), x \in \mathbb{R}^{N}$ and $d\left(y_{i}, P_{2}\right) \leq t \sigma$ for all $i \in\{0, \ldots, m\}$. It follows that

$$
\Varangle\left(P_{1}, P_{2}\right) \leq 4 m\left(10^{m}+1\right)\left(m C(2 m C \hat{C})^{m}\right) \sigma .
$$

Proof. With Lemma 2.27, there exists some orthonormal basis $\left(o_{1}, \ldots, o_{m}\right)$ of $\operatorname{span}\left(y_{1}-y_{0}, \ldots, y_{m}-y_{0}\right)$ and there exists $\gamma_{l, r} \in \mathbb{R}$ with

$$
o_{l}=\sum_{r=1}^{l} \gamma_{l, r}\left(y_{r}-y_{0}\right)
$$

and

$$
\left|\gamma_{l, r}\right| \leq(2 m C \hat{C})^{m} \frac{C}{t}
$$

for all $1 \leq l \leq m$ and $1 \leq r \leq l$. We obtain for $1 \leq l \leq m$

$$
\begin{aligned}
d\left(o_{l}, P_{2}-y_{0}\right) & =\left|\sum_{r=1}^{l} \gamma_{l, r}\left(y_{r}-y_{0}\right)-\pi_{P_{2}-y_{0}}\left(\sum_{r=1}^{l} \gamma_{l, r}\left(y_{r}-y_{0}\right)\right)\right| \\
& \leq \sum_{r=1}^{l}\left|\gamma_{l, r}\right| d\left(y_{r}-y_{0}, P_{2}-y_{0}\right) \\
& \leq m C(2 m C \hat{C})^{m} \sigma .
\end{aligned}
$$

Setting $\tilde{\sigma}=m C(2 m C \hat{C})^{m} \sigma \leq \frac{1}{10\left(10^{m}+1\right)}$ we get with Corollary 2.26

$$
\Varangle\left(P_{1}, P_{2}\right)=\Varangle\left(P_{1}-y_{0}, P_{2}-y_{0}\right) \leq 4 m\left(10^{m}+1\right)\left(m C(2 m C \hat{C})^{m}\right) \sigma .
$$


Lemma 2.29. Let $\sigma>0, t \geq 0, P_{1}, P_{2} \in \mathcal{P}(N, m)$ with $\Varangle\left(P_{1}, P_{2}\right) \leq \sigma$ and assume that there exists $p_{1} \in P_{1}, p_{2} \in P_{2}$ with $d\left(p_{1}, p_{2}\right) \leq t \sigma$. Then we have for every $w \in P_{1}$

$$
d\left(w, P_{2}\right) \leq \sigma\left(d\left(w, p_{1}\right)+t\right) .
$$

Proof. For $w \in P_{1}$, set $\tilde{w}:=w-p_{1} \in P_{1}-p_{1}$. We obtain

$$
\begin{aligned}
d\left(w, P_{2}\right) & =d\left(\tilde{w}, P_{2}-p_{1}\right) \\
& \leq d\left(\tilde{w}, P_{2}-p_{2}\right)+d\left(P_{2}-p_{2}, P_{2}-p_{1}\right) \\
& \leq|\tilde{w}||\underbrace{\frac{\tilde{w}}{|\tilde{w}|}}_{=\pi_{P_{1}-p_{1}\left(\frac{\tilde{w}}{|\tilde{w}|}\right)}}-\pi_{P_{2}-p_{2}}\left(\frac{\tilde{w}}{|\tilde{w}|}\right)|+d\left(p_{1}, p_{2}\right) \\
& \leq\left|w-p_{1}\right| \underbrace{\Varangle\left(P_{1}-p_{1}, P_{2}-p_{2}\right)}_{=\Varangle\left(P_{1}, P_{2}\right) \leq \sigma}+t \sigma \\
& \leq \sigma\left(d\left(w, p_{1}\right)+t\right) .
\end{aligned}
$$

Lemma 2.30. For $P_{1}, P_{2} \in G(N, m)$ with $\operatorname{dim}\left(P_{1} \cap P_{2}\right)=m-1$, we have that for every $v \in \mathbb{R}^{N}$

$$
\Varangle\left(P_{1}, P_{2}\right)=\left|\pi_{P_{1}}(v)-\pi_{P_{2}}(v)\right|
$$

is equivalent to

$$
v \in \mathbb{S}^{N-1} \cap\left(P_{1} \cap P_{2}\right)^{\perp} \cap \operatorname{span}\left(P_{1}, P_{2}\right) .
$$

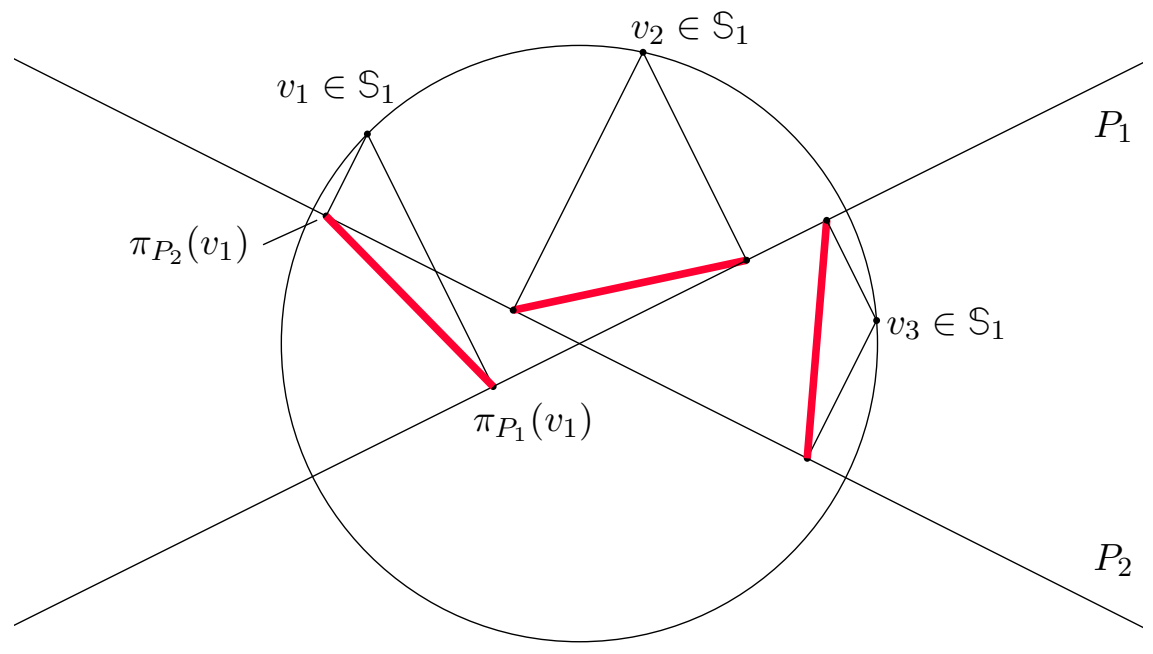

Figure 2.3: For every $v \in \mathbb{S}^{1}$, we get $\Varangle\left(P_{1}, P_{2}\right)=\left|\pi_{P_{1}}(v)-\pi_{P_{2}}(v)\right|$. (All red segments have the same length.) 
Proof. The first direction follows immediately from Lemma 2.22

Now let $v \in \mathbb{S}^{N-1} \cap\left(P_{1} \cap P_{2}\right)^{\perp} \cap \operatorname{span}\left(P_{1}, P_{2}\right)$. We choose an orthonormal basis of $P_{1} \cap P_{2}=$ $\operatorname{span}\left(o_{1}, \ldots, o_{m-1}\right)$ and extend this to orthonormal bases of $P_{1}$ and $P_{2}$

$$
\begin{aligned}
& P_{1}=\operatorname{span}\left(o_{1}, \ldots, o_{m-1}, X\right) \\
& P_{2}=\operatorname{span}\left(o_{1}, \ldots, o_{m-1}, Y\right) .
\end{aligned}
$$

The vector $v$ fulfils $\left\langle v, o_{i}\right\rangle=0$ for all $i \in\{1, \ldots, m-1\}$ because $v \in\left(P_{1} \cap P_{2}\right)^{\perp}$ and there exists $\alpha_{i} \in \mathbb{R}$ with $v=\sum_{i=1}^{m-1} \alpha_{i} o_{i}+\alpha_{m} X+\alpha_{m+1} Y$. This implies $v=\alpha_{m} X+\alpha_{m+1} Y$. The unit vectors $X$ and $Y$ are linearly independent and we are able to define $\bar{Y}:=\frac{X-\langle X, Y\rangle Y}{|X-\langle X, Y\rangle Y|} \neq 0$. So $(Y, \bar{Y})$ is an orthonormal basis of $\operatorname{span}(X, Y)$ and because $|v|=1$, we find $c, d \in \mathbb{R}$ with $c^{2}+d^{2}=1$ and $v=c Y+d \bar{Y}$. We have

$$
\begin{aligned}
\pi_{P_{1}}(Y) & =\langle X, Y\rangle X, \\
\pi_{P_{2}}(X) & =\langle X, Y\rangle Y, \\
|X-\langle X, Y\rangle Y|^{2} & =\langle X-\langle X, Y\rangle Y, X-\langle X, Y\rangle Y\rangle \\
& =\langle X, X\rangle-2\langle X,\langle X, Y\rangle Y\rangle+\langle\langle X, Y\rangle Y,\langle X, Y\rangle Y\rangle \\
& =1-2\langle X, Y\rangle^{2}+\langle X, Y\rangle^{2}\langle Y, Y\rangle \\
& =1-\langle X, Y\rangle^{2} .
\end{aligned}
$$

Now, using linearity of the projection, we obtain

$$
\pi_{P_{1}}(\bar{Y})=\pi_{P_{1}}\left(\frac{X-\langle X, Y\rangle Y}{|X-\langle X, Y\rangle Y|}\right)=\frac{X-\langle X, Y\rangle\langle X, Y\rangle X}{\sqrt{1-\langle X, Y\rangle^{2}}}=X \sqrt{1-\langle X, Y\rangle^{2}} .
$$

and

$$
\pi_{P_{2}}(\bar{Y})=\pi_{P_{2}}\left(\frac{X-\langle X, Y\rangle Y}{|X-\langle X, Y\rangle Y|}\right)=\frac{\langle X, Y\rangle Y-\langle X, Y\rangle Y}{\sqrt{1-\langle X, Y\rangle^{2}}}=0 .
$$

With this identities we get

$$
\begin{aligned}
& \left|\pi_{P_{1}}(v)-\pi_{P_{2}}(v)\right|^{2} \\
= & \left|c \pi_{P_{1}}(Y)+d \pi_{P_{1}}(\bar{Y})-(c \underbrace{\pi_{P_{2}}(Y)}_{=Y}+d \underbrace{\pi_{P_{2}}(\bar{Y})}_{=0})\right|^{2} \\
= & \left|c X\langle X, Y\rangle+d X \sqrt{1-\langle X, Y\rangle^{2}}-c Y\right|^{2} \\
= & \left\langle c X\langle X, Y\rangle+d X \sqrt{1-\langle X, Y\rangle^{2}}-c Y, c X\langle X, Y\rangle+d X \sqrt{1-\langle X, Y\rangle^{2}}-c Y\right\rangle \\
= & \langle c X\langle X, Y\rangle, c X\langle X, Y\rangle\rangle+2\left\langle c X\langle X, Y\rangle, d X \sqrt{1-\langle X, Y\rangle^{2}}\right\rangle-2\langle c X\langle X, Y\rangle, c Y\rangle \\
& +\left\langle d X \sqrt{1-\langle X, Y\rangle^{2}}, d X \sqrt{1-\langle X, Y\rangle^{2}}\right\rangle+\langle c Y, c Y\rangle-2\left\langle d X \sqrt{1-\langle X, Y\rangle^{2}}, c Y\right\rangle \\
= & c^{2}\langle X, Y\rangle^{2}+d^{2}\left(1-\langle X, Y\rangle^{2}\right)+c^{2} \\
& +2 c d\langle X, Y\rangle \sqrt{1-\langle X, Y\rangle^{2}}-2 c^{2}\langle X, Y\rangle^{2}-2 c d \sqrt{1-\langle X, Y\rangle^{2}}\langle X, Y\rangle \\
= & -c^{2}\langle X, Y\rangle^{2}-d^{2}\langle X, Y\rangle^{2}+c^{2}+d^{2} \\
= & -\langle X, Y\rangle^{2}+1
\end{aligned}
$$


so that

$$
\left|\pi_{P_{1}}(v)-\pi_{P_{2}}(v)\right|=\sqrt{1-\langle X, Y\rangle^{2}}
$$

is independent of $v \in \mathbb{S}^{N-1} \cap\left(P_{1} \cap P_{2}\right)^{\perp} \cap \operatorname{span}\left(P_{1}, P_{2}\right)$. The definition of the angle yields

$$
\Varangle\left(P_{1}, P_{2}\right)=\sup _{w \in \mathbb{S}^{N-1}}\left|\pi_{P_{1}}(w)-\pi_{P_{2}}(w)\right|
$$

and so there exists some $\hat{w} \in \mathbb{S}^{N-1}$ with

$$
\Varangle\left(P_{1}, P_{2}\right)=\left|\pi_{P_{1}}(w)-\pi_{P_{2}}(w)\right| .
$$

Using the first direction, we get $\hat{w} \in \mathbb{S}^{N-1} \cap\left(P_{1} \cap P_{2}\right)^{\perp} \cap \operatorname{span}\left(P_{1}, P_{2}\right)$ and so we obtain

$$
\begin{aligned}
\Varangle\left(P_{1}, P_{2}\right) & =\left|\pi_{P_{1}}(\hat{w})-\pi_{P_{2}}(\hat{w})\right| \\
& =\sqrt{1-\langle X, Y\rangle^{2}} \\
& =\left|\pi_{P_{1}}(v)-\pi_{P_{2}}(v)\right| .
\end{aligned}
$$

Lemma 2.31. Under the conditions of Corollary 2.5, i.e., $P_{1}, P_{2} \in \mathcal{P}(N, m)$ with $\operatorname{dim} P_{1}=$ $\operatorname{dim} P_{2}=m<N, \operatorname{dim}\left(P_{1} \cap P_{2}\right)=m-1$ and $a_{1} \in P_{1} \backslash P_{2}$, we have

$$
\frac{\left|a_{1}-\pi_{P_{2}}\left(a_{1}\right)\right|}{\left|a_{1}-\pi_{P_{1} \cap P_{2}}\left(a_{1}\right)\right|}=\Varangle\left(P_{1}, P_{2}\right) .
$$

Proof. In the proof of Corollary 2.5, we find $U, V \in G(N, m)$ and $u_{1} \in U \backslash V$ with

$$
\frac{\left|a_{1}-\pi_{P_{2}}\left(a_{1}\right)\right|}{\left|a_{1}-\pi_{P_{1} \cap P_{2}}\left(a_{1}\right)\right|}=\frac{\left|u_{1}-\pi_{V}\left(u_{1}\right)\right|}{\left|u_{1}-\pi_{U \cap V}\left(u_{1}\right)\right|} .
$$

In the proof of Lemma 2.4, we find $o_{U} \in \mathbb{S}^{N-1} \cap(U \cap V)^{\perp} \cap \operatorname{span}(U, V)$ with

$$
\frac{\left|u_{1}-\pi_{V}\left(u_{1}\right)\right|}{\left|u_{1}-\pi_{U \cap V}\left(u_{1}\right)\right|}=\left|o_{U}-\pi_{V}\left(o_{U}\right)\right|=\left|\pi_{U}\left(o_{U}\right)-\pi_{V}\left(o_{U}\right)\right| .
$$

Using Lemma 2.30, we obtain altogether

$$
\frac{\left|a_{1}-\pi_{P_{2}}\left(a_{1}\right)\right|}{\left|a_{1}-\pi_{P_{1} \cap P_{2}}\left(a_{1}\right)\right|}=\left|\pi_{U}\left(o_{U}\right)-\pi_{V}\left(o_{U}\right)\right|=\Varangle(U, V)=\Varangle\left(P_{1}, P_{2}\right) .
$$





\section{Integral Menger curvature and rectifiability}

\subsection{Main result}

Let $n, N \in \mathbb{N}$ with $1 \leq n<N$. We start with some definitions necessary for our main result. Definition 3.1 (Proper integrand). Let $\mathcal{K}:\left(\mathbb{R}^{N}\right)^{n+2} \rightarrow[0, \infty)$ and $p>1$. We say that $\mathcal{K}^{p}$ is a proper integrand if it fulfils the following four conditions:

- $\mathcal{K}$ is $\left(\mathcal{H}^{n}\right)^{n+2}$-measurable, where $\left(\mathcal{H}^{n}\right)^{n+2}$ denotes the $n+2$-times product measure of $\mathcal{H}^{n}$.

- There exists some constants $c=c(n, \mathcal{K}, p) \geq 1$ and $l=l(n, \mathcal{K}, p) \geq 1$ so that, for all $t>0, C \geq 1, x \in \mathbb{R}^{N}$ and all $\left(n, \frac{t}{C}\right)$-simplices $\Delta\left(x_{0}, \ldots, x_{n}\right) \subset B(x, C t)$, we have

$$
\left(\frac{d\left(w, \operatorname{aff}\left(x_{0}, \ldots, x_{n}\right)\right)}{t}\right)^{p} \leq c C^{l} t^{n(n+1)} \mathcal{K}^{p}\left(x_{0}, \ldots, x_{n}, w\right)
$$

for all $w \in B(x, C t)$.

- For all $t>0$, we have

$$
t^{n(n+1)} \mathcal{K}^{p}\left(t x_{0}, \ldots, t x_{n+1}\right)=\mathcal{K}^{p}\left(x_{0}, \ldots, x_{n+1}\right) .
$$

- $\mathcal{K}$ is invariant under every translation, i.e., for every $b \in \mathbb{R}$, we have

$$
\mathcal{K}\left(x_{0}+b, \ldots, x_{n+1}+b\right)=\mathcal{K}\left(x_{0}, \ldots, x_{n+1}\right) .
$$

Definition 3.2. (i) We name a Borel set $E \subset \mathbb{R}^{N}$ purely $n$-unrectifiable if for every Lipschitz continuous function $\gamma: \mathbb{R}^{n} \rightarrow \mathbb{R}^{N}$, we have

$$
\mathcal{H}^{n}\left(E \cap \gamma\left(\mathbb{R}^{n}\right)\right)=0 .
$$

(ii) We name a Borel set $E \subset \mathbb{R}^{N} n$-rectifiable if there exists some countable family of Lipschitz continuous functions $\gamma_{i}: \mathbb{R}^{n} \rightarrow \mathbb{R}^{N}$ so that

$$
\mathcal{H}^{n}\left(E \backslash \bigcup_{i=1}^{\infty} \gamma_{i}\left(\mathbb{R}^{n}\right)\right)=0
$$

Definition 3.3 (integral Menger curvature). Let $E \subset \mathbb{R}^{N}$ be a Borel set. We define the integral Menger curvature of $E$ with integrand $\mathcal{K}^{p}$ by

$$
\mathcal{M}_{\mathcal{K}^{p}}(E):=\int_{E} \ldots \int_{E} \mathcal{K}^{p}\left(x_{0}, \ldots, x_{n+1}\right) \mathrm{d} \mathcal{H}^{n}\left(x_{0}\right) \ldots \mathrm{d} \mathcal{H}^{n}\left(x_{n+1}\right) .
$$

Now we can state our main result.

Theorem 3.4. Let $E \subset \mathbb{R}^{N}$ be a borel set with $\mathcal{M}_{\mathcal{K}^{2}}(E)<\infty$, where $\mathcal{K}^{2}$ is some proper integrand. Then $E$ is n-rectifiable. 


\subsection{Examples of admissible integrands}

In the following, we give some examples of integrands, which fulfil the conditions of a proper integrand, known from several papers working with integral Menger curvatures.

We start with the definition of some set $X_{0} \subset\left(\mathbb{R}^{N}\right)^{n+2}$ where we collect all flat simplices. This means, we collect all $n+2$-tuples of points in $\mathbb{R}^{N}$ so that the elements of such a tuple are laying in some $n$-dimensional plane where this plane can change from tuple to tuple. It is plausible to give some integrand the value 0 on $X_{0}$.

Definition 3.5. We define the set

$$
X_{0}:=\left\{\left(x_{0}, \ldots, x_{n+1}\right) \in\left(\mathbb{R}^{N}\right)^{n+2} \mid \operatorname{Gram}\left(x_{1}-x_{0}, \ldots, x_{n+1}-x_{0}\right)=0\right\}
$$

(the Gram determinant is defined in Definition 2.11) which is the set of all simplices with $n+2$ vertices in $\mathbb{R}^{N}$ which span at most an $n$-dimensional affine subspace. The set $X_{0}$ is the fibre of 0 under a continuous function, so $X_{0}$ is a closed subset of $\left(\mathbb{R}^{N}\right)^{n+2}$, which implies that $X_{0}$ is $\left(\mathcal{H}^{n}\right)^{n+2}$-measurable (as a product of Borel measures $\left(\mathcal{H}^{n}\right)^{n+2}$ is a Borel measure as well, see Lemma A.11).

The following Lemma is helpful to prove that a given integrand fulfils the second condition of a proper integrand.

Lemma 3.6. Let $t>0, C \geq 1, x \in \mathbb{R}^{N}, w \in B(x, C t)$ and let $S=\Delta\left(x_{0}, \ldots, x_{n}\right) \subset B(x, C t)$ be some $\left(n, \frac{t}{C}\right)$-simplex. Setting $S_{w}=\Delta\left(x_{0}, \ldots, x_{n}, w\right)$ and choosing $i, j \in\{0, \ldots, n\}$ with $j \neq i$ we have the following statements:

- $\frac{t}{C} \leq d\left(x_{i}, x_{j}\right) \leq \operatorname{diam}\left(S_{w}\right) \leq 2 C t$,

- $d\left(x_{i}, w\right) \leq 2 C t$,

- $\frac{t^{n}}{C^{n} n !} \leq \mathcal{H}^{n}(S) \leq \frac{(2 C)^{n}}{n !} t^{n}$

- $\mathcal{H}^{n}(S) \leq A\left(S_{w}\right) \leq\left[(n+1) 2 C^{2}+1\right] \mathcal{H}^{n}(S)$,

- $d\left(w, \operatorname{aff}\left(x_{0}, \ldots, x_{n}\right)\right)=n \frac{\mathcal{H}^{n+1}\left(S_{w}\right)}{\mathcal{H}^{n}(S)}$,

where $A\left(S_{w}\right)$ denotes the surface area of the simplex $S_{w}$.

Proof. Since $S$ is an $\left(n, \frac{t}{C}\right)$-simplex, we have

$$
\frac{t}{C} \leq \mathfrak{h}_{i}(S) \leq d\left(x_{i}, x_{j}\right) \leq \operatorname{diam}\left(S_{w}\right)=\max _{\substack{l, m \in \\\{0, \ldots, n\}}}\left\{d\left(x_{l}, x_{m}\right), d\left(x_{l}, w\right)\right\} \leq 2 C t
$$

and because of $x_{i}, w \in B(x, C t)$, we get

$$
d\left(x_{i}, w\right) \leq 2 C t .
$$

Now, with Remark 2.12, we conclude that $\mathcal{H}^{n}(S)=\frac{1}{n !} \prod_{l=0}^{n-1} d\left(x_{l}, \operatorname{aff}\left(x_{l+1}, \ldots, x_{n}\right)\right)$ which implies with Remark 2.10

$$
\left.\frac{t^{n}}{C^{n} n !} \stackrel{(3.1)}{\leq} \frac{1}{n !} \prod_{l=0}^{n-1} \mathfrak{h}_{l}(S) \leq \mathcal{H}^{n}(S) \leq \frac{1}{n !} \prod_{l=0}^{n-1} d\left(x_{l}, x_{n}\right)\right) \stackrel{(3.1)}{\leq} \frac{(2 C)^{n}}{n !} t^{n} .
$$


Using Remark 2.12, we obtain

$$
\begin{aligned}
\mathcal{H}^{n}\left(\mathfrak{f c}_{i}\left(S_{w}\right)\right) & \stackrel{2.12}{=} \frac{1}{n} \underbrace{\mathfrak{h}_{w}\left(\mathfrak{f}_{i}\left(S_{w}\right)\right)}_{\leq d\left(w, x_{j}\right) \leq 2 C t} \mathcal{H}^{n-1}\left(\mathfrak{f}_{i, w}\left(S_{w}\right)\right) \\
& \leq \frac{1}{n} 2 C^{2} \frac{t}{C} \mathcal{H}^{n-1}\left(\mathfrak{f}_{i}(S)\right) \\
& \stackrel{(3.1)}{\leq} \frac{1}{n} 2 C^{2} \mathfrak{h}_{i}(S) \mathcal{H}^{n-1}\left(\mathfrak{f c}_{i}(S)\right) \\
& \stackrel{2.12}{=} 2 C^{2} \mathcal{H}^{n}(S),
\end{aligned}
$$

so that with $A\left(S_{w}\right)=\sum_{i=0}^{n} \mathcal{H}^{n}\left(\mathfrak{f}_{i} S_{w}\right)+\mathcal{H}^{n}\left(\mathfrak{f}_{w} S_{w}\right)$ and $\mathfrak{f c}_{w}\left(S_{w}\right)=S$, we get

$$
\mathcal{H}^{n}(S) \leq A\left(S_{w}\right) \leq\left[(n+1) 2 C^{2}+1\right] \mathcal{H}^{n}(S) .
$$

Finally, with Remark 2.12 and using that $S=\mathfrak{f} \mathfrak{c}_{w}\left(S_{w}\right)$, we deduce

$$
d\left(w, \operatorname{aff}\left(x_{0}, \ldots, x_{n}\right)\right)=\mathfrak{h}_{w}\left(S_{w}\right)=\frac{\mathfrak{h}_{w}\left(S_{w}\right) \cdot \mathcal{H}^{n}\left(\mathfrak{f}_{w}\left(S_{w}\right)\right)}{\mathcal{H}^{n}(S)}=\frac{n \mathcal{H}^{n+1}\left(S_{w}\right)}{\mathcal{H}^{n}(S)} .
$$

\section{Proper Integrands with exponent 2}

Now we can state some examples of proper integrands. We mention that for our main statement we can only use those integrands which are proper for integrability exponent $p=2$. We start with the one used in the introduction of this work.

Lemma 3.7. Let $x_{0}, \ldots, x_{n+1} \in \mathbb{R}^{N}$ and set

$$
\mathcal{K}\left(x_{0}, \ldots, x_{n+1}\right):= \begin{cases}\frac{\mathcal{H}^{n+1}\left(\Delta\left(x_{0}, \ldots, x_{n+1}\right)\right)}{\Pi_{0 \leq i<j \leq n+1} d\left(x_{i}, x_{j}\right)} & \left(x_{0}, \ldots, x_{n+1}\right) \in\left(\mathbb{R}^{N}\right)^{n+2} \backslash X_{0}, \\ 0 & \left(x_{0}, \ldots, x_{n+1}\right) \in X_{0},\end{cases}
$$

where $X_{0}$ is the set of flat $(n+1)$-simplices, defined in Definition 3.5. Then $\mathcal{K}^{p}$ is a proper integrand with $p=2$.

Proof. The sets $\left(\mathbb{R}^{N}\right)^{n+2} \backslash X_{0}$ and $X_{0}$ are $\left(\mathcal{H}^{n}\right)^{n+2}$-measurable and the function $\mathcal{K}$ is continuous on $\left(\mathbb{R}^{N}\right)^{n+2} \backslash X_{0}$ as a composition of continuous functions, which implies that $\mathcal{K}$ is an $\left(\mathcal{H}^{n}\right)^{n+2}$ measurable function on $\left(\mathbb{R}^{N}\right)^{n+2}$.

Now we focus on the second and third condition. Let $t>0, C \geq 1, x \in \mathbb{R}^{N}, \Delta\left(x_{0}, \ldots, x_{n}\right) \subset$ $B(x, C t)$ be an $\left(n, \frac{t}{C}\right)$-simplex and $w \in B(x, C t)$. Using Lemma 3.6 and $p=2$, there exists some generic constant $\hat{C}=\hat{C}(n, C)$ so that

$$
\begin{aligned}
\left(\frac{d\left(w, \operatorname{aff}\left(x_{0}, \ldots, x_{n+1}\right)\right)}{t}\right)^{p} & \leq \hat{C}\left(\frac{\mathcal{H}^{n+1}\left(\Delta\left(x_{0}, \ldots, x_{n}, w\right)\right)}{t \cdot t^{n}}\right)^{2} \\
& \leq \hat{C} t^{n(n+1)}\left(\frac{\mathcal{H}^{n+1}\left(\Delta\left(x_{0}, \ldots, x_{n}, w\right)\right)}{t^{\frac{1}{2}(n+1)(n+2)}}\right)^{2} \\
& \leq \hat{C} t^{n(n+1)} \mathcal{K}_{n}^{2}\left(x_{0}, \ldots, x_{n+1}\right) .
\end{aligned}
$$


We get for some $t>0$ and some simplex $T=\Delta\left(x_{0}, \ldots, x_{n+1}\right)$ that

$$
t^{n(n+1)} \mathcal{K}^{p}(t T)=t^{n(n+1)} \frac{t^{2(n+1)}}{t^{(n+1)(n+2)}} \mathcal{K}^{2}(T)=\mathcal{K}^{p}(T) .
$$

The fourth condition follows directly from the definition of $\mathcal{K}$.

The next integrand is used by Lerman and Whitehouse in [LW11, LW09].

Lemma 3.8. Let $x_{0}, \ldots, x_{n+1} \in \mathbb{R}^{N}$ and set

$$
c_{n}^{2}\left(x_{0}, \ldots, x_{n+1}\right):=\frac{1}{n+2} \cdot \frac{\operatorname{Vol}_{n+1}\left(\Delta\left(x_{0}, \ldots, x_{n+1}\right)\right)^{2}}{\operatorname{diam}\left(\Delta\left(x_{0}, \ldots, x_{n+1}\right)\right)^{n(n+1)}} \sum_{i=0}^{n+1} \frac{1}{\prod_{\substack{j=0 \\ j \neq i}}^{n+1}\left|x_{j}-x_{i}\right|^{2}}
$$

and

$$
\mathcal{K}\left(x_{0}, \ldots, x_{n+1}\right):= \begin{cases}c_{n}\left(x_{0}, \ldots, x_{n+1}\right) & \left(x_{0}, \ldots, x_{n+1}\right) \in\left(\mathbb{R}^{N}\right)^{n+2} \backslash X_{0}, \\ 0 & \left(x_{0}, \ldots, x_{n+1}\right) \in X_{0},\end{cases}
$$

where $\operatorname{Vol}_{n+1}$ is $(n+1)$ ! times the volume of the simplex $\Delta\left(x_{0}, \ldots, x_{n+1}\right)$, which is equal to the volume of the parallelotope spanned by this simplex, cf. Definition 2.11. Furthermore, $X_{0}$ is the set of flat $(n+1)$-simplices defined in Definition 3.5. Then $\mathcal{K}^{p}$ is a proper integrand with $p=2$.

Proof. The same argumentation as in Lemma 3.7, implies that $\mathcal{K}$ is an $\left(\mathcal{H}^{n}\right)^{n+2}$-measurable function.

Let $t>0, C \geq 1, x \in \mathbb{R}^{N}, \Delta\left(x_{0}, \ldots, x_{n}\right) \subset B(x, C t)$ be an $\left(n, \frac{t}{C}\right)$-simplex and $w \in B(x, C t)$. Using Lemma 3.6 and $p=2$, there exists some generic constant $\hat{C}=\hat{C}(n, C)$ so that

$$
\begin{aligned}
\left(\frac{d\left(w, \operatorname{aff}\left(x_{0}, \ldots, x_{n+1}\right)\right)}{t}\right)^{p} & \leq \hat{C}\left(\frac{\operatorname{Vol}_{n+1}\left(\Delta\left(x_{0}, \ldots, x_{n}, w\right)\right)}{t \cdot t^{n}}\right)^{2} \\
& \leq \hat{C} t^{n(n+1)} \frac{\operatorname{Vol}_{n+1}\left(\Delta\left(x_{0}, \ldots, x_{n}, w\right)\right)^{2}}{t^{n(n+1)}} \sum_{i=0}^{n+1} \frac{1}{t^{2(n+1)}} \\
& \leq \hat{C} t^{n(n+1)} c_{n}^{2}\left(x_{0}, \ldots, x_{n+1}\right) .
\end{aligned}
$$

We get for some $t>0$ and some simplex $T=\Delta\left(x_{0}, \ldots, x_{n+1}\right)$ that

$$
t^{n(n+1)} \mathcal{K}^{p}(t T)=t^{n(n+1)}\left(\frac{t^{2(n+1)}}{t^{n(n+1)}} \frac{1}{t^{2(n+1)}} c_{n}^{2}(T)\right)=\mathcal{K}^{p}(T) .
$$

The following integrand is mentioned among others in [LW09, section 6]. It can be regarded as an adaptation of the integrand from the following Lemma 3.11 used e.g. in [BK12, KSvdM13]. 
Lemma 3.9. Let $x_{0}, \ldots, x_{n+1} \in \mathbb{R}^{N}$ and set

$$
\mathcal{K}\left(x_{0}, \ldots, x_{n+1}\right):= \begin{cases}\frac{\mathcal{H}^{n+1}\left(\Delta\left(x_{0}, \ldots, x_{n+1}\right)\right)}{\operatorname{diam} \Delta\left(x_{0}, \ldots, x_{n+1} \frac{(n+1)(n+2)}{2}\right.} & \left(x_{0}, \ldots, x_{n+1}\right) \in\left(\mathbb{R}^{N}\right)^{n+2} \backslash X_{0}, \\ 0 & \left(x_{0}, \ldots, x_{n+1}\right) \in X_{0},\end{cases}
$$

where $X_{0}$ is the set of flat $(n+1)$-simplices defined in Definition 3.5. Then $\mathcal{K}^{p}$ is a proper integrand with $p=2$.

Proof. The proof is completely analogue to the preceding ones.

\section{Proper Integrands with exponents different from 2}

Now we present some integrands for integral Menger curvature used in several papers, where the scaling behaviour implies that our main result can not be applied. Nevertheless, most of our partial results are valid for these integrands.

The first integrand we consider was introduced for $n=2, N=3$ in [SvdM11].

Lemma 3.10. Let $x_{0}, \ldots, x_{n+1} \in \mathbb{R}^{N}$ and set

$$
\mathcal{K}\left(x_{0}, \ldots, x_{n+1}\right):= \begin{cases}\frac{V(T)}{A(T)(\operatorname{diam} T)^{2}} & \left(x_{0}, \ldots, x_{n+1}\right) \in\left(\mathbb{R}^{N}\right)^{n+2} \backslash X_{0}, \\ 0 & \left(x_{0}, \ldots, x_{n+1}\right) \in X_{0},\end{cases}
$$

where $V(T)$ is the volume of the simplex $T=\Delta\left(x_{0}, \ldots, x_{n+1}\right), A(T)$ is the surface area of $T$ and $X_{0}$ is the set of flat $(n+1)$-simplices defined in Definition 3.5. Then $\mathcal{K}^{p}$ is a proper integrand with $p=n(n+1)$.

Proof. The same argumentation as in Lemma 3.7, implies that $\mathcal{K}$ is an $\left(\mathcal{H}^{n}\right)^{n+2}$-measurable function.

Let $t>0, C \geq 1, x \in \mathbb{R}^{N}, S=\Delta\left(x_{0}, \ldots, x_{n}\right) \subset B(x, C t)$ be an $\left(n, \frac{t}{C}\right)$-simplex and $w \in B(x, C t)$. We set $S_{w}=\Delta\left(x_{0}, \ldots, x_{n}, w\right)$ and using Lemma 3.6, we obtain

$$
\begin{aligned}
\frac{d\left(w, \operatorname{aff}\left(x_{0}, \ldots, x_{n}\right)\right)}{t} & =t \frac{n \mathcal{H}^{n+1}\left(S_{w}\right)}{\mathcal{H}^{n}(S) \cdot t^{2}} \\
& =t\left[(n+1) 2 C^{2}+1\right](2 C)^{2} \frac{n \cdot \mathcal{H}^{n+1}\left(S_{w}\right)}{A\left(S_{w}\right) \cdot\left(\operatorname{diam}\left(S_{w}\right)\right)^{2}} \\
& <\operatorname{tn}(n+2) 8 C^{4} \mathcal{K}\left(x_{0}, \ldots, x_{n}, w\right) .
\end{aligned}
$$

Hence we get with $p=n(n+1)$

$$
\left(\frac{d\left(w, \operatorname{aff}\left(x_{0}, \ldots, x_{n}\right)\right)}{t}\right)^{p} \leq(n(n+2) 8)^{p} C^{4 p} t^{n(n+1)} \mathcal{K}^{p}\left(x_{0}, \ldots, x_{n}, w\right) .
$$

For some $t>0$ and some simplex $T=\Delta\left(x_{0}, \ldots, x_{n+1}\right)$ we get, because of the scaling behaviour of the volume $V(T)$, the surface area $A(T)$ and the diameter $\operatorname{diam}(T)$

$$
t^{n(n+1)} \mathcal{K}^{p}(t T)=t^{n(n+1)}\left(\frac{t^{n+1} V(T)}{t^{n} A(T) t^{2} \operatorname{diam}(T)^{2}}\right)^{n(n+1)}=\mathcal{K}^{p}(T) .
$$


The fourth condition follows directly from the definition of $\mathcal{K}$. All in all, we have verified all four conditions of a proper integrand.

The next integrand is used, for example, in [BK12, KSvdM13].

Lemma 3.11. Let $x_{0}, \ldots, x_{n+1} \in \mathbb{R}^{N}$ and set

$$
\mathcal{K}\left(x_{0}, \ldots, x_{n+1}\right):= \begin{cases}\frac{\mathcal{H}^{n+1}\left(\Delta\left(x_{0}, \ldots, x_{n+1}\right)\right)}{\operatorname{diam}\left(\Delta\left(x_{0}, \ldots, x_{n+1}\right)\right)^{n+2}} & \left(x_{0}, \ldots, x_{n+1}\right) \in\left(\mathbb{R}^{N}\right)^{n+2} \backslash X_{0}, \\ 0 & \left(x_{0}, \ldots, x_{n+1}\right) \in X_{0},\end{cases}
$$

where $X_{0}$ is the set of flat $(n+1)$-simplices defined in Definition 3.5. Then $\mathcal{K}^{p}$ is a proper integrand with $p=n(n+1)$.

Proof. The same argumentation as in Lemma 3.7, implies that $\mathcal{K}$ is an $\left(\mathcal{H}^{n}\right)^{n+2}$-measurable function.

Let $t>0, C \geq 1, x \in \mathbb{R}^{N}, \Delta\left(x_{0}, \ldots, x_{n}\right) \subset B(x, C t)$ be an $\left(n, \frac{t}{C}\right)$-simplex and $w \in B(x, C t)$. We set $S_{w}=\Delta\left(x_{0}, \ldots, x_{n+1}, w\right)$ and using Lemma 3.6 and $p=n(n+1)$, we obtain

$$
\begin{aligned}
\left(\frac{d\left(w, \operatorname{aff}\left(x_{0}, \ldots, x_{n}\right)\right)}{t}\right)^{p} & =t^{n(n+1)}\left(\frac{n \mathcal{H}^{n+1}\left(S_{w}\right)}{\mathcal{H}^{n}(S) \cdot t^{2}}\right)^{p} \\
& \leq 2^{(n+2) p} C^{(2 n+2) p} n ! n t^{n(n+1)} \mathcal{K}^{p}\left(x_{0}, \ldots, x_{n}, w\right)
\end{aligned}
$$

and with

$$
t^{n(n+1)} \mathcal{K}^{p}(t T)=t^{n(n+1)}\left(\frac{t^{n+1} \mathcal{H}^{n+2}(T)}{t^{n+2} \operatorname{diam}(T)^{n+2}}\right)^{n(n+1)}=\mathcal{K}^{p}(T)
$$

the first three conditions of a proper integrand are fulfilled. The fourth follows directly from the definition of $\mathcal{K}$.

Finally, Léger suggested the following integrand in [Lég99] for a higher dimensional analogue of his theorem. Unfortunately, we can not affirm his suggestion. For $n=1$ up to a factor of 2 , this integrand gives the inverse of the circumcircle of the three points $x_{0}, x_{1}, x_{2}$, hence the original integrand of Léger.

Lemma 3.12. Let $x_{0}, \ldots, x_{n+1} \in \mathbb{R}^{N}$ and set

$$
\mathcal{K}\left(x_{0}, \ldots, x_{n+1}\right):= \begin{cases}\frac{d\left(x_{n+1}, \operatorname{aff}\left(x_{0}, \ldots, x_{n}\right)\right)}{d\left(x_{n+1}, x_{0}\right) \ldots d\left(x_{n+1}, x_{n}\right)} & \left(x_{0}, \ldots, x_{n+1}\right) \in\left(\mathbb{R}^{N}\right)^{n+2} \backslash X_{0}, \\ 0 & \left(x_{0}, \ldots, x_{n+1}\right) \in X_{0},\end{cases}
$$

where $X_{0}$ is the set of flat $(n+1)$-simplices defined in Definition 3.5. Then $\mathcal{K}^{p}$ is a proper integrand with $p=(n+1)$. 
Proof. The same argumentation as in Lemma 3.7, implies that $\mathcal{K}$ is an $\left(\mathcal{H}^{n}\right)^{n+2}$-measurable function.

Let $t>0, C \geq 1, x \in \mathbb{R}^{N}, \Delta\left(x_{0}, \ldots, x_{n}\right) \subset B(x, C t)$ be an $\left(n, \frac{t}{C}\right)$-simplex and $w \in B(x, C t)$. Using Lemma 3.6 and $p=n+1$, we obtain

$$
\begin{aligned}
\left(\frac{d\left(w, \operatorname{aff}\left(x_{0}, \ldots, x_{n+1}\right)\right)}{t}\right)^{p} & =(2 C)^{(n+1) p} t^{n p}\left(\frac{d\left(w, \operatorname{aff}\left(x_{0}, \ldots, x_{n+1}\right)\right)}{(2 C t)^{n+1}}\right)^{p} \\
& \leq(2 C)^{(n+1)(n+1)} t^{n(n+1)}\left(\frac{d\left(w, \operatorname{aff}\left(x_{0}, \ldots, x_{n+1}\right)\right)}{d\left(w, x_{0}\right) \ldots d\left(w, x_{n}\right)}\right)^{p} .
\end{aligned}
$$

Due to the scaling behaviour of the distance, we get for some $t>0$ and some simplex $T=\Delta\left(x_{0}, \ldots, x_{n+1}\right)$ that

$$
t^{n(n+1)} \mathcal{K}^{p}(t T)=t^{n(n+1)}\left(\frac{t d\left(x_{n+1}, \operatorname{aff}\left(x_{0}, \ldots, x_{n}\right)\right)}{t^{n+1} d\left(x_{n+1}, x_{0}\right) \ldots d\left(x_{n+1}, x_{n}\right)}\right)^{n+1}=\mathcal{K}^{p}(T) .
$$





\section{Proof of the main result}

At the end of this chapter (page 42), we will give a proof of our main result Theorem 3.4 under the assumption that the forthcoming Theorem 4.6 is correct. We start with a few lemmas helpful for this proof.

\subsection{Reduction to a symmetric integrand}

Lemma 4.1. Let $\mathcal{K}^{p}$ be some proper integrand (see Definition 3.1). There exists some proper integrand $\tilde{\mathcal{K}}^{p}$, which is symmetric in all components and fulfils $\mathcal{M}_{\mathcal{K}^{p}}(E)=\mathcal{M}_{\tilde{\mathcal{K}}^{p}}(E)$ for all Borel sets E.

Proof. We set

$$
\tilde{\mathcal{K}}^{p}\left(x_{0}, \ldots, x_{n+1}\right):=\frac{1}{\# S_{n+2}} \sum_{\phi \in S_{n+2}} \mathcal{K}^{p}\left(\phi\left(x_{0}, \ldots, x_{n+1}\right)\right),
$$

where $S_{n+2}$ is the symmetric group of all permutations of $n+2$ symbols. Obviously, $\tilde{\mathcal{K}}^{p}$ fulfils the first and the last condition of a proper integrand. Furthermore, we have

$$
\mathcal{K}^{p}\left(x_{0}, \ldots, x_{n}, w\right) \leq \# S_{n+2} \tilde{\mathcal{K}}^{p}\left(x_{0}, \ldots, x_{n+1}\right),
$$

which implies that $\tilde{\mathcal{K}}^{p}$ fulfils the second condition as well.

With Fubini's theorem [EG92, 1.4, Thm. 1], we obtain for some Borel set $E$

$$
\begin{aligned}
\mathcal{M}_{\tilde{\mathcal{K}}^{p}}(E) & =\frac{1}{\# S_{n+2}} \sum_{\phi \in S_{n+2}} \int_{E} \ldots \int_{E} \mathcal{K}^{p}\left(\phi\left(x_{0}, \ldots, x_{n+1}\right)\right) \mathrm{d} \mathcal{H}^{n}\left(x_{0}\right) \ldots \mathrm{d} \mathcal{H}^{n}\left(x_{n+1}\right) \\
& =\frac{1}{\# S_{n+2}} \sum_{\phi \in S_{n+2}} \int_{E} \ldots \int_{E} \mathcal{K}^{p}\left(z_{0}, \ldots, z_{n+1}\right) \mathrm{d} \mathcal{H}^{n}\left(z_{0}\right) \ldots \mathrm{d} \mathcal{H}^{n}\left(z_{n+1}\right) \\
& =\mathcal{M}_{\mathcal{K}^{p}}(E) .
\end{aligned}
$$

\subsection{Reduction to finite, compact and more regular sets with small curvature}

Lemma 4.2. Let $E$ be a Borel set with $\mathcal{M}_{\mathcal{K}^{p}}(E)<\infty$, where $\mathcal{K}^{p}$ is some proper integrand. Then we have $\mathcal{H}^{n}(E \cap B)<\infty$ for every ball $B$. 
Proof. Let $B$ be some ball and set $F:=E \cap B$. We prove the contraposition so we assume that $\mathcal{H}^{n}(F)=\infty$. With Lemma 2.17 , there exists some constant $C>0$ and some $(n+1,(n+3) C)$ simplex $T=\Delta\left(x_{0}, \ldots, x_{n+1}\right) \in B$ with $\mathcal{H}^{n}\left(B\left(x_{0}, C\right) \cap F\right)=\infty$ and $\mathcal{H}^{n}\left(B\left(x_{i}, C\right) \cap F\right)>0$ for all $i \in\{1, \ldots, n+1\}$. With Lemma 2.14 , we conclude that $S=\Delta\left(y_{0}, \ldots, y_{n+1}\right)$ is an $(n+1, C)$ simplex for all $y_{i} \in B\left(x_{i}, C\right), i \in\{0, \ldots, n+1\}$. For $t=C \sqrt{\frac{\operatorname{diam} B}{2 C}+1}$ and $\bar{C}=\sqrt{\frac{\operatorname{diam} B}{2 C}+1}$, we get $S \in B(x, t \bar{C})$, where $x$ is the centre of the ball $B$, and $S$ is an $\left(n+1, \frac{t}{C}\right)$-simplex. Hence we are in the right setting for using the second condition of a proper integrand. We obtain

$$
\begin{aligned}
& \mathcal{M}_{\mathcal{K}^{p}}(E) \\
= & \int_{E} \ldots \int_{E} \mathcal{K}^{p}\left(y_{0}, \ldots, y_{n+1}\right) \mathrm{d} \mathcal{H}^{n}\left(y_{0}\right) \ldots \mathrm{d} \mathcal{H}^{n}\left(y_{n+1}\right) \\
\geq & \int_{B\left(x_{n+1}, C\right) \cap F} \ldots \int_{B\left(x_{0}, C\right) \cap F} \mathcal{K}^{p}\left(y_{0}, \ldots, y_{n+1}\right) \mathrm{d} \mathcal{H}^{n}\left(y_{0}\right) \ldots \mathrm{d} \mathcal{H}^{n}\left(y_{n+1}\right) \\
\geq & C \int_{B\left(x_{n+1}, C\right) \cap F} \ldots \int_{B\left(x_{0}, C\right) \cap F} \frac{1}{t^{n(n+1)}}\left(\frac{d\left(y_{n+1}, \operatorname{aff}\left(y_{0}, \ldots, y_{n}\right)\right)}{t}\right)^{p} \mathrm{~d} \mathcal{H}^{n}\left(y_{0}\right) \ldots \mathrm{d} \mathcal{H}^{n}\left(y_{n+1}\right) \\
\geq & C(t, n, p) \int_{B\left(x_{n+1}, C\right) \cap F} \ldots \int_{B\left(x_{0}, C\right) \cap F} 1 \mathrm{~d} \mathcal{H}^{n}\left(y_{0}\right) \ldots \mathrm{d} \mathcal{H}^{n}\left(y_{n+1}\right) \\
\geq & C(t, n, p) \underbrace{\mathcal{H}^{n}\left(B\left(x_{n+1}, C\right) \cap F\right)}_{>0} \ldots \underbrace{\mathcal{H}^{n}\left(B\left(x_{1}, C\right) \cap F\right)}_{>0} \underbrace{\mathcal{H}^{n}\left(B\left(x_{0}, C\right) \cap F\right)}_{=\infty} \\
= & \infty .
\end{aligned}
$$

Lemma 4.3. In this lemma, the integrand $\mathcal{K}$ of $\mathcal{M}_{\mathcal{K}^{p}}$ only needs to be an $\left(\mathcal{H}^{n}\right)^{n+2}$-integrable function. Let $p>0, n<N$ and $E \subset \mathbb{R}^{N}$ be a Borel set with $0<\mathcal{H}^{n}(E)<\infty$ and $\mathcal{M}_{\mathcal{K}^{p}}(E)<\infty$. For all $\eta>0$, there exists some $E^{*} \subset E$ with

(i) $E^{*}$ is compact,

(ii) $\mathcal{M}_{\mathcal{K}^{p}}\left(E^{*}\right) \leq \eta\left(\operatorname{diam} E^{*}\right)^{n}$,

(iii) $\mathcal{H}^{n}\left(E^{*}\right)>\frac{\left(\operatorname{diam} E^{*}\right)^{n} \omega_{n}}{2^{2 n+2}}$,

(iv) $\forall x \in E^{*}, \forall t>0, \mathcal{H}^{n}\left(E^{*} \cap B(x, t)\right) \leq 2 \omega_{n} t^{n}$,

where $\omega_{n}=\mathcal{H}^{n}(B(0,1))$ is the $n$-dimensional volume of the $n$-dimensional unit ball.

Proof. Due to $0<\mathcal{H}^{n}(E)<\infty$ and [EG92, 2.3, Thm. 2], there exists a $\mathcal{H}^{n}$-nullset $M$ so that for all $x \in \tilde{E}:=E \backslash M$ we have

$$
\frac{1}{2^{n}} \leq \limsup _{t \rightarrow 0^{+}} \frac{\mathcal{H}^{n}(E \cap B(x, t))}{\omega_{n} t^{n}} \leq 1
$$


For $m \in \mathbb{N}$, we define

$$
\begin{aligned}
E_{m} & :=\left\{x \in E \mid \forall t \in\left(0, \frac{1}{m}\right), \mathcal{H}^{n}(E \cap B(x, t)) \leq 2 \omega_{n} t^{n}\right\} \\
& =\left\{x \in E \mid \sup _{t \in\left(0, \frac{1}{m}\right)} \frac{\mathcal{H}^{n}(E \cap B(x, t))}{t^{n}} \leq 2 \omega_{n}\right\} .
\end{aligned}
$$

At first, we show that $E_{m}$ is $\mathcal{H}^{n}$-measurable. We set $f_{t}(x):=\frac{\mathcal{H}^{n}(E \cap B(x, t))}{t^{n}}$ and conclude using Claim 2 of the proof of [EG92, 1.6.1, Thm 1] that $f_{t}$ is upper semicontinuous and thus Borel measurable. With [EG92, 1.1.2, Thm 6 (ii)], the function

$$
\sup _{t \in\left(0, \frac{1}{m}\right) \cap \mathbb{Q}} f_{t}(x)
$$

is also Borel measurable and so the set

$$
\hat{E}_{m}:=\left\{x \in E \mid \sup _{t \in\left(0, \frac{1}{m}\right) \cap \mathbb{Q}} \frac{\mathcal{H}^{n}(E \cap B(x, t))}{t^{n}} \leq 2 \omega_{n}\right\}
$$

is $\mathcal{H}^{n}$-measurable. There exists some sequence $\left(t_{i}\right)_{i \in \mathbb{N}}, t_{i} \in\left(0, \frac{1}{m}\right)$ with

$$
\lim _{i \rightarrow \infty} f_{t_{i}}(x)=\sup _{t \in\left(0, \frac{1}{m}\right)} f_{t}(x)
$$

For every $\varepsilon>0$ and $t_{i}$, there exists some $0 \leq s_{i}<\varepsilon$ with $t_{i} \leq t_{i}\left(1+s_{i}\right) \in \mathbb{Q} \cap\left(0, \frac{1}{m}\right)$, where $s_{i}:=0$ if $t_{i} \in \mathbb{Q}$. So we get

$$
\begin{aligned}
\sup _{t \in\left(0, \frac{1}{m}\right) \cap \mathbb{Q}} f_{t}(x) & \leq \sup _{t \in\left(0, \frac{1}{m}\right)} f_{t}(x) \\
& =\lim _{i \rightarrow \infty} f_{t_{i}}(x) \\
& \leq \lim _{i \rightarrow \infty} \frac{\mathcal{H}^{n}\left(E \cap B\left(x, t_{i}\left(1+s_{i}\right)\right)\right)}{t_{i}^{n}} \\
& =\lim _{i \rightarrow \infty}\left(1+s_{i}\right)^{n} f_{t_{i}\left(1+s_{i}\right)}(x) \\
& \leq(1+\varepsilon)^{n} \sup _{t \in\left(0, \frac{1}{m}\right) \cap \mathbb{Q}} f_{t}(x) .
\end{aligned}
$$

Since $\varepsilon$ was arbitrarily chosen, $E_{m}=\hat{E}_{m}$ is $\mathcal{H}^{n}$-measurable. We have

$$
\tilde{E} \subset \bigcup_{m \in \mathbb{N}} E_{m} \subset E
$$

because for $x \in \tilde{E}$ with (4.1) there exists some $m \in \mathbb{N}$ so that

$$
\mathcal{H}^{n}(E \cap B(x, t))<2 \omega_{n} t^{n} \quad \forall 0<t<\frac{1}{m}
$$


and we get $x \in E_{m}$. The second inclusion follows from the definition of $E_{m}$. By definition of $E_{m}$, we also get $E_{m} \subset E_{m+1} \subset E$ so that with [EG92, 1.1.1, Thm. 1]

$$
\mathcal{H}^{n}\left(\bigcup_{k=1}^{\infty} E_{k}\right)=\lim _{m \rightarrow \infty} \mathcal{H}^{n}\left(E_{m}\right)
$$

We have

$$
\mathcal{H}^{n}(E)=\mathcal{H}^{n}(\tilde{E} \cup M)=\mathcal{H}^{n}(\tilde{E}) \stackrel{(4.3)}{\leq} \mathcal{H}^{n}\left(\bigcup_{k=1}^{\infty} E_{k}\right) \stackrel{(4.3)}{\leq} \mathcal{H}^{n}(E)
$$

such that

$$
\lim _{m \rightarrow \infty} \mathcal{H}^{n}\left(E_{m}\right)=\mathcal{H}^{n}(E) .
$$

So there exists some $m \in \mathbb{N}$ with $\mathcal{H}^{n}\left(E_{m}\right) \geq \frac{1}{2} \mathcal{H}^{n}(E)$.

Since $E_{m} \subset E$, we conclude $\mathcal{M}_{\mathcal{K}^{p}}\left(E_{m}\right) \leq \mathcal{M}_{\mathcal{K}^{p}}(E)<\infty$.

Define for $\tau>0$

$$
\mathcal{I}(\tau):=\int_{A(\tau)} \mathcal{K}^{p}\left(x_{0}, \ldots, x_{n+1}\right) \mathrm{d} \mathcal{H}^{n}\left(x_{0}\right) \ldots \mathrm{d} \mathcal{H}^{n}\left(x_{n+1}\right),
$$

where $A(\tau):=\left\{\left(x_{0}, \ldots, x_{n+1}\right) \in E_{m}^{n+2} \mid d\left(x_{0}, x_{i}\right)<\tau\right.$ for all $\left.i \in\{1, \ldots, n+1\}\right\}$. We obtain for $x_{0} \in E_{m}$

$$
\begin{aligned}
A(\tau)_{x_{0}} & :=\left\{\left(x_{1}, \ldots, x_{n+1}\right) \in E_{m}^{n+1} \mid\left(x_{0}, \ldots, x_{n+1}\right) \in A(\tau)\right\} \\
& =\left\{\left(x_{1}, \ldots, x_{n+1}\right) \in E_{m}^{n+1} \mid d\left(x_{0}, x_{i}\right)<\tau \text { for all } i \in\{1, \ldots, n+1\}\right\} \\
& ={ }_{i=1}^{n+1}\left\{x \in E_{m} \mid d\left(x_{0}, x_{i}\right)<\tau\right\} \\
& =\left(B\left(x_{0}, \tau\right) \cap E_{m}\right)^{n+1}
\end{aligned}
$$

and, with Fubini's theorem [EG92, 1.4, Thm. 1] and for $0<\tau<\frac{1}{m}$, we can estimate

$$
\begin{aligned}
& \left(\mathcal{H}^{n}\right)^{n+2}(A(\tau)) \\
= & \int_{E_{m}}\left(\mathcal{H}^{n}\right)^{n+1}\left(A(\tau)_{x_{0}}\right) \mathrm{d} \mathcal{H}^{n}\left(x_{0}\right) \\
= & \int_{E_{m}}(\underbrace{\mathcal{H}^{n}\left(B\left(x_{0}, \tau\right) \cap E_{m}\right)}_{\leq 2 \omega_{n} \tau^{n}})^{n+1} \mathrm{~d} \mathcal{H}^{n}\left(x_{0}\right) \\
\stackrel{(4.2)}{\leq} & \left(2 \omega_{n} \tau^{n}\right)^{n+1} \int_{E_{m}} 1 \mathrm{~d} \mathcal{H}^{n}\left(x_{0}\right) \\
= & \left(2 \omega_{n} \tau^{n}\right)^{n+1} \mathcal{H}^{n}\left(E_{m}\right) \rightarrow 0 \quad \text { for } \tau \rightarrow 0
\end{aligned}
$$

such that

$$
\left(\mathcal{H}^{n}\right)^{n+2}(A(\tau)) \rightarrow 0 \quad \text { for } \tau \rightarrow 0 .
$$

With $\mathcal{M}_{\mathcal{K}^{p}}\left(E_{m}\right)<\infty$ and [Alt06, Lem. A 1.17, $\langle 2\rangle$ ], we conclude

$$
\lim _{\tau \rightarrow 0} \mathcal{I}(\tau)=0,
$$


and so we are able to pick some $0<\tau_{0} \leq \frac{1}{2 m}$ with

$$
\mathcal{I}\left(2 \tau_{0}\right) \leq \frac{\eta \mathcal{H}^{n}\left(E_{m}\right)}{2 \omega_{n} \cdot 2^{n+3}}
$$

We set

$$
\mathcal{V}:=\left\{B(x, \tau) \mid x \in E_{m}, 0<\tau<\tau_{0}, \mathcal{H}^{n}\left(E_{m} \cap B(x, \tau)\right) \geq \frac{\tau^{n} \omega_{n}}{2^{n+1}}\right\}
$$

Since

$$
0<\frac{1}{2} \mathcal{H}^{n}(E) \leq \mathcal{H}^{n}\left(E_{m}\right) \leq \mathcal{H}^{n}(E)<\infty,
$$

we get (4.1) with $E_{m}$ instead of $E$, [EG92, 2.3, Thm. 2]. This implies that for $\mathcal{H}^{n}$-almost every $x \in E_{m}$, there exists some sequence $\left(\tau_{k}\right)_{k \in \mathbb{N}}$ with $0<\tau_{k}<\tau_{0}$ and $\lim _{k \rightarrow \infty} \tau_{k}=0$ so that $\frac{\tau_{k}^{n} \omega_{n}}{2^{n+1}}<\mathcal{H}^{n}\left(E_{m} \cap B\left(x, \tau_{k}\right)\right)$ for all $k$ large enough. We obtain

$$
\inf \{\tau \mid B(x, \tau) \in \mathcal{V}\}=0 \text { for } \mathcal{H}^{n} \text {-almost every } x \in E_{m} .
$$

According to [Fal86, 1.3], $\mathcal{V}$ is a Vitali class. $B\left(x_{i}, \tau_{i}\right) \cap E_{m}$ is measurable, so for every countable, disjoint subfamily $B_{i}=B\left(x_{i}, \tau_{i}\right)$ of $\mathcal{V}$, we have

$$
\begin{aligned}
\sum_{i \in \mathbb{N}}\left(\operatorname{diam} B_{i}\right)^{n}=\sum_{i \in \mathbb{N}}\left(2 \tau_{i}\right)^{n} \leq & 2^{n} \frac{2^{n+1}}{\omega_{n}} \sum_{i \in \mathbb{N}} \mathcal{H}^{n}\left(B\left(x_{i}, \tau_{i}\right) \cap E_{m}\right) \\
& =\frac{2^{2 n+1}}{\omega_{n}} \mathcal{H}^{n}\left(\bigcup_{i \in \mathbb{N}}\left(B\left(x_{i}, \tau_{i}\right) \cap E_{m}\right)\right) \leq \frac{2^{2 n+1}}{\omega_{n}} \mathcal{H}^{n}\left(E_{m}\right)<\infty .
\end{aligned}
$$

Applying Vitali's Covering Theorem [Fal86, 1.3, Thm. 1.10], we get a countable subfamily of $\mathcal{V}$ with disjoint balls $B_{i}=B\left(x_{i}, \tau_{i}\right)$ fulfilling

$$
\mathcal{H}^{n}\left(E_{m} \backslash \bigcup_{i \in \mathbb{N}} B\left(x_{i}, \tau_{i}\right)\right)=0 .
$$

Therefore, we have

$$
\begin{aligned}
\mathcal{H}^{n}\left(E_{m}\right) & =\mathcal{H}^{n}\left[\left(E_{m} \backslash \bigcup_{i \in \mathbb{N}} B_{i}\right) \cup\left(E_{m} \cap \bigcup_{i \in \mathbb{N}} B_{i}\right)\right] \\
& \leq \mathcal{H}^{n}\left(E_{m} \backslash \bigcup_{i \in \mathbb{N}} B_{i}\right)+\mathcal{H}^{n}\left(E_{m} \cap \bigcup_{i \in \mathbb{N}} B_{i}\right) \\
& =0+\mathcal{H}^{n}\left(\bigcup_{i \in \mathbb{N}}\left(E_{m} \cap B_{i}\right)\right) \\
& \leq \sum_{i \in \mathbb{N}} \mathcal{H}^{n}\left(E_{m} \cap B_{i}\right) \\
& \leq \sum_{i \in \mathbb{N}} \mathcal{H}^{n}\left(E \cap B\left(x_{i}, \tau_{i}\right)\right) \\
& (4.2) \\
& \leq \sum_{i \in \mathbb{N}} 2 \omega_{n} \tau_{i}^{n},
\end{aligned}
$$


so that

$$
\sum_{i \in \mathbb{N}} \tau_{i}^{n} \geq \frac{\mathcal{H}^{n}\left(E_{m}\right)}{2 \omega_{n}}
$$

Furthermore, with $\left(B_{i} \cap E_{m}\right)^{n+2} \subset A\left(2 \tau_{0}\right) \cap B_{i}^{n+2}$, we obtain

$$
\begin{aligned}
\sum_{i \in \mathbb{N}} \mathcal{M}_{\mathcal{K}^{p}}\left(B_{i} \cap E_{m}\right) & =\sum_{i \in \mathbb{N}} \int_{\left(B_{i} \cap E_{m}\right)^{n+2}} \mathcal{K}^{p}\left(x_{0}, \ldots, x_{n+1}\right) \mathrm{d} \mathcal{H}^{n}\left(x_{0}\right) \ldots \mathrm{d} \mathcal{H}^{n}\left(x_{n+1}\right) \\
& \leq \sum_{i \in \mathbb{N}} \int_{A\left(2 \tau_{0}\right) \cap B_{i}^{n+2}} \mathcal{K}^{p}\left(x_{0}, \ldots, x_{n+1}\right) \mathrm{d} \mathcal{H}^{n}\left(x_{0}\right) \ldots \mathrm{d} \mathcal{H}^{n}\left(x_{n+1}\right) \\
& \leq \int_{A\left(2 \tau_{0}\right)} \mathcal{K}^{p}\left(x_{0}, \ldots, x_{n+1}\right) \mathrm{d} \mathcal{H}^{n}\left(x_{0}\right) \ldots \mathrm{d} \mathcal{H}^{n}\left(x_{n+1}\right) \\
& \stackrel{(4.4)}{=} \mathcal{I}\left(2 \tau_{0}\right) \stackrel{(4.6)}{\leq} \frac{\eta \mathcal{H}^{n}\left(E_{m}\right)}{2 \omega_{n} \cdot 2^{n+3}} .
\end{aligned}
$$

We define

$$
I_{b}:=\left\{i \in \mathbb{N} \mid \mathcal{M}_{\mathcal{K}^{p}}\left(B\left(x_{i}, \tau_{i}\right) \cap E_{m}\right) \geq \frac{\eta \tau_{i}^{n}}{2^{n+3}}\right\}
$$

and so

$$
\sum_{i \in I_{b}} \mathcal{M}_{\mathcal{K}^{p}}\left(B\left(x_{i}, \tau_{i}\right) \cap E_{m}\right) \geq \frac{\eta \sum_{i \in I_{b}} \tau_{i}^{n}}{2^{n+3}} .
$$

Assume that $\sum_{i \in I_{b}} \tau_{i}^{n}>\frac{\mathcal{H}^{n}\left(E_{m}\right)}{4 \omega_{n}}$. It follows

$$
\begin{aligned}
\sum_{i \in \mathbb{N}} \mathcal{M}_{\mathcal{K}^{p}}\left(B\left(x_{i}, \tau_{i}\right) \cap E_{m}\right) & \stackrel{(4.8)}{\leq} \frac{\eta \mathcal{H}^{n}\left(E_{m}\right)}{4 \omega_{n} \cdot 2^{n+3}} \\
& <\frac{\eta \sum_{i \in I_{b}} \tau_{i}^{2}}{2^{n+3}} \\
& \leq \sum_{i \in I_{b}} \mathcal{M}_{\mathcal{K}^{p}}\left(B\left(x_{i}, \tau_{i}\right) \cap E_{m}\right) \\
& \leq \sum_{i \in \mathbb{N}} \mathcal{M}_{\mathcal{K}^{p}}\left(B\left(x_{i}, \tau_{i}\right) \cap E_{m}\right)
\end{aligned}
$$

This is a contradiction, so

$$
\sum_{i \in I_{b}} \tau_{i}^{2} \leq \frac{\mathcal{H}^{n}\left(E_{m}\right)}{4 \omega_{n}}
$$

and with (4.7), we have $I_{b} \neq \mathbb{N}$. Now we are able to choose some $i \in \mathbb{N} \backslash I_{b}$ and get:
(a) $\mathcal{H}^{n}\left(B\left(x_{i}, \tau_{i}\right) \cap E_{m}\right) \geq \frac{\tau_{i}^{n} \omega_{n}}{2^{n+1}}$,
see definition of $\mathcal{V}$
(b) $\mathcal{M}_{\mathcal{K}^{p}}\left(B\left(x_{i}, \tau_{i}\right) \cap E_{m}\right)<\eta \frac{\tau_{i}^{n}}{2^{n+3}}$,
see definition of $I_{b}$ 
(c) For every ball $B(x, t)$ with $x \in B\left(x_{i}, \tau_{i}\right) \cap E_{m}$ and $t>0$, we obtain $\mathcal{H}^{n}\left(B\left(x_{i}, \tau_{i}\right) \cap E_{m} \cap B(x, t)\right) \leq 2 \omega_{n} t^{n}$.

Justification: Let $x \in B\left(x_{i}, \tau_{i}\right) \cap E_{m}$ and $t>0$.

1. Case: $t<\frac{1}{m}$.

Due to $x \in E_{m}$, we have $\mathcal{H}^{n}\left(B\left(x_{i}, \tau_{i}\right) \cap E_{m} \cap B(x, t)\right) \leq \mathcal{H}^{n}(E \cap B(x, t)) \stackrel{(4.2)}{\leq} 2 \omega_{n} t^{n}$.

2. Case: $t \geq \frac{1}{m}$.

Due to $x_{i} \in E_{m}$ and $\tau_{i}<\tau_{0} \leq \frac{1}{2 m}<t$, we get

$$
\mathcal{H}^{n}\left(B\left(x_{i}, \tau_{i}\right) \cap E_{m} \cap B(x, t)\right) \leq \mathcal{H}^{n}\left(B\left(x_{i}, \tau_{i}\right) \cap E\right) \stackrel{(4.2)}{\leq} 2 \omega_{n} \tau_{i}^{n} \leq 2 \omega_{n} t^{n} .
$$

The Hausdorff measure is regular [Fal86, 1.2, Thm. 1.6], so with (a) we are able to pick some closed set $E^{*} \subset B\left(x_{i}, \tau_{i}\right) \cap E_{m}$ with $\mathcal{H}^{n}\left(E^{*}\right) \geq \frac{\tau_{i}^{n} \omega_{n}}{2^{n+2}}$. Now we have

(i) $E^{*}$ is compact

because $E^{*} \subset B\left(x_{i}, \tau_{i}\right) \cap E_{m}$ is bounded and closed.

(iii) $\mathcal{H}^{n}\left(E^{*}\right)>\frac{\left(\operatorname{diam} E^{*}\right)^{n} \omega_{n}}{2^{2 n+2}}$

because $E^{*} \subset B\left(x_{i}, \tau_{i}\right)$ and so $\mathcal{H}^{n}\left(E^{*}\right)>\frac{\tau_{i}^{n} \omega_{n}}{2^{n+2}} \geq \frac{\left(\operatorname{diam} E^{*}\right)^{n}}{2^{2 n+2}}$.

(iv) $\forall x \in E^{*}, \forall t>0$, we have $\mathcal{H}^{n}\left(E^{*} \cap B(x, t)\right) \leq 2 \omega_{n} t^{n}$

because $\mathcal{H}^{n}\left(E^{*} \cap B(x, t)\right) \leq \mathcal{H}^{n}\left(B\left(x_{i}, \tau_{i}\right) \cap E_{m} \cap B(x, t)\right) \stackrel{\text { (c) }}{\leq} 2 \omega_{n} t^{n}$.

(ii) $\mathcal{M}_{\mathcal{K}^{p}}\left(E^{*}\right) \leq \eta\left(\operatorname{diam} E^{*}\right)^{n}$

because we have $E^{*} \subset B\left(\bar{x}\right.$, diam $\left.E^{*}\right)$ for some $\bar{x} \in E^{*}$ and so

$$
\tau_{i}^{n} \leq \frac{2^{n+2}}{\omega_{n}} \mathcal{H}^{n}\left(E^{*}\right)=\frac{2^{n+2}}{\omega_{n}} \mathcal{H}^{n}\left(E^{*} \cap B\left(\bar{x}, \operatorname{diam} E^{*}\right)\right) \stackrel{(\text { iv) }}{\leq} 2^{n+3}\left(\operatorname{diam} E^{*}\right)^{n} .
$$

Now we get $\mathcal{M}_{\mathcal{K}^{p}}\left(E^{*}\right) \leq \mathcal{M}_{\mathcal{K}^{p}}\left(B\left(x_{i}, \tau_{i}\right) \cap E_{m}\right) \stackrel{(\mathrm{b})}{\leq} \eta_{2_{i}^{n}}^{2^{n+3}} \leq \eta\left(\operatorname{diam} E^{*}\right)^{n}$.

Next, we present the crucial theorem of this work. For this purpose, we first have to define the notion of a proper integrand and the integral Menger curvature for Borel measures.

Definition 4.4 ( $\mu$-proper integrand). Let $\mathcal{K}:\left(\mathbb{R}^{N}\right)^{n+2} \rightarrow[0, \infty)$ and $p \geq 2$. We say $\mathcal{K}^{p}$ is a $\mu$-proper integrand if it fulfils the following four conditions:

- $\mathcal{K}$ is $(\mu)^{n+2}$-measurable, where $(\mu)^{n+2}$ means the $(n+2)$-times product measure of $\mu$.

- There exists some constants $c=c(n, \mathcal{K}, p) \geq 1$ and $l=l(n, \mathcal{K}, p) \geq 1$ so that for all $t>0, C \geq 1, x \in \mathbb{R}^{N}$ and all $\left(n, \frac{t}{C}\right)$-simplexes $\Delta\left(x_{0}, \ldots, x_{n}\right) \subset B(x, C t)$, we have

$$
\left(\frac{d\left(w, \operatorname{aff}\left(x_{0}, \ldots, x_{n}\right)\right)}{t}\right)^{p} \leq c C^{l} t^{n(n+1)} \mathcal{K}^{p}\left(x_{0}, \ldots, x_{n}, w\right),
$$

for all $w \in B(x, C t)$. 
- For all $t>0$, we have

$$
t^{n(n+1)} \mathcal{K}\left(t x_{0}, \ldots, t x_{n+1}\right)=\mathcal{K}\left(x_{0}, \ldots, x_{n+1}\right),
$$

- $\mathcal{K}$ is invariant under every translation, i.e., for every $b \in \mathbb{R}$, we have

$$
\mathcal{K}\left(x_{0}+b, \ldots, x_{n+1}+b\right)=\mathcal{K}\left(x_{0}, \ldots, x_{n+1}\right) .
$$

Definition 4.5 (Integral Menger curvature for Borel measures). Let $\mu$ be a Borel measure. We define the integral Menger curvature of $\mu$ with integrand $\mathcal{K}^{p}$ by

$$
\mathcal{M}_{\mathcal{K}^{p}}(\mu):=\int \ldots \int \mathcal{K}^{p}\left(x_{0}, \ldots, x_{n+1}\right) \mathrm{d} \mu\left(x_{0}\right) \ldots \mathrm{d} \mu\left(x_{n+1}\right) .
$$

Theorem 4.6. Let $\mathcal{K}:\left(\mathbb{R}^{N}\right)^{n+2} \rightarrow[0, \infty)$. For every $C_{0} \geq 10$, there exists some $\eta=$ $\eta\left(N, n, \mathcal{K}, C_{0}\right)>0$ so that if $\mu$ is a Borel measure on $\mathbb{R}^{N}$ with compact support $F, \mathcal{K}^{2}$ is a $\mu$-proper integrand and if they fulfil

(命) $\mu(B(0,2)) \geq 1, \mu\left(\mathbb{R}^{N} \backslash B(0,2)\right)=0$,

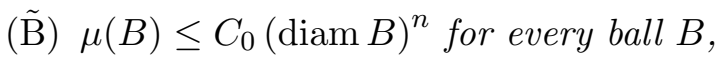

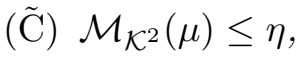

then there exists some Lipschitz graph $\Gamma$ with

$$
\mu(\Gamma) \geq \frac{99}{100} \mu\left(\mathbb{R}^{N}\right) .
$$

This statement is a direct consequence of Theorem 6.1 which is a slight modification of the current one. The main work will be the proof of Theorem 6.1 , which is done in chapter 6 to 8.

Now we can prove our main Theorem 3.4 under the assumption that the previous theorem is correct. We will use the notation $s E:=\left\{x \in \mathbb{R}^{N} \mid s^{-1} x \in E\right\}$ for $s>0$ and some set $E \subset \mathbb{R}^{N}$. Distinguish this notation from $s B(x, t)=B(x, s t)$, where the centre stays unaffected and only the radius is scaled.

Proof of Theorem 3.4. Let $\mathcal{K}^{2}$ be some proper integrand (see Definition 4.4) and $E \subset \mathbb{R}^{N}$ some Borel set with $\mathcal{M}_{\mathcal{K}^{2}}(E)<\infty$.

We start with a countable covering of $\mathbb{R}^{N}$ with balls $B_{i}$ so that $\mathbb{R}^{N} \subset \bigcup_{i \in \mathbb{N}} B_{i}$. We will show that for all $i \in \mathbb{N}$ the sets $E \cap B_{i}$ are $n$-rectifiable, which implicates that $E$ is $n$-rectifiable.

Let $i \in \mathbb{N}$ with $\mathcal{H}^{n}\left(E \cap B_{i}\right)>0$. With Lemma 4.2, we conclude that $\mathcal{H}^{n}\left(E \cap B_{i}\right)<\infty$. Then, using Lemma A.1, we can decompose $E \cap B_{i}$ into two disjoint subsets

$$
E \cap B_{i}=E_{\mathrm{r}}^{i} \dot{\cup} E_{\mathrm{u}}^{i},
$$

where $E_{\mathrm{r}}^{i}$ is $n$-rectifiable and $E_{\mathrm{u}}^{i}$ is purely $n$-unrectifiable.

Now we assume that $E \cap B_{i}$ is not $n$-rectifiable, so $\mathcal{H}^{n}\left(E_{\mathrm{u}}^{i}\right)>0$. The sets $E \cap B_{i}$ and $E_{\mathrm{r}}^{i}$ are Borel sets, so $E_{\mathrm{u}}^{i}$ is a Borel set, too and fulfils $0<\mathcal{H}^{n}\left(E_{\mathrm{u}}^{i}\right) \leq \mathcal{H}^{N}\left(E \cap B_{i}\right)<\infty$ and $\mathcal{M}_{\mathcal{K}^{2}}\left(E_{\mathrm{u}}^{i}\right) \leq \mathcal{M}_{\mathcal{K}^{2}}(E)<\infty$. Now, due to Lemma 4.3 , for every $\eta>0$, there exists some set $E^{*} \subset E_{\mathrm{u}}^{i}$ with 
(i) $E^{*}$ is compact,

(ii) $\mathcal{M}_{\mathcal{K}^{2}}\left(E^{*}\right) \leq \frac{\eta \omega_{n}^{n+2}}{2^{(2 n+2)(n+2)}}\left(\operatorname{diam} E^{*}\right)^{n}$,

(iii) $\mathcal{H}^{n}\left(E^{*}\right)>\frac{\left(\operatorname{diam} E^{*}\right)^{n} \omega_{n}}{2^{2 n+2}}$,

(iv) $\forall x \in E^{*}, \forall t>0, \mathcal{H}^{n}\left(E^{*} \cap B(x, t)\right) \leq 2 \omega_{n} t^{n}$.

We set $a:=\frac{1}{\operatorname{diam} E^{*}}>0$ and choose some $b \in \mathbb{R}^{N}$ so that $a E^{*}+b \subset B(0,2)$. Now we define the Borel measure $\mu:=\frac{2^{2 n+2}}{\omega_{n}} \mathcal{H}^{n} \mathrm{~L}\left(a E^{*}+b\right) . E^{*}$ is compact and hence, $\mu$ has a compact support. According to [EG92, 2.1, Thm. 2], we have

$$
\mathcal{H}^{n}(a A+b)=a^{n} \mathcal{H}^{n}(A) .
$$

Furthermore, we get

$(\tilde{\mathrm{A}}) \mu(B(0,2)) \geq 1, \mu\left(\mathbb{R}^{N} \backslash B(0,2)\right)=0$

because $a E^{*}+b \subset B(0,2)$ and so

$$
\mu(B(0,2))=\frac{2^{2 n+2}}{\omega_{n}} \mathcal{H}^{n}\left(a E^{*}+b\right) \stackrel{(4.9)}{=} \frac{2^{2 n+2}}{\omega_{n}} a^{n} \mathcal{H}^{n}\left(E^{*}\right) \stackrel{(\mathrm{iii})}{>} \frac{2^{2 n+2}}{\omega_{n}} a^{n} \frac{\left(\operatorname{diam} E^{*}\right)^{n} \omega_{n}}{2^{2 n+2}}=1 .
$$

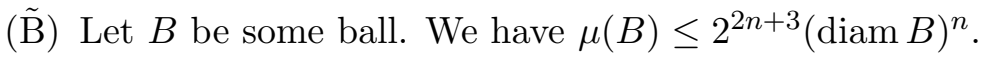

Proof:

1. Case: $B \cap\left(a E^{*}+b\right)=\emptyset$.

We have $\mu(B)=0 \leq 2^{2 n+3}(\operatorname{diam} B)^{n}$.

2. Case: $B \cap\left(a E^{*}+b\right) \neq \emptyset$.

Choose $y \in B \cap\left(a E^{*}+b\right)$. We get $B \subset B(y, \operatorname{diam} B)=: \hat{B}$ and so

$$
\begin{aligned}
\mu(B) & \leq \mu(\hat{B}) \\
& =\frac{2^{2 n+2}}{\omega_{n}} \mathcal{H}^{n}\left(\hat{B} \cap\left(a E^{*}+b\right)\right) \\
& \stackrel{(4.9)}{=} \frac{2^{2 n+2}}{\omega_{n}} a^{n} \mathcal{H}^{n}\left(\frac{1}{a}(\hat{B}-b) \cap E^{*}\right) \\
& \stackrel{\text { (iv) }}{\leq} \frac{2^{2 n+2}}{\omega_{n}} a^{n} 2 \omega_{n}\left(\frac{\operatorname{diam} \frac{1}{a} \hat{B}}{2}\right)^{n} \\
& =2^{2 n+3}(\operatorname{diam} B)^{n} .
\end{aligned}
$$

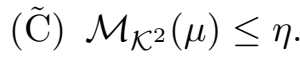

Proof. By using the third and fourth condition of a $\mu$-proper integrand and $C(n)=\left(\frac{2^{2 n+2}}{\omega_{n}}\right)^{n+2}$, we obtain applying Lemma A.3 and Lemma A.2

$$
\begin{aligned}
& \mathcal{M}_{\mathcal{K}^{2}}(\mu) \\
= & \int \ldots \int \mathcal{K}^{2}\left(x_{0}, \ldots, x_{n+1}\right) \mathrm{d} \mu\left(x_{0}\right) \ldots \mathrm{d} \mu\left(x_{n+1}\right)
\end{aligned}
$$




$$
\begin{aligned}
& =\int_{a E^{*}+b} \ldots \int_{a E^{*}+b} \mathcal{K}^{2}\left(x_{0}, \ldots, x_{n+1}\right) \mathrm{d} \frac{2^{2 n+2}}{\omega_{n}} \mathcal{H}^{n}\left(x_{0}\right) \ldots \mathrm{d} \frac{2^{2 n+2}}{\omega_{n}} \mathcal{H}^{n}\left(x_{n+1}\right) \\
& \stackrel{\mathrm{A} .3}{=} C(n) \int_{a E^{*}+b} \ldots \int_{a E^{*}+b} \mathcal{K}^{2}\left(x_{0}, \ldots, x_{n+1}\right) \mathrm{d} \mathcal{H}^{n}\left(x_{0}\right) \ldots \mathrm{d} \mathcal{H}^{n}\left(x_{n+1}\right) \\
& \stackrel{\text { A. } 2}{=} C(n) a^{n(n+2)} \int_{E^{*}} \ldots \int_{E^{*}} \mathcal{K}^{2}\left(a x_{0}+b, \ldots, a x_{n+1}+b\right) \mathrm{d} \mathcal{H}^{n}\left(x_{0}\right) \ldots \mathrm{d} \mathcal{H}^{n}\left(x_{n+1}\right) \\
& =C(n) a^{n(n+2)} \int_{E^{*}} \ldots \int_{E^{*}} \frac{1}{a^{n(n+1)}} \mathcal{K}^{2}\left(x_{0}, \ldots, x_{n+1}\right) \mathrm{d} \mathcal{H}^{n}\left(x_{0}\right) \ldots \mathrm{d} \mathcal{H}^{n}\left(x_{n+1}\right) \\
& =C(n) a^{n} \mathcal{M}_{\mathcal{K}^{2}}\left(E^{*}\right) \\
& \stackrel{\text { (ii) }}{\leq} C(n) a^{n} \frac{\eta}{C(n)}\left(\operatorname{diam} E^{*}\right)^{n} \\
& =\eta .
\end{aligned}
$$

Hence $\mu$ fulfils the requirements of Theorem 4.6 and, therefore, there exists some Lipschitz graph $\Gamma$ with

$$
\mu(\Gamma) \geq \frac{99}{100} \mu\left(\mathbb{R}^{N}\right) .
$$

The translated and scaled graph $\frac{1}{a}(\Gamma-b)$ is also a Lipschitz graph and we obtain

$$
\begin{aligned}
\mathcal{H}^{n}\left(E_{\mathrm{u}}^{i} \cap \frac{1}{a}(\Gamma-b)\right) & \geq \mathcal{H}^{n}\left(E^{*} \cap \frac{1}{a}(\Gamma-b)\right) \\
& \stackrel{(4.9)}{=} \frac{1}{a^{n}} \mathcal{H}^{n}\left(\left(a E^{*}+b\right) \cap \Gamma\right) \\
& =\frac{\omega_{n}}{2^{2 n+2} a^{n}}\left(\frac{2^{2 n+2}}{\omega_{n}} \mathcal{H}^{n} \mathrm{~L}\left(a E^{*}+b\right)\right) \\
& =\frac{\omega_{n}}{2^{2 n+2} a^{n}} \mu(\Gamma) \\
& \geq \frac{\omega_{n}}{2^{2 n+2} a^{n}} \frac{99}{100} \mu\left(\mathbb{R}^{N}\right) \\
& \stackrel{(\tilde{\mathrm{A}})}{\geq} \frac{\omega_{n}}{2^{2 n+2} a^{n}} \frac{99}{100} \\
& >0 .
\end{aligned}
$$

Hence there exists some Lipschitz continuous function $f: \mathbb{R}^{n} \rightarrow \mathbb{R}^{N}$ with image $f\left(\mathbb{R}^{n}\right)=$ $\frac{1}{a}(\Gamma-b)$ and $\mathcal{H}^{n}\left(E_{\mathrm{u}}^{i} \cap f\left(\mathbb{R}^{n}\right)\right)>0$. This is in contradiction to $E_{\mathrm{u}}^{i}$ being purely $n$-unrectifiable, so our assumption that $E \cap B_{i}$ is not $n$-rectifiable is invalid. This implies that $E \cap B_{i}$ and so $E$ is $n$-rectifiable. 


\section{$5 \beta$-numbers}

In this chapter, let $C_{0} \geq 10$ and $\mu$ a Borel measure on $\mathbb{R}^{N}$ with compact support $F$ that is upper Ahlfors regular, i.e.,

(B) for every ball $B$ we have $\mu(B) \leq C_{0}(\operatorname{diam} B)^{n}$.

If $B=B(x, r)$ is some ball in $\mathbb{R}^{N}$ with centre $x$ and radius $r$ and $t \in(0, \infty)$, then we set $t B:=B(x, t r)$. Distinguish this notation from the case $t \Upsilon=\{t z \mid z \in \Upsilon\}$ where $\Upsilon \subset \mathbb{R}^{N}$ is some arbitrary set. Furthermore, in this and the following chapters, we assume that every ball is closed. We need this to apply Vitali's and Besicovitch's covering theorems. By $C$, we denote a generic constant with a fixed value which may change from line to line.

\subsection{Measure quotient}

Definition 5.1 (Measure quotient). For a ball $B=B(x, t)$ with centre $x \in \mathbb{R}^{N}$, radius $t>0$ and a $\mu$-measurable set $\Upsilon \subset \mathbb{R}^{N}$, we define the measure quotient

$$
\delta(B \cap \Upsilon)=\delta_{\mu}(B \cap \Upsilon):=\frac{\mu(B(x, t) \cap \Upsilon)}{t^{n}}
$$

In most instances, we will use the special case

$$
\delta(B)=\delta_{\mu}(B):=\frac{\mu(B(x, t))}{t^{n}} .
$$

Moreover, we define for a fixed constant $k_{0} \geq 1$

$$
\tilde{\delta}_{k_{0}}(B)=\tilde{\delta}_{k_{0}, \mu}(B):=\sup _{y \in B\left(x, k_{0} t\right)} \delta_{\mu}(B(y, t)) .
$$

This measure quotient compares the amount of the support $F$ contained in a ball with the size of this ball. The following lemma states that if we have a lower control on the measure quotient of some ball, then we can find a not too flat simplex contained in this ball, where at each vertex we have a small ball with a lower control on its quotient measure.

Lemma 5.2. Let $0<\lambda \leq 2^{n}$ and $N_{0}=N_{0}(N)$ be the constant from Besicovitch's covering theorem A.12 depending only on the dimension $N$. There exist constants

$$
C_{1}=C_{1}\left(N, n, C_{0}, \lambda\right):=\frac{4 \cdot 120^{n} n^{n+1} N_{0} C_{0}}{\lambda}>3
$$

and

$$
C_{2}=C_{2}\left(N, n, C_{0}, \lambda\right):=\frac{2^{n+2} N_{0} C_{1}^{n}}{\lambda}>1
$$

so that for a given ball $B(x, t)$ and some $\mu$-measureable set $\Upsilon$ with $\delta(B(x, t) \cap \Upsilon) \geq \lambda$, there exists some $T=\Delta\left(x_{0}, \ldots, x_{n+1}\right) \in F \cap B(x, t) \cap \Upsilon$ so that for all $i \in\{0, \ldots, n+1\}$ 
(i) $\mathfrak{f c}_{i}(T)$ is an $\left(n, 10 n \frac{t}{C_{1}}\right)$-simplex,

(ii) $\mu\left(B\left(x_{i}, \frac{t}{C_{1}}\right) \cap B(x, t) \cap \Upsilon\right) \geq \frac{t^{n}}{C_{2}}$.

Proof. Let $B(x, t)$ be the ball with $\delta(B(x, t) \cap \Upsilon) \geq \lambda$ and

$$
\mathcal{F}:=\left\{B\left(y, \frac{t}{C_{1}}\right) \mid y \in F \cap B(x, t) \cap \Upsilon\right\} .
$$

With Besicovitch's covering theorem A.12 we get $N_{0}$ families $\mathcal{B}_{m} \subset \mathcal{F}, m=1, \ldots, N_{0}$ of disjoint balls, where $N_{0}$ depends only on the dimension $n$, so that

$$
F \cap B(x, t) \cap \Upsilon \subset \bigcup_{m=1}^{N_{0}} \bigcup_{B \in \mathcal{B}_{m}} B .
$$

For every ball $B \in \mathcal{F}$, it holds that $B \subset B(x, 2 t)$. Due to Lemma A.4, there are at most $\left(2 C_{1}\right)^{n}$ balls in $\mathcal{B}_{m}$. Now

$$
\begin{aligned}
\lambda \leq \delta(B(x, t) \cap \Upsilon) & =\frac{\mu(B(x, t) \cap \Upsilon)}{t^{n}}=\frac{\mu(F \cap B(x, t) \cap \Upsilon)}{t^{n}} \\
& \leq \frac{1}{t^{n}} \mu\left(\bigcup_{m=1}^{N_{0}} \bigcup_{B \in \mathcal{B}_{m}}(B \cap B(x, t) \cap \Upsilon)\right) \\
& \leq \frac{1}{t^{n}} \sum_{m=1}^{N_{0}} \sum_{B \in \mathcal{B}_{m}} \mu(B \cap B(x, t) \cap \Upsilon) .
\end{aligned}
$$

From this we get the existence of a family $\mathcal{B}_{m}$ with

$$
\sum_{B \in \mathcal{B}_{m}} \mu(B \cap B(x, t) \cap \Upsilon) \geq \frac{\lambda t^{n}}{N_{0}}
$$

We assume that for every $S=\Delta\left(y_{0}, \ldots, y_{n+1}\right) \in F \cap B(x, t) \cap \Upsilon$, there exists some $i \in$ $\{0, \ldots, n+1\}$ so that either $\mathfrak{f c}_{i}(S)$ is no $\left(n, 10 n \frac{t}{C_{1}}\right)$-simplex or $\mu\left(B\left(y_{i}, \frac{t}{C_{1}}\right) \cap B(x, t) \cap \Upsilon\right)<\frac{t^{n}}{C_{2}}$. We define

$$
\mathcal{G}:=\left\{B \in \mathcal{B}_{m} \mid \mu(B \cap B(x, t) \cap \Upsilon) \geq \frac{t^{n}}{C_{2}}\right\}
$$

With Lemma 2.15 (where we set $G$ as the set of centres of balls in $\mathcal{G}$ and $C=10 n \frac{t}{C_{1}}$ ), we know that there exists some $T_{z}=\Delta\left(z_{0}, \ldots, z_{n}\right)$ so that for every ball $B\left(y, \frac{t}{C_{1}}\right) \in \mathcal{G}$, there exists some $i \in\{0, \ldots, n\}$ so that $d\left(y, \operatorname{aff}\left(\mathfrak{f c}_{i}\left(T_{z}\right)\right)\right) \leq 20 n \frac{t}{C_{1}}$. We define for $i \in\{0, \ldots, n\}$

$$
\begin{aligned}
T_{i} & :=\operatorname{aff}\left(\mathfrak{f c}_{i}\left(T_{z}\right)\right) \cap B\left(\pi_{\left.\operatorname{aff}_{\left(\mathfrak{c}_{i}\right.}\left(T_{z}\right)\right)}(x), 2 t\right), \\
\mathcal{S}_{i} & :=\left\{y \in \mathbb{R}^{n} \mid d\left(y, \operatorname{aff}\left(\mathfrak{f c}_{i}\left(T_{z}\right)\right)\right) \leq \frac{30 n t}{C_{1}}, \pi_{\left.\operatorname{aff}_{\left(\mathfrak{c}_{i}\left(T_{z}\right)\right)}(y) \in T_{i}\right\}}\right.
\end{aligned}
$$

and we know

$$
B \in \mathcal{G} \Rightarrow B \subset S_{i} \text { for some } i \in\{0, \ldots, n\} .
$$

With Lemma A.5 applied to $T_{i}, s=\frac{4}{C_{1}} t<2 t=r$ and $m=n-1$, there exists a family $\mathcal{E}$ of closed balls with 
(i) ${ }^{*} \operatorname{diam} B=\frac{8}{C_{1}} t$ for all $B \in \mathcal{E}$,

(ii)* $T_{i} \subset \bigcup_{B \in \mathcal{E}} 5 B$,

(iii) $\# \mathcal{E} \leq\left(\frac{4 t}{s}\right)^{n-1}=C_{1}^{n-1}$.

Let $y \in S_{i}$. We have $d\left(y, \operatorname{aff}\left(\mathfrak{f c}_{i}\left(T_{z}\right)\right)\right) \leq \frac{30 n}{C_{1}} t$ and $\pi_{\text {aff( }\left(\mathfrak{f}_{i}\left(T_{z}\right)\right)}(y) \in T_{i}$. So, because of (ii)*, there exists some $B=B\left(z, \frac{4}{C_{1}} t\right) \in \mathcal{E}$ with $\pi_{\mathrm{aff}_{\left(\mathfrak{f}_{i}(T)\right)}}(y) \in 5 B$ and we have

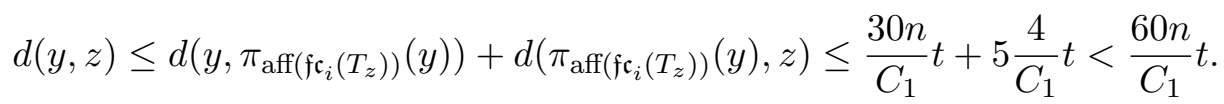

This proves $S_{i} \subset \bigcup_{B \in \mathcal{E}} 15 n B$. We therefrom derive with (B) (see page 45)

$$
\mu\left(S_{i}\right) \leq \sum_{B \in \mathcal{E}} \mu(15 n B) \stackrel{(\mathrm{B})}{\leq} \sum_{B \in \mathcal{E}} C_{0}(15 n \operatorname{diam} B)^{n} \stackrel{(\mathrm{i})^{*}}{\leq} \# \mathcal{E} C_{0} \frac{120^{n} n^{n} t^{n}}{C_{1}^{n}} \stackrel{(\mathrm{iii})^{*}}{\leq} 120^{n} n^{n} C_{0} \frac{t^{n}}{C_{1}} .
$$

We define for $i \in\{1, \ldots, n\}$

$$
\begin{aligned}
\mathcal{G}_{0} & :=\left\{B \in \mathcal{G} \mid B \subset S_{0}\right\}, \\
\mathcal{G}_{i} & :=\left\{B \in \mathcal{G} \mid B \subset S_{i} \text { and } B \notin \bigcup_{j=0}^{i-1} \mathcal{G}_{i}\right\}
\end{aligned}
$$

as a partition of $\mathcal{G}$, see (5.2). Now we have

$$
\begin{aligned}
\sum_{B \in \mathcal{G}} \mu(B \cap B(x, t) \cap \Upsilon) & =\sum_{i=0}^{n} \sum_{B \in \mathcal{G}_{i}} \mu(B \cap B(x, t) \cap \Upsilon) \\
& =\sum_{i=0}^{n} \mu\left(\bigcup_{B \in \mathcal{G}_{i}}(B \cap B(x, t) \cap \Upsilon)\right) \\
& \leq \sum_{i=0}^{n} \mu\left(S_{i}\right) \\
& (5.3) \\
& \leq n^{n+1} \cdot 120^{n} C_{0} \frac{t^{n}}{C_{1}} .
\end{aligned}
$$

Moreover, we have

$$
\sum_{B \in \mathcal{B}_{m} \backslash \mathcal{G}} \mu(B \cap B(x, t) \cap \Upsilon)<\sum_{B \in \mathcal{B}_{m} \backslash \mathcal{G}} \frac{t^{n}}{C_{2}} \stackrel{\# \mathcal{B}_{m} \leq\left(2 C_{1}\right)^{n}}{\leq}\left(2 C_{1}\right)^{n} \frac{t^{n}}{C_{2}} .
$$

All in all, we get with (5.1) and the definition of $C_{1}$ and $C_{2}$

$$
\lambda \leq N_{0} \frac{1}{t^{n}}\left(2^{n} t^{n} \frac{C_{1}^{n}}{C_{2}}+120^{n} n^{n+1} t^{n} C_{0} \frac{1}{C_{1}}\right)=N_{0}\left(2^{n} \frac{C_{1}^{n}}{C_{2}}+120^{n} n^{n+1} C_{0} \frac{1}{C_{1}}\right) \leq \frac{\lambda}{2},
$$

thus in contradiction to $\lambda>0$. This completes the proof of Lemma 5.2. 
In most instances, we will use a weaker version of Lemma 5.2:

Corollary 5.3. Let $0<\lambda \leq 2^{n}$. There exist constants $C_{1}=C_{1}\left(N, n, C_{0}, \lambda\right)>3$ and $C_{2}=C_{2}\left(N, n, C_{0}, \lambda\right)>1$ so that for a given ball $B(x, t)$ and some $\mu$-measurable set $\Upsilon$ with $\delta(B(x, t) \cap \Upsilon) \geq \lambda$, there exists some $\left(n, 10 n \frac{t}{C_{1}}\right)$-simplex $T=\Delta\left(x_{0}, \ldots, x_{n}\right) \in F \cap B(x, t) \cap \Upsilon$ so that for all $i \in\{0, \ldots, n\}$

$$
\mu\left(B\left(x_{i}, \frac{t}{C_{1}}\right) \cap B(x, t) \cap \Upsilon\right) \geq \frac{t^{n}}{C_{2}} .
$$

\section{$5.2 \beta$-numbers and integral Menger curvature}

Definition 5.4 ( $\beta$-numbers). Let $k>1$ be some fixed constant, $x \in \mathbb{R}^{N}, t>0, p \geq 1$, $\mathcal{P}(N, n)$ the set of all $n$-dimensional planes in $\mathbb{R}^{N}$ and $P \in \mathcal{P}(N, n)$. We define

$$
\begin{aligned}
& \beta_{p ; k}^{P}(x, t)=\beta_{p ; k ; \mu}^{P}(x, t):=\left(\frac{1}{t^{n}} \int_{B(x, k t)}\left(\frac{d(y, P)}{t}\right)^{p} \mathrm{~d} \mu(y)\right)^{\frac{1}{p}}, \\
& \beta_{p ; k}(x, t)=\beta_{p ; k ; \mu}(x, t):=\inf _{P \in \mathcal{P}(N, n)} \beta_{p ; k}^{P}(x, t) .
\end{aligned}
$$

The $\beta$-numbers measure how well the support of the measure $\mu$ can be approximated by some plane. A small $\beta$-number of some ball implies either a good approximation of the support by some plane or a low measure quotient $\delta$ (cf. Definition 5.1). Hence, since we are interested in good approximations by planes, we will use the $\beta$-numbers mainly for balls where we have some lower control on the measure quotient.

Definition 5.5 (Local version of $\mathcal{M}_{\mathcal{K}^{p}}$ ). For $\kappa>1, x \in \mathbb{R}^{N}, t>0, p>0$, we define

$$
\mathcal{M}_{\mathcal{K}^{p} ; \kappa}(x, t):=\int \cdots \int_{\mathcal{O}_{\kappa}(x, t)} \mathcal{K}^{p}\left(x_{0}, \ldots, x_{n+1}\right) \mathrm{d} \mu\left(x_{0}\right) \ldots \mathrm{d} \mu\left(x_{n+1}\right),
$$

where $\mathcal{K}^{p}$ is a $\mu$-proper integrand (cf. Definition 4.4 on page 41) and

$$
\mathcal{O}_{\kappa}(x, t):=\left\{\left(x_{0}, \ldots, x_{n+1}\right) \in(B(x, \kappa t))^{n+2} \mid d(a, b) \geq \frac{t}{\kappa}, \forall a, b \in\left\{x_{0}, \ldots, x_{n+1}\right\}, a \neq b\right\} .
$$

Theorem 5.6. Let $\mathcal{K}^{p}$ be a symmetric $\mu$-proper integrand and let $0<\lambda<2^{n}, k>2, k_{0} \geq 1$. There exist constants $k_{1}=k_{1}\left(N, n, C_{0}, k, k_{0}, \lambda\right)>1$ and $C=C\left(N, n, \mathcal{K}, C_{0}, k, k_{0}, \lambda\right) \geq 1$ such that if $x \in \mathbb{R}^{N}$ and $t>0$ with $\delta(B(x, t)) \geq \lambda$ for every $y \in B\left(x, k_{0} t\right)$, we have

$$
\beta_{p ; k}(y, t)^{p} \leq C \frac{\mathcal{M}_{\mathcal{K}^{p} ; k_{1}}(x, t)}{t^{n}} \leq C \frac{\mathcal{M}_{\mathcal{K}^{p} ; k_{1}+k_{0}}(y, t)}{t^{n}} .
$$

Proof. With Lemma 5.2 for $\Upsilon=\mathbb{R}^{N}$, there exists some $T=\Delta\left(x_{0}, \ldots, x_{n+1}\right) \in F \cap B(x, t)$ so that for all $i \in\{0, \ldots, n+1\}$

(i) $\mathfrak{f c}_{i}(T)$ is an $\left(n, 10 n \frac{t}{C_{1}}\right)$-simplex, 
(ii) $\mu\left(B\left(x_{i}, \frac{t}{C_{1}}\right) \cap B(x, t)\right) \geq \frac{t^{n}}{C_{2}}$,

where $C_{1}, C_{2}$ are the constants from Lemma 5.2 depending on the present constant $\lambda>0$, the constant $C_{0}$ determined in (B) on page 45 , as well as $N$ and $n$. We set $B_{i}:=B\left(x_{i}, \frac{t}{C_{1}}\right)$ and $k_{1}:=\max \left(C_{1},\left(2+k+k_{0}\right)\right)>1$.

Claim 1: Let $z_{i} \in B_{i}$ for all $i \in\{0, \ldots, n+1\}, w \in B\left(x,\left(k+k_{0}\right) t\right) \backslash \bigcup_{\substack{l=0 \\ l \neq j}}^{n+1} 2 B_{l}$ or $w \in 2 B_{j}$ for some fixed $j \in\{0, \ldots, n+1\}$. We have

$$
\left(z_{0}, \ldots, \hat{z}_{j}, \ldots, z_{n+1}, w\right) \in \mathcal{O}_{k_{1}}(x, t),
$$

where $\left(z_{0}, \ldots, \hat{z}_{j}, \ldots, z_{n+1}, w\right)$ denotes the $(n+2)$-tuple $\left(z_{0}, \ldots, z_{j-1}, z_{j+1}, \ldots, z_{n+1}, w\right)$.

Proof of Claim 1. We have for $w \in 2 B_{j}$ and $l \in\{0, \ldots, n+1\} \backslash\{j\}$ using (i) that $d\left(w, x_{l}\right) \geq$ $d\left(x_{j}, x_{l}\right)-d\left(x_{j}, w\right) \geq(10 n-2) \frac{t}{C_{1}}>2 \frac{t}{C_{1}}$. So we obtain $2 B_{j} \subset B\left(x,\left(k+k_{0}\right) t\right) \backslash \bigcup_{\substack{l=0 \\ l \neq j}}^{n+1} 2 B_{l}$. Now let $w \in B\left(x,\left(k+k_{0}\right) t\right) \backslash \bigcup_{\substack{l=0 \\ l \neq j}}^{n+1} 2 B_{l}$. Since $z_{i} \in B_{i} \subset B\left(x,\left(k+k_{0}\right) t\right)$ and $w \in B\left(x,\left(k+k_{0}\right) t\right)$, we have $\left(z_{0}, \ldots, \hat{z}_{j}, \ldots, z_{n+1}, w\right) \in B\left(x, k_{1} t\right)^{n+2}$. Furthermore, we have $\frac{t}{k_{1}} \leq 10 n \frac{t}{C_{1}} \stackrel{\text { (i) }}{\leq} d\left(z_{i}, z_{j}\right)$ and because $w \notin \bigcup_{\substack{l=0 \\ l \neq j}}^{n+1} 2 B_{l}$, we obtain $\frac{t}{k_{1}} \leq \frac{t}{C_{1}} \leq d\left(z_{i}, w\right)$ for all $i \in\{0, \ldots, n+1\} \backslash\{j\}$. Hence we get

$$
\left(z_{0}, \ldots, \hat{z}_{j}, \ldots, z_{n+1}, w\right) \in \mathcal{O}_{k_{1}}(x, t) .
$$

End of Proof of Claim 1.

Claim 2: Let $z_{i} \in B_{i}=B\left(x_{i}, \frac{t}{C_{1}}\right)$ for all $i \in\{0, \ldots, n+1\}$. Then we have

$$
\mathfrak{f c}_{i}\left(\Delta\left(z_{0}, \ldots, z_{n+1}\right)\right) \text { is an }\left(n,(9 n-1) \frac{t}{C_{1}}\right) \text {-simplex for all } i \in\{0, \ldots, n+1\} .
$$

Proof of Claim 2. Due to (i), $d\left(x_{i}, z_{i}\right) \leq \frac{t}{C_{1}}$ and Lemma 2.14 applied on $\mathfrak{f c}_{i}(T)$, the simplex $\mathfrak{f c}_{i}\left(\Delta\left(z_{0}, \ldots, z_{n+1}\right)\right)$ is an $\left(n,(9 n-1) \frac{t}{C_{1}}\right)$-simplex for all $i \in\{0, \ldots, n+1\}$.

End of Proof of Claim 2.

Claim 3: Let $z_{i} \in B_{i}=B\left(x_{i}, \frac{t}{C_{1}}\right)$ for all $i \in\{0, \ldots, n+1\}, w \in B\left(x,\left(k+k_{0}\right) t\right)$. There exists some constant $\tilde{C}=\tilde{C}\left(N, n, \mathcal{K}, p, C_{0}, k, k_{0}, \lambda\right)$ so that for all $j \in\{0, \ldots, n+1\}$, we have

$$
\left(\frac{d\left(w, \operatorname{aff}\left(z_{0}, \ldots, \hat{z}_{j}, \ldots, z_{n+1}\right)\right)}{t}\right)^{p} \leq \tilde{C} t^{n(n+1)} \mathcal{K}^{p}\left(z_{0}, \ldots, \hat{z}_{j}, \ldots, z_{n+1}, w\right) .
$$

Proof of Claim 3. $\mathcal{K}^{p}$ is a $\mu$-proper integrand. Hence with Claim 2, we get the desired estimate. End of Proof of Claim 3.

Claim 4: There exist some constant $C=C\left(N, n, \mathcal{K}, p, C_{0}, k, k_{0}, \lambda\right)$ and $z_{i} \in F \cap B_{i} \cap B(x, t)$, $i \in\{0, \ldots, n+1\}$, so that for all $l \in\{0, \ldots, n+1\}$, we have

$$
\int \mathbb{1}_{\left\{\left(z_{0}, \ldots, \hat{z}_{l}, \ldots, z_{n+1}, w\right) \in \mathcal{O}_{k_{1}}(x, t)\right\}} \mathcal{K}^{p}\left(z_{0}, \ldots, \hat{z}_{l}, \ldots, z_{n+1}, w\right) \mathrm{d} \mu(w) \leq C \frac{\mathcal{M}_{\mathcal{K}^{p} ; k_{1}}(x, t)}{t^{(n+1) n}}
$$


and with $P_{n+1}:=\operatorname{aff}\left(z_{0}, \ldots, z_{n}\right)$

$$
\left(\frac{d\left(z_{n+1}, P_{n+1}\right)}{t}\right)^{p} \leq C \frac{\mathcal{M}_{\mathcal{K}^{p} ; k_{1}}(x, t)}{t^{n}} .
$$

Proof of Claim 4. For $E \subset \mathbb{R}^{N}$ with $\# E=m+1, E=\left\{e_{0}, \ldots, e_{m}\right\}, 0 \leq m \leq n$, we set

$$
\begin{aligned}
& \mathcal{R}(E):=\int_{F^{n-m+1}} \mathbb{1}_{\left\{\left(e_{0}, \ldots, e_{m}, w_{m+1}, \ldots, w_{n+1}\right) \in \mathcal{O}_{k_{1}}(x, t)\right\}} \\
& \mathcal{K}^{p}\left(e_{0}, \ldots, e_{m}, w_{m+1}, \ldots, w_{n+1}\right) \mathrm{d} \mu\left(w_{m+1}\right) \ldots \mathrm{d} \mu\left(w_{n+1}\right) .
\end{aligned}
$$

The integrand $\mathcal{K}$ is symmetric, hence the value $\mathcal{R}(E)$ is well-defined because it does not depend on the numbering of the elements of $E$. In the following part, we use the convention that $\{0, \ldots,-1\}=\emptyset$ and $\left\{z_{0}, \ldots, z_{-1}\right\}=\emptyset$.

At first, we show by an inductive construction that, for all $m \in \mathbb{N}$ with $0 \leq m \leq n+1$, there holds:

For all $j \in\{0, \ldots, m\}$ and $i \in\{j, \ldots, n+1\}$, there exist constants $C^{(j)}>1$, sets $Z_{i}^{j} \subset$ $F \cap B_{i} \cap B(x, t)$ and, for all $l \in\{0, \ldots, m-1\}$, there exist $z_{l} \in Z_{l}^{l}$ with

$$
\mu\left(Z_{i}^{j}\right)>\frac{t^{n}}{2^{j+1} C_{2}},
$$

and, for all $u \in\{0, \ldots, m\}$, for all $E \subset\left\{z_{0}, \ldots, z_{u-1}\right\}$ and $z \in Z_{r}^{u}$, where $r \in\{u, \ldots, n+1\}$, we have

$$
\mathcal{R}(E \cup\{z\}) \leq C^{(u)} \frac{\mathcal{M}_{\mathcal{K}^{p} ; k_{1}}(x, t)}{t^{(\# E+1) n}} .
$$

We start with $m=0$ and $j=0$ and choose the constant $C^{(0)}:=2 C_{2}$, set $\Upsilon_{i}:=F \cap B_{i} \cap B(x, t)$ and define for every $i \in\{0, \ldots, n+1\}$

$$
Z_{i}^{0}:=\left\{z \in \Upsilon_{i} \mid \mathcal{R}(\{z\}) \leq C^{(0)} \frac{\mathcal{M}_{\mathcal{K}^{p} ; k_{1}}(x, t)}{t^{n}}\right\}
$$

We prove $\mu\left(Z_{i}^{0}\right)>\frac{t^{n}}{2 C_{2}}$. Due to $Z_{i}^{0} \subset \Upsilon_{i}$, we have

$$
\mu\left(\Upsilon_{i}\right)=\mu\left(Z_{i}^{0} \cup\left[\Upsilon_{i} \backslash Z_{i}^{0}\right]\right) \leq \mu\left(Z_{i}^{0}\right)+\mu\left(\Upsilon_{i} \backslash Z_{i}^{0}\right)
$$

and so

$$
\mu\left(Z_{i}^{0}\right) \geq \mu\left(\Upsilon_{i}\right)-\mu\left(\Upsilon_{i} \backslash Z_{i}^{0}\right) .
$$

Furthermore,

and we obtain

$$
\mu\left(\Upsilon_{i}\right)=\mu\left(B_{i} \cap B(x, t)\right) \stackrel{\text { (ii) }}{\geq} \frac{t^{n}}{C_{2}}
$$

$$
\begin{aligned}
\mu\left(\Upsilon_{i} \backslash Z_{i}^{0}\right) & =\left(C^{(0)} \frac{\mathcal{M}_{\mathcal{K}^{p} ; k_{1}}(x, t)}{t^{n}}\right)^{-1} \int_{\Upsilon_{i} \backslash Z_{i}^{0}} C^{(0)} \frac{\mathcal{M}_{\mathcal{K}^{p} ; k_{1}}(x, t)}{t^{n}} \mathrm{~d} \mu(z) \\
& \stackrel{(5.8)}{<}\left(C^{(0)} \frac{\mathcal{M}_{\mathcal{K}^{p} ; k_{1}}(x, t)}{t^{n}}\right)^{-1} \underbrace{\int \mathcal{R}(\{z\}) \mathrm{d} \mu(z)}_{=\mathcal{M}_{\mathcal{K}^{p} ; k_{1}}(x, t)} \\
& =\frac{t^{n}}{C^{(0)}} .
\end{aligned}
$$


Now we get for all $i \in\{0, \ldots, n+1\}$

$$
\mu\left(Z_{i}^{0}\right) \geq \mu\left(\Upsilon_{i}\right)-\mu\left(\Upsilon_{i} \backslash Z_{i}^{0}\right)>\frac{t^{n}}{C_{2}}-\frac{t^{n}}{C^{(0)}}=\frac{t^{n}}{2 C_{2}} .
$$

Let $u=0, E \subset\left\{z_{0}, \ldots, z_{-1}\right\}=\emptyset$ and $z \in Z_{r}^{0}$, where $r \in\{0, \ldots, n+1\}$. We have

$$
\mathcal{R}(E \cup\{z\}) \stackrel{(5.8)}{\leq} C^{(0)} \frac{\mathcal{M}_{\mathcal{K}^{p} ; k_{1}}(x, t)}{t^{n}} .
$$

Now let $m \in\{0, \ldots, n\}$ and we assume that for all $j \in\{0, \ldots, m\}$ and $i \in\{j, \ldots, n+1\}$, there exist constants $C^{(j)}>1$, sets $Z_{i}^{j} \subset F \cap B_{i} \cap B(x, t)$ and for all $l \in\{0, \ldots, m-1\}$ there exist $z_{l} \in Z_{l}^{l}$ with

$$
\mu\left(Z_{i}^{j}\right)>\frac{t^{n}}{2^{j+1} C_{2}}
$$

and for all $u \in\{0, \ldots, m\}$, for all $E \subset\left\{z_{0}, \ldots, z_{u-1}\right\}$ and $z \in Z_{r}^{u}$ where $r \in\{u, \ldots, n+1\}$, we have

$$
\mathcal{R}(E \cup\{z\}) \leq C^{(u)} \frac{\mathcal{M}_{\mathcal{K}^{p} ; k_{1}}(x, t)}{t^{(\# E+1) n}} .
$$

Next we start with the inductive step. From the induction hypothesis, we already have the constants $C^{(j)}$ and the sets $Z_{i}^{j}$ for $j \in\{0, \ldots, m\}$ and $i \in\{j, \ldots, n+1\}$ as well as $z_{l} \in Z_{l}^{l}$ for $l \in\{0, \ldots, m-1\}$. Since $\mu\left(Z_{m}^{m}\right)>0$, we can choose $z_{m} \in Z_{m}^{m}$. We define

$$
C^{(m+1)}:=2^{m} C^{(m)} 2^{m+2} C_{2}>C^{(m)}
$$

and, for $i \in\{m+1, \ldots, n+1\}$, we define

$$
Z_{i}^{m+1}:=\bigcap_{\substack{E \subset\left\{z_{0}, \ldots, z_{m}\right\} \\ z_{m} \in E}} \underbrace{\left\{z \in Z_{i}^{m} \mid \mathcal{R}(E \cup\{z\}) \leq C^{(m+1)} \frac{\mathcal{M}_{\mathcal{K}^{p} ; k_{1}}(x, t)}{\left.t^{(\# E+1) n}\right\}}\right.}_{=: D_{i, E}^{m}} .
$$

At first, we prove $\mu\left(Z_{i}^{m+1}\right) \geq \frac{t^{n}}{2^{m+2} C_{2}}$ for all $i \in\{m+1, \ldots, n+1\}$. Let $E \subset\left\{z_{0}, \ldots, z_{m}\right\}$ with $z_{m} \in E$. We have

$$
\begin{aligned}
\mu\left(Z_{i}^{m} \backslash D_{i, E}^{m}\right) & =\left(C^{(m+1)} \frac{\mathcal{M}_{\mathcal{K}^{p} ; k_{1}}(x, t)}{t^{(\# E+1) n}}\right)^{-1} \int_{Z_{i}^{m} \backslash D_{i, E}^{m}} C^{(m+1)} \frac{\mathcal{M}_{\mathcal{K}^{p} ; k_{1}}(x, t)}{t^{(\# E+1) n}} \mathrm{~d} \mu(z) \\
& \stackrel{(5.11)}{<}\left(C^{(m+1)} \frac{\mathcal{M}_{\mathcal{K}^{p} ; k_{1}}(x, t)}{t^{(\# E+1) n}}\right)^{-1} \underbrace{\int \mathcal{R}(E \cup\{z\}) \mathrm{d} \mu(z)}_{=\mathcal{R}\left(\left(E \backslash\left\{z_{m}\right\}\right) \cup\left\{z_{m}\right\}\right)} \\
& \stackrel{(5.10)}{\leq}\left(C^{(m+1)} \frac{\mathcal{M}_{\mathcal{K}^{p} ; k_{1}}(x, t)}{t^{(\# E+1) n}}\right)^{-1} C^{(m)} \frac{\mathcal{M}_{\mathcal{K}^{p} ; k_{1}}(x, t)}{t^{(\# E) n}} \\
& =\frac{C^{(m)}}{C^{(m+1)}} t^{n}
\end{aligned}
$$


so that

$$
\begin{aligned}
\mu\left(Z_{i}^{m} \backslash Z_{i}^{m+1}\right) & =\mu\left(Z_{i}^{m} \backslash\left(\bigcap_{\substack{E \subset\left\{z_{0}, \ldots, z_{m}\right\} \\
z m \in E}} D_{i, E}^{m}\right)\right) \\
& =\mu\left(\bigcup_{\substack{E \subset\left\{z_{0}, \ldots, z_{m}\right\} \\
z m \in E}}\left(Z_{i}^{m} \backslash D_{i, E}^{m}\right)\right) \\
& \leq \sum_{E \subset\left\{z_{0}, \ldots, z_{m}\right\}} \mu\left(Z_{i}^{m} \backslash D_{i, E}^{m}\right) \\
& \stackrel{(5.12) \sum_{E \subset\left\{z_{0}, \ldots, z_{m}\right\}}}{<} \frac{C^{(m)}}{C^{(m+1)}} t^{n} \\
& =\frac{2^{m} C^{(m)}}{C^{(m+1)}} t^{n} \\
& =\frac{1}{2^{m+2} C_{2}} t^{n} .
\end{aligned}
$$

Finally, we get

$$
\mu\left(Z_{i}^{m+1}\right) \geq \mu\left(Z_{i}^{m}\right)-\mu\left(Z_{i}^{m} \backslash Z_{i}^{m+1}\right) \stackrel{(5.9)}{>} \frac{t^{n}}{2^{m+1} C_{2}}-\frac{t^{n}}{2^{m+2} C_{2}}=\frac{t^{n}}{2^{m+2} C_{2}} .
$$

Now let $u \in\{0, \ldots, m+1\}$ and $E \subset\left\{z_{0}, \ldots, z_{u-1}\right\}$ and $z \in Z_{r}^{u}$ where $r \in\{u, \ldots, n+1\}$. We have to show that

$$
\mathcal{R}(E \cup\{z\}) \leq C^{(u)} \frac{\mathcal{M}_{\mathcal{K}^{p} ; k_{1}}(x, t)}{t^{(\# E+1) n}} .
$$

Due to the induction hypothesis and $z \in Z_{r}^{m+1} \subset Z_{r}^{v}$ for all $v \in\{0, \ldots, m+1\}$, we only have to consider the case $u=m+1$ and $z_{m} \in E$. Then the inequality follows from (5.11).

End of induction.

Now we construct $z_{n+1}$.

We set $P_{n+1}:=\operatorname{aff}\left(z_{0}, \ldots, z_{n}\right), \hat{C}^{(n+1)}:=\tilde{C} C^{(n)} 2^{n+3} C_{2}$, where $\tilde{C}$ is the constant from Claim 3, and define

$$
\hat{Z}_{n+1}^{n+1}:=\left\{z \in Z_{n+1}^{n+1} \mid\left(\frac{d\left(z, P_{n+1}\right)}{t}\right)^{p} \leq \hat{C}^{(n+1)} \frac{\mathcal{M}_{\mathcal{K}^{p} ; k_{1}}(x, t)}{t^{n}}\right\}
$$

Next we show $\mu\left(\hat{Z}_{n+1}^{n+1}\right) \geq \frac{t^{n}}{2^{n+3} C_{2}}>0$. Let $u \in Z_{n+1}^{n+1} \backslash \hat{Z}_{n+1}^{n+1} \subset B_{n+1} \subset B\left(x,\left(k+k_{0}\right) t\right)$. With Claim 3 applied on $w=u$ and $j=n+1$, we get

$$
\left(\frac{d\left(u, P_{n+1}\right)}{t}\right)^{p} \leq \tilde{C} t^{n(n+1)} \mathcal{K}^{p}\left(z_{0}, \ldots, z_{n}, u\right) .
$$


Now we have

$$
\begin{aligned}
& \mu\left(Z_{n+1}^{n+1} \backslash \hat{Z}_{n+1}^{n+1}\right) \\
& =\left(\hat{C}^{(n+1)} \frac{\mathcal{M}_{\mathcal{K}^{p} ; k_{1}}(x, t)}{t^{n}}\right)^{-1} \int_{Z_{n+1}^{n+1} \backslash \hat{Z}_{n+1}^{n+1}} \hat{C}^{(n+1)} \frac{\mathcal{M}_{\mathcal{K}^{p} ; k_{1}}(x, t)}{t^{n}} \mathrm{~d} \mu(u) \\
& \stackrel{(5.13)}{<}\left(\hat{C}^{(n+1)} \frac{\mathcal{M}_{\mathcal{K}^{p} ; k_{1}}(x, t)}{t^{n}}\right)^{-1} \int_{Z_{n+1}^{n+1} \backslash \hat{Z}_{n+1}^{n+1}}\left(\frac{d\left(u, P_{n+1}\right)}{t}\right)^{p} \mathrm{~d} \mu(u) \\
& \stackrel{(5.14)}{\leq}\left(\hat{C}^{(n+1)} \frac{\mathcal{M}_{\mathcal{K}^{p} ; k_{1}}(x, t)}{t^{n}}\right)^{-1} \tilde{C} t^{n(n+1)} \int_{Z_{n+1}^{n+1} \backslash \hat{Z}_{n+1}^{n+1}} \mathcal{K}^{p}\left(z_{0}, \ldots, z_{n}, u\right) \mathrm{d} \mu(u) \\
& \stackrel{\text { Claim } 1}{\leq}\left(\hat{C}^{(n+1)} \frac{\mathcal{M}_{\mathcal{K}^{p} ; k_{1}}(x, t)}{t^{n}}\right)^{-1} \tilde{C} t^{n(n+1)} \underbrace{\int \mathbb{1}_{\left\{\left(z_{0}, \ldots, z_{n}, u\right) \in \mathcal{O}_{k_{1}}(x, t)\right\}} \mathcal{K}^{p}\left(z_{0}, \ldots, z_{n}, u\right) \mathrm{d} \mu(u)}_{=\mathcal{R}\left(\left\{z_{0}, \ldots, z_{n-1}\right\} \cup\left\{z_{n}\right\}\right)} \\
& \stackrel{(5.7)}{\leq}\left(\hat{C}^{(n+1)} \frac{\mathcal{M}_{\mathcal{K}^{p} ; k_{1}}(x, t)}{t^{n}}\right)^{-1} \tilde{C} t^{n(n+1)} C^{(n)} \frac{\mathcal{M}_{\mathcal{K}^{p} ; k_{1}}(x, t)}{t^{n(n+1)}} \\
& =\frac{t^{n}}{2^{n+3} C_{2}}
\end{aligned}
$$

so that

$$
\mu\left(\hat{Z}_{n+1}^{n+1}\right) \geq \mu\left(Z_{n+1}^{n+1}\right)-\mu\left(Z_{n+1}^{n+1} \backslash \hat{Z}_{n+1}^{n+1}\right) \stackrel{(5.6)}{\geq} \frac{t^{n}}{2^{n+2} C_{2}}-\frac{t^{n}}{2^{n+3} C_{2}}=\frac{t^{n}}{2^{n+3} C_{2}}>0,
$$

and we are able to choose $z_{n+1} \in \hat{Z}_{n+1}^{n+1} \subset Z_{n+1}^{n+1}$ with

$$
\left(\frac{d\left(z_{n+1}, P_{n+1}\right)}{t}\right)^{p} \stackrel{(5.13)}{\leq} \hat{C}^{(n+1)} \frac{\mathcal{M}_{\mathcal{K}^{p} ; k_{1}}(x, t)}{t^{n}}
$$

Let $l \in\{0, \ldots, n+1\}$ and $E=\left\{z_{0}, \ldots, z_{n+1}\right\} \backslash\left\{z_{l}\right\}$. Next we show $\mathcal{R}(E) \leq C^{(n+1)} \frac{\mathcal{M}_{\mathcal{K}^{p} ; k_{1}}(x, t)}{t^{(n+1) n}}$.

1. Case: $l=n+1$.

We have

$$
\mathcal{R}(E)=\mathcal{R}\left(\left\{z_{0}, \ldots, z_{n-1}\right\} \cup\left\{z_{n}\right\}\right) \stackrel{(5.7)}{\leq} C^{(n)} \frac{\mathcal{M}_{\mathcal{K}^{p} ; k_{1}}(x, t)}{t^{(n+1) n}} \leq C^{(n+1)} \frac{\mathcal{M}_{\mathcal{K}^{p} ; k_{1}}(x, t)}{t^{(n+1) n}} .
$$

2. Case: $l \neq n+1$.

We set $E^{\prime}:=E \backslash\left\{z_{n+1}\right\} \subset\left\{z_{0}, \ldots, z_{n}\right\}$ and deduce using $z_{n+1} \in Z_{n+1}^{n+1}$

$$
\mathcal{R}(E)=\mathcal{R}\left(E^{\prime} \cup\left\{z_{n+1}\right\}\right) \stackrel{(5.7)}{\leq} C^{(n+1)} \frac{\mathcal{M}_{\mathcal{K}^{p} ; k_{1}}(x, t)}{t^{\left(\# E^{\prime}+1\right) n}}=C^{(n+1)} \frac{\mathcal{M}_{\mathcal{K}^{p} ; k_{1}}(x, t)}{t^{(n+1) n}} .
$$

All in all, we get for all $l \in\{0, \ldots, n+1\}$ and some constant $C=C\left(N, n, \mathcal{K}, p, C_{0}, k, k_{0}, \lambda\right)$

$$
\int \mathbb{1}_{\left\{\left(z_{0}, \ldots, \hat{z}_{l}, \ldots, z_{n+1}, w\right) \in \mathcal{O}_{k_{1}}(x, t)\right\}} \mathcal{K}^{p}\left(z_{0}, \ldots, \hat{z}_{l}, \ldots, z_{n+1}, w\right) \mathrm{d} \mu(w)=\mathcal{R}(E) \leq C \frac{\mathcal{M}_{\mathcal{K}^{p} ; k_{1}}(x, t)}{t^{(n+1) n}} .
$$


End of Proof of Claim 4.

With Claim 4, there exist some $z_{i} \in F \cap B_{i} \cap B(x, t), i \in\{0, \ldots, n+1\}$ fulfilling (5.4) and (5.5). Let $w \in\left(F \cap B\left(x,\left(k+k_{0}\right) t\right)\right) \backslash \bigcup_{j=0}^{n} 2 B_{j}$. With Claim 3 and $P_{n+1}=\operatorname{aff}\left(z_{0}, \ldots, z_{n}\right)$, we obtain

$$
\left(\frac{d\left(w, P_{n+1}\right)}{t}\right)^{p} \leq \tilde{C} t^{n(n+1)} \mathcal{K}^{p}\left(z_{0}, \ldots, z_{n}, w\right)
$$

Hence we get

$$
\begin{aligned}
& \int_{B\left(x,\left(k+k_{0}\right) t\right) \backslash \bigcup_{j=0}^{n} 2 B_{j}}\left(\frac{d\left(w, P_{n+1}\right)}{t}\right)^{p} \mathrm{~d} \mu(w) \\
\stackrel{(5.15)}{<} & \tilde{C} t^{n(n+1)} \int_{B\left(x,\left(k+k_{0}\right) t\right) \backslash \bigcup_{j=0}^{n} 2 B_{j}} \mathcal{K}^{p}\left(z_{0}, \ldots, z_{n}, w\right) \mathrm{d} \mu(w) \\
\stackrel{\text { Claim } 1}{\leq} & \tilde{C} t^{n(n+1)} \int \mathbb{1}_{\left\{\left(z_{0}, \ldots, z_{n}, w\right) \in \mathcal{O}_{k_{1}}(x, t)\right\}} \mathcal{K}^{p}\left(z_{0}, \ldots, z_{n}, w\right) \mathrm{d} \mu(w) \\
\stackrel{(5.4)}{\leq} & \tilde{C} t^{n(n+1)} C\left(N, n, \mathcal{K}, p, C_{0}, k, k_{0}, \lambda\right) \frac{\mathcal{M}_{\mathcal{K}^{p} ; k_{1}}(x, t)}{t^{n(n+1)}} \\
= & C\left(N, n, \mathcal{K}, p, C_{0}, k, k_{0}, \lambda\right) \mathcal{M}_{\mathcal{K}^{p} ; k_{1}}(x, t) .
\end{aligned}
$$

Now we prove this estimate on the ball $2 B_{j}$, where $j \in\{0, \ldots, n\}$. We define the plain $P_{j}:=\operatorname{aff}\left(\left\{z_{0}, \ldots, z_{n+1}\right\} \backslash\left\{z_{j}\right\}\right)$, choose $w \in 2 B_{j} \subset B\left(x,\left(k+k_{0}\right) t\right)$ and get with Claim 3

$$
\left(\frac{d\left(w, P_{j}\right)}{t}\right)^{p} \leq \tilde{C} t^{n(n+1)} \mathcal{K}^{p}\left(z_{0}, \ldots, \hat{z}_{j}, \ldots, z_{n+1}, w\right)
$$

and analogous to (5.16), we obtain

$$
\begin{aligned}
& \int_{2 B_{j}}\left(\frac{d\left(w, P_{j}\right)}{t}\right)^{p} \mathrm{~d} \mu(w) \\
< & \tilde{C} t^{n(n+1)} \int_{2 B_{j}} \mathcal{K}^{p}\left(z_{0}, \ldots, \hat{z}_{j}, \ldots, z_{n+1}, w\right) \mathrm{d} \mu(w) \\
\stackrel{\text { Claim } 1}{\leq} & \tilde{C} t^{n(n+1)} \int \mathbb{1}_{\left\{\left(z_{0}, \ldots, \hat{z}_{j}, \ldots, z_{n+1}, w\right) \in \mathcal{O}_{k_{1}}(x, t)\right\}} \mathcal{K}^{p}\left(z_{0}, \ldots, \hat{z}_{j}, \ldots, z_{n+1}, w\right) \mathrm{d} \mu(w) \\
\stackrel{(5.4)}{\leq} & \tilde{C} t^{n(n+1)} C\left(N, n, \mathcal{K}, p, C_{0}, k, k_{0}, \lambda\right) \frac{\mathcal{M}_{\mathcal{K}^{p} ; k_{1}}(x, t)}{t^{n(n+1)}} \\
= & C\left(N, n, \mathcal{K}, p, C_{0}, k, k_{0}, \lambda\right) \mathcal{M}_{\mathcal{K}^{p} ; k_{1}}(x, t) .
\end{aligned}
$$

Now we have an estimate on the ball $2 B_{j}$ but with plane $P_{j}$ instead of $P_{n+1}$. It might be that $z_{n+1} \in P_{n+1}$. In this case, we have $P_{n+1}=P_{j}$ for all $j \in\{0, \ldots, n+1\}$ and we can conclude

$$
\int_{2 B_{j}}\left(\frac{d\left(w, P_{n+1}\right)}{t}\right)^{p} \mathrm{~d} \mu(w)=\int_{2 B_{j}}\left(\frac{d\left(w, P_{j}\right)}{t}\right)^{p} \mathrm{~d} \mu(w) \stackrel{(5.17)}{\leq} C \mathcal{M}_{\mathcal{K}^{p} ; k_{1}}(x, t) .
$$


From now on, we assume that $z_{n+1} \notin P_{n+1}$. We set $w^{\prime}:=\pi_{P_{j}}(w), w^{\prime \prime}:=\pi_{P_{n+1}}\left(w^{\prime}\right)$ and deduce

$$
\begin{aligned}
d\left(w, P_{n+1}\right)^{p} & \leq d\left(w, w^{\prime \prime}\right)^{p} \\
& \leq 2^{p-1}\left(d\left(w, w^{\prime}\right)^{p}+d\left(w^{\prime}, w^{\prime \prime}\right)^{p}\right) \\
& =2^{p-1}\left(d\left(w, P_{j}\right)^{p}+d\left(w^{\prime}, P_{n+1}\right)^{p}\right) .
\end{aligned}
$$

If $d\left(w^{\prime}, P_{n+1}\right)>0$, i.e., $w^{\prime} \notin P_{n+1}$, we gain with Corollary $2.5\left(P_{1}=P_{j}, P_{2}=P_{n+1}, a_{1}=w^{\prime}\right.$, $\left.a_{2}=z_{n+1}\right)$ where $P_{j, n+1}:=P_{j} \cap P_{n+1}$

$$
\begin{aligned}
\frac{d\left(w^{\prime}, P_{n+1}\right)}{d\left(w^{\prime}, P_{j, n+1}\right)} & =\frac{d\left(z_{n+1}, P_{n+1}\right)}{d\left(z_{n+1}, P_{j, n+1}\right)} \\
\Leftrightarrow \quad d\left(w^{\prime}, P_{n+1}\right) & =d\left(z_{n+1}, P_{n+1}\right) \frac{d\left(w^{\prime}, P_{j, n+1}\right)}{d\left(z_{n+1}, P_{j, n+1}\right)} .
\end{aligned}
$$

With $l \in\{0, \ldots, n\}, l \neq j$ and Lemma $2.6\left(\pi_{P_{j, n+1}}\left(\pi_{P_{j}}(w)\right)=\pi_{P_{j, n+1}}(w)\right)$, we get

$$
\begin{aligned}
d\left(w^{\prime}, P_{j, n+1}\right) & =d\left(w^{\prime}, \pi_{P_{j, n+1}}\left(w^{\prime}\right)\right) \\
& =d\left(\pi_{P_{j}}(w), \pi_{P_{j}}\left(\pi_{P_{j, n+1}}\left(\pi_{P_{j}}(w)\right)\right)\right) \\
& \leq d\left(w, \pi_{P_{j, n+1}}\left(\pi_{P_{j}}(w)\right)\right) \\
& \stackrel{L .2 .6}{=} d\left(w, P_{j, n+1}\right) \\
& \leq d\left(w, z_{l}\right) \\
& \leq d(w, x)+d\left(x, x_{l}\right)+d\left(x_{l}, z_{l}\right) \\
& \leq\left(k+k_{0}\right) t+t+\frac{t}{C_{1}} \leq k_{1} t
\end{aligned}
$$

( $k_{1}$ is defined on page 49$)$. Claim 2 yields that $\mathfrak{f c}_{j}\left(\Delta\left(z_{0}, \ldots, z_{n+1}\right)\right)$ is an $\left(n,(9 n-1) \frac{t}{C_{1}}\right)$ simplex and we obtain

$$
\begin{aligned}
\left(\frac{d\left(w^{\prime}, P_{n+1}\right)}{t}\right)^{p} & \stackrel{(5.19)}{=}\left(\frac{d\left(z_{n+1}, P_{n+1}\right)}{t} \frac{d\left(w^{\prime}, P_{j, n+1}\right)}{d\left(z_{n+1}, P_{j, n+1}\right)}\right)^{p} \\
& \leq\left(\frac{d\left(z_{n+1}, P_{n+1}\right)}{t} \frac{k_{1} t C_{1}}{(9 n-1) t}\right)^{p} \\
& \stackrel{(5.5)}{\leq} C\left(N, n, \mathcal{K}, p, C_{0}, k, k_{0}, \lambda\right) \frac{\mathcal{M}_{\mathcal{K}^{p} ; k_{1}}(x, t)}{t^{n}}
\end{aligned}
$$

If $d\left(w^{\prime}, P_{n+1}\right)=0$, this inequality is trivially true.

Finally, we obtain with $\mu\left(2 B_{j}\right) \stackrel{(\mathrm{B})}{\leq} C_{0}\left(\operatorname{diam}\left(2 B_{j}\right)\right)^{n} \leq C_{0}\left(\frac{4 t}{C_{1}}\right)^{n}((\mathrm{~B})$ from page 45$)$

$$
\begin{aligned}
& \int_{2 B_{j}}\left(\frac{d\left(w, P_{n+1}\right)}{t}\right)^{p} \mathrm{~d} \mu(w) \\
\stackrel{(5.18)}{\leq} & 2^{p-1} \int_{2 B_{j}}\left(\frac{d\left(w, P_{j}\right)}{t}\right)^{p} \mathrm{~d} \mu(w)+2^{p-1} \int_{2 B_{j}}\left(\frac{d\left(w^{\prime}, P_{n+1}\right)}{t}\right)^{p} \mathrm{~d} \mu(w)
\end{aligned}
$$




$$
\begin{aligned}
\stackrel{(5.17)(5.20)}{\leq} & 2^{p-1} C\left(N, n, \mathcal{K}, p, C_{0}, k, k_{0}, \lambda\right) \mathcal{M}_{\mathcal{K}^{p} ; k_{1}}(x, t) \\
& +2^{p-1} C\left(N, n, \mathcal{K}, p, C_{0}, k, k_{0}, \lambda\right) \frac{\mathcal{M}_{\mathcal{K}^{p} ; k_{1}}(x, t)}{t^{n}} \mu\left(2 B_{j}\right) \\
\leq \quad & C\left(N, n, \mathcal{K}, p, C_{0}, k, k_{0}, \lambda\right) \mathcal{M}_{\mathcal{K}^{p} ; k_{1}}(x, t) .
\end{aligned}
$$

It follows that

$$
\begin{aligned}
\int_{B\left(x,\left(k+k_{0}\right) t\right)}\left(\frac{d\left(w, P_{n+1}\right)}{t}\right)^{p} \mathrm{~d} \mu(w)= & \int_{B\left(x,\left(k+k_{0}\right) t\right) \backslash\left(\bigcup_{j=0}^{n} 2 B_{j}\right)}\left(\frac{d\left(w, P_{n+1}\right)}{t}\right)^{p} \mathrm{~d} \mu(w) \\
& +\sum_{j=0}^{n} \int_{2 B_{j}}\left(\frac{d\left(w, P_{n+1}\right)}{t}\right)^{p} \mathrm{~d} \mu(w) \\
\leq & C\left(N, n, \mathcal{K}, p, C_{0}, k, k_{0}, \lambda\right) \mathcal{M}_{\mathcal{K}^{p} ; k_{1}}(x, t) .
\end{aligned}
$$

Given that $B(y, k t) \subset B\left(x,\left(k+k_{0}\right) t\right)$, we get

$$
\begin{aligned}
\beta_{p ; k}(y, t)^{p} & =\inf _{P \in \mathcal{P}(N, n)} \frac{1}{t^{n}} \int_{B(y, k t)}\left(\frac{d(w, P)}{t}\right)^{p} \mathrm{~d} \mu(w) \\
& \leq \frac{1}{t^{n}} \int_{B(y, k t)}\left(\frac{d\left(w, P_{n+1}\right)}{t}\right)^{p} \mathrm{~d} \mu(w) \\
& \leq \frac{1}{t^{n}} \int_{B\left(x,\left(k+k_{0}\right) t\right)}\left(\frac{d\left(w, P_{n+1}\right)}{t}\right)^{p} \mathrm{~d} \mu(w) \\
& \leq C\left(N, n, \mathcal{K}, p, C_{0}, k, k_{0}, \lambda\right) \frac{\mathcal{M}_{\mathcal{K}^{p} ; k_{1}}(x, t)}{t^{n}} .
\end{aligned}
$$

To obtain the main result of this theorem, the only thing left to show is $\mathcal{O}_{k_{1}}(x, t) \subset \mathcal{O}_{k_{1}+k_{0}}(y, t)$ : Let $\left(z_{0}, \ldots, z_{n+1}\right) \in \mathcal{O}_{k_{1}}(x, t)$. It follows that $z_{0}, \ldots, z_{n+1} \in B\left(x, k_{1} t\right) \subset B\left(y,\left(k_{0}+k_{1}\right) t\right)$ and $d\left(z_{i}, z_{j}\right) \geq \frac{t}{k_{1}} \geq \frac{t}{k_{1}+k_{0}}$ with $i \neq j$ and $i, j=0, \ldots, n$. Thus $\left(z_{0}, \ldots, z_{n+1}\right) \in \mathcal{O}_{k_{1}+k_{0}}(y, t)$.

Finally, we get with $C=C\left(N, n, \mathcal{K}, p, C_{0}, k, k_{0}, \lambda\right)$

$$
\beta_{p ; k}(y, t)^{p} \leq C \frac{\mathcal{M}_{\mathcal{K}^{p} ; k_{1}}(x, t)}{t^{n}} \leq C \frac{\mathcal{M}_{\mathcal{K}^{p} ; k_{1}+k_{0}}(y, t)}{t^{n}} .
$$

Theorem 5.7. Let $0<\lambda<2^{n}, k>2, k_{0} \geq 1$ and $\mathcal{K}^{p}$ be some $\mu$-proper symmetric integrand (see Definition 4.4). There exists a constant $C=C\left(N, n, \mathcal{K}, p, C_{0}, k, k_{0}, \lambda\right)$ such that

$$
\iint_{0}^{\infty} \beta_{p ; k}(x, t)^{p} \mathbb{1}_{\left\{\tilde{\delta}_{k_{0}}(B(x, t)) \geq \lambda\right\}} \frac{\mathrm{d} t}{t} \mathrm{~d} \mu(x) \leq C \mathcal{M}_{\mathcal{K}^{p}}(\mu) .
$$

Proof. At first, we prove some intermediate results.

I. Let $x \in F, t>0$ and $\tilde{\delta}_{k_{0}}(B(x, t)) \geq \lambda$. There exist some constants $k_{1}=k_{1}\left(N, n, C_{0}, k, k_{0}, \lambda\right)$ and $C=C\left(N, n, \mathcal{K}, C_{0}, k, k_{0}, \lambda\right)$ so that with $k_{2}:=k_{1}+k_{0}$, we obtain

$$
\beta_{p ; k}(x, t)^{p} \leq C \frac{\mathcal{M}_{\mathcal{K}^{p} ; k_{1}+k_{0}}(x, t)}{t^{n}}=C \frac{\mathcal{M}_{\mathcal{K}^{p} ; k_{2}}(x, t)}{t^{n}} .
$$


Proof. We have

$$
\tilde{\delta}_{k_{0}}(B(x, t))=\sup _{z \in B\left(x, k_{0} t\right)} \frac{\mu(B(z, t))}{t^{n}} \geq \lambda
$$

and hence there exists some $z \in B\left(x, k_{0} t\right)$ with $\delta(B(z, t))=\frac{\mu(B(z, t))}{t^{n}} \geq \frac{\lambda}{2}$. With Theorem 5.6, there exists some constants $k_{1}>1$ and $C \geq 1$ so that for all $y \in B\left(z, k_{0} t\right)$

$$
\beta_{p ; k}(y, t)^{p} \leq C \frac{\mathcal{M}_{\mathcal{K}^{p} ; k_{1}+k_{0}}(y, t)}{t^{n}}
$$

In particular, we have

$$
\beta_{p ; k}(x, t)^{p} \leq C \frac{\mathcal{M}_{\mathcal{K}^{p} ; k_{1}+k_{0}}(x, t)}{t^{n}}
$$

because $x \in B\left(z, k_{0} t\right)$.

II. Let $u_{0}, \ldots, u_{n+1} \in F$ and

$$
(x, t) \in \mathcal{D}_{\kappa}\left(u_{0}, \ldots, u_{n+1}\right):=\left\{(y, s) \in F \times(0, \infty) \mid\left(u_{0}, \ldots, u_{n+1}\right) \in \mathcal{O}_{\kappa}(y, s)\right\} .
$$

We have $\left(u_{0}, \ldots, u_{n+1}\right) \in \mathcal{O}_{\kappa}(x, t)$ and so $\frac{d\left(u_{0}, u_{1}\right)}{2 \kappa} \leq t \leq \kappa d\left(u_{0}, u_{1}\right)$ as well as $x \in$ $B\left(u_{0}, \kappa t\right)$.

III. Let $u_{0}, \ldots, u_{n+1} \in F$. Then the set $\mathcal{D}_{\kappa}\left(u_{0}, \ldots, u_{n+1}\right)$ is closed in $F \times \mathbb{R}$ since with $B(y, s)$ we always denote a closed ball.

IV. Let $x \in F$ and set

$$
\Upsilon_{x}:=\left\{\left(u_{0}, \ldots, u_{n+1}, t\right) \in F^{n+2} \times(0, \infty) \mid\left(u_{0}, \ldots, u_{n+1}\right) \in \mathcal{O}_{\kappa}(x, t)\right\} .
$$

Then the set $\Upsilon_{x} \cup\{(x, \ldots, x, 0)\}$ is closed in $\left(\mathbb{R}^{N}\right)^{n+2} \times \mathbb{R}$ and so the set $\Upsilon_{x}$ is closed in $\left(\mathbb{R}^{N}\right)^{n+2} \times(0, \infty)$. This implies that $\Upsilon_{x}$ is $\left(X_{i=0}^{n+1} \mu\right) \times \frac{\mathrm{d} t}{t}$-measurable because, as a product of Borel measures, this is a Borel measure as well (cf. Lemma A.11).

V. Let $u_{0}, \ldots, u_{n+1} \in F$. The function $t \mapsto \frac{1}{t^{n}}$ is $\frac{\mathrm{d} t}{t}$-measurable on $(0, \infty)$ and so, due to Lemma A.10, the function $(x, t) \mapsto \frac{1}{t^{n}}$ is $\left(\mu \times \frac{\mathrm{d} t}{t}\right)$-measurable on $\mathbb{R}^{N} \times(0, \infty)$. Moreover, we have that $\mathcal{D}_{\kappa}\left(u_{0}, \ldots, u_{n+1}\right)$ is closed (see III), which implies that $(x, t) \mapsto$ $\chi_{\mathcal{D}_{\kappa}\left(u_{0}, \ldots, u_{n+1}\right)}(x, t)$ is $\left(\mu \times \frac{\mathrm{d} t}{t}\right)$-measurable on $\mathbb{R}^{N} \times(0, \infty)$. Hence we conclude that $(x, t) \mapsto \chi_{\mathcal{D}_{\kappa}\left(u_{0}, \ldots, u_{n+1}\right)}(x, t) \frac{1}{t^{n}}$ is $\left(\mu \times \frac{\mathrm{d} t}{t}\right)$-measurable, which we need in the following to 
apply Fubini's theorem [EG92, 1.4, Thm. 1]. With condition (B) from page 45 we get

$$
\begin{aligned}
& \int_{F} \int_{0}^{\infty} \chi_{\mathcal{D}_{k_{2}}\left(u_{0}, \ldots, u_{n+1}\right)}(x, t) \frac{1}{t^{n}} \frac{\mathrm{d} t}{t} \mathrm{~d} \mu(x) \\
= & \int_{0}^{\infty} \int_{F} \chi_{\mathcal{D}_{k_{2}}\left(u_{0}, \ldots, u_{n+1}\right)}(x, t) \frac{1}{t^{n}} \mathrm{~d} \mu(x) \frac{\mathrm{d} t}{t} \\
\leq & \int_{\frac{d\left(u_{0}, u_{1}\right)}{2 k_{2}}}^{k_{2}} \frac{1}{t^{n}} \underbrace{\int_{B\left(u_{0}, k_{2} t\right)} 1 \mathrm{~d} \mu(x)}_{=\mu\left(B\left(u_{0}, k_{2} t\right)\right)} \frac{\mathrm{d} t}{t} \\
\stackrel{\text { (B) }}{\leq} & \left(2 k_{2}\right)^{n} C_{0} \int_{\frac{d\left(u_{0}, u_{1}\right)}{2 k_{2}}}^{k_{2} d\left(u_{0}, u_{1}\right)} \frac{\mathrm{d} t}{t} \\
= & \left(2 k_{2}\right)^{n}\left(\ln \left(2 k_{2}^{2}\right)\right)^{\frac{p}{2}} C_{0} .
\end{aligned}
$$

With a similar argumentation as in V using IV and $\chi_{\mathcal{D}_{k_{2}}\left(u_{0}, \ldots, u_{n+1}\right)}(x, t)=\chi_{\Upsilon_{x}}\left(u_{0}, \ldots, u_{n+1}, t\right)$, we obtain for some fixed $x \in F$ that the map

$$
\left(u_{0}, \ldots, u_{n+1}, t\right) \mapsto \chi_{\mathcal{D}_{k_{2}}\left(u_{0}, \ldots, u_{n+1}\right)}(x, t) \frac{\mathcal{K}^{p}\left(u_{0}, \ldots, u_{n+1}\right)}{t^{n}}
$$

is $\left(\left(\mathrm{X}_{i=0}^{n+1} \mu\right) \times \frac{\mathrm{d} t}{t}\right)$-measurable. Now we deduce with $k_{2}=k_{1}+k_{0}$ and Fubini's theorem [EG92, 1.4, Thm. 1]

$$
\begin{aligned}
& \int_{F} \int_{0}^{\infty} \beta_{p ; k}(x, t)^{p} \mathbb{1}_{\left\{\tilde{\delta}_{k_{0}}(B(x, t)) \geq \lambda\right\}} \frac{\mathrm{d} t}{t} \mathrm{~d} \mu(x) \\
\leq & C \int_{F} \int_{0}^{\infty} \frac{\mathcal{M}_{\mathcal{K}^{p} ; k_{2}}(x, t)}{t^{n}} \frac{\mathrm{d} t}{t} \mathrm{~d} \mu(x) \\
= & C \int_{F} \int_{0}^{\infty} \int \cdots \int_{\mathcal{O}_{k_{2}}(x, t)} \frac{\mathcal{K}^{p}\left(u_{0}, \ldots, u_{n+1}\right)}{t^{n}} \mathrm{~d} \mu\left(u_{0}\right) \ldots \mathrm{d} \mu\left(u_{n+1}\right) \frac{\mathrm{d} t}{t} \mathrm{~d} \mu(x) \\
= & C \int_{F} \int_{0}^{\infty} \int \cdots \int_{F^{n+2}} \chi_{\mathcal{D}_{k_{2}}\left(u_{0}, \ldots, u_{n+1}\right)}(x, t) \frac{\mathcal{K}^{p}\left(u_{0}, \ldots, u_{n+1}\right)}{t^{n}} \\
= & C \int \ldots \int_{F^{n+2}} \mathcal{K}^{p}\left(u_{0}, \ldots, u_{n+1}\right) \int_{F} \int_{0}^{\infty} \chi_{\mathcal{D}_{k_{2}}\left(u_{0}, \ldots, u_{n+1}\right)}(x, t) \frac{1}{t^{n}} \frac{\mathrm{d} t}{t} \mathrm{~d} \mu(x) \\
\mathrm{V} & \left(2 k_{2}\right)^{n}\left(\ln \left(2 u_{0}^{2}\right)\right)^{\frac{p}{2}} C C_{0} \int \ldots \int_{F^{n+2}} \mathcal{K}^{p}\left(u_{0}, \ldots, u_{n+1}\right) \mathrm{d} \mu\left(u_{0}\right) \ldots \mathrm{d} \mu\left(u_{n+1}\right) \frac{\mathrm{d} t}{t} \mathrm{~d} \mu\left(u_{n+1}\right) \\
= & C\left(N, n, \mathcal{K}, p, C_{0}, k, k_{0}, \lambda\right) \mathcal{M}_{\mathcal{K}^{p}(\mu) .} .
\end{aligned}
$$

Corollary 5.8. Let $0<\lambda<2^{n}, k>2, k_{0} \geq 1$ and $\mathcal{K}^{p}$ be some symmetric $\mu$-proper integrand (see Definition 4.4). There exists a constant $C=C\left(N, n, \mathcal{K}, p, C_{0}, k, k_{0}, \lambda\right)$ such that

$$
\iint_{0}^{\infty} \beta_{1 ; k}(x, t)^{p} \mathbb{1}_{\left\{\tilde{\delta}_{k_{0}}(B(x, t)) \geq \lambda\right\}} \frac{\mathrm{d} t}{t} \mathrm{~d} \mu(x) \leq C \mathcal{M}_{\mathcal{K}^{p}}(\mu) .
$$


Proof. Let $x \in \mathbb{R}^{N}$ and $t \in(0, \infty)$. With Hölder's inequality and (B) (see page 45), we get

$$
\begin{aligned}
\beta_{1 ; k}(x, t) & =\inf _{P \in \mathcal{P}(N, n)} \beta_{1 ; k}^{P}(x, t) \\
& =\inf _{P \in \mathcal{P}(N, n)} \frac{1}{t^{n}} \int_{B(x, k t)} \frac{d(y, P)}{t} \mathrm{~d} \mu(y) \\
& \leq \inf _{P \in \mathcal{P}(N, n)} \frac{1}{t^{n}}\left(\int_{B(x, k t)}\left(\frac{d(y, P)}{t}\right)^{p} \mathrm{~d} \mu(y)\right)^{\frac{1}{p}}\left(\int_{B(x, k t)} 1^{\frac{p}{p-1}} \mathrm{~d} \mu(y)\right)^{\frac{p-1}{p}} \\
& =\inf _{P \in \mathcal{P}(N, n)}\left(\frac{1}{t^{n}} \int_{B(x, k t)}\left(\frac{d(y, P)}{t}\right)^{p} \mathrm{~d} \mu(y)\right)^{\frac{1}{p}}\left(\frac{\mu(B(x, k t))}{t^{n}}\right)^{\frac{p-1}{p}} \\
& \stackrel{(\mathrm{B})}{\leq} \beta_{p ; k}(x, t)\left(C_{0}(2 k)^{n}\right)^{\frac{p-1}{p}} \\
& =C\left(n, p, C_{0}, k\right) \beta_{p ; k}(x, t) .
\end{aligned}
$$

Together with the previous theorem, the assertion holds.

\section{$5.3 \beta$-numbers, approximating planes and angles}

The following lemma states, that if two balls are close to each other and if each part of the support of $\mu$ contained in those balls is well approximated by some plane, then these planes have a small angle.

Lemma 5.9. Let $x, y \in F, c \geq 1, \xi \geq 1$ and $t_{x}, t_{y}>0$ with $c^{-1} t_{y} \leq t_{x} \leq$ ct $t_{y}$. Furthermore, let $k \geq 4 c$ and $0<\lambda<2^{n}$ with $\delta\left(B\left(x, t_{x}\right)\right) \geq \lambda, \delta\left(B\left(y, t_{y}\right)\right) \geq \lambda$ and $d(x, y) \leq \frac{k}{2 c} t_{x}$. Then there exists some constants $C_{3}=C_{3}\left(N, n, C_{0}, \lambda, \xi, c\right)>1$ and $\varepsilon_{0}=\varepsilon_{0}\left(N, n, C_{0}, \lambda, \xi, c\right)>0$ so that for all $\varepsilon<\varepsilon_{0}$ and all planes $P_{1}, P_{2} \in \mathcal{P}(N, n)$ with

$$
\beta_{1 ; k}^{P_{1}}\left(x, t_{x}\right) \leq \xi \varepsilon \quad \text { and } \quad \beta_{1 ; k}^{P_{2}}\left(y, t_{y}\right) \leq \xi \varepsilon
$$

we get:

(i) For all $w \in P_{1}$, we have $d\left(w, P_{2}\right) \leq C_{3} \varepsilon\left(t_{x}+d(w, x)\right)$ and, for all $w \in P_{2}$, we have $d\left(w, P_{1}\right) \leq C_{3} \varepsilon\left(t_{x}+d(w, x)\right)$,

(ii) $\Varangle\left(P_{1}, P_{2}\right) \leq C_{3} \varepsilon$.

Proof. Due to $\delta\left(B\left(x, t_{x}\right)\right) \geq \lambda$ and Corollary 5.3, there exist some constants $C_{1}>3$ and $C_{2}$ depending on $N, n, C_{0}, \lambda$, and some simplex $T=\Delta\left(x_{0}, \ldots, x_{n}\right) \in F \cap B\left(x, t_{x}\right)$ so that

(i) $T$ is an $\left(n, 10 n \frac{t_{x}}{C_{1}}\right)$-simplex,

(ii) $\mu\left(B\left(x_{i}, \frac{t_{x}}{C_{1}}\right) \cap B\left(x, t_{x}\right)\right) \geq \frac{t_{x}^{n}}{C_{2}} \quad$ for all $i \in\{0, \ldots, n\}$.

For $B_{i}:=B\left(x_{i}, \frac{t_{x}}{C_{1}}\right)$ and $i \in\{0, \ldots, n\}$, we have

$$
\mu\left(B_{i}\right) \geq \mu\left(B_{i} \cap B\left(x, t_{x}\right)\right) \geq \frac{t_{x}^{n}}{C_{2}} \geq \frac{t_{y}^{n}}{c^{n} C_{2}} .
$$


With (ii), we get $B_{i} \cap B\left(x, t_{x}\right) \neq \emptyset$ so that with $k \geq 4 c \geq 4$

$$
B_{i} \subset B\left(x, t_{x}+2 \frac{t_{x}}{C_{1}}\right) \subset B\left(x, k t_{x}\right)
$$

and

$$
B_{i} \subset B\left(y, t_{x}+2 \frac{t_{x}}{C_{1}}+\frac{k}{2 c} t_{x}\right) \subset B\left(y, c\left(2+\frac{k}{2 c}\right) t_{y}\right) \subset B\left(y, k t_{y}\right) .
$$

Now we see for $i \in\{0, \ldots, n\}$

$$
\begin{aligned}
& \frac{1}{\mu\left(B_{i}\right)} \int_{B_{i}} d\left(z, P_{1}\right)+d\left(z, P_{2}\right) \mathrm{d} \mu(z) \\
= & \frac{1}{\mu\left(B_{i}\right)} \int_{B_{i}} d\left(z, P_{1}\right) \mathrm{d} \mu(z)+\frac{1}{\mu\left(B_{i}\right)} \int_{B_{i}} d\left(z, P_{2}\right) \mathrm{d} \mu(z) \\
\stackrel{(5.22)(5.23)}{\leq} & C_{2} t_{x} \quad \frac{1}{t_{x}^{n}} \int_{B\left(x, k t_{x}\right)} \frac{d\left(z, P_{1}\right)}{t_{x}} \mathrm{~d} \mu(z) \\
& +c^{n} C_{2} t_{y} \frac{1}{t_{y}^{n}} \int_{B\left(y, k t_{y}\right)} \frac{d\left(z, P_{2}\right)}{t_{y}} \mathrm{~d} \mu(z) \\
= & C_{2} t_{x} \beta_{1 ; k}^{P_{1}}\left(x, t_{x}\right)+c^{n} C_{2} t_{y} \beta_{1 ; k}^{P_{2}}\left(y, t_{y}\right) \\
\leq & \left(1+c^{n+1}\right) C_{2} \xi t_{x} \varepsilon .
\end{aligned}
$$

With Chebyshev's inequality, there exists $z_{i} \in B_{i}$ so that

$$
d\left(z_{i}, P_{j}\right) \leq d\left(z_{i}, P_{1}\right)+d\left(z_{i}, P_{2}\right) \leq\left(1+c^{n+1}\right) C_{2} \xi t_{x} \varepsilon
$$

for $i \in\{0, \ldots, n\}$ and $j=1,2$. We set $y_{i}:=\pi_{P_{1}}\left(z_{i}\right)$ and with

$$
\varepsilon<\varepsilon_{0}:=\frac{1}{\left(1+c^{n+1}\right) C_{2} \xi} \min \left\{\frac{1}{C_{1}},\left(10\left(10^{n}+1\right) \frac{C_{1}}{6}\left(2 \frac{C_{1}}{3}\right)^{n}\right)^{-1}\right\}
$$

we deduce

$$
\begin{aligned}
d\left(y_{i}, x_{i}\right) & \leq d\left(y_{i}, z_{i}\right)+d\left(z_{i}, x_{i}\right) \\
& \leq d\left(z_{i}, P_{1}\right)+\frac{t_{x}}{C_{1}} \\
& \leq\left(1+c^{n+1}\right) C_{2} \xi t_{x} \varepsilon+\frac{t_{x}}{C_{1}} \leq 2 \frac{t_{x}}{C_{1}}
\end{aligned}
$$

so, with Lemma $2.14, S:=\Delta\left(y_{0}, \ldots, y_{n}\right)$ is an $\left(n, 6 n \frac{t_{x}}{C_{1}}\right)$-simplex and $S \subset B\left(x, \frac{2 t_{x}}{C_{1}}+t_{x}\right) \subset$ $B\left(x, 2 t_{x}\right)$. Furthermore, we have

$$
d\left(y_{i}, P_{2}\right) \leq \underbrace{d\left(y_{i}, z_{i}\right)}_{d\left(z_{i}, P_{1}\right)}+d\left(z_{i}, P_{2}\right) \stackrel{(5.24)}{\leq}\left(1+c^{n+1}\right) C_{2} \xi t_{x} \varepsilon .
$$


Now, with Lemma $2.28\left(C=\frac{C_{1}}{6 n}, \hat{C}=2, t=t_{x}, \sigma=\left(1+c^{n+1}\right) C_{2} \xi \varepsilon, m=n\right)$ we obtain

$$
\Varangle\left(P_{1}, P_{2}\right) \leq 4 n\left(10^{n}+1\right) \frac{C_{1}}{6}\left(2 \frac{C_{1}}{3}\right)^{n}\left(1+c^{n+1}\right) C_{2} \xi \varepsilon=C\left(N, n, C_{0}, \lambda, \xi, c\right) \varepsilon .
$$

Moreover, we have

$$
d\left(y_{0}, \pi_{P_{2}}\left(z_{0}\right)\right) \leq d\left(z_{0}, P_{1}\right)+d\left(z_{0}, P_{2}\right) \stackrel{(5.24)}{\leq}\left(1+c^{n+1}\right) C_{2} \xi t_{x} \varepsilon,
$$

so finally, with Lemma $2.29\left(\sigma=C \varepsilon, t=t_{x}, p_{1}=y_{0} \cdot p_{2}=\pi_{P_{2}}\left(z_{0}\right)\right)$, we get for $w \in P_{1}$

$$
\begin{aligned}
d\left(w, P_{2}\right) & \leq C\left(d\left(w, y_{0}\right)+t_{x}\right) \varepsilon \\
& \leq C\left(d(w, x)+t_{x}\right) \varepsilon
\end{aligned}
$$

and for $w \in P_{2}$

$$
\begin{aligned}
d\left(w, P_{1}\right) & \leq C\left(d\left(w, \pi_{P_{2}}\left(z_{0}\right)\right)+t_{x}\right) \\
& \leq C\left(d(w, x)+t_{x}\right) \varepsilon
\end{aligned}
$$

where $C=C\left(N, n, C_{0}, \lambda, \xi, c\right)$.

The next lemma describes the distance from a plane to a ball if the plain approximates the support of $\mu$ contained in the ball.

Lemma 5.10. Let $\sigma>0, x \in F, t>0$ and $\lambda>0$ with $\delta(B(x, t)) \geq \lambda$. If $P \in \mathcal{P}(N, n)$ with $\beta_{1 ; k}^{P}(x, t) \leq \sigma$, we have:

(i) There exists some $y \in B(x, t) \cap F$ so that $d(y, P) \leq \frac{t}{\lambda} \sigma$.

(ii) If additionally $\sigma \leq \lambda$, we have $B(x, 2 t) \cap P \neq \emptyset$.

Proof. With the requirements, we get $\mu(B(x, t)) \geq t^{n} \lambda$, and so

$$
\frac{1}{\mu(B(x, t))} \int_{B(x, t)} d(z, P) \mathrm{d} \mu(z) \leq \frac{t}{\lambda} \frac{1}{t^{n}} \int_{B(x, k t)} \frac{d(z, P)}{t} \mathrm{~d} \mu(z)=\frac{t}{\lambda} \beta_{1 ; k}^{P}(x, t) \leq \frac{t}{\lambda} \sigma .
$$

With Chebyshev's inequality, we get some $y \in B(x, t) \cap F$ with $d(y, P) \leq \frac{t}{\lambda} \sigma$. If $\sigma \leq \lambda$, it follows that $B(x, 2 t) \cap P \neq \emptyset$. 



\section{Construction of the Lipschitz graph}

Our aim is to prove Theorem 4.6. We have to cover a major part of the support of the measure $\mu$ by some Lipschitz graph $\Gamma$. To get $\Gamma$, we construct some Lipschitz function where the graph of this function gives $\Gamma$. The Lipschitz function will be defined on some $n$-dimensional plane $P_{0}$ which approximates the support of the measure $\mu$ in the sense of $\beta$-numbers. The existence of such a plane $P_{0}$ is assumed in the following theorem, which is quite similar to Theorem 4.6.

Theorem 6.1. Let $\mathcal{K}:\left(\mathbb{R}^{N}\right)^{n+2} \rightarrow[0, \infty)$ and $p=2$. There exists some $k>2$ such that for every $C_{0} \geq 10$, there exists some $\eta=\eta\left(N, n, \mathcal{K}, C_{0}, k\right) \in\left(0,2^{-(n+1)}\right]$ so that if $\mu$ is a Borel measure on $\mathbb{R}^{N}$ with compact support $F$ such that $\mathcal{K}^{2}$ is a symmetric $\mu$-proper integrand (cf. Definition 4.4) and $\mu$ fulfils

(A) $\mu(B(0,5)) \geq 1, \mu\left(\mathbb{R}^{N} \backslash B(0,5)\right)=0$,

(B) $\mu(B) \leq C_{0}(\operatorname{diam} B)^{n}$ for every ball $B$,

(C) $\mathcal{M}_{\mathcal{K}^{2}}(\mu) \leq \eta$,

(D) $\beta_{1 ; k ; \mu}^{P_{0}}(0,5) \leq \eta$ for some plane $P_{0} \in \mathcal{P}(N, n)$ with $0 \in P_{0}$,

then there exists some Lipschitz function $A: P_{0} \rightarrow P_{0}^{\perp} \subset \mathbb{R}^{N}$ so that the graph $G(A) \subset \mathbb{R}^{N}$ fulfils

$$
\mu(G(A)) \geq \frac{99}{100} \mu\left(\mathbb{R}^{N}\right) .
$$

$P_{0}^{\perp}:=\left\{x \in \mathbb{R}^{N} \mid x \cdot v=0\right.$ for all $\left.v \in P_{0}\right\}$ denotes the orthogonal complement of $P_{0}$.

At first, we see that, under the assumption that this theorem is correct, we can prove Theorem 4.6. The remaining proof of Theorem 6.1 is then given by the rest of this chapter 6 together with the following chapters 7 and 8 .

Proof of Theorem 4.6. Let $\mathcal{K}^{2}$ be some $\mu$-proper integrand and let $C_{0}>0$. Using Lemma 4.1, we can assume that $\mathcal{K}$ is symmetric. Furthermore, let $k>2$ and $0<\eta \leq 2^{-(n+1)}$ be the constants given by Theorem 6.1.

Now let $\tilde{\mu}$ be a measure that fulfils the requirements of Theorem 4.6, that means $\tilde{\mu}$ is a Borel measure with a compact support $\tilde{F}$ and
$(\tilde{\mathrm{A}}) \tilde{\mu}(B(0,2)) \geq 1, \tilde{\mu}\left(\mathbb{R}^{N} \backslash B(0,2)\right)=0$,

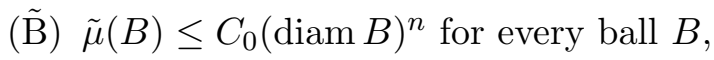
$(\tilde{\mathrm{C}}) \mathcal{M}_{\mathcal{K}^{2}}(\tilde{\mu}) \leq \frac{\eta^{2}}{\hat{C}}$ 
where $\hat{C}=\hat{C}\left(N, n, \mathcal{K}, C_{0}, k\right) \geq 1$ is independent of $\tilde{\mu}$ and given by the following estimate (6.1). We have $\tilde{F} \subset B(0,2)$, as $\mathbb{R}^{N} \backslash B(0,2)$ is a $\tilde{\mu}$-null set. We have

$$
\delta_{\tilde{\mu}}(B(0,2))=\frac{\tilde{\mu}(B(0,2))}{2^{n}} \stackrel{(\tilde{A})}{\geq} \frac{1}{2^{n}} .
$$

So the requirements of Theorem 5.6 with $x=y=0, t=2, \lambda=\frac{1}{2^{n}}$ and $k_{0}=1$ are fulfilled and using Hölder's inequality (see the proof of Corollary 5.8, page 59), we obtain

$$
\beta_{1 ; k ; \tilde{\mu}}(0,2)^{2} \leq C\left(n, C_{0}, k\right) \beta_{2 ; k ; \tilde{\mu}}(0,2)^{2} \leq \hat{C} \frac{\mathcal{M}_{\mathcal{K}^{2} ; k_{1} ; \tilde{\mu}}(0,2)}{2^{n}}<\hat{C} \mathcal{M}_{\mathcal{K}^{2}}(\tilde{\mu}) \stackrel{(\tilde{\mathrm{C}})}{\leq} \eta^{2} .
$$

So there exists some $n$-dimensional plane $\tilde{P}_{0} \in \mathcal{P}(N, n)$ with $\beta_{1 ; k ; \tilde{\mu}}^{\tilde{P}_{0}}(0,2) \leq \eta$. Using Lemma 5.10 (i) with $\sigma=\eta, x=0, t=2, \lambda=\frac{1}{2^{n}}$ and $P=\tilde{P}_{0}$, there exists some $y \in B(0,2) \cap \tilde{F}$ such that $\left|\pi_{\tilde{P}_{0}}(0)\right|=d\left(0, \tilde{P}_{0}\right) \leq d(0, y)+d\left(y, \tilde{P}_{0}\right) \leq 2+2^{n+1} \eta \leq 3$. Now we define a measure $\mu$ by $\mu(\cdot):=\tilde{\mu}\left(\cdot+\pi_{\tilde{P}_{0}}(0)\right)$. This is also a Borel measure with compact support $F:=\tilde{F}-\pi_{\tilde{P}_{0}}(0) \subset$ $B(0,5)$ and

(A) $\mu(B(0,5)) \geq 1, \mu\left(\mathbb{R}^{N} \backslash B(0,5)\right)=0$,

(B) $\mu(B) \leq C_{0}(\operatorname{diam} B)^{n}$ for every ball $B$,

(C) $\mathcal{M}_{\mathcal{K}^{2}}(\mu)=\mathcal{M}_{\mathcal{K}^{2}}(\tilde{\mu}) \stackrel{\tilde{\mathrm{C}}}{\leq} \frac{\eta^{2}}{\hat{C}} \leq \eta$,

(D) $\beta_{1 ; k ; \mu}^{P_{0}}(0,5)=\left(\frac{2}{5}\right)^{n+1} \beta_{1 ; k ; \tilde{\mu}}^{\tilde{P}_{0}}(0,2)<\eta$, where $P_{0}:=\tilde{P}_{0}-\pi_{\tilde{P}_{0}}(0)$ and $0 \in P_{0}$.

Hence we can apply Theorem 6.1 and obtain some Lipschitz function $A: P_{0} \rightarrow P_{0}^{\perp}$ so that the graph $G(A)$ fulfils $\mu(G(A)) \geq \frac{99}{100} \mu\left(\mathbb{R}^{N}\right)$. We set $\Gamma:=G(A)+\pi_{\tilde{P}_{0}}(0)$ and get

$$
\tilde{\mu}(\Gamma)=\mu(G(A)) \geq \frac{99}{100} \mu\left(\mathbb{R}^{N}\right)=\frac{99}{100} \tilde{\mu}\left(\mathbb{R}^{N}\right) .
$$

\subsection{Partition of the support of the measure $\mu$}

Now we start with the proof of Theorem 6.1. Let $\mathcal{K}:\left(\mathbb{R}^{N}\right)^{n+2} \rightarrow[0, \infty)$ and let $C_{0} \geq 10$ be some fixed constant. There is one step in the proof which only works for integrability exponent $p=2$. $(p=2$ is used in Lemma 8.11 so that the results of Theorem 7.3 and Theorem 7.17 fit together.) Since most of the proof can be given with less constraints to $p$, we start with $p \in(1, \infty)$ and restrict to $p=2$ only if needed. Furthermore, let $k>2,0<\eta \leq 2^{-(n+1)}$, $P_{0} \in \mathcal{P}(N, n)$ with $0 \in P_{0}$ and $\mu$ be a Borel measure on $\mathbb{R}^{N}$ with compact support $F$ such that $\mathcal{K}^{p}$ is a symmetric $\mu$-proper integrand (cf. Definition 4.4) and
(A) $\mu(B(0,5)) \geq 1, \mu\left(\mathbb{R}^{N} \backslash B(0,5)\right)=0$,
(B) $\mu(B) \leq C_{0}(\operatorname{diam} B)^{n}$ for every ball $B$, 
(C) $\mathcal{M}_{\mathcal{K}^{p}}(\mu) \leq \eta$,

(D) $\beta_{1 ; k ; \mu}^{P_{0}}(0,5) \leq \eta$.

In this chapter, we will prove that if $k$ is large and $\eta$ is small enough, we can construct some function $A: P_{0} \rightarrow P_{0}^{\perp}$ which covers some part of the support $F$ of $\mu$. For this purpose, we will give a partition of the support of $\mu$ in four parts, $\operatorname{supp}(\mu)=\mathcal{Z} \dot{\cup} F_{1} \dot{\cup} F_{2} \dot{\cup} F_{3}$, and construct the function $A$ so that the graph of $A$ covers $\mathcal{Z}$, i.e., $\mathcal{Z} \subset G(A)$.

The following chapters 7 and 8 will give a proof of

$$
\mu\left(F_{1} \cup F_{2} \cup F_{3}\right) \leq \frac{1}{100} \stackrel{(\mathrm{A})}{\leq} \frac{1}{100} \mu\left(\mathbb{R}^{N}\right),
$$

hence we will obtain

$$
\mu(G(A)) \geq \mu(\mathcal{Z}) \geq \frac{99}{100} \mu\left(\mathbb{R}^{N}\right),
$$

which is the statement of the proof of Theorem 6.1.

From now on, we will only work with the fixed measure $\mu$, so we can simplify the expressions by setting $\beta_{1 ; k}:=\beta_{1 ; k ; \mu}$ and $\delta(\cdot):=\delta_{\mu}(\cdot)$. Furthermore, we fix the constant

$$
\delta:=\min \left\{\frac{10^{-10}}{600^{n} N_{0}}, \frac{2}{50^{n}}\right\},
$$

where $N_{0}=N_{0}(N)$ is the constant from Besicovitch's Covering Theorem A.12.

Definition 6.2. Let $\alpha, \varepsilon>0$. We define the set

$$
S_{\text {total }}^{\varepsilon, \alpha}:=\left\{\begin{array}{l|ll}
(x, t) \in F \times(0,50) & \begin{array}{ll}
(i) & \delta(B(x, t)) \geq \frac{1}{2} \delta \\
(i i) & \beta_{1 ; k}(x, t)<2 \varepsilon
\end{array} \\
(\text { iii }) & \exists P_{(x, t)} \in \mathcal{P}(N, n) \text { s.t. }\left\{\begin{array}{c}
\beta_{1 ; k}^{P_{(x, t)}}(x, t) \leq 2 \varepsilon \\
\text { and } \\
\Varangle\left(P_{(x, t)}, P_{0}\right) \leq \alpha
\end{array}\right.
\end{array}\right\} .
$$

Having in mind that the definition of $S_{\text {total }}^{\varepsilon, \alpha}$ depends on the choice of $\varepsilon$ and $\alpha$, we will normally skip these and write $S_{\text {total }}$ instead. In the same manner, we will handle the following definitions of $H, h$ and $S$. For $x \in F$ we define

$$
H(x):=\left\{t \in(0,50) \mid \exists y \in F, \exists \tau \text { with } \frac{t}{4} \leq \tau \leq \frac{t}{3}, d(x, y)<\frac{\tau}{3} \text { and }(y, \tau) \notin S_{\text {total }}\right\}
$$

and

$$
h(x):=\sup (H(x) \cup\{0\})
$$

and set

$$
S:=\left\{(x, t) \in S_{\text {total }} \mid t \geq h(x)\right\} .
$$

Lemma 6.3. Let $\alpha, \varepsilon>0$. If $\eta \leq 2 \varepsilon$, we have

(i) $F \times[10,50) \subset S_{\text {total }}$, 
(ii) $H(x) \subset(0,40)$ for all $x \in F$,

(iii) $h(x) \leq 40$ for all $x \in F$,

(iv) $F \times[40,50) \subset\{(x, t) \in F \times(0,50) \mid t \geq h(x)\}=S$,

(v) If $(x, t) \in S$ and $t \leq t^{\prime}<50$, we have $\left(x, t^{\prime}\right) \in S$.

(vi) For every $(x, t) \in S_{\text {total }}$, there exists some plane $P_{(x, t)}$ with $\beta_{1 ; k}^{P_{(x, t)}}(x, t) \leq 2 \varepsilon$ and $\Varangle\left(P_{(x, t)}, P_{0}\right) \leq \alpha$.

Proof. (i) Let $x \in F \stackrel{(A)}{\subset} B(0,5)$ and $10 \leq t<50$, which implies $F \subset B(0,5) \subset B(x, t)$. We obtain

$$
\begin{aligned}
\delta(B(x, t)) & =\frac{\mu(B(x, t))}{t^{n}} \stackrel{(\mathrm{A})}{>} \frac{1}{50^{n}} \stackrel{(6.2)}{=} \frac{\delta}{2}, \\
\beta_{1 ; k}(x, t) & \leq \beta_{1 ; k}^{P_{0}}(x, t)=\left(\frac{5}{t}\right)^{n+1} \quad \beta_{1 ; k}^{P_{0}}(0,5) \stackrel{(\mathrm{D})}{<} \eta \leq 2 \varepsilon .
\end{aligned}
$$

Set $P_{(x, 10)}:=P_{0}$ and we get $(x, t) \in S_{\text {total }}$, which implies that $F \times[10,50) \subset S_{\text {total }}$.

(ii) Let $x \in F$ and $t \in[40,50)$. For arbitrary $y \in F$ and $\tau \in\left[\frac{t}{4}, \frac{t}{3}\right]$, we get $\tau \geq 10$ and using (i), we obtain $(y, \tau) \in S_{\text {total }}$, which implies that $H(x) \subset(0,40)$.

(iii) The statement follows directly from (ii).

(iv) The inclusion follows directly from (iii). For the equality it is enough to prove that the central set is contained in $S$. Let $x \in F$ and $t \in(0,50)$ with $h(x) \leq t<50$. Assume that $(x, t) \notin S$. Due to $h(x) \leq t$, we obtain $(x, t) \notin S_{\text {total }}$, which implies with (i) that $t<10$. Hence we have $3 t \in(0,50)$ and

$$
x \in F, \frac{3 t}{4} \leq t \leq \frac{3 t}{3}, d(x, x)<\frac{t}{3} \text { and }(x, t) \notin S_{\text {total }},
$$

which implies that $3 t \in H(x)$. We get $h(x) \geq 3 t>t$. This is in contradiction to $t \geq h(x)$, so we obtain $(x, t) \in S$.

(v) Let $(x, t) \in S$ and $t \leq t^{\prime}<50$.

We have $x \in F$ and $h(x) \leq t \leq t^{\prime}<50$ so with (iv) we conclude that $\left(x, t^{\prime}\right) \in S$.

(vi) The existence of such a plane is guaranteed by the definition of $S_{\text {total }}$.

Remember that the function $h$ depends on the set $S_{\text {total }}$, which depends on the choice of $\varepsilon$ and $\alpha$. Hence the sets defined in the following definition depend on $\alpha$ and $\varepsilon$ as well.

Definition 6.4 (Partition of $F$ ). Let $\alpha, \varepsilon>0$. We define

$$
\mathcal{Z}:=\{x \in F \mid h(x)=0\},
$$




$$
\begin{aligned}
& F_{1}:=\left\{\begin{array}{l|l}
x \in F \backslash \mathcal{Z} \mid \begin{array}{l}
\exists y \in F, \exists \tau \in\left[\frac{h(x)}{5}, \frac{h(x)}{2}\right], \text { with } d(x, y) \leq \frac{\tau}{2} \\
\text { and } \\
\delta(B(y, \tau)) \leq \delta
\end{array}
\end{array}\right\}, \\
& F_{2}:=\left\{\begin{array}{l|l}
x \in F \backslash\left(\mathcal{Z} \cup F_{1}\right) \mid \begin{array}{l}
\exists y \in F, \exists \tau \in\left[\frac{h(x)}{5}, \frac{h(x)}{2}\right], \text { with } d(x, y) \leq \frac{\tau}{2} \\
\text { and } \\
\beta_{1 ; k}(y, \tau) \geq \varepsilon
\end{array}
\end{array}\right\}, \\
& F_{3}:=\left\{\begin{array}{l|l}
x \in F \backslash\left(\mathcal{Z} \cup F_{1} \cup F_{2}\right) & \begin{array}{l}
\exists y \in F, \exists \tau \in\left[\frac{h(x)}{5}, \frac{h(x)}{2}\right], \text { with } d(x, y) \leq \frac{\tau}{2} \\
\text { and for all planes } P \in \mathcal{P}(N, n) \text { with } \\
\beta_{1 ; k}^{P}(y, \tau) \leq \varepsilon \text { we have } \Varangle\left(P, P_{0}\right) \geq \frac{3}{4} \alpha
\end{array}
\end{array}\right\} .
\end{aligned}
$$

In this chapter, we prove that $\mathcal{Z}$ is rectifiable by constructing a function $A$ such that the graph of $A$ will cover $\mathcal{Z}$. This is done by inverting the orthogonal projection $\left.\pi\right|_{\mathcal{Z}}: \mathcal{Z} \rightarrow P_{0}$. After that, to complete the proof, it remains to show that $\mathcal{Z}$ constitutes the major part of $F$. Right now, we can prove that $\mu\left(F_{2}\right) \leq 10^{-6}$ (cf. section $8.3, F_{2}$ is small) where the control of the other sets need some more preparations.

Lemma 6.5. Let $\alpha, \varepsilon>0$. Definition 6.4 gives a partition of $F$, that means

$$
F=\mathcal{Z} \dot{\cup} F_{1} \dot{\cup} F_{2} \dot{\cup} F_{3} .
$$

Proof. From the definition we see that the sets are disjoint. We show

$$
F \backslash \mathcal{Z} \subset F_{1} \cup F_{2} \cup F_{3}
$$

Let $x \in F \backslash \mathcal{Z}$, so we have $h(x)>0$. There exist some sequences $\left(y_{l}\right)_{l \in \mathbb{N}} \in F^{\mathbb{N}},\left(t_{l}\right)_{l \in \mathbb{N}}$ and $\left(\tau_{l}\right)_{l \in \mathbb{N}}$ so that for all $l \in \mathbb{N}$, we have $0<t_{l} \leq h(x), t_{l} \rightarrow h(x), \frac{t_{l}}{4} \leq \tau_{l} \leq \frac{t_{l}}{3}, d\left(x, y_{l}\right)<\frac{\tau_{l}}{3}$ and $\left(y_{l}, \tau_{l}\right) \notin S_{\text {total }}$. Due to $\tau_{l} \leq \frac{t_{l}}{3} \leq \frac{h(x)}{3} \leq \frac{50}{3}$, we have for every $l \in \mathbb{N}$ either

(a) $\delta\left(B\left(y_{l}, \tau_{l}\right)\right)=\frac{\mu\left(B\left(y_{l}, \tau_{l}\right)\right)}{\tau_{l}^{n}}<\frac{1}{2} \delta$ or

(b) $\delta\left(B\left(y_{l}, \tau_{l}\right)\right) \geq \frac{1}{2} \delta$ and $\beta_{1 ; k}\left(y_{l}, \tau_{l}\right) \geq 2 \varepsilon$ or

(c) $\delta\left(B\left(y_{l}, \tau_{l}\right)\right) \geq \frac{1}{2} \delta$ and $\beta_{1 ; k}\left(y_{l}, \tau_{l}\right)<2 \varepsilon$, and for every plane $P \in \mathcal{P}(N, n)$ with $\beta_{1 ; k}^{P}\left(y_{l}, \tau_{l}\right) \leq 2 \varepsilon$, we have $\Varangle\left(P, P_{0}\right)>\alpha$.

Choose $l$ so large that $\frac{4 h(x)}{5} \leq t_{l}$. We obtain

$$
\frac{h(x)}{5} \leq \frac{t_{l}}{4} \leq \tau_{l} \leq \frac{t_{l}}{3} \leq \frac{h(x)}{2}
$$

Furthermore, we have $y_{l} \in F$ and $d\left(x, y_{l}\right) \leq \frac{\tau_{l}}{3}<\frac{\tau_{l}}{2}$. Since $\left(y_{l}, \tau_{l}\right)$ fulfils either case (a) or case (b) or case (c), it follows $x \in F_{1} \cup F_{2} \cup F_{3}$.

The following lemma is for later use (cf. Lemma 8.10 and Lemma 8.11). 
Lemma 6.6. Let $\alpha>0$. There exists some constant $\bar{\varepsilon}=\bar{\varepsilon}\left(N, n, C_{0}, \alpha\right)$ so that if $\eta<2 \bar{\varepsilon}$ and $k \geq 400$, there holds for all $\varepsilon \in\left[\frac{\eta}{2}, \bar{\varepsilon}\right)$ :

If $x \in F_{3}$ and $h(x) \leq t \leq \min \{100 h(x), 49\}$, we get $\Varangle\left(P_{(x, t)}, P_{0}\right)>\frac{1}{2} \alpha$, where $P_{(x, t)}$ is the plane granted since $(x, t) \in S_{\text {total }}$ (cf. Lemma 6.3 (vi)).

Proof. Let $\alpha>0$ and $k \geq 400$. We set $\bar{\varepsilon}:=\min \left\{\varepsilon_{0}, \varepsilon_{0}^{\prime}, \alpha\left(4 C_{3}+4 C_{3}^{\prime}\right)^{-1}\right\}$, where $\varepsilon_{0}, \varepsilon_{0}^{\prime}, C_{3}$ and $C_{3}^{\prime}$ depend only on $N, n$ and $C_{0}$ will be chosen during this proof. Furthermore, let $\eta \leq 2 \varepsilon<2 \bar{\varepsilon}$. Due to $x \in F_{3}$, there exists some $y \in F$ and $\tau \in\left[\frac{h(x)}{5}, \frac{h(x)}{2}\right]$ with $d(x, y) \leq \frac{\tau}{2}$ so that for all planes $P \in \mathcal{P}(N, n)$ with $\beta_{1 ; k}^{P}(y, \tau) \leq \varepsilon$, we have $\Varangle\left(P, P_{0}\right) \geq \frac{3}{4} \alpha$. Furthermore, with Lemma 6.3 (iv), we deduce $(x, h(x)) \in S \subset S_{\text {total }}$ and so $\beta_{1 ; k}^{P_{(x, h(x))}}(x, h(x)) \leq 2 \varepsilon$. For $z \in B(y, \tau)$, we get

$$
d(x, z) \leq d(x, y)+d(y, z) \leq \frac{\tau}{2}+\tau<2 \tau \leq 2 \frac{h(x)}{2}=h(x),
$$

which implies $B(y, \tau) \subset B(x, h(x))$. Using this inclusion and $\tau \geq \frac{h(x)}{5}$, we deduce

$$
\begin{aligned}
\beta_{1 ; k}^{P_{(x, h(x))}}(y, \tau) & \leq\left(\frac{h(x)}{\tau}\right)^{n+1} \frac{1}{h(x)^{n}} \int_{B(x, k h(x))} \frac{d\left(y, P_{(x, h(x))}\right)}{h(x)} \mathrm{d} \mu(y) \\
& \leq 5^{n+1} \beta_{1 ; k}^{P_{(x, h(x))}}(x, h(x)) \\
& \leq 5^{n+1} 2 \varepsilon .
\end{aligned}
$$

Since $x \in F_{3}$, we have $x \notin\left(\mathcal{Z} \cup F_{1} \cup F_{2}\right)$ and so $\beta_{1 ; k}(y, \tau)<\varepsilon$. Hence there exists some plane $P^{\prime} \in \mathcal{P}(N, n)$ with $\beta_{1 ; k}^{P^{\prime}}(y, \tau)<\varepsilon$ and by definition of $F_{3}$ we get

$$
\Varangle\left(P^{\prime}, P_{0}\right) \geq \frac{3}{4} \alpha .
$$

Moreover, with $x \notin F_{1}$, we have $\delta(B(y, \tau))>\delta$ and with Lemma 5.9 (ii) ( $x=y, t_{x}=t_{y}=\tau$, $\left.\lambda=\delta=\delta(N, n), \xi=2 \cdot 5^{n+1}, c=1\right)$, there exist some constants $C_{3}=C_{3}\left(N, n, C_{0}\right)>1$ and $\varepsilon_{0}=\varepsilon_{0}\left(N, n, C_{0}\right)>0$ so that

$$
\Varangle\left(P^{\prime}, P_{(x, h(x))}\right) \leq C_{3} \varepsilon .
$$

We assumed $h(x) \leq t \leq \min \{100 h(x), 49\}$ so, with Lemma 6.3 (iv), we get $(x, t) \in S$. Hence

$$
\beta_{1 ; k}^{P_{(x, t)}}(x, h(x)) \leq\left(\frac{t}{h(x)}\right)^{n+1} \beta_{1 ; k}^{P_{(x, t)}}(x, t) \leq 2 \cdot 100^{n+1} \varepsilon .
$$

and again, with Lemma 5.9 (ii) ( $\left.x=y, t_{x}=h(x), t_{y}=t, \lambda=\frac{\delta}{2}, \xi=2 \cdot 100^{n+1}, c=100\right)$, there exist some constants $C_{3}^{\prime}=C_{3}^{\prime}\left(N, n, C_{0}\right)>1$ and $\varepsilon_{0}^{\prime}=\varepsilon_{0}^{\prime}\left(N, n, C_{0}\right)>0$ so that

$$
\Varangle\left(P_{(x, h(x))}, P_{(x, t)}\right) \leq C_{3}^{\prime} \varepsilon .
$$

Finally, we obtain with Lemma 2.20 (triangle inequality for $\Varangle(\cdot, \cdot))$ and using $\varepsilon<\frac{\alpha}{4\left(C_{3}+C_{3}^{\prime}\right)}$

$$
\Varangle\left(P_{(x, t)}, P_{0}\right) \geq \Varangle\left(P^{\prime}, P_{0}\right)-\Varangle\left(P^{\prime}, P_{(x, h(x))}\right)-\Varangle\left(P_{(x, h(x))}, P_{(x, t)}\right) \geq \frac{3}{4} \alpha-\left(C_{3}+C_{3}^{\prime}\right) \varepsilon>\frac{1}{2} \alpha .
$$




\subsection{The distance to a well approximable ball}

We recall that the set $S$ depends on the choice of $\alpha$ and $\varepsilon$. Hence the functions $d$ and $D$ defined in the next definition depend on $\alpha$ and $\varepsilon$ as well. We introduce $\pi:=\pi_{P_{0}}: \mathbb{R}^{N} \rightarrow P_{0}$, the orthogonal projection on $P_{0}$.

Definition 6.7 (The functions $d$ and $D$ ). Let $\alpha, \varepsilon>0$. If $\eta \leq 2 \varepsilon$, we get with Lemma 6.3 (iv) that $S \neq \emptyset$. We define $d: \mathbb{R}^{N} \rightarrow[0, \infty)$ with

$$
d(x):=\inf _{(X, t) \in S}(d(X, x)+t)
$$

and $D: P_{0} \rightarrow[0, \infty)$ with

$$
D(y):=\inf _{x \in \pi^{-1}(y)} d(x) .
$$

Let us call a ball $B(X, t)$ with $(X, t) \in S$ a good ball. Then the function $d$ measures the distance from the given point $x$ to the nearest good ball, using the furthermost point in the ball. This implies that a ball $B(x, d(x))$ always contains some good ball. The function $D$ does something similar. Consider the projection of all good balls to the plane $P_{0}$. Then $D$ measures the distance to the nearest projected good ball in the same sense as above (cf. next lemma).

Lemma 6.8. Let $\alpha, \varepsilon>0$. If $\eta \leq 2 \varepsilon$, we have for $y \in P_{0}$ that

$$
D(y)=\inf _{(X, t) \in S}(d(\pi(X), y)+t) .
$$

Proof. We get

$$
\begin{aligned}
\inf _{x \in \pi^{-1}(y)} d(x) & =\inf _{x \in \pi^{-1}(y)} \inf _{(X, t) \in S}(d(X, x)+t) \\
& \geq \inf _{x \in \pi^{-1}(y)} \inf _{(X, t) \in S}(d(\pi(X), \pi(x))+t) \\
& =\inf _{(X, t) \in S}(d(\pi(X), y)+t) .
\end{aligned}
$$

Assume

$$
\inf _{x \in \pi^{-1}(y)} d(x)>\inf _{(X, t) \in S}(d(\pi(X), y)+t) .
$$

Let $\left(X_{l}, t_{l}\right) \in S$ be some sequence with

$$
\lim _{l \rightarrow \infty}\left(d\left(\pi\left(X_{l}\right), y\right)+t_{l}\right)=\inf _{(X, t) \in S}(d(\pi(X), y)+t) .
$$

Now there exists some $l \in \mathbb{N}$ so that

$$
\begin{aligned}
\inf _{x \in \pi^{-1}(y)} d(x) & >d\left(\pi\left(X_{l}\right), y\right)+t_{l} \\
& =d\left(\pi\left(X_{l}\right)+X_{l}-\pi\left(X_{l}\right), y+X_{l}-\pi\left(X_{l}\right)\right)+t_{l} \\
& =d(X_{l}, \underbrace{y+X_{l}-\pi\left(X_{l}\right)}_{\in \pi^{-1}(y)})+t_{l}
\end{aligned}
$$




$$
\begin{aligned}
& \geq \inf _{x \in \pi^{-1}(y)} d\left(X_{l}, x\right)+t_{l} \\
& \geq \inf _{x \in \pi^{-1}(y)} \inf _{(X, t) \in S}(d(X, x)+t) \\
& =\inf _{x \in \pi^{-1}(y)} d(x) .
\end{aligned}
$$

This is a contradiction.

Lemma 6.9. The functions $d$ and $D$ are Lipschitz functions with Lipschitz constant 1.

Proof. Let $x, y \in \mathbb{R}^{N}$. We get with the triangle inequality $d(x) \leq d(y)+d(x, y)$ and $d(y) \leq$ $d(x)+d(x, y)$. This implies $|d(x)-d(y)| \leq d(x, y)$. Using the previous lemma, we can use the same argument for the function $D$.

Lemma 6.10. We have $\left\{x \in \mathbb{R}^{N} \mid d(x)<1\right\} \subset B(0,6)$ and $d(x) \leq 60$ for all $x \in B(0,5)$.

Proof. Let $x \in \mathbb{R}^{N}$ with $\inf _{(X, t) \in S}(d(X, x)+t)=d(x)<1$. Hence there exists some $X \in F \subset$ $B(0,5)$ with $d(0, x) \leq d(0, X)+d(X, x)<6$.

Now let $x \in B(0,5)$. We have $d(x)=\inf _{(X, t) \in S}(d(X, x)+t) \leq 10+50=60$.

Lemma 6.11. Let $\alpha, \varepsilon>0$. If $\eta \leq 2 \varepsilon$, we have $d(x) \leq h(x)$ for all $x \in F$ and

$$
\mathcal{Z}=\{x \in F \mid d(x)=0\}, \quad \pi(\mathcal{Z})=\left\{y \in P_{0} \mid D(y)=0\right\} .
$$

Furthermore, both sets $\mathcal{Z}$ and $\pi(\mathcal{Z})$ are closed. We recall that $\pi$ denotes the orthogonal projection on the plane $P_{0}$.

Proof. Let $x \in F$. With Lemma 6.3 (iv), we have $(x, h(x)) \in S$ and hence

$$
d(x)=\inf _{(X, t) \in S}(d(X, x)+t) \stackrel{(x, h(x)) \in S}{\leq} d(x, x)+h(x)=h(x) .
$$

It follows

$$
h(x)=0 \Rightarrow d(x)=0
$$

and consequently $\mathcal{Z} \subset\{x \in F \mid d(x)=0\}$. Now we show

$$
h(x)>0 \Rightarrow d(x)>0
$$

and, all in all, $\mathcal{Z}=\{x \in F \mid d(x)=0\}$.

Let $x \in F$ with $h(x)>0$. There exist some sequences $\left(t_{l}\right)_{l \in \mathbb{N}},\left(\tau_{l}\right)_{l \in \mathbb{N}}$ and $\left(y_{l}\right)_{l \in \mathbb{N}}$ with $t_{l} \rightarrow h(x), \frac{t_{l}}{4} \leq \tau_{l} \leq \frac{t_{l}}{3}, d\left(x, y_{l}\right)<\frac{\tau_{l}}{3}$ and $\left(y_{l}, \tau_{l}\right) \notin S_{\text {total }}$ for all $l \in \mathbb{N}$. There exists some $M \in \mathbb{N}$ so that, for all $l \in \mathbb{N}$ with $l \geq M$, we obtain $\frac{h(x)}{5}<\tau_{l}$. Furthermore, there exists some sequence $\left(X_{i}, s_{i}\right)_{i \in \mathbb{N}} \in S^{\mathbb{N}}$ with $\lim _{i \rightarrow \infty} d\left(X_{i}, x\right)+s_{i}=d(x)$.

1. Case: There exists some subsequence with $d\left(X_{i_{j}}, x\right) \rightarrow 0$.

Choose another subsequence so that $d\left(X_{i_{j_{l}}}, x\right)<\frac{\tau_{l}}{3}-d\left(x, y_{l}\right)$ for all $l \geq M$. Now we obtain for all $l \geq M$ that $d\left(X_{i_{j_{l}}}, y_{l}\right) \leq d\left(X_{i_{j_{l}}}, x\right)+d\left(x, y_{l}\right)<\frac{\tau_{l}}{3}$ and, according to the definition of $h$, we deduce $h\left(X_{i_{j_{l}}}\right) \geq t_{l}$. Now we get with $\left(X_{i_{j_{l}}}, s_{i_{j_{l}}}\right) \in S$,

$$
d\left(X_{i_{j_{l}}}, x\right)+s_{i_{j_{l}}} \geq s_{i_{j_{l}}} \geq h\left(X_{i_{j_{l}}}\right) \geq t_{l} \geq 3 \tau_{l}>3 \frac{h(x)}{5}>0 .
$$


So we have $d(x)>0$.

2. Case: There exists some $c>0$ so that, for all $l \in \mathbb{N}$, we have $d\left(X_{l}, x\right) \geq c>0$.

We conclude

$$
d(x)=\lim _{l \rightarrow \infty} d\left(X_{l}, x\right)+s_{l} \geq \lim _{l \rightarrow \infty} d\left(X_{l}, x\right) \geq c>0 .
$$

Now we prove the second equality. At first, let $y \in \pi(\mathcal{Z})$. There exists some $x_{0} \in \mathcal{Z}$ with $\pi\left(x_{0}\right)=y$ and with the recently shown identity we obtain $d\left(x_{0}\right)=0$. Now we get $0 \leq D(y)=\inf _{x \in \pi^{-1}(y)} d(x) \leq d\left(x_{0}\right)=0$.

Now let $y \in P_{0}$ with $0=D(y)=\inf _{x \in \pi^{-1}(y)} d(x)$. Hence there exists some sequence $x_{i} \in \pi^{-1}(y)$ with $\lim _{i \rightarrow \infty} d\left(x_{i}\right)=0$. According to Lemma 6.10 , we get

$$
\pi^{-1}(y) \cap\left\{x \in \mathbb{R}^{N} \mid d(x)<1\right\} \subset \pi^{-1}(y) \cap B(0,6)
$$

and $\pi^{-1}(y) \cap B(0,6)$ is compact. So there exists some accumulation point $a \in \pi^{-1}(y) \cap B(0,6)$ of $\left(x_{i}\right)$ and some subsequence $\left(x_{i_{j}}\right)$ with $\lim _{j \rightarrow \infty} x_{i_{j}}=a$. Since $d$ is continuous, we have $d(a)=0$, which is equivalent to $a \in \mathcal{Z}$. Thus $y \in \pi(\mathcal{Z})$.

According to Lemma $6.9, d$ and $D$ are continuous and hence these sets are closed.

Lemma 6.12. Let $0<\alpha \leq \frac{1}{4}$. There exists some $\bar{\varepsilon}=\bar{\varepsilon}\left(N, n, C_{0}\right)$ so that if $\eta<2 \bar{\varepsilon}$ and $k \geq 4$ for all $\varepsilon \in\left[\frac{\eta}{2}, \bar{\varepsilon}\right)$, there holds:

For all $x, y \in F$ we have

$$
\begin{aligned}
d(x, y) & \leq 6(d(x)+d(y))+2 d(\pi(x), \pi(y)), \\
d\left(\pi^{\perp}(x), \pi^{\perp}(y)\right) & \leq 6(d(x)+d(y))+2 \alpha d(\pi(x), \pi(y)) .
\end{aligned}
$$

Proof. Let $0<\alpha<\frac{1}{4}$ and $k \geq 4$. During this proof, there occur several smallness conditions on $\varepsilon$. The minimum of those will give us the constant $\bar{\varepsilon}$. Let $\eta \leq 2 \varepsilon<2 \bar{\varepsilon}$.

At first, we assume that $d(x)+d(y) \geq 1$. This implies for $x, y \in F \subset B(0,5)$ that

$$
d\left(\pi^{\perp}(x), \pi^{\perp}(y)\right) \leq d(x, y) \leq 10 \leq 10(d(x)+d(y))+2 \alpha d(\pi(x), \pi(y)) .
$$

So for the rest of this proof, we assume that $d(x)+d(y)<1$.

We choose some arbitrary $r_{x} \in(d(x), d(x)+1) \subset(0,2)$. There exists some $(X, t) \in S$ with $d(x) \leq d(X, x)+t<r_{x}$. According to Lemma $6.3(\mathrm{v})$ and because of $t<r_{x}<50$, it follows that $\left(X, r_{x}\right) \in S$. Analogously, for all $r_{y} \in(d(y), d(y)+1)$, we can choose some $Y \in F$ with $d(Y, y)<r_{y}$ and $\left(Y, r_{y}\right) \in S$.

1. Case: $d(X, Y) \leq 2\left(r_{x}+r_{y}\right)$.

We deduce

$$
d\left(\pi^{\perp}(x), \pi^{\perp}(y)\right) \leq d(x, y) \leq d(x, X)+d(X, Y)+d(Y, y) \leq 3 r_{x}+3 r_{y} .
$$

2. Case: $d(X, Y)>2\left(r_{x}+r_{y}\right)$.

We define $B_{1}:=B\left(X, \frac{1}{2} d(X, Y)\right)$ and $B_{2}:=B\left(Y, \frac{1}{2} d(X, Y)\right)$. Using $x, y \in F \subset B(0,5)$, we have

$$
d(X, Y) \leq d(X, x)+d(x, y)+d(y, Y) \leq r_{x}+10+r_{y} \leq d(x)+1+10+d(y)+1<13
$$


With Lemma 6.3 (iv) and $r_{x} \leq \frac{1}{2} d(X, Y)<50, r_{y} \leq \frac{1}{2} d(X, Y)<50$, we obtain $\left(X, \frac{1}{2} d(X, Y)\right)$, $\left(Y, \frac{1}{2} d(X, Y)\right) \in S$. Let $P_{1}$ and $P_{2}$ be the associated planes to $B_{1}$ and $B_{2}$ (see Lemma 6.3 (vi)). Since $S \subset S_{\text {total }}$, we get by definition of $S_{\text {total }}$ and $k \geq 4$ that the requirements of Lemma 5.9 are fulfilled $\left(x=X, y=Y, c=1, \xi=2, t_{x}=t_{y}=\frac{1}{2} d(X, Y), \lambda=\frac{1}{2} \delta\right)$. Hence there exist some constants $C_{3}=C_{3}\left(N, n, C_{0}\right)>1$ and $\varepsilon_{0}=\varepsilon_{0}\left(N, n, C_{0}\right)>0$ so that if $\varepsilon<\varepsilon_{0}$ for $w \in P_{1}$, we obtain

$$
d\left(w, P_{2}\right) \leq C_{3}\left(N, n, C_{0}, \delta\right) \varepsilon\left(\frac{1}{2} d(X, Y)+d(w, X)\right) .
$$

Let $B_{1}^{\prime}:=B\left(X, \frac{1}{2} \varepsilon^{\frac{1}{2 n}} d(X, Y)+r_{x}\right)$ and $B_{2}^{\prime}:=B\left(Y, \frac{1}{2} \varepsilon^{\frac{1}{2 n}} d(X, Y)+r_{y}\right)$. We have $\left(X, \frac{1}{2} \varepsilon^{\frac{1}{2 n}} d(X, Y)+r_{x}\right),\left(Y, \frac{1}{2} \varepsilon^{\frac{1}{2 n}} d(X, Y)+r_{y}\right) \in S$ because $\left(X, r_{x}\right) \in S,\left(Y, r_{y}\right) \in S$ and if $\varepsilon<1$, we have $\frac{1}{2} \varepsilon^{\frac{1}{2 n}} d(X, Y)+r_{x}<7+2<50$. Now we conclude using $S \subset S_{\text {total }}$

$$
\frac{1}{\mu\left(B_{1}^{\prime}\right)} \leq \frac{2}{\delta} \frac{1}{\left(\frac{1}{2} \varepsilon^{\frac{1}{2 n}} d(X, Y)+r_{x}\right)^{n}} \leq \frac{2}{\delta \varepsilon^{\frac{1}{2}}\left(\frac{1}{2} d(X, Y)\right)^{n}} .
$$

Since $k \geq 4, r_{x}<\frac{1}{2} d(X, Y)$ and $\varepsilon<1$, we have

$$
B_{1}^{\prime}=B\left(X, \frac{1}{2} \varepsilon^{\frac{1}{2 n}} d(X, Y)+r_{x}\right) \subset B\left(X, k \frac{1}{2} d(X, Y)\right),
$$

and because $\left(X, \frac{1}{2} d(X, Y)\right) \in S \subset S_{\text {total }}$, we obtain

$$
\beta_{1 ; k}^{P_{1}}\left(X, \frac{1}{2} d(X, Y)\right) \leq 2 \varepsilon .
$$

It follows

$$
\begin{aligned}
\frac{1}{\mu\left(B_{1}^{\prime}\right)} \int_{B_{1}^{\prime}} \frac{d\left(X^{\prime}, P_{1}\right)}{d(X, Y)} \mathrm{d} \mu\left(X^{\prime}\right) \stackrel{(6.4)}{\leq} \frac{2}{\delta \varepsilon^{\frac{1}{2}}\left(\frac{1}{2} d(X, Y)\right)^{n}} \int_{B_{1}^{\prime}} \frac{d\left(X^{\prime}, P_{1}\right)}{d(X, Y)} \mathrm{d} \mu\left(X^{\prime}\right) \\
\stackrel{(6.5)}{\leq} \frac{1}{\delta \varepsilon^{\frac{1}{2}}} \frac{1}{\left(\frac{1}{2} d(X, Y)\right)^{n}} \int_{B\left(X, k \frac{1}{2} d(X, Y)\right)} \frac{d\left(X^{\prime}, P_{1}\right)}{\frac{1}{2} d(X, Y)} \mathrm{d} \mu\left(X^{\prime}\right) \\
=\frac{1}{\delta \varepsilon^{\frac{1}{2}}} \beta_{1 ; k}^{P_{1}}\left(X, \frac{1}{2} d(X, Y)\right) \stackrel{(6.6)}{\leq} \frac{2}{\delta} \varepsilon^{\frac{1}{2}}
\end{aligned}
$$

and analogously

$$
\frac{1}{\mu\left(B_{2}^{\prime}\right)} \int_{B_{2}^{\prime}} \frac{d\left(Y^{\prime}, P_{2}\right)}{d(X, Y)} \mathrm{d} \mu\left(Y^{\prime}\right) \leq \frac{2}{\delta} \varepsilon^{\frac{1}{2}}
$$

With Chebyshev's inequality, we deduce that there exists some $X^{\prime} \in B_{1}^{\prime}$ and some $Y^{\prime} \in B_{2}^{\prime}$ so that $d\left(X^{\prime}, P_{1}\right) \leq \frac{2}{\delta} \varepsilon^{\frac{1}{2}} d(X, Y)$ and $d\left(Y^{\prime}, P_{2}\right) \leq \frac{2}{\delta} \varepsilon^{\frac{1}{2}} d(X, Y)$.

Now let $X_{1}^{\prime}:=\pi_{P_{1}}\left(X^{\prime}\right)$ be the orthogonal projection of $X^{\prime}$ on $P_{1}, Y_{2}^{\prime}:=\pi_{P_{2}}\left(Y^{\prime}\right)$ the orthogonal projection of $Y^{\prime}$ on $P_{2}$, and $X_{12}^{\prime}:=\pi_{P_{2}}\left(X_{1}^{\prime}\right)$ the orthogonal projection of $X_{1}^{\prime}$ on 
$P_{2}$. If $\varepsilon$ is small enough, we have with $\varrho \in\left\{\pi, \pi^{\perp}\right\}$

$$
\begin{aligned}
& d\left(\varrho(X), \varrho\left(X^{\prime}\right)\right) \leq d\left(X, X^{\prime}\right) \leq \frac{1}{2} \varepsilon^{\frac{1}{2 n}} d(X, Y)+r_{x} \\
& d\left(\varrho(Y), \varrho\left(Y^{\prime}\right)\right) \leq d\left(Y, Y^{\prime}\right) \leq \frac{1}{2} \varepsilon^{\frac{1}{2 n}} d(X, Y)+r_{y} \\
& d\left(\varrho\left(X^{\prime}\right), \varrho\left(X_{1}^{\prime}\right)\right) \leq d\left(X^{\prime}, X_{1}^{\prime}\right)=d\left(X^{\prime}, P_{1}\right) \leq \frac{2}{\delta} \varepsilon^{\frac{1}{2}} d(X, Y) \\
& d\left(\varrho\left(Y^{\prime}\right), \varrho\left(Y_{2}^{\prime}\right)\right) \leq d\left(Y^{\prime}, Y_{2}^{\prime}\right)=d\left(Y^{\prime}, P_{2}\right) \leq \frac{2}{\delta} \varepsilon^{\frac{1}{2}} d(X, Y), \\
& d\left(\varrho\left(X_{1}^{\prime}\right), \varrho\left(X_{12}^{\prime}\right)\right) \leq d\left(X_{1}^{\prime}, X_{12}^{\prime}\right)=d\left(X_{1}^{\prime}, P_{2}\right) \\
& \stackrel{6.3)}{\leq} C_{3} \varepsilon\left(\frac{1}{2} d(X, Y)+d\left(X_{1}^{\prime}, X\right)\right) \\
& \leq C_{3} \varepsilon\left(\frac{1}{2} d(X, Y)+d\left(X_{1}^{\prime}, X^{\prime}\right)+d\left(X^{\prime}, X\right)\right) \\
& \leq C_{3} \varepsilon\left(\frac{1}{2} d(X, Y)+\frac{2}{\delta} \varepsilon^{\frac{1}{2}} d(X, Y)+\frac{1}{2} \varepsilon^{\frac{1}{2 n}} d(X, Y)+r_{x}\right) \\
&<2 C_{3} \varepsilon d(X, Y) .
\end{aligned}
$$

According to Lemma 6.3 (vi), we have $\Varangle\left(P_{2}, P_{0}\right) \leq \alpha$ and we get with Corollary $2.24\left(X_{12}^{\prime}, Y_{2}^{\prime} \in\right.$ $\left.P_{2}\right)$ using $\alpha \leq \frac{1}{4}$

$$
\begin{aligned}
d\left(X_{12}^{\prime}, Y_{2}^{\prime}\right) & \leq \frac{1}{1-\alpha} d\left(\pi\left(X_{12}^{\prime}\right), \pi\left(Y_{2}^{\prime}\right)\right) \leq 2 d\left(\pi\left(X_{12}^{\prime}\right), \pi\left(Y_{2}^{\prime}\right)\right), \\
d\left(\pi^{\perp}\left(X_{12}^{\prime}\right), \pi^{\perp}\left(Y_{2}^{\prime}\right)\right) & \leq \frac{\alpha}{1-\alpha} d\left(\pi\left(X_{12}^{\prime}\right), \pi\left(Y_{2}^{\prime}\right)\right) \leq \frac{4}{3} \alpha d\left(\pi\left(X_{12}^{\prime}\right), \pi\left(Y_{2}^{\prime}\right)\right) .
\end{aligned}
$$

Inserting the intermediate points $X^{\prime}, X_{1}^{\prime}, X_{12}^{\prime}, Y_{2}^{\prime}, Y^{\prime}$ using triangle inequality twice and using inequalities (6.7), (6.8) and (6.9), there exists some constant $C$ so that

$$
\begin{aligned}
d(X, Y) & \leq C \frac{1}{\delta} \varepsilon^{\frac{1}{2 n}} d(X, Y)+r_{x}+r_{y}+2 d\left(\pi\left(X_{12}^{\prime}\right), \pi\left(Y_{2}^{\prime}\right)\right) \\
& \leq C \frac{1}{\delta} \varepsilon^{\frac{1}{2 n}} d(X, Y)+3\left(r_{x}+r_{y}\right)+2 d(\pi(X), \pi(Y))
\end{aligned}
$$

and hence if $\varepsilon$ fulfils $C \frac{1}{\delta} \varepsilon^{\frac{1}{2 n}} \leq \frac{1}{2}$, we get

$$
d(X, Y) \leq 6\left(r_{x}+r_{y}\right)+4 d(\pi(X), \pi(Y)) .
$$

As for $d(X, Y)$, we estimate $d\left(\pi^{\perp}(X), \pi^{\perp}(Y)\right)$ by repeated use of the triangle inequality and (6.10). With (6.11), we deduce

$$
\begin{aligned}
& d\left(\pi^{\perp}(X), \pi^{\perp}(Y)\right) \\
\leq & C \frac{1}{\delta} \varepsilon^{\frac{1}{2 n}} d(X, Y)+3\left(r_{x}+r_{y}\right)+\frac{4}{3} \alpha d(\pi(X), \pi(Y)) \\
\stackrel{(6.11)}{\leq} & C \frac{1}{\delta} \varepsilon^{\frac{1}{2 n}}\left[6\left(r_{x}+r_{y}\right)+4 d(\pi(X), \pi(Y))\right]+3\left(r_{x}+r_{y}\right)+\frac{4}{3} \alpha d(\pi(X), \pi(Y)) \\
\leq & 4\left(r_{x}+r_{y}\right)+2 \alpha d(\pi(X), \pi(Y)) .
\end{aligned}
$$


We get using $d\left(\pi^{\perp}(x), \pi^{\perp}(X)\right) \leq d(x, X) \leq r_{x}$ and $d\left(\pi^{\perp}(Y), \pi^{\perp}(y)\right) \leq d(Y, y) \leq r_{y}$

$$
\begin{aligned}
d\left(\pi^{\perp}(x), \pi^{\perp}(y)\right) & \leq d(x, X)+d\left(\pi^{\perp}(X), \pi^{\perp}(Y)\right)+d(Y, y) \\
& \leq 5\left(r_{x}+r_{y}\right)+2 \alpha d(\pi(X), \pi(Y)) \\
& \leq 5\left(r_{x}+r_{y}\right)+2 \alpha[d(X, x)+d(\pi(x), \pi(y))+d(y, Y)] \\
& \leq 6\left(r_{x}+r_{y}\right)+2 \alpha d(\pi(x), \pi(y)) .
\end{aligned}
$$

Hence, in both cases, we obtain

$$
d\left(\pi^{\perp}(x), \pi^{\perp}(y)\right) \leq 6\left(r_{x}+r_{y}\right)+2 \alpha d(\pi(x), \pi(y)) .
$$

Since $r_{x}$ and $r_{y}$ were arbitrarily chosen with $d(x)<r_{x}$ and $d(y)<r_{y}$, we finally get

$$
d\left(\pi^{\perp}(x), \pi^{\perp}(y)\right) \leq 6(d(x)+d(y))+2 \alpha d(\pi(x), \pi(y)) .
$$

From this, we deduce

$$
d(x, y) \leq d(\pi(x), \pi(y))+d\left(\pi^{\perp}(x), \pi^{\perp}(y)\right) \leq 6(d(x)+d(y))+(1+2 \alpha) d(\pi(x), \pi(y)) .
$$

\subsection{A Whitney-type decomposition of $P_{0} \backslash \pi(\mathcal{Z})$}

In this part, we show that $P_{0} \backslash \pi(\mathcal{Z})$ can be decomposed as a union of disjoint cubes $R_{i}$, where the diameter of $R_{i}$ is proportional to $D(x)$ for all $x \in R_{i}$. This result is a variant of the Whitney decomposition for open sets in $\mathbb{R}^{n}$, cf. [Gra08, Appendix J].

Definition 6.13 (Dyadic primitive cells).

1. We define the set of dyadic primitive cells in $\mathbb{R}^{n}$

$$
\tilde{\mathcal{D}}:=\left\{Q_{l,\left(d_{1}, \ldots, d_{n}\right)} \mid d_{i} \in \mathbb{Z}, i \in\{1 \ldots, n\}, l \in \mathbb{N}\right\}
$$

where

$$
Q_{l,\left(d_{1}, \ldots, d_{n}\right)}:=\left\{\left(x_{1}, \ldots, x_{n}\right) \in \mathbb{R}^{n} \mid \frac{d_{i}}{2^{l}} \leq x_{i}<\frac{d_{i}+1}{2^{l}}, i \in\{1 \ldots, n\}\right\}
$$

2. The plane $P_{0}$ is an $n$-dimensional linear subspace of $\mathbb{R}^{N}$ and therefore, isomorph to $\mathbb{R}^{n}$. Now we set $\mathcal{D}$ to be the set of all dyadic primitive cells on $P_{0}$ in the same manner as $\tilde{\mathcal{D}}$ on $\mathbb{R}^{n}$.

3. Let $r \in(0, \infty)$ and $Q$ be some cube in $\mathbb{R}^{N}$. By $r Q$, we denote the cube with the same centre and orientation as $Q$ but $r$-times the diameter.

We mention that the function $D$ depends on the choice of $\alpha$ and $\varepsilon$ because $D$ depends on the set $S \subset S_{\text {total }}^{\varepsilon, \alpha}$. Hence the family of cubes given by the following lemma depends on the choice of $\alpha$ and $\varepsilon$ as well. 
Lemma 6.14. Let $\alpha, \varepsilon>0$. If $\eta \leq 2 \varepsilon$, then there exists a countable family of cubes $\left\{R_{i}\right\}_{i \in I} \subset$ $\mathcal{D}$ such that

(i) $10 \operatorname{diam} R_{i} \leq D(x) \leq 50$ diam $R_{i}$ for all $x \in 10 R_{i}$,

(ii) $P_{0} \backslash \pi(\mathcal{Z})=\bigcup_{i \in I} R_{i}=\bigcup_{i \in I} 2 R_{i}$ and cubes $R_{i}$ have disjoint interior,

(iii) for every $i, j \in I$ with $10 R_{i} \cap 10 R_{j} \neq \emptyset$, we have $\frac{1}{5} \operatorname{diam} R_{j} \leq \operatorname{diam} R_{i} \leq 5 \operatorname{diam} R_{j}$,

(iv) for every $i \in I$, there are at most $180^{n}$ cells $R_{j}$ with $10 R_{i} \cap 10 R_{j} \neq \emptyset$.

Proof. For $z \in P_{0}, D(z)>0$, we define $Q_{z} \in \mathcal{D}$ as the largest dyadic primitive cell that contains $z$ and fulfils

$$
\operatorname{diam} Q_{z} \leq \frac{1}{20} \inf _{u \in Q_{z}} D(u)
$$

At first, we show that for such a given $z$ the cell $Q_{z}$ exists and is unique. The function $D$ is continuous and $D(z)>0$. Hence if we choose a small enough dyadic primitive cell $Q$ that contains $z$, we get $\operatorname{diam} Q \leq \frac{1}{20} \inf _{u \in Q} D(u)$. Due to the dyadic structure, there can only be one largest dyadic primitive cell that contains $z$ and fulfils (6.12).

We choose the smallest countable family of cubes $\left\{R_{i}\right\}_{i \in I}$ where $R_{i} \in \mathcal{D}$ such that $\left\{R_{i} \mid i \in\right.$ $I\}=\left\{Q_{z} \in \mathcal{D} \mid z \in P_{0}, D(z)>0\right\}$. This implies that $R_{i}=R_{j}$ is equivalent to $i=j$.

(i) Let $x \in 10 R_{i}$ and $u \in R_{i}$. We get

$$
20 \operatorname{diam} R_{i} \leq \inf _{v \in R_{i}} D(v) \leq D(u) \leq D(x)+d(x, u)<D(x)+10 \operatorname{diam} R_{i},
$$

and hence $10 \operatorname{diam} R_{i} \leq D(x)$.

Let $J_{i} \in \mathcal{D}$ be the smallest cell in $\mathcal{D}$ with $R_{i} \subsetneq J_{i}$ and choose $u \in J_{i}$ so that $D(u)<$ $20 \operatorname{diam} J_{i}=40 \operatorname{diam} R_{i}$. This is possible because otherwise $R_{i}$ is not maximal relating to $\operatorname{diam} R_{i} \leq \frac{1}{20} \inf _{v \in R_{i}} D(v)$. We obtain

$$
D(x) \leq D(u)+d(u, x)<D(u)+10 \operatorname{diam} R_{i} \leq 50 \operatorname{diam} R_{i} .
$$

(ii) If the interior of some cells $R_{i}$ and $R_{j}$ were not disjoint, because of the dyadic structure, one cell would be contained in the other. But then one of those would not be the maximal cell fulfilling (6.12). Hence the $R_{i}$ 's have disjoint interior.

Now we prove $P_{0} \backslash \pi(\mathcal{Z})=\bigcup_{i \in I} R_{i}=\bigcup_{i \in I} 2 R_{i}$. For all $x \in 2 R_{i}$, we have with (i) $0<$ $10 \operatorname{diam} R_{i} \leq D(x)$ which implies with Lemma $6.11 x \notin \pi(\mathcal{Z})$. Hence we get $P_{0} \backslash \pi(\mathcal{Z}) \supset$ $\bigcup_{i \in I} 2 R_{i}$. Now let $x \in P_{0} \backslash \pi(\mathcal{Z})$. With Lemma 6.11 , we get $D(x)>0$. So there exists the cube $Q_{x} \in \mathcal{D}$ with $x \in Q_{x}$ and hence $x \in \bigcup_{i \in I} R_{i}$.

(iii) Let $10 R_{i} \cap 10 R_{j} \neq \emptyset$. Then there exists some $x \in 10 R_{i} \cap 10 R_{j}$ and, with (i), we get

$$
\frac{10}{50} \operatorname{diam} R_{j} \leq \frac{1}{50} D(x) \leq \operatorname{diam} R_{i} \leq \frac{1}{10} D(x) \leq \frac{50}{10} \operatorname{diam} R_{j} .
$$

(iv) Let $i \in I$ and $R_{j}$ with $10 R_{i} \cap 10 R_{j} \neq \emptyset$. We conclude

$$
d\left(R_{i}, R_{j}\right)=\inf _{x \in R_{i}, y \in R_{j}} d(x, y)<5 \operatorname{diam} R_{i}+5 \operatorname{diam} R_{j} \leq 30 \operatorname{diam} R_{i}
$$


and so $R_{j} \subset(1+30+5) R_{i}$. Furthermore, we have $\operatorname{diam} R_{j} \geq \frac{1}{5} \operatorname{diam} R_{i}$. Since the cells $R_{j}$ are disjoint, there exist at most

$$
\frac{\mathcal{H}^{n}\left(36 R_{i}\right)}{\mathcal{H}^{n}\left(R_{j}\right)} \leq\left(\frac{36 \operatorname{diam} R_{i}}{\frac{1}{5} \operatorname{diam} R_{i}}\right)^{n}=(180)^{n}
$$

cells $R_{j}$ with $10 R_{i} \cap 10 R_{j} \neq \emptyset$.

Now we set $U_{12}:=B(0,12) \cap P_{0}$ and $I_{12}:=\left\{i \in I \mid R_{i} \cap U_{12} \neq \emptyset\right\}$.

Lemma 6.15. Let $\alpha, \varepsilon>0$. If $\eta \leq 2 \varepsilon$, we have that for every $i \in I_{12}$, there exists some ball $B_{i}=B\left(X_{i}, t_{i}\right)$ with $\left(X_{i}, t_{i}\right) \in S$ and

(i) $\operatorname{diam} R_{i} \leq \operatorname{diam} B_{i} \leq 200 \operatorname{diam} R_{i}$,

(ii) $d\left(\pi\left(B_{i}\right), R_{i}\right) \leq 100 \operatorname{diam} R_{i}$.

Proof. Let $i \in I_{12}$. For $x \in R_{i}$, there exists some $(X, t) \in S$ so that we get with Lemma 6.8, Lemma 6.11 and Lemma 6.14 (i), (ii)

$$
d(\pi(X), x)+t \leq 2 D(x) \leq 100 \operatorname{diam} R_{i} .
$$

Choose $B_{i}:=B\left(X_{i}, t_{i}\right):=B(X, r)$ with $r=\max \left\{t, \frac{\operatorname{diam} R_{i}}{2}\right\}$. For any $z \in F \subset B(0,5)$, we have $(z, 40) \in S$ (Lemma 6.3 (iv)) and there exists some $y \in R_{i} \cap B(0,12)$ which implies using Lemma 6.14 (i) that $10 \operatorname{diam} R_{i} \leq D(y) \leq d(\pi(z), y)+40 \leq 5+12+40=57$. Hence in both cases $t \leq r<50$. With Lemma $6.3(\mathrm{v})$, we have $(X, r) \in S$ and we obtain

$$
\operatorname{diam} R_{i} \leq 2 r=\operatorname{diam} B_{i}=\left\{\begin{array}{c}
2 t \stackrel{(6.13)}{\leq} 200 \operatorname{diam} R_{i} \\
\operatorname{diam} R_{i}
\end{array}\right\} \leq 200 \operatorname{diam} R_{i}
$$

and

$$
d\left(\pi\left(B_{i}\right), R_{i}\right) \leq d(\pi(X), x) \stackrel{(6.13)}{\leq} 100 \operatorname{diam} R_{i}
$$

\subsection{Construction of the function $A$}

We recall that $\pi:=\pi_{P_{0}}: \mathbb{R}^{N} \rightarrow P_{0}$ is the orthogonal projection on $P_{0}$ and introduce $\pi^{\perp}:=$ $\pi_{P_{0}}^{\perp}: \mathbb{R}^{N} \rightarrow P_{0}^{\perp}$, the orthogonal projection on $P_{0}^{\perp}$, where $P_{0}^{\perp}:=\left\{x \in \mathbb{R}^{N} \mid x \cdot v=0\right.$ for all $v \in$ $\left.P_{0}\right\}$ is the orthogonal complement of $P_{0}$. To define the function $A$, we want to invert the projection $\left.\pi\right|_{\mathcal{Z}}$ on $\mathcal{Z}$.

Lemma 6.16. Let $0<\alpha \leq \frac{1}{4}$. There exists some $\bar{\varepsilon}=\bar{\varepsilon}\left(N, n, C_{0}\right)$ so that if $\eta<2 \bar{\varepsilon}$ and $k \geq 4$ for all $\varepsilon \in\left[\frac{\eta}{2}, \bar{\varepsilon}\right)$, the orthogonal projection $\left.\pi\right|_{\mathcal{Z}}: \mathcal{Z} \rightarrow P_{0}$ is injective.

Proof. Let $x, y \in \mathcal{Z}$ with $\pi(x)=\pi(y)$, which is equivalent to $d(\pi(x), \pi(y))=0$. With Lemma 6.11 , we obtain $d(x)=0=d(y)$ and so, with Lemma 6.12 , we conclude $d(x, y)=0$. So we get $x=y$ and hence $\pi$ is injective on $\mathcal{Z}$. 
Since $\left.\pi\right|_{\mathcal{Z}}: \mathcal{Z} \rightarrow P_{0}$ is injective, we are able to define the desired Lipschitz function $A$ on $\pi(\mathcal{Z})$ by

$$
A(a):=\pi^{\perp}\left(\left.\pi\right|_{\mathcal{Z}} ^{-1}(a)\right)
$$

where $a \in \pi(\mathcal{Z})$.

Lemma 6.17. Under the conditions of the previous lemma, the map $A: \pi(\mathcal{Z}) \rightarrow P_{0}^{\perp}$ is $2 \alpha$-Lipschitz.

Proof. The projection $\pi$ is injective on $\mathcal{Z}$ (Lemma 6.16), so for $a, b \in \pi(\mathcal{Z})$ with $a \neq b$, there exist distinct $X, Y \in \mathcal{Z}$ with $\pi(X)=a$ and $\pi(Y)=b$. We have $A(a)=A(\pi(X))=\pi^{\perp}(X)$, $A(b)=\pi^{\perp}(Y)$ and, since $X, Y \in \mathcal{Z}$, we conlude with Lemma 6.11 that $d(X)=d(Y)=0$. So, with Lemma 6.12 , we get

$$
d(A(a), A(b))=d\left(\pi^{\perp}(X), \pi^{\perp}(Y)\right) \leq 0+2 \alpha d(\pi(X), \pi(Y))=2 \alpha d(a, b) .
$$

Now we have a Lipschitz function $A$ defined on $\pi(\mathcal{Z})$. By using Kirszbraun's theorem [Fed69, Thm 2.10.43], we would obtain a Lipschitz extension of $A$ defined on $P_{0}$ with the same Lipschitz constant $2 \alpha$, where the graph of the extension covers $\mathcal{Z}$. But until now, we do not know that $\mathcal{Z}$ is a major part of $F$. We cannot even be sure that $\mathcal{Z}$ is not a null set. So we do not use Kirszbraun's theorem here, but we will extend $A$ by an explicit construction. This will help us to show that the other parts of $F$, in particular $F_{1}, F_{2}, F_{3}$, are quite small.

Definition 6.18. Let $\alpha, \varepsilon>0$. If $\eta \leq 2 \varepsilon$, for all $i \in I_{12}$, we set $P_{i}:=P_{\left(X_{i}, t_{i}\right)}$, where $P_{\left(X_{i}, t_{i}\right)}$ is the $n$-dimensional plane, which is, in the sense of Lemma $6.3(\mathrm{vi})$, associated to the ball $B\left(X_{i}, t_{i}\right)=B_{i}$ given by Lemma 6.15 .

Lemma 6.19. Let $0<\alpha \leq \frac{1}{2}$ and $\varepsilon>0$. If $\eta \leq 2 \varepsilon$, then for all $i \in I_{12}$, there exists some affine map $A_{i}: P_{0} \rightarrow P_{0}^{\perp}$ with graph $G\left(A_{i}\right)=P_{i}$ and $A_{i}$ is $2 \alpha$-Lipschitz.

Proof. We know $\Varangle\left(P_{i}, P_{0}\right) \leq \alpha \leq \frac{1}{2}$ (cf. definition of $S_{\text {total }}$ ) and, with Corollary 2.25, there exists some affine map $A_{i}: P_{0} \rightarrow P_{0}^{\perp}$ with graph $G\left(A_{i}\right)=P_{i}$. Using Corollary 2.24, we get for all $x, y \in P_{0}$

$$
\begin{aligned}
\left|A_{i}(x)-A_{i}(y)\right| & =d\left(\pi^{\perp}\left(A_{i}(x)+x\right), \pi^{\perp}\left(A_{i}(y)+y\right)\right) \\
& \leq \frac{\alpha}{1-\alpha} d\left(\pi\left(A_{i}(x)+x\right), \pi\left(A_{i}(y)+y\right)\right) \leq 2 \alpha|x-y| .
\end{aligned}
$$

In the following, we use differentiable functions defined on subsets of $P_{0}$. For the definition of the derivative see section A.2 on page 156.

Lemma 6.20. Let $\alpha, \varepsilon>0$. If $\eta \leq 2 \varepsilon$, then there exists some partition of unity $\phi_{i} \in C^{\infty}\left(U_{12}, \mathbb{R}\right), i \in I_{12}$, and some constant $C=C(n)$ with

- $0 \leq \phi_{i} \leq 1$ on $U_{12}$ and $\phi_{i} \equiv 0$ on the exterior of $3 R_{i}$, 
- $\sum_{i \in I_{0}} \phi_{i}(a)=1$ for all $a \in U_{12}$,

- $\left|\partial^{\omega} \phi_{i}(a)\right| \leq \frac{C(n)}{\left(\operatorname{diam} R_{i}\right)^{|\omega|}}$ where $\omega$ is some multi-index with $1 \leq|\omega| \leq 2$.

Proof. For every $i \in I_{12}$, we choose some function $\tilde{\phi}_{i} \in \mathcal{C}^{\infty}\left(P_{0}, \mathbb{R}\right)$ with $0 \leq \tilde{\phi}_{i} \leq 1, \tilde{\phi}_{i} \equiv 1$ on $2 R_{i}, \tilde{\phi}_{i} \equiv 0$ on the exterior of $3 R_{i},\left|\partial^{\omega} \tilde{\phi}_{i}\right| \leq \frac{C}{\operatorname{diam} R_{i}}$ for all multi-indices $\omega$ with $|\omega|=1$ and $\left|\partial^{\kappa} \tilde{\phi}_{i}\right| \leq \frac{C}{\left(\operatorname{diam} R_{i}\right)^{2}}$ for all multi-indices $\kappa$ with $|\kappa|=2$. Now on $V:=\bigcup_{i \in I_{12}} 2 R_{i}$, we can define the partition of unity

$$
\phi_{i}(a):=\frac{\tilde{\phi}_{i}(a)}{\sum_{j \in I_{12}} \tilde{\phi}_{j}(a)} .
$$

For all $a \in V$, there exists some $i \in I_{12}$ with $a \in 2 R_{i}$ and hence $\sum_{j \in I_{12}} \tilde{\phi}_{j}(a) \geq 1$. Moreover, due to Lemma 6.14 (iv), there are only finitely many $j \in I_{12}$ such that $\tilde{\phi}_{j}(a) \neq 0$. Hence $\phi_{i}$ is well defined and fulfils the first and the second condition. With Lemma 6.14 (iv), $a \in V$ and $|\omega|=1$, we obtain

$$
\begin{aligned}
\left|\partial^{\omega} \phi_{i}(a)\right| & \leq \frac{\left|\left(\partial^{\omega} \tilde{\phi}_{i}(a)\right) \sum_{j \in I_{12}} \tilde{\phi}_{j}(a)-\tilde{\phi}_{i}(a) \sum_{j \in I_{12}} \partial^{\omega} \tilde{\phi}_{j}(a)\right|}{\left|\sum_{j \in I_{12}} \tilde{\phi}_{j}(a)\right|^{2}} \\
& \leq\left|\partial^{\omega} \tilde{\phi}_{i}(a)\right|+\left|\tilde{\phi}_{i}(a) \sum_{j \in I_{12}} \partial^{\omega} \tilde{\phi}_{j}(a)\right| \\
& \leq \frac{C}{\operatorname{diam} R_{i}}+\left|\sum_{j \in I_{12}} \partial^{\omega} \tilde{\phi}_{j}(a)\right| \\
& \leq \frac{C}{\operatorname{diam} R_{i}}+(180)^{n} \frac{C}{\operatorname{diam} R_{i}} \\
& \leq \frac{C(n)}{\operatorname{diam} R_{i}},
\end{aligned}
$$

and accordingly for $|\omega|=2$

$$
\left|\partial^{\omega} \phi_{i}(a)\right| \leq \frac{C(n)}{\left(\operatorname{diam} R_{i}\right)^{2}}
$$

Definition 6.21 (Definition of $A$ on $U_{12}$ ). Let $\alpha, \varepsilon>0$. If $\eta \leq 2 \varepsilon$ and $k \geq 4$, we extend the function $A: \pi(\mathcal{Z}) \rightarrow P_{0}^{\perp} \subset \mathbb{R}^{N}, a \mapsto \pi^{\perp}\left(\left.\pi\right|_{\mathcal{Z}} ^{-1}(a)\right)$ (see page 77 ) to the whole set $U_{12}$ by setting for $a \in U_{12}$

$$
A(a):= \begin{cases}\pi^{\perp}\left(\left.\pi\right|_{\mathcal{Z}} ^{-1}(a)\right) & , a \in \pi(\mathcal{Z}) \\ \sum_{i \in I_{12}} \phi_{i}(a) A_{i}(a) & , a \in U_{12} \cap \bigcup_{i \in I_{12}} 2 R_{i} .\end{cases}
$$

With $\mathcal{Z} \subset F \subset B(0,5)$, we get $\pi(\mathcal{Z}) \subset U_{12}$ and, with Lemma 6.14 (ii), we obtain $\bigcup_{i \in I_{12}} 2 R_{i} \cap \pi(\mathcal{Z})=\emptyset$, hence we have defined $A$ on the whole set $U_{12}=\left(U_{12} \cap \bigcup_{i \in I_{12}} 2 R_{i}\right) \dot{U} \pi(\mathcal{Z})$. 


\section{5 $A$ is Lipschitz continuous}

In this section, we show that $A$ is Lipschitz continuous. We start with some useful estimates.

Lemma 6.22. Let $0<\alpha \leq \frac{1}{4}$. There exists some $\bar{k} \geq 4$ and some $\bar{\varepsilon}=\bar{\varepsilon}\left(N, n, C_{0}\right)$ so that if $k \geq \bar{k}$ and $\eta<2 \bar{\varepsilon}$ for all $\varepsilon \in\left[\frac{\eta}{2}, \bar{\varepsilon}\right)$, there exist some constants $C>1$ and $\bar{C}=\bar{C}\left(N, n, C_{0}\right)>1$ so that for all $i, j \in I_{12}$ with $i \neq j$ and $10 R_{i} \cap 10 R_{j} \neq \emptyset$, we get

(i) $d\left(B_{i}, B_{j}\right) \leq C \operatorname{diam} R_{j}$,

(ii) $d\left(A_{i}(q), A_{j}(q)\right) \leq \bar{C} \varepsilon \operatorname{diam} R_{j}$ for all $q \in 100 R_{j}$,

(iii) the Lipschitz constant of the map $\left(A_{i}-A_{j}\right): P_{0} \rightarrow P_{0}^{\perp}$ fulfils $\operatorname{Lip}_{A_{i}-A_{j}} \leq \bar{C} \varepsilon$,

(iv) $d\left(A(u), A_{j}(u)\right) \leq \bar{C} \varepsilon \operatorname{diam} R_{j}$ for all $u \in 2 R_{j} \cap U_{12}$.

Proof. Let $0<\alpha \leq \frac{1}{4}$. We set $\bar{\varepsilon}=\min \left\{\frac{\delta}{2}, \bar{\varepsilon}^{\prime}, \varepsilon_{0}\right\}$, where $\delta=\delta(N, n)$ is defined on page $65, \bar{\varepsilon}^{\prime}$ is the upper bound for $\varepsilon$ given by Lemma 6.12 and $\varepsilon_{0}$ is the constant from Lemma 5.9. Let $\eta<2 \bar{\varepsilon}$ and choose $\varepsilon$ such that $\eta \leq 2 \varepsilon<2 \bar{\varepsilon}$.

(i) Let $B_{i}=B\left(X_{i}, t_{i}\right)$ and $B_{j}=B\left(X_{j}, t_{j}\right)$. With Lemma 6.14 and Lemma 6.15 , we get

$$
d\left(\pi\left(X_{i}\right), \pi\left(X_{j}\right)\right) \leq d\left(\pi\left(X_{i}\right), R_{i}\right)+d\left(R_{i}, R_{j}\right)+d\left(\pi\left(X_{j}\right), R_{j}\right)+C \operatorname{diam} R_{i} \leq C \operatorname{diam} R_{j},
$$

and for $l \in\{i, j\}$, we have $\left(X_{l}, t_{l}\right) \in S$ and hence

$$
d\left(X_{l}\right) \leq d\left(X_{l}, X_{l}\right)+t_{l}=\frac{1}{2} \operatorname{diam} B_{l} \leq 100 \operatorname{diam} R_{l} \leq 500 \operatorname{diam} R_{j} .
$$

Now, with Lemma 6.12 , there exists some constant $C>1$ with

$$
d\left(B_{i}, B_{j}\right) \leq d\left(X_{i}, X_{j}\right) \leq C \operatorname{diam} R_{j} .
$$

(ii) At first, we show for $q \in 100 R_{j}$ that $d\left(A_{i}(q)+q, X_{i}\right) \leq C \operatorname{diam} R_{j}$. Since $\left(X_{i}, t_{i}\right) \in$ $S \subset S_{\text {total }}$ and $\varepsilon \leq \frac{\delta}{4}$, the requirements of Lemma 5.10 (ii) are fulfilled for $\sigma=2 \varepsilon, x=X_{i}$, $t=t_{i}, \lambda=\frac{1}{2} \delta, P=P_{i}$ and we get $B\left(X_{i}, 2 t_{i}\right) \cap P_{i} \neq \emptyset$. Thus there exists some $a \in P_{0}$ with $A_{i}(a)+a \in B\left(X_{i}, 2 t_{i}\right) \cap P_{i}$ and $a \in \pi\left(2 B_{i}\right)$. Let $b \in R_{i}$. Since $A_{i}$ is $2 \alpha$-Lipschitz and $\alpha<\frac{1}{2}$, we obtain using Lemma 6.14 and 6.15

$$
\begin{aligned}
d\left(A_{i}(q)+q, X_{i}\right) & \leq d\left(A_{i}(q)+q, A_{i}(a)+a\right)+d\left(A_{i}(a)+a, X_{i}\right) \\
& \leq\left|A_{i}(q)-A_{i}(a)\right|+d(q, a)+\operatorname{diam} B_{i} \\
& \leq 2 d(q, a)+\operatorname{diam} B_{i} \\
& \leq 2[d(q, b)+d(b, a)]+\operatorname{diam} B_{i} \leq C \operatorname{diam} R_{j} .
\end{aligned}
$$

With Lemma 6.14 and 6.15, there exists some constant $C>2$ so that $\frac{1}{C} t_{j} \leq t_{i} \leq C t_{j}$. Moreover, we have $\left(X_{i}, t_{i}\right),\left(X_{j}, t_{j}\right) \in S \subset S_{\text {total }}$ (Lemma 6.15) and hence $\beta_{1 ; k}^{P_{i}}\left(X_{i}, t_{i}\right) \leq 2 \varepsilon$ and $\beta_{1 ; k}^{P_{j}}\left(X_{j}, t_{j}\right) \leq 2 \varepsilon$ as well as $\delta\left(B\left(X_{i}, t_{i}\right)\right) \geq \frac{\delta}{2}$ and $\delta\left(B\left(X_{j}, t_{j}\right)\right) \geq \frac{\delta}{2}$. We have already seen in the proof of (i) that $d\left(X_{i}, X_{j}\right) \leq C t_{j}$. So, with $k \geq \bar{k}:=2 C^{2} \geq 4 C$, the requirements of Lemma 5.9 (i) are fulfilled for $x=X_{j}, y=X_{i}, c=C, \xi=2, t_{x}=t_{j}, t_{y}=t_{i} \lambda=\frac{\delta}{2}$, and hence 
there exists some $\varepsilon_{0}>0$ and some constant $C_{3}=C_{3}\left(N, n, C_{0}\right)>1$ so that, for $\varepsilon<\bar{\varepsilon} \leq \varepsilon_{0}$, we get

$$
d\left(A_{i}(q)+q, P_{j}\right) \leq C_{3} \varepsilon\left(t_{j}+d\left(A_{i}(q)+q, X_{j}\right)\right) .
$$

Furthermore, there exists some $o \in P_{0}$ so that $A_{j}(o)+o=\pi_{P_{j}}\left(A_{i}(q)+q\right)$. Now, with Lemma 6.14, Lemma 6.15 and using that $A_{i}$ is Lipschitz continuous with Lipschitz constant $2 \alpha<1$, we obtain

$$
\begin{aligned}
d\left(A_{i}(q), A_{j}(q)\right) & =d\left(A_{i}(q)+q, A_{j}(q)+q\right) \\
& \leq d\left(A_{i}(q)+q, P_{j}\right)+d\left(A_{j}(o)+o, A_{j}(q)+q\right) \\
& <d\left(A_{i}(q)+q, P_{j}\right)+2 d(o, q) \\
& \leq d\left(A_{i}(q)+q, P_{j}\right)+2 d\left(A_{i}(q)+q, A_{j}(o)+o\right) \\
& \stackrel{(6.15)}{\leq} 3 C_{3} \varepsilon\left(\operatorname{diam} B_{j}+d\left(A_{i}(q)+q, X_{i}\right)+d\left(X_{i}, X_{j}\right)\right) \\
& \stackrel{(6.14)}{\leq} C\left(N, n, C_{0}\right) \varepsilon \operatorname{diam} R_{j} .
\end{aligned}
$$

(iii) Without loss of generality, we assume diam $R_{i} \leq \operatorname{diam} R_{j}$. We have $B\left(y, 2 \operatorname{diam} R_{i}\right) \cap P_{0} \subset$ $20 R_{i} \cap 20 R_{j}$ for some $y \in 10 R_{i} \cap 10 R_{j} \neq \emptyset$. We choose $a, b \in B\left(y, 2 \operatorname{diam} R_{i}\right) \cap P_{0}$ with $d(a, b) \geq \operatorname{diam} R_{i}$. Now, with (ii), we get

$$
\begin{aligned}
\left|\left(A_{i}-A_{j}\right)(a)-\left(A_{i}-A_{j}\right)(b)\right| & \leq\left|A_{i}(a)-A_{j}(a)\right|+\left|A_{i}(b)-A_{j}(b)\right| \\
& \leq C \varepsilon \operatorname{diam} R_{i} \leq C\left(N, n, C_{0}\right) \varepsilon d(a, b) .
\end{aligned}
$$

Since $A_{i}$ and $A_{j}$ are affine maps, the map $A_{i}-A_{j}$ is also affine. The estimates above hold for all $a, b \in B\left(y, 2 \operatorname{diam} R_{i}\right) \cap P_{0}$ with $d(a, b) \geq \operatorname{diam} R_{i}$, which implies that the affine map $A_{i}-A_{j}$ is Lipschitz continuous with Lipschitz constant $C\left(N, n, C_{0}\right) \varepsilon$.

(iv) Let $u \in 2 R_{j} \cap U_{12}$. Using $\sum_{l \in I_{12}} \phi_{l}(u)=1$ and Lemma 6.14 (iv), we obtain

$$
\begin{aligned}
d\left(A(u), A_{j}(u)\right) & =\left|\sum_{l \in I_{12}} \phi_{l}(u) A_{l}(u)-\sum_{l \in I_{12}} \phi_{l}(u) A_{j}(u)\right| \\
& \leq \sum_{l \in I_{12}} \phi_{l}(u)\left|A_{l}(u)-A_{j}(u)\right| \stackrel{(\mathrm{ii})}{\leq} \bar{C}\left(N, n, C_{0}\right) \varepsilon \operatorname{diam} R_{j} .
\end{aligned}
$$

Lemma 6.23. Let $0<\alpha \leq \frac{1}{4}$. There exists some $\bar{k} \geq 4$ and some $\bar{\varepsilon}=\bar{\varepsilon}\left(N, n, C_{0}, \alpha\right)<\alpha$ so that if $k \geq \bar{k}$ and $\eta<2 \bar{\varepsilon}$ for all $\varepsilon \in\left[\frac{\eta}{2}, \bar{\varepsilon}\right)$, the function $A$ is Lipschitz continuous on $2 R_{j} \cap U_{12}$ for all $j \in I_{12}$ with Lipschitz constant $3 \alpha$.

Proof. Let $0<\alpha \leq \frac{1}{4}$. We set $\bar{\varepsilon}:=\min \left\{\bar{\varepsilon}^{\prime}, \frac{\alpha}{\tilde{C}}\right\}$, where $\bar{\varepsilon}^{\prime}$ is the upper bound for $\varepsilon$ given by Lemma 6.22 and $\tilde{C}\left(N, n, C_{0}\right)$ is some constant presented at the end of this proof. Let $\eta<2 \bar{\varepsilon}$ and choose $\varepsilon>0$ such that $\eta \leq 2 \varepsilon<2 \bar{\varepsilon}$. 
Let $a, b \in 2 R_{j} \cap U_{12}$. We obtain using $\sum_{i \in I_{12}} \phi_{i}(a)=1=\sum_{i \in I_{12}} \phi_{i}(b)$

$$
\begin{aligned}
|A(a)-A(b)|= & \left|\sum_{i \in I_{12}} \phi_{i}(a) A_{i}(a)-\sum_{i \in I_{12}} \phi_{i}(b) A_{i}(b)\right| \\
= & \mid \sum_{i \in I_{12}} \phi_{i}(a) A_{i}(a)-\sum_{i \in I_{12}} \phi_{i}(a) A_{i}(b)+\sum_{i \in I_{12}} \phi_{i}(a) A_{i}(b)-\sum_{i \in I_{12}} \phi_{i}(a) A_{j}(b) \\
& +\sum_{i \in I_{12}} \phi_{i}(a) A_{j}(b)-\sum_{i \in I_{12}} \phi_{i}(b) A_{j}(b)+\sum_{i \in I_{12}} \phi_{i}(b) A_{j}(b)-\sum_{i \in I_{12}} \phi_{i}(b) A_{i}(b) \mid \\
\leq & \sum_{i \in I_{12}} \phi_{i}(a)\left|A_{i}(a)-A_{i}(b)\right| \\
& +\left|\sum_{i \in I_{12}} \phi_{i}(a) A_{i}(b)-\sum_{i \in I_{12}} \phi_{i}(a) A_{j}(b)+\sum_{i \in I_{12}} \phi_{i}(b) A_{j}(b)-\sum_{i \in I_{12}} \phi_{i}(b) A_{i}(b)\right| \\
& +\left|\sum_{i \in I_{12}} \phi_{i}(a) A_{j}(b)-\sum_{i \in I_{12}} \phi_{i}(b) A_{j}(b)\right| \\
\leq & \sum_{i \in I_{12}} \phi_{i}(a)\left|A_{i}(a)-A_{i}(b)\right|+\sum_{i \in I_{12}}\left|\phi_{i}(a)-\phi_{i}(b)\right|\left|A_{i}(b)-A_{j}(b)\right| .
\end{aligned}
$$

If $\phi_{i}(a)-\phi_{i}(b) \neq 0$, we get $3 R_{i} \cap 2 R_{j} \neq \emptyset$ and so we can apply Lemma 6.14 (iii), (iv) and Lemma 6.22 (ii). Furthermore, we know from Lemma 6.19 that $A_{i}$ is $2 \alpha$-Lipschitz and we deduce with Lemma 6.20 that $\phi_{i}$ is $\frac{C}{\operatorname{diam} R_{i}}$-Lipschitz. Since $\varepsilon<\bar{\varepsilon} \leq \frac{\alpha}{\tilde{C}}$, we obtain

$$
\begin{aligned}
|A(a)-A(b)| & \leq 2 \alpha|a-b|+(180)^{n} \frac{5 C}{\operatorname{diam} R_{j}}|a-b| \bar{C} \varepsilon \operatorname{diam} R_{j} \\
& =2 \alpha|a-b|+\tilde{C}\left(N, n, C_{0}\right) \varepsilon|a-b| \leq 3 \alpha|a-b| .
\end{aligned}
$$

Lemma 6.24. Under the conditions of the previous lemma for $a, b \in U_{12} \backslash \pi(\mathcal{Z})$ with $[a, b] \subset$ $U_{12} \backslash \pi(\mathcal{Z})$, we have that $d(A(a), A(b)) \leq 3 \alpha d(a, b)$.

Proof. With Lemma 6.14 (ii) it follows that $[a, b] \subset \bigcup_{j \in I_{12}} R_{j}$. Hence, for all $v \in[a, b]$, there exists some $j \in I_{12}$ with $v \in R_{j}$ and, with Lemma 6.14 (i), we get $D(v) \geq 10 \operatorname{diam} R_{j}>0$. Assume that the set $\tilde{I}_{12}:=\left\{i \in I_{12} \mid R_{i} \cap[a, b] \neq \emptyset\right\}$ is infinite. The cubes $R_{i}$ have disjoint interior, so there exists some sequence $\left(R_{i_{l}}\right)_{l \in \mathbb{N}}, i_{l} \in \tilde{I}_{12}$ with diam $R_{i_{l}} \rightarrow 0$. Hence there exists some sequence $\left(v_{l}\right)_{l \in \mathbb{N}}$ with $v_{l} \in R_{i_{l}} \cap[a, b]$ and, with Lemma 6.14 (i), we obtain $D\left(v_{l}\right) \leq 50 \operatorname{diam} R_{i_{l}} \rightarrow 0$. Since $[a, b]$ is compact, $\left(v_{l}\right)_{l \in \mathbb{N}}$ has an accumulation point $\bar{v} \in[a, b]$ and, since $D$ is continuous (Lemma 6.9), we deduce $D(\bar{v})=0$, which is according to Lemma 6.11 equivalent to $\bar{v} \in \pi(\mathcal{Z})$. This is in contradiction to $[a, b] \subset P_{0} \backslash \pi(\mathcal{Z})$ and so the set $\tilde{I}_{12}$ has to be finite.

With Lemma 6.23 and $[a, b] \subset \bigcup_{i \in \tilde{I}_{12}} R_{i}$, we get $d(A(a), A(b)) \leq 3 \alpha d(a, b)$.

Now we show that $A$ is Lipschitz continuous on $U_{12}$ with some large Lipschitz constant. After that, using the continuity of $A$, we are able to prove that $A$ is Lipschitz continuous with Lipschitz constant $3 \alpha$. 
Lemma 6.25. Let $0<\alpha \leq \frac{1}{4}$. There exists some $\bar{k} \geq 4$ and some $\bar{\varepsilon}=\bar{\varepsilon}\left(N, n, C_{0}, \alpha\right)<\alpha$ so that if $k \geq \bar{k}$ and $\eta<2 \bar{\varepsilon}$ for all $\varepsilon \in\left[\frac{\eta}{2}, \bar{\varepsilon}\right), A$ is Lipschitz continuous on $U_{12}$.

Proof. Let $0<\alpha \leq \frac{1}{4}, k \geq \bar{k} \geq 4$, where $\bar{k}$ is the constant from Lemma 6.23, and let $\bar{\varepsilon}=\bar{\varepsilon}\left(N, n, C_{0}, \alpha\right) \leq \frac{\delta}{4}$ be so small that we can apply Lemma 6.12, 6.17, 6.22 and Lemma 6.24. Furthermore, let $\varepsilon>0$ such that $\eta \leq 2 \varepsilon<2 \bar{\varepsilon}$.

Let $a, b \in U_{12}$. We prove $d(A(a), A(b)) \leq C d(a, b)$.

1. Case: $a, b \in \pi(\mathcal{Z})$.

This is already proven in Lemma 6.17.

2. Case: We have $a, b \notin \pi(\mathcal{Z})$ and $[a, b] \subset \bigcup_{j \in I_{12}} 2 R_{j}$.

This is already proven in Lemma 6.24.

3. Case: We have $a \in \pi(\mathcal{Z})$ and there exists some $j \in I_{12}$ with $b \in 2 R_{j}$.

According to Lemma 6.11, we have $D(a)=0$. Since $b \in 2 R_{j}$, we obtain with Lemma 6.14 and because $D$ is 1-Lipschitz (Lemma 6.9)

$$
\operatorname{diam} R_{j} \leq D(b)=D(b)-D(a) \leq d(a, b) .
$$

We estimate $d(A(a), A(b)) \leq d(A(a)+a, A(b)+b) \leq d\left(A(a)+a, X_{j}\right)+d\left(X_{j}, A(b)+b\right)$ where $X_{j}$ is the centre of the ball $B_{j}=B\left(X_{j}, t_{j}\right)$ (see Lemma 6.15).

At first, we consider $d\left(A(a)+a, X_{j}\right)$. By definition of $A$ on $\pi(\mathcal{Z})$, we get $A(a)+a \in \mathcal{Z}$ because $a \in \pi(\mathcal{Z})$. We already know that the function $d \equiv 0$ on $\mathcal{Z}$ (see Lemma 6.11). Hence

$$
d(A(a)+a)=0 .
$$

Moreover, with Lemma 6.15 (i) and $\left(X_{j}, t_{j}\right) \in S$, we deduce

$$
d\left(X_{j}\right)=\inf _{(Y, s) \in S}\left(d\left(Y, X_{j}\right)+s\right) \leq t_{j}=\frac{\operatorname{diam} B_{j}}{2} \leq 100 \operatorname{diam} R_{j} \stackrel{(6.16)}{\leq} 100 d(a, b),
$$

and again by Lemma 6.15 (i), (ii)

$$
d\left(\pi(A(a)+a), \pi\left(X_{j}\right)\right) \leq d(a, b)+d\left(b, \pi\left(X_{j}\right)\right) \leq d(a, b)+C \operatorname{diam} R_{j} \stackrel{(6.16)}{\leq} C d(a, b) .
$$

With Lemma 6.12, we obtain

$$
d\left(A(a)+a, X_{j}\right) \leq 6\left(d(A(a)+a)+d\left(X_{j}\right)\right)+2 d\left(\pi(A(a)+a), \pi\left(X_{j}\right)\right) \leq C d(a, b) .
$$

Now we consider $d\left(X_{j}, A(b)+b\right)$. We have $\left(X_{j}, t_{j}\right) \in S \subset S_{\text {total }}$ and hence, with Lemma 5.10 (ii) $\left(\sigma=2 \varepsilon, x=X_{j}, t=t_{j}, \lambda=\frac{\delta}{2}, P=P_{j}\right)$ using $\varepsilon<\bar{\varepsilon} \leq \frac{\delta}{4}$, there exists some $y \in B\left(X_{j}, 2 t_{j}\right) \cap P_{j}$, where $P_{j}$ is the associated plane to $B_{j}$ (see Remark 6.3 (iii)). We conclude

$$
d\left(X_{j}, A(b)+b\right) \leq d\left(X_{j}, y\right)+d\left(y, A_{j}(b)+b\right)+d\left(A_{j}(b)+b, A(b)+b\right) .
$$

With Lemma 6.22 (iv) and $\varepsilon \leq \bar{\varepsilon}<1$, there exists some $C=C\left(N, n, C_{0}\right)$ so that

$$
d\left(A_{j}(b)+b, A(b)+b\right)=d\left(A(b), A_{j}(b)\right) \leq C \varepsilon \operatorname{diam} R_{j} \leq C d(a, b),
$$


so that, with $y \in 2 B_{j} \cap P_{j}$ and $\Varangle\left(P_{j}, P_{0}\right) \leq \alpha \leq \frac{1}{4}$, we deduce with Lemma 2.23 and Lemma 6.15 (i), (ii)

$$
\begin{aligned}
d\left(X_{j}, A(b)+b\right) & \leq d\left(X_{j}, y\right)+\overbrace{d(y, \underbrace{\left.A_{j}(b)+b\right)}_{\in P_{j}}}^{\leq \frac{1}{1-\alpha} d(\pi(y), b)}+d\left(A_{j}(b)+b, A(b)+b\right) \\
& \leq \operatorname{diam} B_{j}+2 d(\pi(y), b)+C d(a, b) \\
& \stackrel{(6.16)}{\leq} C d(a, b) .
\end{aligned}
$$

4. Case: We have $a, b \notin \pi(\mathcal{Z})$ and there exists some $d \in[a, b] \cap \pi(\mathcal{Z})$.

We get using case 3

$$
d(A(a), A(b)) \leq d(A(a), A(d))+d(A(d), A(b)) \leq C d(a, d)+C d(d, b)=C d(a, b) .
$$

Lemma 6.26. Under the conditions of Lemma 6.25 for some $a \in \pi(\mathcal{Z}), i \in I_{12}$ and $b \in 2 R_{j}$, we get

$$
d(A(a), A(b)) \leq 3 \alpha d(a, b)
$$

Proof. We set $c:=\inf _{x \in[a, b] \cap \pi(\mathcal{Z})} d(x, b)$. Since $\pi(\mathcal{Z})$ is closed (see Lemma 6.11), there exists some $v \in[a, b] \cap \pi(\mathcal{Z})$ with $d(v, b)=c$. Furthermore, there exists some sequence $\left(v_{l}\right)_{l} \subset[v, b]$ with $v_{l} \rightarrow v$ where $l \rightarrow \infty$. With Lemma 6.14 , we deduce $([v, b] \backslash\{v\}) \subset \bigcup_{j \in I_{12}} 2 R_{j}$. For every $l \in \mathbb{N}$ we obtain with Lemma 6.24

$$
\begin{aligned}
d(A(v), A(b)) & \leq d\left(A(v), A\left(v_{l}\right)\right)+d\left(A\left(v_{l}\right), A(b)\right) \\
& \leq d\left(A(v), A\left(v_{l}\right)\right)+3 \alpha d\left(v_{l}, b\right) \\
& \leq d\left(A(v), A\left(v_{l}\right)\right)+3 \alpha d(v, b) .
\end{aligned}
$$

and, since $A$ is continuous (Lemma 6.25) we conclude with $l \rightarrow \infty$

$$
d(A(v), A(b)) \leq 3 \alpha d(v, b)
$$

We already know that $A$ is $2 \alpha$-Lipschitz on $\pi(\mathcal{Z})$ so we get since $v \in[a, b]$

$$
\begin{aligned}
d(A(a), A(b)) & \leq d(A(a), A(v)))+d(A(v), A(b)) \\
& \leq 2 \alpha d(a, v)+3 \alpha d(v, b) \\
& \leq 3 \alpha d(a, b) .
\end{aligned}
$$

Lemma 6.27. Under the conditions of Lemma 6.25 for $a, b \in \bigcup_{j \in I_{12}} 2 R_{j} \cap U_{12}$, we have

$$
d(A(a), A(b)) \leq 3 \alpha d(a, b) .
$$


Proof. Let $a \in 2 R_{i}$ and $b \in 2 R_{j}$. If $i=j$, the statement is already proven in Lemma 6.23. So we assume $i \neq j$.

1. Case: There exists some $v \in \pi(\mathcal{Z})$ with $v \in[a, b]$.

We obtain with Lemma 6.26

$$
\begin{aligned}
d(A(a), A(b)) & \leq d(A(a), A(v))+d(A(v), A(b)) \\
& \leq 3 \alpha d(a, v)+3 \alpha d(v, b) \\
& =3 \alpha d(a, b) .
\end{aligned}
$$

2. Case: We have $[a, b] \subset U_{12} \backslash \pi(\mathcal{Z})$.

This is already proven in Lemma 6.24.

Lemma 6.28. Under the conditions of Lemma 6.25, the function A is Lipschitz continuous on $U_{12}$ with Lipschitz constant $3 \alpha$.

Proof. With Lemma 6.17 we already know that $A$ is $2 \alpha$-Lipschitz on $\pi(\mathcal{Z})$. Hence the previous lemmas imply that $A$ is Lipschitz continuous on $U_{12}=\left(U_{12} \cap \bigcup_{j \in I_{12}} 2 R_{j}\right) \cup \dot{U} \pi(\mathcal{Z})$ with Lipschitz constant $3 \alpha$.

The following estimate is for later use.

Lemma 6.29. Let $0<\alpha \leq \frac{1}{4}$. There exists some $\bar{k} \geq 4$ and some $\bar{\varepsilon}=\bar{\varepsilon}\left(N, n, C_{0}\right)$ so that if $k \geq \bar{k}$ and $\eta<2 \bar{\varepsilon}$ for all $\varepsilon \in\left[\frac{\eta}{2}, \bar{\varepsilon}\right)$, there exists some constant $C=C\left(N, n, C_{0}\right)$ so that for all $j \in I_{12}$ and for all multi-indices $\kappa$ with $|\kappa|=2$ and $a \in 2 R_{j}$

$$
\left|\partial^{\kappa} A(a)\right| \leq \frac{C \varepsilon}{\operatorname{diam} R_{j}} .
$$

Proof. Choose $\bar{k}$ and $\bar{\varepsilon}$ as in Lemma 6.22. Let $\kappa$ be some multi-index with $|\kappa|=2$. For $i \in I_{12}$, the function $A_{i}$ is an affine map, so $\partial^{\kappa} A_{i}=0$ and hence for some suitable $l_{1}, l_{2} \in\{1, \ldots, n\}$ we have

$$
\partial^{\kappa} A=\partial^{\kappa}\left(\sum_{i \in I_{12}} \phi_{i} A_{i}\right)=\sum_{i \in I_{12}}\left(\partial^{\kappa} \phi_{i}\right) A_{i}+\sum_{i \in I_{12}}\left(\partial_{l_{1}} \phi_{i} \partial_{l_{2}} A_{i}+\partial_{l_{2}} \phi_{i} \partial_{l_{1}} A_{i}\right) .
$$

Let $j \in I_{12}$ and $a \in 2 R_{j}$. Since $\phi_{i} \equiv 0$ on the exterior of $3 R_{i}$ where $i \in I_{12}$, with Lemma 6.14 , there exist at most $180^{n}$ cells $R_{i}$ so that $\partial^{\kappa} \phi_{i}(a) \neq 0$ or $\partial^{\omega} \phi_{i}(a) \neq 0$, where $\omega$ is a multi-index with $|\omega|=1$. So only finite sums occur in the following estimates. We have $\sum_{i \in I_{12}} \partial^{\omega} \phi_{i}=\partial^{\omega} \sum_{i \in I_{12}} \phi_{i}=\partial^{\omega} 1=0$ so that, for $a \in 2 R_{j}$, we get

$$
\begin{aligned}
& \left|\partial^{\kappa} A(a)\right| \\
\stackrel{(6.17)}{\leq} \mid & \sum_{i \in I_{12}}\left(\partial^{\kappa} \phi_{i}(a)\right) A_{i}(a)-\sum_{i \in I_{12}}\left(\partial^{\kappa} \phi_{i}(a)\right) A_{j}(a) \mid \\
+ & \left|\sum_{i \in I_{12}}\left(\partial_{l_{1}} \phi_{i}(a) \partial_{l_{2}} A_{i}(a)+\partial_{l_{2}} \phi_{i}(a) \partial_{l_{1}} A_{i}(a)\right)-\sum_{i \in I_{12}}\left(\partial_{l_{1}} \phi_{i}(a) \partial_{l_{2}} A_{j}(a)+\partial_{l_{2}} \phi_{i}(a) \partial_{l_{1}} A_{j}(a)\right)\right| \\
\leq & \sum_{i \in I_{12}}\left|\partial^{\kappa} \phi_{i}(a)\right|\left|A_{i}(a)-A_{j}(a)\right| \\
+ & \sum_{i \in I_{12}}\left|\partial_{l_{1}} \phi_{i}(a)\right|\left|\partial_{l_{2}}\left(A_{i}(a)-A_{j}(a)\right)\right|+\sum_{i \in I_{12}}\left|\partial_{l_{2}} \phi_{i}(a)\right|\left|\partial_{l_{1}}\left(A_{i}(a)-A_{j}(a)\right)\right| .
\end{aligned}
$$


To estimate these sums, we only have to consider the case when $a$ is in the support of $\phi_{i}$ for some $i \in I_{12}$. This implies $3 R_{i} \cap 2 R_{j} \neq \emptyset$ and so we can apply Lemma 6.22. For $a \in 3 R_{i} \cap 2 R_{j}$, we have the following estimates (see Lemma 6.22 and 6.20)

$$
\begin{aligned}
\left|A_{i}(a)-A_{j}(a)\right| & \leq C\left(N, n, C_{0}\right) \varepsilon \operatorname{diam} R_{j}, \\
\left|\partial^{\omega}\left(A_{i}(a)-A_{j}(a)\right)\right| & \leq C\left(N, n, C_{0}\right) \varepsilon, \\
\left|\partial^{\omega} \phi_{i}(a)\right| & \leq \frac{C(n)}{\operatorname{diam} R_{i}}, \\
\left|\partial^{\kappa} \phi_{i}(a)\right| & \leq \frac{C(n)}{\left(\operatorname{diam} R_{i}\right)^{2}},
\end{aligned}
$$

and hence, with Lemma 6.14 (iii), (iv), we obtain

$$
\left|\partial^{\kappa} A(a)\right| \leq 180^{n} \frac{C(n)}{\left(\operatorname{diam} R_{j}\right)^{2}} C \varepsilon \operatorname{diam} R_{j}+2 \cdot 180^{n} \frac{C(n)}{\operatorname{diam} R_{j}} C \varepsilon \leq \frac{C \varepsilon}{\operatorname{diam} R_{j}} .
$$





\section{$7 \gamma$-functions}

In this chapter, we introduce the $\gamma$-function of some function $g: P_{0} \rightarrow P_{0}^{\perp}$. This function measures how well $g$ can be approximated in some ball by some affine function. The main results of this chapter are Theorem 7.3 on page 90 and Theorem 7.17 on page 103. We will use these statements in section 8.4 to prove that $\mu\left(F_{3}\right)$ is small.

Definition 7.1. Let $U \subset P_{0}, q \in U$ and $t>0$ so that $B(q, t) \cap P_{0} \subset U$. Furthermore, let $\mathcal{A}=\mathcal{A}\left(P_{0}, P_{0}^{\perp}\right)$ be the set of all affine functions $a: P_{0} \rightarrow P_{0}^{\perp}, \mathcal{P}(N, n)$ the set of all $n$-dimensional affine planes in $\mathbb{R}^{N}$ and let $g: U \rightarrow P_{0}^{\perp}$ be some function. We define

$$
\gamma_{g}(q, t):=\inf _{a \in \mathcal{A}} \frac{1}{t^{n}} \int_{B(q, t) \cap P_{0}} \frac{d(g(u), a(u))}{t} \mathrm{~d} \mathcal{H}^{n}(u)
$$

and

$$
\tilde{\gamma}_{g}(q, t):=\inf _{P \in \mathcal{P}(N, n)} \frac{1}{t^{n}} \int_{B(q, t) \cap P_{0}} \frac{d(u+g(u), P)}{t} \mathrm{~d} \mathcal{H}^{n}(u) .
$$

Lemma 7.2. Let $U \subset P_{0}, q \in U$ and $t>0$ so that $B(q, t) \cap P_{0} \subset U$. Furthermore, let $g: U \rightarrow P_{0}^{\perp}$ be a Lipschitz continuous function with Lipschitz constant

$\operatorname{Lip}_{g} \leq\left[60 n\left(10^{n}+1\right) 2^{n}\left(n \frac{4 \omega_{n-1}}{\omega_{n}}\right)^{n+1}\right]^{-1}$, where $\omega_{n}$ denotes the $n$-dimensional volume of the $n$-dimensional unit ball. Then we have

$$
\tilde{\gamma}_{g}(q, t) \leq \gamma_{g}(q, t) \leq 3 \tilde{\gamma}_{g}(q, t)
$$

Proof. Let $g$ be a Lipschitz continuous function with an appropriate Lipschitz constant. For every $a \in \mathcal{A}$ the graph $G(a)$ of $a$ is in $\mathcal{P}(N, n)$. We obtain

$$
\begin{aligned}
\tilde{\gamma}_{g}(q, t) & =\inf _{P \in \mathcal{P}(N, n)} \frac{1}{t^{n}} \int_{B(q, t) \cap P_{0}} \frac{d(u+g(u), P)}{t} \mathrm{~d} \mathcal{H}^{n}(u) \\
& \leq \inf _{a \in \mathcal{A}} \frac{1}{t^{n}} \int_{B(q, t) \cap P_{0}} \frac{d(u+g(u), G(a))}{t} \mathrm{~d} \mathcal{H}^{n}(u) \\
& \leq \inf _{a \in \mathcal{A}} \frac{1}{t^{n}} \int_{B(q, t) \cap P_{0}} \frac{d(u+g(u), u+a(u))}{t} \mathrm{~d} \mathcal{H}^{n}(u) \\
& =\inf _{a \in \mathcal{A}} \frac{1}{t^{n}} \int_{B(q, t) \cap P_{0}} \frac{d(g(u), a(u))}{t} \mathrm{~d} \mathcal{H}^{n}(u) \\
& =\gamma_{g}(q, t),
\end{aligned}
$$


which is the first inequality. Now we treat the second one. We deduce

$$
\begin{aligned}
\gamma_{g}(q, t) & =\inf _{a \in \mathcal{A}} \frac{1}{t^{n}} \int_{B(q, t) \cap P_{0}} \frac{d(g(u), a(u))}{t} \mathrm{~d} \mathcal{H}^{n}(u) \\
& \leq \frac{1}{t^{n+1}} \int_{B(q, t) \cap P_{0}} \underbrace{d(g(u), g(q))}_{\leq \operatorname{Lip}_{g} d(u, q) \leq \operatorname{Lip}_{g} t} \mathrm{~d} \mathcal{H}^{n}(u) \\
& \leq \frac{1}{t^{n+1}} \operatorname{Lip}_{g} t \underbrace{\mathcal{H}^{n}\left(B(q, t) \cap P_{0}\right)}_{=\omega_{n} t^{n}} \\
& \leq \operatorname{Lip}_{g} \omega_{n} .
\end{aligned}
$$

The first inequality implies

$$
\tilde{\gamma}_{g}(q, t) \leq \gamma_{g}(q, t) \leq \operatorname{Lip}_{g} \omega_{n}
$$

Let $0<\xi<\operatorname{Lip}_{g} \omega_{n}$ and choose some $P \in \mathcal{P}(N, n)$ so that

$$
\frac{1}{t^{n}} \int_{B(q, t) \cap P_{0}} \frac{d(u+g(u), P)}{t} \mathrm{~d} \mathcal{H}^{n}(u) \leq \tilde{\gamma}_{g}(q, t)+\xi \leq 2 \operatorname{Lip}_{g} \omega_{n}
$$

We obtain

$$
\begin{aligned}
& \mathcal{H}^{n}(\underbrace{\left\{v \in B(q, t) \cap P_{0} \mid d(v+g(v), P) \leq 4 \operatorname{Lip}_{g} t\right\}}_{=: D_{1}}) \\
& \geq \mathcal{H}^{n}\left(B(q, t) \cap P_{0}\right)-\mathcal{H}^{n}(\underbrace{\left\{v \in B(q, t) \cap P_{0} \mid d(v+g(v), P)>4 \operatorname{Lip}_{g} t\right\}}_{=: D_{2}}) \\
& =\omega_{n} t^{n}-\frac{1}{4 \operatorname{Lip}_{g} t} \int_{D_{2}} 4 \operatorname{Lip}_{g} t \mathrm{~d} \mathcal{H}^{n}(u) \\
& >\omega_{n} t^{n}-\frac{1}{4 \operatorname{Lip}_{g} t} \int_{D_{2}} d(u+g(u), P) \mathrm{d} \mathcal{H}^{n}(u)
\end{aligned}
$$

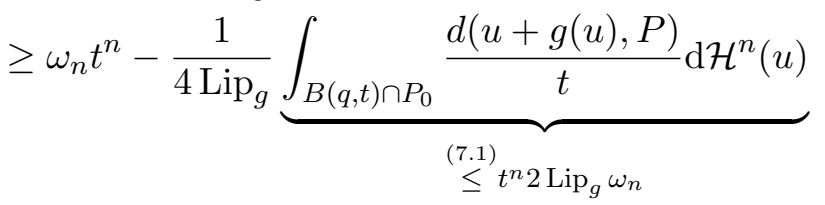

$$
\begin{aligned}
& \geq \frac{\omega_{n}}{2} t^{n} .
\end{aligned}
$$

Assume that every simplex $\triangle\left(u_{0}, \ldots, u_{n}\right) \in D_{1}$ is not an $(n, H)$-simplex, where $H=\frac{\omega_{n}}{4 \omega_{n-1}} t$.

With Lemma $2.16\left(m=n, D=D_{1}\right)$, there exists some plane $\hat{P} \in \mathcal{P}(N, n-1)$ with

$$
D_{1} \subset U_{H}(\hat{P}) \cap B(q, t) \cap P_{0} .
$$

We get

$$
\mathcal{H}^{n}\left(D_{1}\right) \leq \mathcal{H}^{n}\left(U_{H}(\hat{P}) \cap B(q, t) \cap P_{0}\right) \leq 2 H \omega_{n-1} t^{n-1}=\frac{\omega_{n}}{2} t^{n}
$$


This is in contradiction to (7.2), so there exists some $(n, H)$-simplex $\triangle\left(u_{0}, \ldots, u_{n}\right) \in D_{1}$. We set $\hat{P}_{0}:=P_{0}+g\left(u_{0}\right), y_{i}:=u_{i}+g\left(u_{0}\right) \in \hat{P}_{0}$ for all $i \in\{0, \ldots, n\}$ and $S:=\Delta\left(y_{0}, \ldots, y_{n}\right) \subset \hat{P}_{0} \cap$ $B\left(q+g\left(u_{0}\right), t\right)$. We recall that $P$ is the plane satisfying (7.1). We obtain for all $i \in\{0, \ldots, n\}$

$$
d\left(y_{i}, P\right) \leq d\left(u_{i}+g\left(u_{0}\right), u_{i}+g\left(u_{i}\right)\right)+d\left(u_{i}+g\left(u_{i}\right), P\right) \leq \operatorname{Lip}_{g} d\left(u_{0}, u_{i}\right)+4 \operatorname{Lip}_{g} t \leq 6 \operatorname{Lip}_{g} t .
$$

With Lemma 2.28, $C=\frac{4 \omega_{n-1}}{\omega_{n}}>1^{1}, \hat{C}=1, m=n, \sigma=6 \operatorname{Lip}_{g}, P_{1}=\hat{P}_{0}, P_{2}=P$ and $x=q+g\left(u_{0}\right)$, we get

$$
\Varangle\left(P_{0}, P\right)=\Varangle\left(\hat{P}_{0}, P\right) \leq 4 n\left(10^{n}+1\right) 2^{n}\left(n \frac{4 \omega_{n-1}}{\omega_{n}}\right)^{n+1} 6 \operatorname{Lip}_{g}<\frac{1}{2},
$$

and, with Corollary 2.25, there exists some affine map $\bar{a}: P_{0} \rightarrow P_{0}^{\perp}$ with graph $G(\bar{a})=P$. Now we obtain with Corollary $2.24\left(P_{1}=P, P_{2}=P_{0}\right), u, v \in P_{0}$ and $\Varangle\left(P_{0}, P\right)<\frac{1}{2}$

$$
\begin{aligned}
d(\underbrace{v+\bar{a}(v)}_{\in P}, \underbrace{u+\bar{a}(u)}_{\in P}) & \leq 2 d\left(\pi_{P_{0}}(v+\bar{a}(v)), \pi_{P_{0}}(u+\bar{a}(u))\right) \\
& =2 d\left(\pi_{P_{0}}(v+\bar{a}(v)), \pi_{P_{0}}(u+g(u))\right) .
\end{aligned}
$$

That yields for $u \in B(q, t) \cap P_{0}$ and some suitable $v \in P_{0}$

$$
\begin{aligned}
d(g(u), \bar{a}(u)) & =d(u+g(u), u+\bar{a}(u)) \\
& \leq d(u+g(u), P)+d(\underbrace{\pi_{P}(u+g(u))}_{=: v+\bar{a}(v)}, u+\bar{a}(u)) \\
& \stackrel{(7.3)}{\leq} d(u+g(u), P)+2 d\left(\pi_{P_{0}}(\underbrace{v+\bar{a}(v)}_{=\pi_{P}(u+g(u))}), \pi_{P_{0}}(u+g(u))\right) \\
& \leq d(u+g(u), P)+2 \underbrace{d\left(\pi_{P}(u+g(u)), u+g(u)\right)}_{=d(u+g(u), P)} \\
& =3 d(u+g(u), P) .
\end{aligned}
$$

Finally, we get

$$
\begin{aligned}
\gamma_{g}(q, t) & =\inf _{a \in \mathcal{A}} \frac{1}{t^{n}} \int_{B(q, t) \cap P_{0}} \frac{d(g(u), a(u))}{t} \mathrm{~d} \mathcal{H}^{n}(u) \\
& \leq \frac{1}{t^{n}} \int_{B(q, t) \cap P_{0}} \frac{d(g(u), \bar{a}(u))}{t} \mathrm{~d} \mathcal{H}^{n}(u) \\
& \leq 3 \frac{1}{t^{n}} \int_{B(q, t) \cap P_{0}} \frac{d(u+g(u), P)}{t} \mathrm{~d} \mathcal{H}^{n}(u) \\
& \stackrel{(7.1)}{\leq} 3\left(\tilde{\gamma}_{g}(q, t)+\xi\right),
\end{aligned}
$$

and $0<\xi<\alpha \omega_{n}$ was arbitrarily chosen.

\footnotetext{
${ }^{1}$ As the volume of the unit sphere is strictly monotonously decreasing when the dimension $n \geq 5$ increases, we get $\frac{\omega_{n-1}}{\omega_{n}}>1$ for all $n \geq 6$. With the factor 4 we have that $4 \frac{\omega_{n-1}}{\omega_{n}}>1$ for all $n \in \mathbb{N}$.
} 


\section{1 $\gamma$-functions and affine approximation of Lipschitz functions}

In this section, we use the notation $U_{l}:=B(0, l) \cap P_{0}$ for $l \in\{6,8,10\}$.

Theorem 7.3. Let $1<p<\infty$ and let $g: P_{0} \rightarrow P_{0}^{\perp}$ be a Lipschitz continuous function with Lipschitz constant $\operatorname{Lip}_{g}$ and compact support. For all $\theta>0$, there exists some set $H_{\theta} \subset U_{6}$ and some constants $C=C(n, p)$ and $\hat{C}=\hat{C}(n, N)$ with

$$
\mathcal{H}^{n}\left(U_{6} \backslash H_{\theta}\right) \leq \frac{C}{\theta^{p(n+1)} \operatorname{Lip}_{g}^{p}} \int_{U_{10}}\left(\int_{0}^{2} \gamma_{g}(x, t)^{2} \frac{\mathrm{d} t}{t}\right)^{\frac{p}{2}} \mathrm{~d} \mathcal{H}^{n}(x)
$$

so that, for all $y \in P_{0}$, there exists some affine map $a_{y}: P_{0} \rightarrow P_{0}^{\perp}$ so that if $r \leq \theta$ and $B(y, r) \cap H_{\theta} \neq \emptyset$, we have

$$
\left\|g-a_{y}\right\|_{L^{\infty}\left(B(y, r) \cap P_{0}, P_{0}^{\perp}\right)} \leq \hat{C} r \theta \operatorname{Lip}_{g},
$$

where $\|\cdot\|_{L^{\infty}(E)}$ denotes the essential supremum on $E \subset P_{0}$ with respect to the $\mathcal{H}^{n}$-measure.

To prove this theorem, we need the following lemma. If $\nu$ is some map, we use the notation $\nu_{t}(x):=\frac{1}{t^{n}} \nu\left(\frac{x}{t}\right)$.

Lemma 7.4. There exists some radial function $\nu \in C_{0}^{\infty}\left(P_{0}, \mathbb{R}\right)$ with

1. $\operatorname{supp}(\nu) \subset B(0,1) \cap P_{0}$ and $\widehat{\nu}(0)=0$,

2. there exists some constant $c>0$ so that for all $x \in P_{0} \backslash\{0\}$ and $i \in\{1, \ldots, n\}$, we have

$$
\int_{0}^{\infty}|\widehat{\nu}(t x)|^{2} \frac{\mathrm{d} t}{t}=1 \quad \text { and } \quad 0<\int_{0}^{\infty}\left|\widehat{\left(\partial_{i} \nu\right)_{t}}(x)\right|^{2} \frac{\mathrm{d} t}{t} \leq c<\infty
$$

3. for all $i \in\{1, \ldots, n\}$, the function $\partial_{i} \nu$ has mean value zero and, for all $a \in \mathcal{A}\left(P_{0}, P_{0}^{\perp}\right)$ (affine functions), we have

$$
\int_{P_{0}} a \nu \mathrm{d} \mathcal{H}^{n}=0
$$

4. for all $w \in P_{0}$ and $i \in\{1, \ldots, n\}$, we have

$$
\partial_{i} \nu(-w)=-\partial_{i} \nu(w),
$$

5. $\widehat{\nu}$ is radial.

Proof. Let

$$
\nu_{1}: P_{0} \rightarrow \mathbb{R}
$$

be some non harmonic $\left(\Delta \nu_{1} \neq 0\right)$, radial $C^{\infty}$ function with support in $B(0,1) \cap P_{0}$. We set $\nu_{2}:=\Delta \nu_{1} \in C^{\infty}\left(P_{0}\right) \cap C_{0}^{\infty}\left(B(0,1) \cap P_{0}\right)$ and $0<c_{1}:=\int_{0}^{\infty}\left|\widehat{\nu_{2}}(t e)\right|^{2} \frac{\mathrm{d} t}{t}$, where $e$ is some normed vector in $P_{0}$. With Lemma A.22, we get $\nu_{2}$ is radial as well. Using Lemma A.20, we obtain

$$
\left|\widehat{\nu_{2}}(t e)\right|=\left|\sum_{j=1}^{n} \widehat{\partial_{j} \partial_{j} \nu_{1}}(t e)\right|=(2 \pi)^{2}\left|\widehat{\nu_{1}}(t e)\right| \sum_{j=1}^{n} \phi^{-1}(t e)_{j}^{2}=4 \pi^{2} t^{2}\left|\widehat{\nu_{1}}(t e)\right|
$$


and hence

$$
0<c_{1}=\int_{0}^{\infty}\left|\widehat{\nu_{2}}(t e)\right|^{2} \frac{\mathrm{d} t}{t}=16 \pi^{4} \int_{0}^{\infty} t^{3}\left|\widehat{\nu_{1}}(t e)\right|^{2} \mathrm{~d} t<\infty
$$

because $\nu_{1}$ is in the Schwarz space and therefore $\widehat{\nu}_{1}$ as well [Gra08, 2.2.15, 2.2.11 (11)]. The previous equality also implies $\widehat{\nu_{2}}(0)=0$. Now we set

$$
\nu:=\sqrt{\frac{1}{c_{1}}} \nu_{2}
$$

which is a radial $C_{0}^{\infty}\left(P_{0}, \mathbb{R}\right)$ function that fulfils 1 . With [Gra08, 2.2.12], we get $\widehat{\nu}$ is radial, so it fulfils 5 . We have for all $x \in P_{0} \backslash\{0\}$ (use substitution with $t=r \frac{1}{|x|}$ and the fact that $\widehat{\nu}$ is radial)

$$
\int_{0}^{\infty}|\widehat{\nu}(t x)|^{2} \frac{\mathrm{d} t}{t}=\int_{0}^{\infty}|\widehat{\nu}(r e)|^{2} \frac{\mathrm{d} r}{r}=1 .
$$

In a similar way, we deduce for $i \in\{1, \ldots, n\}$ with Lemma A.20 (using $\left|\left(\phi^{-1}(t x)\right)^{\kappa}\right| \leq$ $\left|\phi^{-1}(t x)\right|=|t x|$ where $\kappa$ is some multi-index with $\left.|\kappa|=1\right)$

$$
\left.\int_{0}^{\infty} \widehat{\mid\left(\partial_{i} \nu\right)_{t}}(x)\right|^{2} \frac{\mathrm{d} t}{t} \leq 4 \pi^{2} \int_{0}^{\infty}|t x|^{2}|\widehat{\nu}(t x)|^{2} \frac{\mathrm{d} t}{t}=4 \pi^{2} \int_{0}^{\infty} r\left|\widehat{\nu}\left(r \frac{x}{|x|}\right)\right|^{2} \mathrm{~d} r<\infty,
$$

where we use that the Fourier transform of a Schwartz function is a Schwartz function as well [Gra08, 2.2.15]. The left hand side of the previous inequality can not be zero, because this would implicate that $\partial_{i} \nu(x)=0$ for all $x \in P_{0}$, which is in contradiction to $0 \neq \nu \in C_{0}^{\infty}\left(P_{0}, \mathbb{R}\right)$. Hence $\nu$ fulfils 2 .

Now we get for all $a \in \mathcal{A}\left(P_{0}, P_{0}^{\perp}\right)$ (see Lemma A.17, partial integration)

$$
\int_{P_{0}} a \nu \mathrm{d} \mathcal{H}^{n}=\int_{P_{0}} a \sqrt{\frac{1}{c_{1}}} \Delta \nu_{1} \mathrm{~d} \mathcal{H}^{n}=\int_{P_{0}} \underbrace{\Delta a}_{=0} \sqrt{\frac{1}{c_{1}}} \nu_{1} \mathrm{~d} \mathcal{H}^{n}=0,
$$

and the same argument implies that $\partial_{i} \nu$ has mean value zero. At last, we show that $\nu$ fulfils (7.6). For $i \in\{1, \ldots, n\}$, there exists some $\tilde{e} \in \mathbb{R}^{n}$ so that we get with Definition A.13, using that $\nu$ is radial,

$$
\begin{aligned}
\partial_{i} \nu(-w) & =\lim _{h \rightarrow 0} \frac{(\nu \circ \phi)\left(\phi^{-1}(-w)+h \tilde{e}\right)-\nu(-w)}{h} \\
& =-\lim _{h \rightarrow 0} \frac{(\nu \circ \phi)\left(\phi^{-1}(w)+(-h) \tilde{e}\right)-\nu(w)}{-h}=-\partial_{i} \nu(w) .
\end{aligned}
$$

For some function $f: P_{0} \rightarrow P_{0}^{\perp}$ and $x \in P_{0}$, we define the convolution of $\nu_{t}$ and $f$ by

$$
\left(\nu_{t} * f\right)(x):=\int_{P_{0}} \nu_{t}(x-y) f(y) \mathrm{d} \mathcal{H}^{n}(y) .
$$


Lemma 7.5 (Calderón's identity). Let $\nu$ be the function given by Lemma 7.4 and let $u \in$ $P_{0} \backslash\{0\}$ and $f \in L^{2}\left(P_{0}, P_{0}^{\perp}\right)$ or let $f \in \mathscr{S}^{\prime}\left(P_{0}\right)$ be a tempered distribution and $u \in \mathscr{S}\left(P_{0}\right)$ (Schwartz space) with $u(0)=0$. Then we have

$$
f(u)=\int_{0}^{\infty}\left(\nu_{t} * \nu_{t} * f\right)(u) \frac{\mathrm{d} t}{t} .
$$

Remark. Léger calls this identity "Calderón's formula" [Lég99, p. 862, 5. Calderón's formula and the size of $F_{3}$ ]. Grafakos presents a similar version called "Calderón reproducing formula" [Gra08, p.371, Exercise 5.2.2].

Proof. At first, let $f \in L^{2}\left(P_{0}, P_{0}^{\perp}\right)$ and $u \in P_{0} \backslash\{0\}$. We have with Lemma A.20 $\widehat{\left(\nu_{t}\right)}(u)=$ $\widehat{\nu}(t u)$ and, with Fubini's theorem, Lemma A.19 and [Gra08, 2.2.4 The Fourier Transform on $\left.L^{1}+L^{2}\right]$, we obtain with $(7.4)$

$$
\begin{aligned}
& \left(\int_{0}^{\infty}\left(\nu_{t} * \nu_{t} * f\right)(u) \frac{\mathrm{d} t}{t}\right)^{\wedge}=\int_{0}^{\infty}\left(\nu_{t} * \nu_{t} * f\right) \hat{(u)} \frac{\mathrm{d} t}{t} \\
& =\int_{0}^{\infty} \widehat{\left(\nu_{t}\right)}(u) \widehat{\left(\nu_{t}\right)}(u) \widehat{f}(u) \frac{\mathrm{d} t}{t} \\
& =\widehat{f}(u) \underbrace{\int_{0}^{\infty}|\widehat{\nu}(t u)|^{2} \frac{\mathrm{d} t}{t}}_{=1} \\
& =\widehat{f}(u) .
\end{aligned}
$$

The Fourier inversion holds on $L^{2}\left(P_{0}, P_{0}^{\perp}\right)$ [Gra08, 2.2.4 The Fourier Transform on $L^{1}+L^{2}$, which gives the statement.

Now let $f$ be a tempered distribution and $u \in \mathscr{S}\left(P_{0}\right)$ with $u(0)=0$. Using Lemma 7.4 1 . and 2., we deduce for all $x \in P_{0}$ that

$$
u(x)=u(x) \int_{0}^{\infty} \widehat{\left(\nu_{t}\right)}(x) \widehat{\left(\nu_{t}\right)}(x) \frac{\mathrm{d} t}{t}
$$

and hence we get with [Gra08, Prop. 2.3.22] and [Yos80, Chapter V, Section 5, Cor. 2]

$$
\begin{aligned}
& \left(\int_{0}^{\infty}\left(\nu_{t} * \nu_{t} * f\right)(u) \frac{\mathrm{d} t}{t}\right)^{-}=\int_{0}^{\infty}\left(\nu_{t} * \nu_{t} * f\right)(\widehat{u}) \frac{\mathrm{d} t}{t} \\
& =\int_{0}^{\infty}\left(\nu_{t} * \nu_{t} * f\right) \hat{(u)} \frac{\mathrm{d} t}{t} \\
& =\int_{0}^{\infty}\left(\widehat{\left(\nu_{t}\right)} \widehat{\left(\nu_{t}\right)} \widehat{f}\right)(u) \frac{\mathrm{d} t}{t} \\
& =\int_{0}^{\infty} \widehat{f}\left(\widehat{\left(\nu_{t}\right)} \widehat{\left(\nu_{t}\right)} u\right) \frac{\mathrm{d} t}{t} \\
& =\widehat{f}\left(u \int_{0}^{\infty} \widehat{\left(\nu_{t}\right)} \widehat{\left(\nu_{t}\right)} \frac{\mathrm{d} t}{t}\right) \\
& =\widehat{f}(u) \text {. }
\end{aligned}
$$


Proof of Theorem 7.3. Let $g \in C_{0}^{0,1}\left(P_{0}, P_{0}^{\perp}\right)$ and let $\nu$ be the function given by Lemma 7.4. We apply the previous lemma on $g \in L^{2}\left(P_{0}, P_{0}^{\perp}\right)$ and get for $u \in P_{0} \backslash\{0\}$

$$
g(u)=\int_{0}^{\infty}\left(\nu_{t} * \nu_{t} * g\right)(u) \frac{\mathrm{d} t}{t} .
$$

We define

$$
\begin{aligned}
& g_{1}(u):=\int_{2}^{\infty}\left(\nu_{t} * \nu_{t} * g\right)(u) \frac{\mathrm{d} t}{t}+\int_{0}^{2}\left(\nu_{t} *\left(\mathbb{1}_{P_{0} \backslash U_{10}} \cdot\left(\nu_{t} * g\right)\right)\right)(u) \frac{\mathrm{d} t}{t}, \\
& g_{2}(u):=\int_{0}^{2}\left(\nu_{t} *\left(\mathbb{1}_{U_{10}} \cdot\left(\nu_{t} * g\right)\right)\right)(u) \frac{\mathrm{d} t}{t} .
\end{aligned}
$$

The convolution fulfils the distributive property [Gra08, Prop 1.2.9, (2)], so we get $g=g_{1}+g_{2}$. We recall the notation $U_{l}=B(0, l) \cap P_{0}$ for $l \in\{6,8,10\}$ and continue the proof of Theorem 7.3 with several lemmas.

\section{Lemma 7.6.}

1. $g_{1} \in C^{\infty}\left(U_{8}\right)$ and there exists some constant $C=C(\nu)$ so that for all multi-indices $\kappa$ with $|\kappa| \leq 2$

$$
\left\|\partial^{\kappa} g_{1}\right\|_{L^{\infty}\left(U_{8}, P_{0}^{\perp}\right)} \leq C \operatorname{Lip}_{g}
$$

2. $g_{2}$ is Lipschitz continuous on $U_{8}$ with Lipschitz constant $C(\nu) \operatorname{Lip}_{g}$.

Proof. We set for $x \in P_{0}$

$$
\begin{aligned}
g_{11}(x) & :=\int_{2}^{\infty}\left(\nu_{t} * \nu_{t} * g\right)(x) \frac{\mathrm{d} t}{t} \\
g_{12}(x) & :=\int_{0}^{2}\left(\nu_{t} *\left(\mathbb{1}_{P_{0} \backslash U_{10}} \cdot\left(\nu_{t} * g\right)\right)\right)(x) \frac{\mathrm{d} t}{t}
\end{aligned}
$$

so that $g_{1}=g_{11}+g_{12}$ and we set

$$
\varphi(x):=\int_{2}^{\infty}\left(\nu_{t} * \nu_{t}\right)(x) \frac{\mathrm{d} t}{t} .
$$

At first, we prove some intermediate results:

I. $g_{12}(x)=0$ for all $x \in U_{8}$

Proof. The integrand of $g_{12}$ is

$$
\left(\nu_{t} *\left(\mathbb{1}_{P_{0} \backslash U_{10}} \cdot\left(\nu_{t} * g\right)\right)\right)(x)=\int_{P_{0}} \nu_{t}(x-y)\left(\mathbb{1}_{P_{0} \backslash U_{10}} \cdot\left(\nu_{t} * g\right)\right)(y) \mathrm{d} \mathcal{H}^{n}(y),
$$

where $x \in U_{8}$ and $0<t \leq 2$. By definition of $\nu_{t}$ and $t \leq 2$, we obtain

$$
\operatorname{supp}\left(\nu_{t}\right) \subset \operatorname{supp}\left(\nu_{2}\right) \subset B(0,2) \cap P_{0}
$$


and hence, because $x \in U_{8}$,

$$
\operatorname{supp}\left(\nu_{t}(x-\cdot)\right) \subset B(x, 2) \cap P_{0} \subset U_{10} .
$$

Furthermore, we get

$$
\operatorname{supp}\left(\mathbb{1}_{P_{0} \backslash U_{10}} \cdot\left(\nu_{t} * g\right)\right) \subset P_{0} \backslash U_{10} .
$$

Now we conclude for all $y \in P_{0}$ using $\nu \in C_{0}^{\infty}(B(0,1))$

$$
\nu_{t}(x-y)\left(\mathbb{1}_{P_{0} \backslash U_{10}} \cdot\left(\nu_{t} * g\right)\right)(y)=0,
$$

and so for all $0<t \leq 2, x \in U_{8}$

$$
\left(\nu_{t} *\left(\mathbb{1}_{P_{0} \backslash U_{10}} \cdot\left(\nu_{t} * g\right)\right)\right)(x)=0,
$$

which gives the statement.

II. For every multi-index $\kappa$, there exists some constant $C=C(n, \nu, \kappa)$ such that $\left|\partial^{\kappa} \varphi\right| \leq C$, where $\partial^{\kappa} \varphi(y):=\int_{2}^{\infty} \partial^{\kappa}\left(\nu_{t} * \nu_{t}\right)(y) \frac{\mathrm{d} t}{t}$.

Proof. We have $\operatorname{supp}(\nu(\dot{\bar{t}})) \subset B(0, t)$ and hence, for all $y \in B(0,4) \cap P_{0}$,

$$
\begin{aligned}
\left|\left(\nu_{t} * \nu_{t}\right)(y)\right| & =\left|\int_{P_{0}} \frac{1}{t^{2 n}} \nu\left(\frac{y-x}{t}\right) \nu\left(\frac{x}{t}\right) \mathrm{d} \mathcal{H}^{n}(x)\right| \\
& \leq \frac{1}{t^{2 n}} \int_{P_{0} \cap B(0, t)}\|\nu\|_{L^{\infty}\left(P_{0}, \mathbb{R}\right)}^{2} \mathrm{~d} \mathcal{H}^{n}(x) \\
& =\|\nu\|_{L^{\infty}\left(P_{0}, \mathbb{R}\right)}^{2} \frac{\omega_{n}}{t^{n}},
\end{aligned}
$$

and analogously, using $\partial^{\kappa}\left(\nu_{t}(y)\right)=\partial^{\kappa}\left(t^{-n} \nu\left(\frac{y}{t}\right)\right)=\frac{1}{t^{\kappa \kappa}}\left(\partial^{\kappa} \nu\right)_{t}(y)$, we get

$$
\left|\partial^{\kappa}\left(\nu_{t} * \nu_{t}\right)(y)\right|=\frac{1}{t^{|\kappa|}}\left|\left(\left(\partial^{\kappa} \nu\right)_{t} * \nu_{t}\right)(y)\right| \leq\|\nu\|_{L^{\infty}\left(P_{0}, \mathbb{R}\right)}\left\|\partial^{\kappa} \nu\right\|_{L^{\infty}\left(P_{0}, \mathbb{R}\right)} \frac{\omega_{n}}{t^{n+|\kappa|}} .
$$

This implies for all $y \in P_{0}$

$$
\left.\left|\partial^{\kappa} \varphi(y)\right| \leq \int_{2}^{\infty} \mid \partial^{\kappa}\left(\nu_{t} * \nu_{t}\right)(y)\right) \frac{\mathrm{d} t}{t} \leq C(n, \nu, \kappa) .
$$

III. For every multi-index $\kappa$, the function $\partial^{\kappa} \varphi$ has bounded support in $B(0,4) \cap P_{0}$.

Proof. Let $0<t \leq 2$. We have

$$
\operatorname{supp}\left(\nu_{t}\right) \subset \operatorname{supp}\left(\nu_{2}\right) \subset B(0,2)
$$

and hence

$$
\operatorname{supp}\left(\nu_{t} * \nu_{t}\right) \subset B(0,4)
$$


which implies

$$
\left(\nu_{t} * \nu_{t}\right)(x)=0
$$

for all $x \in P_{0} \backslash B(0,4)$. It follows that

$$
\int_{0}^{2}\left(\nu_{t} * \nu_{t}\right)(x) \frac{\mathrm{d} t}{t}=0
$$

for all $x \in P_{0} \backslash B(0,4)$. Now we consider $\varphi$ as a tempered distribution. The convolution with $\delta_{0}$, the Dirac mass at the origin [Gra08, Example 2.3.5, 1.], is an identity (cf. [Gra08, Example 2.3.14]), hence we get with Calderón's identity (Lemma 7.5) for all $\eta \in \mathscr{S}\left(P_{0}\right)$ with $\eta(0)=0$

$$
\begin{aligned}
\varphi(\eta)=\varphi(\eta)-\delta_{0}(\eta) & =\left(\int_{2}^{\infty}\left(\nu_{t} * \nu_{t}\right) \frac{\mathrm{d} t}{t}\right)(\eta)-\left(\int_{0}^{\infty}\left(\nu_{t} * \nu_{t} * \delta_{0}\right) \frac{\mathrm{d} t}{t}\right)(\eta) \\
& =\left(\int_{2}^{\infty}\left(\nu_{t} * \nu_{t}\right) \frac{\mathrm{d} t}{t}\right)(\eta)-\left(\int_{0}^{\infty}\left(\nu_{t} * \nu_{t}\right) \frac{\mathrm{d} t}{t}\right)(\eta) \\
& =-\left(\int_{0}^{2}\left(\nu_{t} * \nu_{t}\right) \frac{\mathrm{d} t}{t}\right)(\eta) .
\end{aligned}
$$

Since this holds for arbitrary $\eta \in \mathscr{S}\left(P_{0}\right)$ with $\operatorname{supp}(\eta) \subset P_{0} \backslash B(0,4)$, we conclude that for such $\eta$ we have

$$
\int_{P_{0}} \varphi(x) \eta(x) \mathrm{d} \mathcal{H}^{n}(x)=-\int_{P_{0}} \int_{0}^{2}\left(\nu_{t} * \nu_{t}\right)(x) \frac{\mathrm{d} t}{t} \eta(x) \mathrm{d} \mathcal{H}^{n}(x)=0
$$

and hence

$$
\operatorname{supp}(\varphi) \subset B(0,4) \cap P_{0} .
$$

For the same kind of $\eta$, we get using Fubini's theorem and partial integration

$$
\begin{aligned}
\int_{P_{0}} \partial^{\kappa} \varphi(x) \eta(x) \mathrm{d} \mathcal{H}^{n}(x) & =\int_{2}^{\infty} \int_{P_{0}} \partial^{\kappa}\left(\nu_{t} * \nu_{t}\right)(x) \eta(x) \mathrm{d} \mathcal{H}^{n}(x) \frac{\mathrm{d} t}{t} \\
& =(-1)^{|\kappa|} \int_{2}^{\infty} \int_{P_{0}}\left(\nu_{t} * \nu_{t}\right)(x) \partial^{\kappa} \eta(x) \mathrm{d} \mathcal{H}^{n}(x) \frac{\mathrm{d} t}{t} \\
& =(-1)^{|\kappa|} \int_{P_{0}} \varphi(x) \partial^{\kappa} \eta(x) \mathrm{d} \mathcal{H}^{n}(x) \\
& =0
\end{aligned}
$$

since $\partial^{\kappa} \eta \in \mathscr{S}\left(P_{0}\right)$ with $\operatorname{supp}\left(\partial^{\kappa} \eta\right) \subset P_{0} \backslash B(0,4)$.

IV. $\varphi \in C_{0}^{\infty}\left(P_{0}\right)$

Proof. With II. and III. we conclude for every multi-index $\kappa$ that $\partial^{\kappa} \varphi \in L^{1}\left(P_{0}, \mathbb{R}\right)$. With Fubini's theorem and partial integration, we see that $\partial^{\kappa} \varphi$ is the weak derivative of $\varphi$ hence we have $\varphi \in W^{l, 1}\left(P_{0}\right)$ for every $l \in \mathbb{N}$. The Sobolev imbedding theorem [AF03, Thm 4.12] gives us $\varphi \in C^{\infty}\left(P_{0}\right)$ and, with II., we obtain $\varphi \in C_{0}^{\infty}\left(P_{0}\right)$. 
Now we have for all $x \in U_{8}$ with Fubini's theorem [EG92, 1.4, Thm. 1]

$$
\begin{aligned}
g_{11}(x) & =\int_{2}^{\infty}\left(\nu_{t} * \nu_{t} * g\right)(x) \frac{\mathrm{d} t}{t} \\
& =\int_{2}^{\infty} \int_{P_{0}}\left(\nu_{t} * \nu_{t}\right)(x-y) g(y) \mathrm{d} \mathcal{H}^{n}(y) \frac{\mathrm{d} t}{t} \\
& =\int_{P_{0}} \int_{2}^{\infty}\left(\nu_{t} * \nu_{t}\right)(x-y) \frac{\mathrm{d} t}{t} g(y) \mathrm{d} \mathcal{H}^{n}(y) \\
& =\int_{P_{0}} \varphi(x-y) g(y) \mathrm{d} \mathcal{H}^{n}(y) \\
& =(\varphi * g)(x) .
\end{aligned}
$$

We know, that $\varphi \in C_{0}^{\infty}\left(P_{0}\right)$ and $g \in C_{0}^{0,1}\left(P_{0}, P_{0}^{\perp}\right)$. Hence we have $g_{11} \in C_{0}^{\infty}\left(P_{0}\right), g \in$ $W^{1, \infty}\left(P_{0}\right)$ and for $i, j \in\{1, \ldots, n\}$

$$
\begin{aligned}
\partial_{i} g_{11} & =\varphi * \partial_{i} g, \\
\partial_{i} \partial_{j} g_{11} & =\partial_{i} \varphi * \partial_{j} g .
\end{aligned}
$$

With the Minkowski inequality [Gra08, Thm. 1.2.10] and III., we obtain for $i, j \in\{1, \ldots, n\}$

$$
\begin{aligned}
\left\|\partial_{i} g_{1}\right\|_{L^{\infty}\left(U_{8}, \mathbb{R}\right)} \stackrel{\text { I. }}{=}\left\|\partial_{i} g_{11}\right\|_{L^{\infty}\left(U_{8}, \mathbb{R}\right)} \leq\left\|\partial_{i} g\right\|_{L^{\infty}\left(U_{8}, \mathbb{R}\right)}\|\varphi\|_{L^{1}\left(P_{0}\right)} \leq C(\nu) \operatorname{Lip}_{g}, \\
\left\|\partial_{i} \partial_{j} g_{1}\right\|_{L^{\infty}\left(U_{8}, \mathbb{R}\right)} \stackrel{\text { I. }}{=}\left\|\partial_{i} \partial_{j} g_{11}\right\|_{L^{\infty}\left(U_{8}, \mathbb{R}\right)} \leq\left\|\partial_{i} g\right\|_{L^{\infty}\left(U_{8}, \mathbb{R}\right)}\left\|\partial_{j} \varphi\right\|_{L^{1}\left(P_{0}\right)} \leq C(\nu) \operatorname{Lip}_{g} .
\end{aligned}
$$

Now it is easy to see that $g_{2}$ is $C \operatorname{Lip}_{g}$-Lipschitz on $U_{8}$ because we have $g_{2}=g-g_{1}$ and $g$ as well as $g_{1}$ are $C \operatorname{Lip}_{g}$-Lipschitz on $U_{8}$.

Remark 7.7. Under the assumption that

$$
\int_{U_{10}}\left(\int_{0}^{2} \gamma_{g}(x, t)^{2} \frac{\mathrm{d} t}{t}\right)^{\frac{p}{2}} \mathrm{~d} \mathcal{H}^{n}(x)<\infty,
$$

the next lemmas will prove that $g_{2} \in W_{0}^{1, p}\left(P_{0}, P_{0}^{\perp}\right)$. We show for this purpose in Lemma 7.10 that

$$
\partial_{i} g_{2}(x):=\int_{0}^{2} \partial_{i}\left(\nu_{t} *\left(\mathbb{1}_{U_{10}}\left(\nu_{t} * g\right)\right)\right)(x) \frac{\mathrm{d} t}{t}
$$

is in $L^{p}\left(P_{0}, P_{0}^{\perp}\right)$. In fact $\partial_{i} g_{2}$ is the weak derivative of $g_{2}$ because for all $\varrho \in C_{0}^{\infty}\left(P_{0}, \mathbb{R}\right)$ we get with Fubini's theorem [EG92, 1.4, Thm. 1] and partial integration for $i \in\{1, \ldots, n\}$

$$
\begin{aligned}
\int_{P_{0}} \partial_{i} g_{2}(x) \varrho(x) \mathrm{d} \mathcal{H}^{n}(x) & =\int_{P_{0}} \int_{0}^{2} \partial_{i}\left(\nu_{t} *\left(\mathbb{1}_{U_{10}}\left(\nu_{t} * g\right)\right)\right)(x) \frac{\mathrm{d} t}{t} \varrho(x) \mathrm{d} \mathcal{H}^{n}(x) \\
& =\int_{0}^{2} \int_{P_{0}} \partial_{i}\left(\nu_{t} *\left(\mathbb{1}_{U_{10}}\left(\nu_{t} * g\right)\right)\right)(x) \varrho(x) \mathrm{d} \mathcal{H}^{n}(x) \frac{\mathrm{d} t}{t} \\
& =-\int_{0}^{2} \int_{P_{0}}\left(\nu_{t} *\left(\mathbb{1}_{U_{10}}\left(\nu_{t} * g\right)\right)\right)(x) \partial_{i} \varrho(x) \mathrm{d} \mathcal{H}^{n}(x) \frac{\mathrm{d} t}{t} \\
& =-\int_{P_{0}} g_{2}(x) \partial_{i} \varrho(x) \mathrm{d} \mathcal{H}^{n}(x) .
\end{aligned}
$$


Lemma 7.8. We have for $i \in\{1, \ldots, n\}$

$$
\begin{aligned}
& \operatorname{supp}\left(g_{2}\right) \subset B(0,12) \cap P_{0}, \\
& \operatorname{supp}\left(\partial_{i} g_{2}\right) \subset B(0,12) \cap P_{0} .
\end{aligned}
$$

Proof. If $0<t<2$ and $x \in P_{0}$, we have $\operatorname{supp}\left(\nu_{t}(x-\cdot)\right) \subset B(x, 2) \cap P_{0}$ and $\operatorname{supp}\left(\mathbb{1}_{U_{10}}\left(\nu_{t} * g\right)\right) \subset$ $B(0,10) \cap P_{0}$. This implies $\operatorname{supp}\left(\nu_{t} *\left(\mathbb{1}_{U_{10}}\left(\nu_{t} * g\right)\right)\right) \subset B(0,12) \cap P_{0}$ and hence we obtain $\operatorname{supp}\left(g_{2}\right) \subset B(0,12)$ and $\operatorname{supp}\left(\partial_{i} g_{2}\right) \subset B(0,12) \cap P_{0}$.

Lemma 7.9. Let $x \in U_{10}$ and $0<t<2$. We have

$$
\left|\frac{\left(\nu_{t} * g\right)(x)}{t}\right| \leq\|\nu\|_{L^{\infty}\left(P_{0}, \mathbb{R}\right)} \gamma_{g}(x, t) .
$$

Proof. If $a: P_{0} \rightarrow P_{0}^{\perp}$ is an affine function, we get

$$
\left(\nu_{t} * a\right)(x)=\int_{P_{0}} \frac{1}{t^{n}} \nu\left(\frac{x-y}{t}\right) a(y) \mathrm{d} \mathcal{H}^{n}(y)=(-1)^{n} \int_{P_{0}} \nu(z) \underbrace{a(x-t z)}_{\text {affine }} \mathrm{d} \mathcal{H}^{n}(z) \stackrel{(7.5)}{=} 0
$$

and hence, with Lemma 7.41.

$$
\begin{aligned}
\left|\frac{\left(\nu_{t} * g\right)(x)}{t}\right| & =\left|\frac{\left(\nu_{t} * g\right)(x)-\left(\nu_{t} * a\right)(x)}{t}\right| \\
& =\left|\frac{\left(\nu_{t} *(g-a)\right)(x)}{t}\right| \\
& =\left|\int_{P_{0}} \frac{1}{t} \nu_{t}(x-y)(g(y)-a(y)) \mathrm{d} \mathcal{H}^{n}(y)\right| \\
& \leq \frac{1}{t^{n}} \int_{P_{0}}\left|\frac{\nu\left(\frac{x-y}{t}\right)(g(y)-a(y))}{t}\right| \mathrm{d} \mathcal{H}^{n}(y) \\
& \leq\|\nu\|_{L^{\infty}\left(P_{0}, \mathbb{R}\right)} \frac{1}{t^{n}} \int_{P_{0} \cap B(x, t)}\left|\frac{g(y)-a(y)}{t}\right| \mathrm{d} \mathcal{H}^{n}(y) .
\end{aligned}
$$

Since $a$ was an arbitrary affine function, we conclude

$$
\begin{aligned}
\left|\frac{\left(\nu_{t} * g\right)(x)}{t}\right| & \leq\|\nu\|_{L^{\infty}\left(P_{0}, \mathbb{R}\right)} \inf _{a \in \mathcal{A}\left(P_{0}, P_{0}^{\perp}\right)} \frac{1}{t^{n}} \int_{B(x, t) \cap P_{0}} \frac{d(g(z), a(z))}{t} \mathrm{~d} \mathcal{H}^{n}(z) \\
& =\|\nu\|_{L^{\infty}\left(P_{0}, \mathbb{R}\right)} \gamma_{g}(x, t) .
\end{aligned}
$$

We have $p \in(1, \infty)$ and, for the proof of Theorem 7.3 , we can assume that

$$
\int_{U_{10}}\left(\int_{0}^{2} \gamma_{g}(x, t)^{2} \frac{\mathrm{d} t}{t}\right)^{\frac{p}{2}} \mathrm{~d} \mathcal{H}^{n}(x)<\infty
$$


Lemma 7.10. We have $g_{2} \in W_{0}^{1, p}\left(P_{0}, P_{0}^{\perp}\right)$ and there exists some constant $C=C(n, p, \nu)$, so that for all $i \in\{1, \ldots, n\}$

$$
\left\|\partial_{i} g_{2}\right\|_{L^{p}\left(P_{0}, P_{0}^{\perp}\right)}^{p} \leq C \int_{U_{10}}\left(\int_{0}^{2} \gamma_{g}(x, t)^{2} \frac{\mathrm{d} t}{t}\right)^{\frac{p}{2}} \mathrm{~d} \mathcal{H}^{n}(x),
$$

where $\partial_{i} g_{2}(x)=\int_{0}^{2} \partial_{i}\left(\nu_{t} *\left(\mathbb{1}_{U_{10}}\left(\nu_{t} * g\right)\right)\right)(x) \frac{\mathrm{d} t}{t}$.

Proof. We recall that $\partial_{i} g_{2}$ is the weak derivative of $g_{2}$ (cf. Remark 7.7). Due to [AF03, Cor 6.31, An Equivalent Norm for $\left.W_{0}^{m, p}(\Omega)\right]$ and Lemma 7.8, we only have to consider $\left\|\partial_{i} g_{2}\right\|_{L^{p}\left(P_{0}\right)}$ for all $i \in\{0, \ldots, n\}$ to get $g_{2} \in W_{0}^{1, p}\left(P_{0}, P_{0}^{\perp}\right)$.

For $x \in P_{0}$, we have $\partial_{i} \nu_{t}(x)=\partial_{i} t^{-n} \nu\left(\frac{x}{t}\right)=t^{-1}\left(\partial_{i} \nu\right)_{t}(x)$ and hence

$$
\partial_{i} g_{2}(x)=\int_{0}^{2} \partial_{i}\left(\nu_{t} *\left(\mathbb{1}_{U_{10}}\left(\nu_{t} * g\right)\right)\right)(x) \frac{\mathrm{d} t}{t}=\int_{0}^{2}\left(\left(\partial_{i} \nu\right)_{t} *\left(\mathbb{1}_{U_{10}}\left(\frac{\nu_{t} * g}{t}\right)\right)\right)(x) \frac{\mathrm{d} t}{t} .
$$

We want to show that the $L^{p}$ norm of $\partial_{i} g_{2}$ is bounded. Via duality, we obtain (cf. [AF03, The Normed Dual of $\left.L^{p}(\Omega)\right]$ )

$$
\left\|\partial_{i} g_{2}\right\|_{L^{p}\left(P_{0}\right)}=\sup _{\|f\|_{L^{p^{\prime}\left(P_{0}\right)}}=1}\left|\int_{P_{0}} f(x) \partial_{i} g_{2}(x) \mathrm{d} \mathcal{H}^{n}(x)\right|
$$

where $\frac{1}{p}+\frac{1}{p^{\prime}}=1$. So, let $f \in L^{p^{\prime}}\left(P_{0}\right)$ with $\|f\|_{L^{p^{\prime}}\left(P_{0}\right)}=1$. We get with property (7.6) from Lemma 7.4, Fubini's theorem [EG92, 1.4, Thm. 1] and Hölder's inequality

$$
\begin{aligned}
& \left|\int_{P_{0}} f(x) \partial_{i} g_{2}(x) \mathrm{d} \mathcal{H}^{n}(x)\right| \\
= & \left|\int_{P_{0}} f(x) \int_{0}^{2} \int_{P_{0}}\left(\partial_{i} \nu\right)_{t}(x-y)\left(\mathbb{1}_{U_{10}}\left(\frac{\nu_{t} * g}{t}\right)\right)(y) \mathrm{d} \mathcal{H}^{n}(y) \frac{\mathrm{d} t}{t} \mathrm{~d} \mathcal{H}^{n}(x)\right| \\
\stackrel{(7.6)}{=} & \left|\int_{P_{0}} \int_{0}^{2} \int_{P_{0}}-\left(\partial_{i} \nu\right)_{t}(y-x) f(x) \mathrm{d} \mathcal{H}^{n}(x)\left(\mathbb{1}_{U_{10}}\left(\frac{\nu_{t} * g}{t}\right)\right)(y) \frac{\mathrm{d} t}{t} \mathrm{~d} \mathcal{H}^{n}(y)\right| \\
\leq & \int_{P_{0}} \int_{0}^{2}\left|\left(\left(\partial_{i} \nu\right)_{t} * f\right)(y)\right|\left|\left(\mathbb{1}_{U_{10}}\left(\frac{\nu_{t} * g}{t}\right)\right)(y)\right| \frac{\mathrm{d} t}{t} \mathrm{~d} \mathcal{H}^{n}(y) \\
\leq & \int_{P_{0}}\left(\int_{0}^{2}\left|\left(\left(\partial_{i} \nu\right)_{t} * f\right)(y)\right|^{2} \frac{\mathrm{d} t}{t}\right)^{\frac{1}{2}}\left(\int_{0}^{2}\left|\left(\mathbb{1}_{U_{10}}\left(\frac{\nu_{t} * g}{t}\right)\right)(y)\right|^{2} \frac{\mathrm{d} t}{t}\right)^{\frac{1}{2}} \mathrm{~d} \mathcal{H}^{n}(y) \\
\leq & ||\left(\int_{0}^{\infty}\left|\left(\partial_{i} \nu\right)_{t} * f\right|^{2} \frac{\mathrm{d} t}{t}\right)^{\frac{1}{2}}||_{L^{p^{\prime}}\left(P_{0}\right)} \\
& \left(\int_{P_{0}}\left(\int_{0}^{2}\left|\left(\mathbb{1}_{U_{10}}\left(\frac{\nu_{t} * g}{t}\right)\right)(y)\right|^{2} \frac{\mathrm{d} t}{t}\right)^{\frac{p}{2}} \mathrm{~d} \mathcal{H}^{n}(y)\right)^{\frac{1}{p}} .
\end{aligned}
$$

There exists some constant $C=C(n, \nu)$ with $\left|\partial_{i} \nu(x)\right|+\left|\nabla \partial_{i} \nu(x)\right| \leq C(1+|x|)^{-n-1}$ because $\nu$ is a Schwartz function. Together with Lemma 7.4, all the requirements of Lemma A.23 with 
$\phi=\partial_{i} \nu$ and $q=p^{\prime}$ are fulfilled, which implies, since $\|f\|_{L^{p}\left(P_{0}\right)}=1$, that the first factor in (7.8) is some constant $C(n, p, \nu)$ independent of $f$. All in all, we obtain

$$
\begin{aligned}
\left\|\partial_{i} g_{2}\right\|_{L^{p}\left(P_{0}\right)} & =\sup _{\|f\|_{L^{p^{\prime}\left(P_{0}\right)}}=1}\left|\int_{P_{0}} f(x) \partial_{i} g_{2}(x) \mathrm{d} \mathcal{H}^{n}(x)\right| \\
& \stackrel{(7.8)}{\leq} C(n, p, \nu)\left(\int_{P_{0}}\left(\int_{0}^{2}\left|\left(\mathbb{1}_{U_{10}}\left(\frac{\nu_{t} * g}{t}\right)\right)(y)\right|^{2} \frac{\mathrm{d} t}{t}\right)^{\frac{p}{2}} \mathrm{~d} \mathcal{H}^{n}(y)\right)^{\frac{1}{p}} .
\end{aligned}
$$

Finaly, we deduce with Lemma 7.9

$$
\int_{P_{0}}\left\|\partial_{i} g_{2}\right\|^{p}(x) \mathrm{d}(x) \leq C(N, p, \nu) \int_{P_{0} \cap U_{10}}\left(\int_{0}^{2} \gamma_{g}(x, t)^{2} \frac{\mathrm{d} t}{t}\right)^{\frac{p}{2}} \mathrm{~d} \mathcal{H}^{n}(x) .
$$

Definition 7.11. Let $B$ be a ball with centre in $P_{0}$ and $f: P_{0} \rightarrow P_{0}^{\perp}$ be some map. We define the average of $f$ on $B$

$$
\underset{B}{\operatorname{Avg}(f)}:=\frac{1}{(\operatorname{diam} B)^{n}} \int_{B \cap P_{0}} f \mathrm{~d} \mathcal{H}^{n},
$$

some maximal function

$$
N(f)(x):=\sup _{\substack{t \in(0, \infty), y \in P_{0} \\ \text { with } d(y, x) \leq t}}\left\{\frac{1}{2 t} \operatorname{Avg}_{B(y, t)}(\mid f-\underset{B(y, t)}{\operatorname{Avg}(f) \mid)\}}\right.
$$

where $x \in P_{0}$, and the oscillation of $f$ on $B$

$$
\operatorname{osc}_{B}(f):=\sup _{x \in B \cap P_{0}}\left|f(x)-\operatorname{Avg}_{B}(f)\right| .
$$

Lemma 7.12. There exists some constant $C=C(n)$ so that

$$
\left\|N\left(g_{2}\right)\right\|_{L^{p}\left(P_{0}, \mathbb{R}\right)} \leq C \frac{p 3^{\frac{n}{p}}}{p-1}\left\|D g_{2}\right\|_{L^{p}\left(P_{0}, P_{0}^{\perp}\right)} .
$$

Proof. With the Poincaré inequality [EG92, 4.5.2, Thm. 2, first part of the proof], there exists some constant $C(n)$ so that $\left(g_{2} \in W_{0}^{1, p}\left(P_{0}, P_{0}^{\perp}\right)\right.$, see Lemma 7.9)

$$
\begin{aligned}
& \underset{B}{\operatorname{Avg}}\left(\left|g_{2}-\underset{B}{\operatorname{Avg}}\left(g_{2}\right)\right|\right)=\frac{1}{(\operatorname{diam} B)^{n}} \int_{B \cap P_{0}} \mid g_{2}(x)-\underset{B}{\operatorname{Avg}\left(g_{2}\right) \mid \mathrm{d} \mathcal{H}^{n}(x)} \\
& \leq C(n) \operatorname{diam} B \frac{1}{(\operatorname{diam} B)^{n}} \int_{B \cap P_{0}}\left|D g_{2}(y)\right| \mathrm{d} \mathcal{H}^{n}(y) \\
& =C(n) \operatorname{diam} B \underset{B}{\operatorname{Avg}\left(\left|D g_{2}\right|\right),}
\end{aligned}
$$


(if $f$ is a Matrix, we denote by $|f|$ a matrix norm) and hence we get for $x \in P_{0}$

$$
\begin{aligned}
N\left(g_{2}\right)(x) & =\sup _{\substack{t \in(0, \infty), y \in P_{0} \\
\text { with } d(y, x) \leq t}}\left\{\frac { 1 } { 2 t } \operatorname { A v g } _ { B ( y , t ) } \left(\mid g_{2}-\underset{B(y, t)}{\left.\left.\operatorname{Avg}\left(g_{2}\right) \mid\right)\right\}}\right.\right. \\
& \leq C(n) \sup _{\substack{t \in(0, \infty), y \in P_{0} \\
\text { with } d(y, x) \leq t}} \operatorname{Avg}\left(\left|D g_{2}\right|\right) \\
& =C(n) M\left(D g_{2}\right)(x),
\end{aligned}
$$

where $M\left(D g_{2}\right)$ is the uncentred Hardy-Littlewood maximal function. Finally, we obtain with [Gra08, Thm. 2.1.6]

$$
\left\|N\left(g_{2}\right)\right\|_{L^{p}\left(P_{0}, \mathbb{R}\right)} \leq C(n)\left\|M\left(D g_{2}\right)\right\|_{L^{p}\left(P_{0}, \mathbb{R}\right)} \leq C(n) \frac{p 3^{\frac{n}{p}}}{p-1}\left\|D g_{2}\right\|_{L^{p}\left(P_{0}, P_{0}^{\perp}\right)} .
$$

Definition 7.13. Let $\theta>0$. We define

$$
H_{\theta}:=\left\{x \in U_{6} \mid N\left(g_{2}\right)(x) \leq \theta^{n+1} \operatorname{Lip}_{g}\right\} .
$$

Lemma 7.14. Let $\theta>0$. There exists some constant $C=C(n, p, \nu)$ so that

$$
\mathcal{H}^{n}\left(U_{6} \backslash H_{\theta}\right) \leq \frac{C}{\theta^{p(n+1)} \operatorname{Lip}_{g}^{p}} \int_{U_{10}}\left(\int_{0}^{2} \gamma_{g}(x, t)^{2} \frac{\mathrm{d} t}{t}\right)^{\frac{p}{2}} \mathrm{~d} \mathcal{H}^{n}(x) .
$$

Proof. With Lemma 7.12, Lemma 7.10 and $\left\|D g_{2}\right\|_{L^{p}\left(P_{0}, P_{0}^{\perp}\right)}^{p} \leq n^{p-1} \sum_{i=1}^{n}\left\|\partial_{i} g_{2}\right\|_{L^{p}\left(P_{0}, P_{0}^{\perp}\right)}^{p}$, there exists some constant $C=C(n, p, \nu)$ with

$$
\left\|N\left(g_{2}\right)\right\|_{L^{p}\left(P_{0}, \mathbb{R}\right)}^{p} \leq \frac{3^{n}}{n}\left(\frac{C n p}{p-1}\right)^{p} \sum_{i=1}^{n}\left\|\partial_{i} g_{2}\right\|_{L^{p}\left(P_{0}, P_{0}^{\perp}\right)}^{p} \leq C \int_{U_{10}}\left(\int_{0}^{2} \gamma_{g}(x, t)^{2} \frac{\mathrm{d} t}{t}\right)^{\frac{p}{2}} \mathrm{~d} \mathcal{H}^{n}(x) .
$$

Hence we get

$$
\begin{aligned}
\mathcal{H}^{n}\left(U_{6} \backslash H_{\theta}\right) & =\frac{1}{\theta^{p(n+1)} \operatorname{Lip}_{g}^{p}} \int_{U_{6} \backslash H_{\theta}} \theta^{p(n+1)} \operatorname{Lip}_{g}^{p} \mathrm{~d} \mathcal{H}^{n}(y) \\
& <\frac{1}{\theta^{p(n+1)} \operatorname{Lip}_{g}^{p}} \int_{U_{6} \backslash H_{\theta}} N\left(g_{2}\right)^{p}(y) \mathrm{d} \mathcal{H}^{n}(y) \\
& \leq \frac{C}{\theta^{p(n+1)} \operatorname{Lip}_{g}^{p}} \int_{U_{10}}\left(\int_{0}^{2} \gamma_{g}(x, t)^{2} \frac{\mathrm{d} t}{t}\right)^{\frac{p}{2}} \mathrm{~d} \mathcal{H}^{n}(x) .
\end{aligned}
$$

Lemma 7.15. Let $B$ be a ball with centre in $P_{0}$. If $\left(B \cap P_{0}\right) \subset U_{8}$, then there exists some constant $C=C(N, n, \nu)$ with

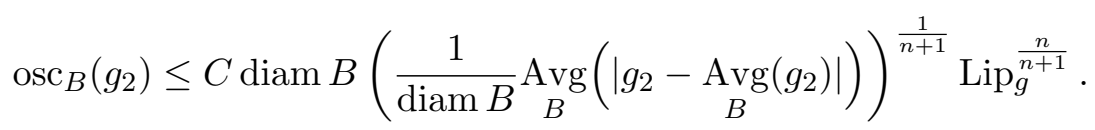


Proof. Let $\left(B \cap P_{0}\right) \subset U_{8}$ and $\lambda:=\operatorname{osc}_{B}\left(g_{2}\right)$. The function $g_{2}$ is Lipschitz continuous on $U_{8}$ with $\operatorname{Lip}_{g_{2}}=C(\nu) \operatorname{Lip}_{g}$ (see Lemma 7.6 on page 93) and $B \cap P_{0}$ is closed. Hence there exists some $y \in B \cap P_{0}$ with $\lambda=\left|g_{2}(y)-\operatorname{Avg}_{B} g_{2}\right|$ and we get for $x \in B$ with $d(x, y) \leq \frac{\lambda}{2 \operatorname{Lip}_{g_{2}}}$

$$
\left|g_{2}(x)-\underset{B}{\operatorname{Avg}}\left(g_{2}\right)\right| \geq\left|g_{2}(y)-\underset{B}{\operatorname{Avg}}\left(g_{2}\right)\right|-\left|g_{2}(x)-g_{2}(y)\right| \geq \lambda-\operatorname{Lip}_{g_{2}} d(x, y) \geq \frac{\lambda}{2} .
$$

Furthermore, using that $g_{2}$ is continuous on $U_{8}$ for all $l \in\{1, \ldots, N\}$, there exists some $z_{l} \in B \cap P_{0}$, with $g_{2}^{l}\left(z_{l}\right)=\underset{B}{\operatorname{Avg}}\left(g_{2}^{l}\right)$ (where $g_{2}^{l}\left(z_{l}\right) \in \mathbb{R}$ means the $l$-th component of $g_{2}\left(z_{l}\right) \in \mathbb{R}^{N}$ ), and so

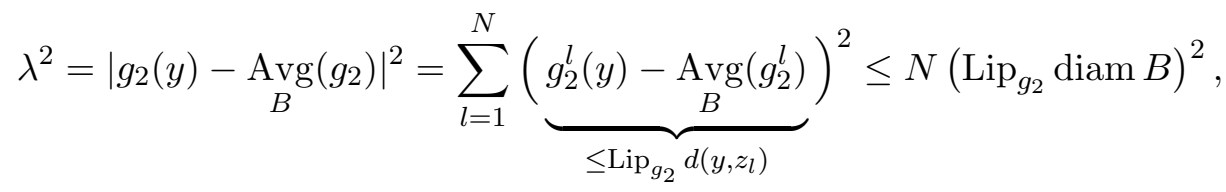

which implies $\frac{\lambda}{\sqrt{N} \operatorname{Lip}_{g_{2}}} \leq \operatorname{diam} B$. Since $y \in B$, there exists some ball $\hat{B} \subset B \cap B\left(y, \frac{\lambda}{2 \operatorname{Lip}_{g_{2}}}\right)$ with

$$
\operatorname{diam} \hat{B}=\min \left(\frac{\lambda}{2 \operatorname{Lip}_{g_{2}}}, \frac{\operatorname{diam} B}{2}\right) \geq \frac{\lambda}{2 \sqrt{N} \operatorname{Lip}_{g_{2}}}
$$

and hence

$$
\begin{aligned}
\int_{B \cap P_{0}} \mid g_{2}(x)-\underset{B}{\operatorname{Avg}\left(g_{2}\right) \mid \mathrm{d} \mathcal{H}^{n}(x)} & \geq \int_{\hat{B} \cap P_{0}} \mid g_{2}(x)-\underset{B}{\operatorname{Avg}\left(g_{2}\right) \mid \mathrm{d} \mathcal{H}^{n}(x)} \\
& \geq \omega_{n}\left(\frac{\lambda}{4 \sqrt{N} \operatorname{Lip}_{g_{2}}}\right)^{n} \frac{\lambda}{2}
\end{aligned}
$$

This implies with $\operatorname{Lip}_{g_{2}}=C(\nu) \operatorname{Lip}_{g}$

$$
\lambda^{n+1} \leq C(N, n, \nu) \operatorname{Lip}_{g}^{n}(\operatorname{diam} B)^{n+1} \frac{\operatorname{Avg}_{B}\left(\mid g_{2}-\underset{B}{\left.\operatorname{Avg} g_{2} \mid\right)}\right.}{\operatorname{diam} B} .
$$

Lemma 7.16. Let $y \in P_{0}$. There exists some constant $C=C(N, n, \nu)$ and some affine map $a_{y}: P_{0} \rightarrow P_{0}^{\perp}$ so that if $r \leq \theta$ and $B(y, r) \cap H_{\theta} \neq \emptyset$, we have

$$
\left\|g-a_{y}\right\|_{L^{\infty}\left(B(y, r) \cap P_{0}, P_{0}^{\perp}\right)} \leq C r \theta \operatorname{Lip}_{g} .
$$

Proof. Let $y \in P_{0}$. If $\theta \geq 1$, we can choose $a_{y}\left(y^{\prime}\right):=g(y)$ as a constant and get the desired result directly from the Lipschitz condition. Now let $0<\theta<1$. We set $a_{y}\left(y^{\prime}\right):=g(y)+$ $D g_{1}(y) \phi^{-1}\left(y^{\prime}-y\right)$. For some arbitrary $y^{\prime} \in B(y, r) \cap P_{0}$, we obtain (the explanations I - V 
are stated below the calculation)

$$
\begin{aligned}
& \left|g\left(y^{\prime}\right)-\left[g(y)+D g_{1}(y) \phi^{-1}\left(y^{\prime}-y\right)\right]\right| \\
& \stackrel{\mathrm{I}}{=}\left|g_{1}\left(y^{\prime}\right)+g_{2}\left(y^{\prime}\right)-\left[g_{1}(y)+g_{2}(y)+D g_{1}(y) \phi^{-1}\left(y^{\prime}-y\right)\right]\right| \\
& \leq\left|g_{2}\left(y^{\prime}\right)-g_{2}(y)\right|+\left|g_{1}\left(y^{\prime}\right)-\left[g_{1}(y)+D g_{1}(y) \phi^{-1}\left(y^{\prime}-y\right)\right]\right| \\
& \stackrel{\mathrm{II}}{\leq}\left|g_{2}\left(y^{\prime}\right)-\underset{B(y, r)}{\operatorname{Avg}}\left(g_{2}\right)\right|+\left|\underset{B(y, r)}{\operatorname{Avg}}\left(g_{2}\right)-g_{2}(y)\right|+\sum_{|\kappa|=2}\left\|\partial^{\kappa} g_{1}\right\|_{L^{\infty}\left(U_{8}\right)}\left|y^{\prime}-y\right|^{2}
\end{aligned}
$$

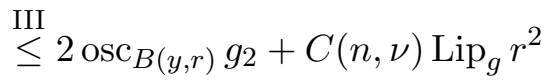

$$
\begin{aligned}
& \stackrel{\mathrm{IV}}{\leq} C(N, n, \nu) \operatorname{diam} B(y, r)\left(\frac{1}{\operatorname{diam} B(y, r)} \underset{B(y, r)}{\operatorname{Avg}}\left(\mid g_{2}-\underset{B(y, r)}{\left.\operatorname{Avg}\left(g_{2}\right) \mid\right)}\right)^{\frac{1}{n+1}} \operatorname{Lip}_{g}^{\frac{n}{n+1}}+C(n, \nu) \operatorname{Lip}_{g} r^{2}\right. \\
& \leq C(N, n, \nu) r\left(N\left(g_{2}\right)(y)\right)^{\frac{1}{n+1}} \operatorname{Lip}_{g}^{\frac{n}{n+1}}+C(n, \nu) \operatorname{Lip}_{g} r^{2} \\
& \stackrel{\mathrm{V}}{\leq} C(N, n, \nu) r \theta \operatorname{Lip}_{g} \text {. }
\end{aligned}
$$

I. We have $g=g_{1}+g_{2}$.

II. Let $y^{\prime} \in B(y, r) \cap P_{0}$. We have $d\left(y^{\prime}, U_{6}\right) \leq d\left(y^{\prime}, H_{\theta}\right) \leq d\left(y^{\prime}, y\right)+d\left(y, H_{\theta}\right) \leq 2 r \leq 2 \theta \leq 2$. So we get $y^{\prime}, y \in U_{8}$. With Lemma 7.6, we have $g_{1} \in C^{\infty}\left(U_{8}\right)$ and so we obtain with Taylor's theorem (see Lemma A.15)

$$
\begin{aligned}
& g_{1}\left(y^{\prime}\right) \\
= & g_{1}(y)+D g_{1}(y) \phi^{-1}\left(y^{\prime}-y\right)+\int_{0}^{1} 2(1-t) \sum_{|\kappa|=2} \frac{1}{\kappa !} \partial^{\kappa} g_{1}\left(y+t\left(y^{\prime}-y\right)\right)\left[\phi^{-1}\left(y^{\prime}-y\right)\right]^{\kappa} \mathrm{d} t .
\end{aligned}
$$

III. See Definition 7.11 and Lemma 7.6.

IV. Since $r \leq \theta<1, B(y, r) \cap H_{\theta} \neq \emptyset$ and $H_{\theta} \subset U_{6}$, we obtain $B(y, r) \cap P_{0} \subset U_{8}$ and we can apply Lemma 7.15.

V. See definition of $H_{\theta}$ and consider $r \leq \theta$.

Lemma 7.14 and Lemma 7.16 complete the proof of Theorem 7.3.

\subsection{The $\gamma$-function of $A$ and integral Menger curvature}

In this section, we prove the following Theorem 7.17. It states that we get a similar control on the $\gamma$-functions applied to our function $A$ as we get in Corollary 5.8 on the $\beta$-numbers.

For $\alpha, \varepsilon>0, \eta \leq 2 \varepsilon$ and $k \geq 4$, we defined $A$ on $U_{12}$ (cf. Definition 6.21 on page 78). Since in this section we only apply the $\gamma$-functions to $A$, we set $\gamma(q, t):=\gamma_{A}(q, t)$ and $\tilde{\gamma}(q, t):=\tilde{\gamma}_{A}(q, t)$. 
Theorem 7.17. There exists some $\tilde{k} \geq 4$ and some $\tilde{\alpha}=\tilde{\alpha}(n)>0$ so that, for all $\alpha$ with $0<\alpha \leq \tilde{\alpha}$, there exists some $\tilde{\varepsilon}=\tilde{\varepsilon}\left(N, n, C_{0}, \alpha\right)$ so that, if $k \geq \tilde{k}$ and $\eta \leq \tilde{\varepsilon}^{p}$, we have for all $\varepsilon \in\left[\eta^{\frac{1}{p}}, \tilde{\varepsilon}\right]$ that there exists some constant $C=C\left(N, n, \mathcal{K}, p, C_{0}, k\right)$ so that

$$
\begin{aligned}
\int_{U_{10}} \int_{0}^{2} \gamma(q, t)^{p} \frac{\mathrm{d} t}{t} \mathrm{~d} \mathcal{H}^{n}(q) & \leq C \varepsilon^{p}+C \mathcal{M}_{\mathcal{K}^{p}}(\mu) \\
& \leq C \varepsilon^{p},
\end{aligned}
$$

where $U_{10}=B(0,10) \cap P_{0}$.

Proof. Let $\bar{k} \geq 4$ be the maximum of all thresholds for $k$ given in chapter 6 and let $\tilde{\alpha}=$ $\tilde{\alpha}(n) \leq \frac{1}{4}$ be the upper bound for the Lipschitz constant given by Lemma 7.2. We set $\tilde{k}:=\max \{\bar{k}, \tilde{C}+1, \hat{C}\}$ where the constants $\tilde{C}$ and $\hat{C}$ are fixed constants which will be set during this section ${ }^{2}$. Let $0 \leq \alpha \leq \tilde{\alpha}$. Let $\bar{\varepsilon}=\varepsilon\left(N, n, C_{0}, \alpha\right) \leq \alpha$ be the minimum of all thresholds for $\varepsilon$ given in chapter 6 . We set $\tilde{\varepsilon}:=\min \left\{\bar{\varepsilon},\left(2 C^{\prime} C_{1}\right)^{-1}\right\}<1^{3}$ and assume that $k \geq \tilde{k}$ and $\eta \leq \tilde{\varepsilon}^{p}$. Now let $\varepsilon>0$ with $\eta \leq \varepsilon^{p} \leq \tilde{\varepsilon}^{p}$. For the rest of this section, we fix the parameters $k, \eta, \alpha, \varepsilon$ and mention that they meet all requirements of the lemmas in Chapter 6.

With Lemma 6.14, we obtain

$$
U_{10} \subset \bigcup_{i \in I}\left(R_{i} \cap U_{10}\right) \cup \pi(Z)=\bigcup_{i \in I_{12}}\left(R_{i} \cap U_{10}\right) \cup \pi(Z),
$$

where $I_{12}=\left\{i \in I \mid R_{i} \cap U_{12} \neq \emptyset\right\}$ (see page 76 ) and so we get

$$
\begin{aligned}
\int_{U_{10}} \int_{0}^{2} \gamma(q, t)^{p} \frac{\mathrm{d} t}{t} \mathrm{~d} \mathcal{H}^{n}(q) \leq & \sum_{i \in I_{12}} \int_{R_{i} \cap U_{10}} \int_{0}^{\frac{\text { diam } R_{i}}{2}} \gamma(q, t)^{p} \frac{\mathrm{d} t}{t} \mathrm{~d} \mathcal{H}^{n}(q) \\
& +\sum_{i \in I_{12}} \int_{R_{i} \cap U_{10}} \int_{\frac{\operatorname{diam} R_{i}}{2}}^{2} \gamma(q, t)^{p} \frac{\mathrm{d} t}{t} \mathrm{~d} \mathcal{H}^{n}(q) \\
& +\int_{\pi(\mathcal{Z}) \cap U_{10}} \int_{0}^{2} \gamma(q, t)^{p} \frac{\mathrm{d} t}{t} \mathrm{~d} \mathcal{H}^{n}(q) .
\end{aligned}
$$

We continue the proof of Theorem 7.17 with several lemmas. At first, we prove

Lemma 7.18. There exists some constant $C=C\left(N, n, p, C_{0}\right)$ so that

$$
\sum_{i \in I_{12}} \int_{R_{i} \cap U_{10}} \int_{0}^{\frac{\mathrm{diam} R_{i}}{2}} \gamma(q, t)^{p} \frac{\mathrm{d} t}{t} \mathrm{~d} \mathcal{H}^{n}(q) \leq C \varepsilon^{p} .
$$

Proof. Let $i \in I_{12}, q \in R_{i}, 0<t<\frac{\operatorname{diam} R_{i}}{2}$ and $u \in B(q, t) \cap P_{0} \subset 2 R_{i}$. The function $A$ is in $C^{\infty}\left(2 R_{i}, P_{0}^{\perp}\right)$ (see definition of $A$ on page 78 ). This implies with Lemma A.15 (Taylor's

\footnotetext{
${ }^{2} \tilde{C}$ is given in Lemma $7.20, \hat{C}$ is given in Lemma $7.24 \mathrm{~V}$

${ }^{3} C^{\prime}, C_{1}$ are given in Lemma 7.23
} 
theorem)

$$
\begin{aligned}
A(u)=A(q)+\sum_{l=1}^{n} \partial_{l} A(q) & {\left[\phi^{-1}(u-q)\right]_{l} } \\
& +2 \int_{0}^{1}(1-r)\left\{\sum_{|\kappa|=2} \frac{1}{\kappa !} \partial^{\kappa} A(q+r(u-q))\left(\phi^{-1}(u-q)\right)^{\kappa}\right\} \mathrm{d} r .
\end{aligned}
$$

Let $q_{0} \in B(q, t) \cap P_{0} \subset 2 R_{i}$. With Lemma 6.29, there exists some constant $C=C\left(N, n, C_{0}\right)$ so that, for all $|\kappa|=2$, we have

$$
\left|\partial^{\kappa} A\left(q_{0}\right)\right| \leq \frac{C \varepsilon}{\operatorname{diam} R_{i}} .
$$

With

$$
\left|\left(\phi^{-1}(u-q)\right)^{\kappa}\right|=\prod_{i=1}^{n}\left|\phi^{-1}(u-q)_{i}\right|^{\kappa_{i}} \leq \prod_{i=1}^{n} \underbrace{\left|\phi^{-1}(u-q)\right|^{\kappa_{i}}}_{=|u-q|^{\kappa_{i}} \leq t^{\kappa_{i}}} \leq t^{|\kappa|}=t^{2},
$$

we have

$$
\begin{aligned}
& \gamma(q, t) \\
& =\inf _{a \in \mathcal{A}} \frac{1}{t^{n}} \int_{B(q, t) \cap P_{0}} \frac{d(A(u), a(u))}{t} \mathrm{~d} \mathcal{H}^{n}(u) \\
& \leq \inf _{a \in \mathcal{A}} \frac{1}{t^{n+1}} \mathcal{H}^{n}\left(B(q, t) \cap P_{0}\right) \sup _{u \in B(q, t) \cap P_{0}} d(A(u), a(u)) \\
& =\inf _{a \in \mathcal{A}} \frac{1}{t^{n+1}} \omega_{n} t^{n} \sup _{u \in B(q, t) \cap P_{0}} d(A(u), a(u)) \\
& \stackrel{(7.10)}{\leq} \frac{\omega_{n}}{t} \inf _{a \in \mathcal{A}} \sup _{u \in B(q, t) \cap P_{0}}(\underbrace{\mid A(q)+\sum_{l=1}^{n} \partial_{l} A(q)\left[\phi^{-1}(u-q)\right]_{l}}_{\in \mathcal{A}\left(P_{0}, P_{0}^{\perp}\right)}-a(u) \mid \\
& \left.+\left|2 \int_{0}^{1}(1-r)\left\{\sum_{|\kappa|=2} \frac{1}{\kappa !} \partial^{\kappa} A(q+r(u-q))\left(\phi^{-1}(u-q)\right)^{\kappa}\right\} \mathrm{d} r\right|\right) \\
& =2 \frac{\omega_{n}}{t} \sup _{u \in B(q, t) \cap P_{0}}\left|\int_{0}^{1}(1-r)\left\{\sum_{|\kappa|=2} \frac{1}{\kappa !} \partial^{\kappa} A(q+r(u-q))\left(\phi^{-1}(u-q)\right)^{\kappa}\right\} \mathrm{d} r\right| \\
& \leq \frac{\omega_{n}}{t} \sup _{q_{0} \in B(q, t) \cap P_{0}} \sum_{|\kappa|=2}\left|\partial^{\kappa} A\left(q_{0}\right)\right| \sup _{u \in B(q, t) \cap P_{0}}\left|\left(\phi^{-1}(u-q)\right)^{\kappa}\right| \\
& \stackrel{(7.11)}{\leq} \frac{\omega_{n}}{t} \frac{C \varepsilon}{\operatorname{diam} R_{i}} t^{2}\left(n+\frac{n(n-1)}{2}\right) \\
& =t \frac{C \varepsilon}{\operatorname{diam} R_{i}} \omega_{n} \frac{n(n+1)}{2} \text {. }
\end{aligned}
$$


Hence, with Lemma 6.14, we conclude

$$
\begin{aligned}
& \sum_{i \in I_{12}} \int_{R_{i} \cap U_{10}} \int_{0}^{\frac{\operatorname{diam} R_{i}}{2}} \gamma(q, t)^{p} \frac{\mathrm{d} t}{t} \mathrm{~d} \mathcal{H}^{n}(q) \\
\leq & C \varepsilon^{p} \sum_{i \in I_{12}} \frac{1}{\left(\operatorname{diam} R_{i}\right)^{p}} \int_{R_{i} \cap U_{10}} 1 \mathrm{~d} \mathcal{H}^{n}(q) \int_{0}^{\frac{\operatorname{diam} R_{i}}{2}} t^{p} \frac{\mathrm{d} t}{t} \\
\leq & C \varepsilon^{p} \sum_{i \in I_{12}} \frac{1}{\left(\operatorname{diam} R_{i}\right)^{p}} \mathcal{H}^{n}\left(R_{i}\right) \frac{1}{p}\left(\frac{\operatorname{diam} R_{i}}{2}\right)^{p} \\
\leq & C \varepsilon^{p} \mathcal{H}^{n}\left(U_{12}\right)=C\left(N, n, p, C_{0}\right) \varepsilon^{p} .
\end{aligned}
$$

Now we consider

$$
\sum_{i \in I_{12}} \int_{R_{i} \cap U_{10}} \int_{\frac{\text { diam } R_{i}}{2}}^{2} \gamma(q, t)^{p} \frac{\mathrm{d} t}{t} \mathrm{~d} \mathcal{H}^{n}(q)
$$

and

$$
\int_{\pi(\mathcal{Z}) \cap U_{10}} \int_{0}^{2} \gamma(q, t)^{p} \frac{\mathrm{d} t}{t} \mathrm{~d} \mathcal{H}^{n}(q)
$$

If $q_{1} \in R_{i}$, we get with Lemma 6.14 that $\frac{D\left(q_{1}\right)}{100} \leq \frac{\operatorname{diam} R_{i}}{2}$ and, if $q_{2} \in \pi(\mathcal{Z})$, we obtain with Lemma $6.11 D\left(q_{2}\right)=0$. Hence we conclude using Lemma 6.14

$$
\begin{aligned}
& \sum_{i \in I_{12}} \int_{R_{i} \cap U_{10}} \int_{\frac{\text { diam } R_{i}}{2}}^{2} \gamma(q, t)^{p} \frac{\mathrm{d} t}{t} \mathrm{~d} \mathcal{H}^{n}(q)+\int_{\pi(\mathcal{Z}) \cap U_{10}} \int_{0}^{2} \gamma(q, t)^{p} \frac{\mathrm{d} t}{t} \mathrm{~d} \mathcal{H}^{n}(q) \\
\leq & \sum_{i \in I_{12}} \int_{R_{i} \cap U_{10}} \int_{\frac{D(q)}{100}}^{2} \gamma(q, t)^{p} \frac{\mathrm{d} t}{t} \mathrm{~d} \mathcal{H}^{n}(q)+\int_{\pi(\mathcal{Z}) \cap U_{10}} \int_{\frac{D(q)}{100}}^{2} \gamma(q, t)^{p} \frac{\mathrm{d} t}{t} \mathrm{~d} \mathcal{H}^{n}(q) \\
\leq & \int_{U_{10} \backslash \pi(\mathcal{Z})} \int_{\frac{D(q)}{100}}^{2} \gamma(q, t)^{p} \frac{\mathrm{d} t}{t} \mathrm{~d} \mathcal{H}^{n}(q)+\int_{\pi(\mathcal{Z}) \cap U_{10}} \int_{\frac{D(q)}{100}}^{2} \gamma(q, t)^{p} \frac{\mathrm{d} t}{t} \mathrm{~d} \mathcal{H}^{n}(q) \\
= & \int_{U_{10}} \int_{\frac{D(q)}{100}}^{2} \gamma(q, t)^{p} \frac{\mathrm{d} t}{t} \mathrm{~d} \mathcal{H}^{n}(q) .
\end{aligned}
$$

In the following, we prove some estimate for $\gamma(q, t)$ where $q \in U_{10}$ and $\frac{D(q)}{100}<t<2$. To get this estimate, we need the next lemma.

Lemma 7.19. For all $q \in U_{10}$ and for all $t$ with $\frac{D(q)}{100}<t<2$, there exists some $\tilde{X}=\tilde{X}(q) \in F$ and some $T=T(t)>0$ with

$$
(\tilde{X}, T) \in S, \quad d(\pi(\tilde{X}), q) \leq T \quad \text { and } \quad 20 t \leq T \leq 200 t .
$$

Proof. We have $D(q)=\inf _{(X, s) \in S}(d(\pi(X), q)+s)$, and hence there exists some $(\tilde{X}, \tilde{s}) \in S$ with $d(\pi(\tilde{X}), q)+\tilde{s} \leq D(q)+100 t \leq 200 t$. We set $T:=\min \{40,200 t\}$ which fulfils $20 t \leq T \leq 200 t$ as $t<2$. Using Lemma 6.3 (iv), (v) and $200 t \geq \tilde{s}$, we obtain $(\tilde{X}, T) \in S$.

With $d(\pi(\tilde{X}), q) \leq d(\pi(\tilde{X}), 0)+d(0, q) \leq 5+10$ we get $d(\pi(\tilde{X}), q) \leq T$. 
Now let $q, t, \tilde{X}$ and $T$ as in Lemma 7.19. Furthermore, let $X \in B(\tilde{X}, 200 t) \cap F$. We choose some $n$-dimensional plane named $P^{(q, t, X)}$ with

$$
\beta_{1 ; k}^{P^{(q, t, X)}}(X, t) \leq 2 \beta_{1 ; k}(X, t)
$$

and define

$$
\mathcal{I}(q, t):=\left\{i \in I_{12} \mid R_{i} \cap B(q, t) \neq \emptyset\right\} .
$$

With Lemma 6.14, we have $\left(B(q, t) \cap P_{0}\right) \subset U_{12} \subset \pi(\mathcal{Z}) \cup \bigcup_{i \in I_{12}} R_{i}$ which implies

$$
\left(B(q, t) \cap P_{0}\right) \subset\left((B(q, t) \cap \pi(\mathcal{Z})) \cup \bigcup_{i \in \mathcal{I}(q, t)} B(q, t) \cap R_{i}\right),
$$

and, together with Lemma 7.2, we get

$$
\begin{aligned}
\gamma(q, t) \leq & 3 \frac{1}{t^{n}} \int_{B(q, t) \cap P_{0}} \frac{d\left(u+A(u), P^{(q, t, X)}\right)}{t} \mathrm{~d} \mathcal{H}^{n}(u) \\
\leq & \underbrace{3 \frac{1}{t^{n}} \int_{B(q, t) \cap \pi(\mathcal{Z})} \frac{d\left(u+A(u), P^{(q, t, X)}\right)}{t} \mathrm{~d} \mathcal{H}^{n}(u)}_{=: K_{0}} \\
& +3 \sum_{i \in \mathcal{I}(q, t)} \underbrace{\frac{1}{t^{n}} \int_{B(q, t) \cap R_{i}} \frac{d\left(u+A(u), P^{(q, t, X)}\right)}{t}}_{=: K_{i}} \mathrm{~d} \mathcal{H}^{n}(u) \\
= & K_{0}+3 \sum_{i \in \mathcal{I}(q, t)} K_{i} .
\end{aligned}
$$

At first, we consider $K_{0}$.

Lemma 7.20. There exists some constant $\tilde{C}>1$ so that

$$
\int_{B(q, t) \cap \pi(\mathcal{Z})} d\left(u+A(u), P^{(q, t, X)}\right) \mathrm{d} \mathcal{H}^{n}(u) \leq \int_{B(X, \tilde{C} t) \cap \mathcal{Z}} d\left(x, P^{(q, t, X)}\right) \mathrm{d} \mathcal{H}^{n}(x) .
$$

Proof. Let $g: \pi(\mathcal{Z}) \rightarrow \mathcal{Z}, u \mapsto u+A(u)$. This function is bijective, continuous ( $A$ is $2 \alpha$ Lipschitz on $\pi(Z))$ and $g^{-1}=\left.\pi\right|_{\mathcal{Z}}$ is Lipschitz continuous with Lipschitz constant 1 . With $f(x)=d\left(x, P^{(q, t, X)}\right)$ and $s=n$, we apply [Sch12, Lem. A.1] and get

$$
\begin{aligned}
\int_{B(q, t) \cap \pi(\mathcal{Z})} d\left(u+A(u), P^{(q, t, X)}\right) \mathrm{d} \mathcal{H}^{n}(u) & =\int_{B(q, t) \cap \pi(\mathcal{Z})} f(g(u)) \mathrm{d} \mathcal{H}^{n}(u) \\
& \leq\left(\operatorname{Lip}_{g^{-1}}\right)^{n} \int_{g(B(q, t) \cap \pi(\mathcal{Z}))} f(x) \mathrm{d} \mathcal{H}^{n}(x) \\
& =\int_{g(B(q, t) \cap \pi(\mathcal{Z}))} d\left(x, P^{(q, t, X)}\right) \mathrm{d} \mathcal{H}^{n}(x) .
\end{aligned}
$$

Now it remains to show that there exists some constant $C$ so that

$$
g(B(q, t) \cap \pi(\mathcal{Z})) \subset B(X, C t) \cap \mathcal{Z} .
$$


Let $x \in g(B(q, t) \cap \pi(\mathcal{Z}))$. This implies $x \in \mathcal{Z}$ and so, using Lemma 6.11, we get $d(x)=0$. With (7.13), we conclude

$$
d(\tilde{X})=\inf _{(Y, s) \in S}(d(Y, \tilde{X})+s) \leq d(\tilde{X}, \tilde{X})+T \leq 200 t,
$$

and we obtain with (7.13)

$$
d(\pi(x), \pi(\tilde{X})) \leq d(\pi(x), q)+d(q, \pi(\tilde{X})) \leq t+200 t=201 t .
$$

So, with Lemma 6.12 , we have

$$
d(x, \tilde{X}) \leq 6(d(x)+d(\tilde{X}))+2 d(\pi(x), \pi(\tilde{X})) \leq 1602 t .
$$

We deduce with $\tilde{C}=1802$

$$
d(x, X) \leq d(x, \tilde{X})+d(\tilde{X}, X) \leq 1602 t+200 t=\tilde{C} t
$$

and so $g(B(q, t) \cap \pi(\mathcal{Z})) \subset B(X, \tilde{C} t) \cap \mathcal{Z}$.

Lemma 7.21. There exists some constant $C=C\left(N, n, C_{0}\right)>1$ so that

$$
\int_{B(X, \tilde{C} t) \cap \mathcal{Z}} d\left(x, P^{(q, t, X)}\right) \mathrm{d} \mathcal{H}^{n}(x) \leq C \int_{B(X,(\tilde{C}+1) t)} d\left(x, P^{(q, t, X)}\right) \mathrm{d} \mu(x) .
$$

Proof. At first, we prove for an arbitrary ball $B$ with centre in $\mathcal{Z}$

$$
\mathcal{H}^{n}(\mathcal{Z} \cap B) \leq C\left(N, n, C_{0}\right) \mu(B) .
$$

With [EG92, Dfn. 2.1], we get $\mathcal{H}^{n}(\mathcal{Z} \cap B)=\lim _{\tau \rightarrow 0} \mathcal{H}_{\tau}^{n}(\mathcal{Z} \cap B)$. Let $0<\tau_{0}<\min \left\{\frac{\operatorname{diam} B}{2}, 50\right\}$. We define

$$
\mathcal{F}:=\left\{B(x, s) \mid x \in \mathcal{Z} \cap B, s \leq \tau_{0}\right\} .
$$

With Besicovitch's covering theorem A.12, there exist $N_{0}=N_{0}(N)$ countable families $\mathcal{F}_{j} \subset \mathcal{F}$, $j=1, \ldots, N_{0}$, of disjoint balls with

$$
\mathcal{Z} \cap B \subset \bigcup_{j=1}^{N_{0}} \bigcup_{\tilde{B} \in \mathcal{F}_{j}} \tilde{B} .
$$

For every ball $\tilde{B}=B(x, s) \in \mathcal{F}_{j}$, we have $x \in \mathcal{Z}$ and hence, using the definition of $\mathcal{Z}$ (see page 66), we deduce $h(x)=0$. With $h(x)=0<s<50$ and Lemma 6.3 (iv), we get $(x, s) \in S \subset S_{\text {total }}$ and so

$$
\left(\frac{\operatorname{diam} \tilde{B}}{2}\right)^{n} \leq 2 \frac{\mu(\tilde{B})}{\delta} .
$$

The centre of $B$ is also in $\mathcal{Z}$ and hence, analogously, we conclude

$$
\left(\frac{\operatorname{diam} B}{2}\right)^{n} \leq 2 \frac{\mu(B)}{\delta}
$$


With (B) from page 64, we get

$$
\mu(2 B) \stackrel{(\mathrm{B})}{\leq} C_{0}(\operatorname{diam} 2 B)^{n}=4^{n} C_{0}\left(\frac{\operatorname{diam} B}{2}\right)^{n} \leq 4^{n} C_{0} \frac{2}{\delta} \mu(B) .
$$

Since $x \in B$ and $s \leq \tau_{0}<\frac{\operatorname{diam} B}{2}$, we obtain

$$
\tilde{B}=B(x, s) \subset 2 B .
$$

Now, by definition of $\mathcal{H}_{\tau_{0}}^{n}$ [EG92, Dfn. 2.1] and because $\delta=\delta(N, n)$ (see (6.2) on page 65), we deduce

$$
\begin{aligned}
\mathcal{H}_{\tau_{0}}^{n}(\mathcal{Z} \cap B) & \leq \sum_{j=1}^{N_{0}} \sum_{\tilde{B} \in \mathcal{F}_{j}} \omega_{n}\left(\frac{\operatorname{diam} \tilde{B}}{2}\right)^{n} \\
& \leq 2 \sum_{j=1}^{N_{0}} \sum_{\tilde{B} \in \mathcal{F}_{j}} \omega_{n} \frac{\mu(\tilde{B})}{\delta} \\
& \leq 2 \frac{\omega_{n}}{\delta} \sum_{j=1}^{N_{0}} \mu(2 B) \\
& \stackrel{(7.17)}{\leq} C\left(N, n, C_{0}\right) \mu(B) .
\end{aligned}
$$

So, with $\tau_{0} \rightarrow 0$, the inequality (7.16) is proven.

Let $\tilde{C}$ be the constant from Lemma 7.20. For an arbitrary $0<\sigma \leq t$, we define

$$
\mathcal{G}_{\sigma}:=\{B(x, s) \mid x \in \mathcal{Z} \cap B(X, \tilde{C} t), s \leq \sigma\} .
$$

With Besicovitch's covering theorem A.12, there exist $N_{0}$ families $\mathcal{G}_{\sigma, j} \subset \mathcal{G}_{\sigma}$ of disjoint balls, where $j=1, . ., N_{0}$, with

$$
\mathcal{Z} \cap B(X, \tilde{C} t) \subset \bigcup_{j=1}^{N_{0}} \bigcup_{B \in \mathcal{G}_{\sigma, j}}(\mathcal{Z} \cap B)
$$

and where $N_{0}$ depends only on $N$. We denote by $p_{B}$ the centre of the ball $B$ and conclude

$$
\begin{aligned}
& \int_{\mathcal{Z} \cap B(X, \tilde{C} t)} d\left(x, P^{(q, t, X)}\right) \mathrm{d} \mathcal{H}^{n}(x) \\
\leq & \sum_{j=1}^{N_{0}} \sum_{B \in \mathcal{G}_{\sigma, j}} \int_{\mathcal{Z} \cap B} d\left(x, p_{B}\right)+d\left(p_{B}, P^{(q, t, X)}\right) \mathrm{d} \mathcal{H}^{n}(x) \\
\leq & \sum_{j=1}^{N_{0}} \sum_{B \in \mathcal{G}_{\sigma, j}} \int_{\mathcal{Z} \cap B} \sigma+d\left(p_{B}, P^{(q, t, X)}\right) \mathrm{d} \mathcal{H}^{n}(x) \\
= & \sum_{j=1}^{N_{0}} \sum_{B \in \mathcal{G}_{\sigma, j}}\left(\sigma+d\left(p_{B}, P^{(q, t, X)}\right)\right) \mathcal{H}^{n}(\mathcal{Z} \cap B)
\end{aligned}
$$




$$
\begin{aligned}
& \stackrel{\text { (7.16) }}{\leq} \sum_{j=1}^{N_{0}} \sum_{B \in \mathcal{G}_{\sigma, j}}\left(\sigma+d\left(p_{B}, P^{(q, t, X)}\right)\right) C(N, n) \mu(B) \\
& =C\left(N, n, C_{0}\right) \sum_{j=1}^{N_{0}} \sum_{B \in \mathcal{G}_{\sigma, j}} \int_{B}\left(\sigma+d\left(p_{B}, P^{(q, t, X)}\right)\right) \mathrm{d} \mu(x) \\
& \leq C\left(N, n, C_{0}\right) \sum_{j=1}^{N_{0}} \sum_{B \in \mathcal{G}_{\sigma, j}} \int_{B}\left(\sigma+d\left(p_{B}, x\right)+d\left(x, P^{(q, t, X)}\right)\right) \mathrm{d} \mu(x) \\
& \leq C\left(N, n, C_{0}\right) \sum_{j=1}^{N_{0}} \sum_{B \in \mathcal{G}_{\sigma, j}} \int_{B}\left(2 \sigma+d\left(x, P^{(q, t, X)}\right)\right) \mathrm{d} \mu(x) \\
& \leq C\left(N, n, C_{0}\right)\left(\mu(B(X,(\tilde{C}+1) t)) 2 \sigma+\int_{B(X,(\tilde{C}+1) t)} d\left(x, P^{(q, t, X)}\right) \mathrm{d} \mu(x)\right) .
\end{aligned}
$$

With $\sigma \rightarrow 0$, the assertion holds.

With Lemma 7.20 and Lemma 7.21, we get for $K_{0}$ using that $k \geq \tilde{k} \geq \tilde{C}+1$, where $\tilde{k}$ is defined on page 103

$$
\begin{aligned}
K_{0} & =3 \frac{1}{t^{n}} \int_{B(q, t) \cap \pi(\mathcal{Z})} \frac{d\left(u+A(u), P^{(q, t, X)}\right)}{t} \mathrm{~d} \mathcal{H}^{n}(u) \\
& \leq \frac{3}{t^{n+1}} \int_{B(X, \tilde{C} t) \cap \mathcal{Z}} d\left(x, P^{(q, t, X)}\right) \mathrm{d} \mathcal{H}^{n}(x) \\
& \leq C\left(N, n, C_{0}\right) \frac{1}{t^{n+1}} \int_{B(X,(\tilde{C}+1) t)} d\left(x, P^{(q, t, X)}\right) \mathrm{d} \mu(x) \\
& \leq C\left(N, n, C_{0}\right) \beta_{1 ; k}^{P^{(q, t, X)}}(X, t) \\
& (7.14) \\
& \leq C\left(N, n, C_{0}\right) \beta_{1 ; k}(X, t) .
\end{aligned}
$$

To estimate $K_{i}$, we need the following lemma.

Lemma 7.22. There exists some constant $C_{4}=C_{4}\left(N, n, C_{0}\right)>1$ so that, for all $i \in I_{12}$ and $u \in R_{i}$, we have

$$
d\left(\pi_{P_{i}}(u+A(u)), B_{i}\right) \leq C_{4} \operatorname{diam} R_{i} .
$$

We recall that $P_{i}$ is the $n$-dimensional plane, which is, in the sense of Lemma 6.3 (vi), associated to the ball $B\left(X_{i}, t_{i}\right)=B_{i}$ given by Lemma 6.15 (cf. Definition 6.18).

Proof. For every $i \in I_{12} \subset I$, we have with Lemma 6.15 that $B_{i}=B\left(X_{i}, t_{i}\right)$ and $\left(X_{i}, t_{i}\right) \in$ $S \subset S_{\text {total }}$. Hence we can use Lemma 5.10 (ii) $\left(\sigma=2 \varepsilon, x=X_{i}, t=t_{i}, \lambda=\frac{\delta}{2}, P=P_{i}\right)$ to get some $y \in 2 B_{i} \cap P_{i}$, where $P_{i}=P_{\left(X_{i}, t_{i}\right)}$. We obtain with Corollary $2.24\left(P_{1}=P_{j}, P_{2}=P_{0}\right)$, $\alpha \leq \tilde{\alpha}<\frac{1}{2}$ ( $\tilde{\alpha}$ is defined on page 103) and Lemma 6.15

$$
d\left(u+A_{i}(u), y\right) \leq \frac{1}{1-\alpha} d(u, \pi(y))<2[d\left(u, \pi\left(X_{i}\right)\right)+\underbrace{d\left(\pi\left(X_{i}\right), \pi(y)\right)}_{\leq d\left(X_{i}, y\right) \leq \operatorname{diam} B_{i} \leq 200 \operatorname{diam} R_{i}}] \leq C \operatorname{diam} R_{i} .
$$




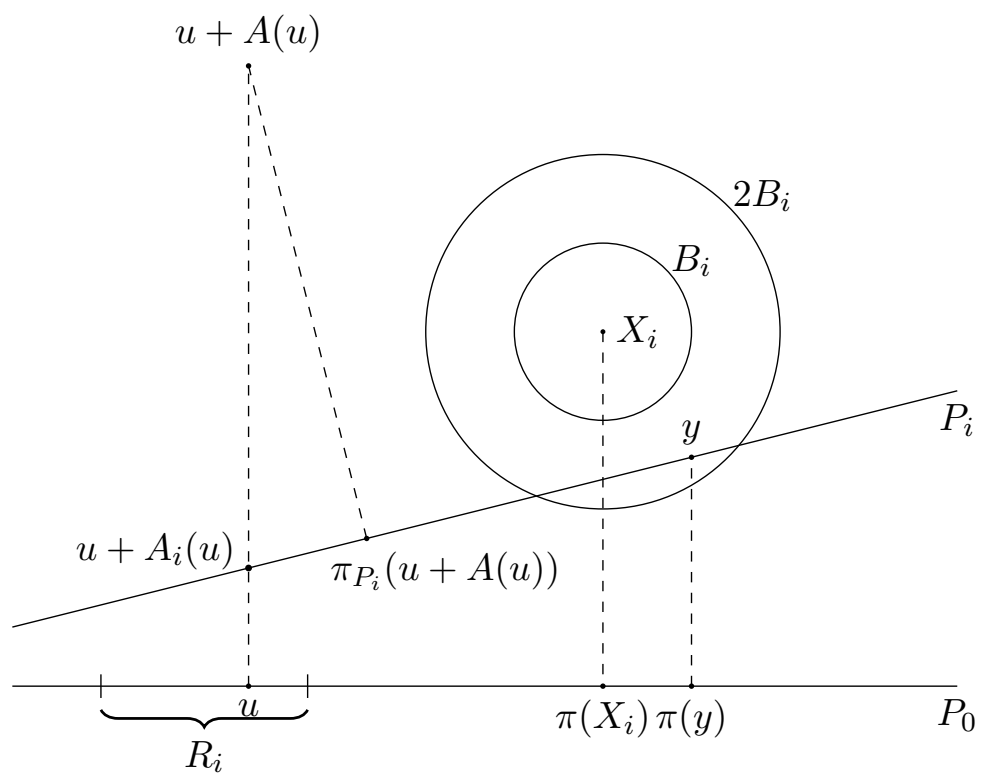

Figure 7.1: $d\left(\pi_{P_{i}}(u+A(u)), B_{i}\right) \leq C \operatorname{diam} R_{i}$

Moreover, with Lemma 6.22 (iv) and $\varepsilon \leq \tilde{\varepsilon} \leq 1$ ( $\tilde{\varepsilon}$ is defined on page 103), we get

$$
d(\pi_{P_{i}}(u+A(u)), \underbrace{u+A_{i}(u)}_{=\pi_{P_{i}}\left(u+A_{i}(u)\right)}) \leq d\left(u+A(u), u+A_{i}(u)\right)=d\left(A(u), A_{i}(u)\right) \leq C \operatorname{diam} R_{i}
$$

for some $C=C\left(N, n, C_{0}\right)$. Using these estimates, we conclude

$$
\begin{aligned}
d\left(\pi_{P_{i}}(u+A(u)), B_{i}\right) & \leq d\left(\pi_{P_{i}}(u+A(u)), u+A_{i}(u)\right)+d\left(u+A_{i}(u), y\right)+d\left(y, B_{i}\right) \\
& \leq C \operatorname{diam} R_{i} .
\end{aligned}
$$

Now, with Lemma 7.22 and $K_{i}$ from (7.15), we obtain for $i \in \mathcal{I}(q, t) \subset I_{12}$

$$
\begin{aligned}
K_{i}= & \frac{1}{t^{n}} \int_{B(q, t) \cap R_{i}} \frac{d\left(u+A(u), P^{(q, t, X)}\right)}{t} \mathrm{~d} \mathcal{H}^{n}(u) \\
\leq & \frac{1}{t^{n}} \int_{B(q, t) \cap R_{i}} \frac{d\left(u+A(u), P_{i}\right)+d\left(\pi_{P_{i}}(u+A(u)), P^{(q, t, X)}\right)}{t} \mathrm{~d} \mathcal{H}^{n}(u) \\
\leq & \frac{1}{t^{n}} \int_{B(q, t) \cap R_{i}} \frac{d\left(u+A(u), P_{i}\right)}{t} \mathrm{~d} \mathcal{H}^{n}(u) \\
& +\frac{1}{t^{n}} \sup \left\{\frac{d\left(\pi_{P_{i}}(v+A(v)), P^{(q, t, X)}\right)}{t} \mid v \in B(q, t) \cap R_{i}\right\} \mathcal{H}^{n}\left(B(q, t) \cap R_{i}\right)
\end{aligned}
$$




$$
\begin{array}{ll}
\stackrel{\text { L. } .22}{\leq} & \frac{1}{t^{n}} \int_{B(q, t) \cap R_{i}} \frac{d\left(u+A(u), P_{i}\right)}{t} \mathrm{~d} \mathcal{H}^{n}(u) \\
& +\omega_{n}\left(\frac{\operatorname{diam} R_{i}}{t}\right)^{n} \sup \left\{\frac{d\left(w, P^{(q, t, X)}\right)}{t} \mid w \in P_{i}, d\left(w, B_{i}\right) \leq C_{4} \operatorname{diam} R_{i}\right\} .
\end{array}
$$

Since $P_{i}$ is the graph of $A_{i}$, we get for any $u \in B(q, t) \cap R_{i}$ with Lemma 6.22 (iv) that there exists some $\bar{C}=\bar{C}\left(N, n, C_{0}\right)$ with

$$
d\left(u+A(u), P_{i}\right) \leq d\left(u+A(u), u+A_{i}(u)\right)=d\left(A(u), A_{i}(u)\right) \leq \bar{C} \varepsilon \operatorname{diam} R_{i},
$$

and so, using Lemma A.7,

$$
\begin{aligned}
\frac{1}{t^{n}} \int_{B(q, t) \cap R_{i}} \frac{d\left(u+A(u), P_{i}\right)}{t} \mathrm{~d} \mathcal{H}^{n}(u) & \leq \bar{C} \varepsilon \frac{\operatorname{diam} R_{i}}{t^{n+1}} \int_{R_{i}} 1 \mathrm{~d} \mathcal{H}^{n}(u) \\
& =C\left(N, n, C_{0}\right) \varepsilon(\sqrt{n})^{-n}\left(\frac{\operatorname{diam} R_{i}}{t}\right)^{n+1} .
\end{aligned}
$$

Lemma 7.23. There exists some constant $C=C\left(N, n, C_{0}\right)$ so that for all $i \in \mathcal{I}(q, t)$

$$
\begin{aligned}
& \sup \left\{\frac{d\left(w, P^{(q, t, X)}\right)}{t} \mid w \in P_{i}, d\left(w, B_{i}\right) \leq C_{4} \operatorname{diam} R_{i}\right\} \\
\leq & C \varepsilon \frac{\operatorname{diam} R_{i}}{t}+C \frac{1}{t}\left(\frac{1}{\left(\operatorname{diam} R_{i}\right)^{n}} \int_{2 B_{i}} d\left(z, P^{(q, t, X)}\right)^{\frac{1}{3}} \mathrm{~d} \mu(z)\right)^{3} .
\end{aligned}
$$

Proof. Let $i \in \mathcal{I}(q, t)$. Due to the construction of $B_{i}=B\left(X_{i}, t_{i}\right)$ (see Lemma 6.15), we have $\left(X_{i}, t_{i}\right) \in S \subset S_{\text {total }}$ and so $\delta\left(X_{i}, t_{i}\right) \geq \frac{\delta}{2}$. With Corollary $5.3\left(\lambda=\frac{\delta}{2}, B(x, t)=B\left(X_{i}, t_{i}\right)\right.$, $\left.\Upsilon=\mathbb{R}^{N}\right)$, there exist constants $C_{1}=C_{1}\left(N, n, C_{0}\right)>3, C_{2}=C_{2}\left(N, n, C_{0}\right)>1$ and some $\left(n, 10 n \frac{t_{i}}{C_{1}}\right)$-simplex $T=\Delta\left(x_{0}, \ldots, x_{n}\right) \in F \cap B_{i}$ with

$$
\mu\left(B\left(x_{\kappa}, \frac{t_{i}}{C_{1}}\right) \cap B_{i}\right) \geq \frac{t_{i}^{n}}{C_{2}}
$$

for all $\kappa=0, \ldots, n$ and, because $C_{1}>3$ and $k \geq \tilde{k} \geq 2(\tilde{k}$ is defined on page 103), we have

$$
B\left(x_{\kappa}, \frac{t_{i}}{C_{1}}\right) \subset 2 B_{i} \subset k B_{i}=B\left(X_{i}, k t_{i}\right)
$$

We set $C^{\prime}:=400 C_{2}, \tilde{B}_{\kappa}:=B\left(x_{\kappa}, \frac{t_{i}}{C_{1}}\right)$ and define for all $\kappa=0, \ldots, n$

$$
Z_{\kappa}:=\left\{z \in \tilde{B}_{\kappa} \cap F \mid d\left(z, P_{i}\right) \leq C^{\prime} \varepsilon \operatorname{diam} R_{i}\right\}
$$

We have $\left(X_{i}, t_{i}\right) \in S_{\text {total }}$ and hence $\beta_{1 ; k}^{P_{i}}\left(X_{i}, t_{i}\right) \leq 2 \varepsilon$. Using this and Lemma 6.15 (i), we 
obtain

$$
\begin{aligned}
\mu\left(\tilde{B}_{\kappa} \backslash Z_{\kappa}\right) & =\frac{1}{C^{\prime} \varepsilon \operatorname{diam} R_{i}} \int_{\tilde{B}_{\kappa} \backslash Z_{\kappa}} C^{\prime} \varepsilon \operatorname{diam} R_{i} \mathrm{~d} \mu(z) \\
& <\frac{1}{C^{\prime} \varepsilon \operatorname{diam} R_{i}} \int_{\tilde{B}_{\kappa} \backslash Z_{\kappa}} d\left(z, P_{i}\right) \mathrm{d} \mu(z) \\
& \stackrel{(7.22)}{\leq} \frac{1}{C^{\prime} \varepsilon \operatorname{diam} R_{i}} \int_{B\left(X_{i}, k t_{i}\right)} d\left(z, P_{i}\right) \mathrm{d} \mu(z) \\
& =\frac{t_{i}^{n+1}}{C^{\prime} \varepsilon \operatorname{diam} R_{i}} \frac{1}{t_{i}^{n}} \int_{k B_{i}} \frac{d\left(z, P_{i}\right)}{t_{i}} \mathrm{~d} \mu(z) \\
& =\frac{t_{i}^{n+1}}{C^{\prime} \varepsilon \operatorname{diam} R_{i}} \beta_{1 ; k}^{P_{i}}\left(X_{i}, t_{i}\right) \\
& \leq \frac{t_{i}^{n+1} 100}{C^{\prime} \varepsilon t_{i}} 2 \varepsilon=\frac{t_{i}^{n}}{2 C_{2}} .
\end{aligned}
$$

Using Lemma 6.15 (i) again, we get

$$
\mu\left(Z_{\kappa}\right) \geq \mu\left(\tilde{B}_{\kappa}\right)-\mu\left(\tilde{B}_{\kappa} \backslash Z_{\kappa}\right) \stackrel{(7.21)}{\geq} \frac{t_{i}^{n}}{C_{2}}-\frac{t_{i}^{n}}{2 C_{2}}=\frac{t_{i}^{n}}{2 C_{2}} \geq \frac{\operatorname{diam} R_{i}^{n}}{2^{n+1} C_{2}}>0 .
$$

For all $\kappa \in\{0, \ldots, n\}$, let $z_{\kappa} \in Z_{\kappa} \subset \tilde{B}_{\kappa}$ and set $y_{\kappa}:=\pi_{P_{i}}\left(z_{\kappa}\right)$. Since $\varepsilon \leq \tilde{\varepsilon} \leq \frac{1}{2 C^{\prime} C_{1}}(\tilde{\varepsilon}$ was chosen on page 103), we deduce

$$
d\left(y_{\kappa}, x_{\kappa}\right) \leq d\left(y_{\kappa}, z_{\kappa}\right)+d\left(z_{\kappa}, x_{\kappa}\right) \leq d\left(z_{\kappa}, P_{i}\right)+\frac{t_{i}}{C_{1}} \stackrel{(7.23)}{\leq} C^{\prime} \varepsilon \operatorname{diam} R_{i}+\frac{t_{i}}{C_{1}} \leq 2 \frac{t_{i}}{C_{1}} .
$$

Due to Lemma 2.14, the simplex $S=\Delta\left(y_{0}, \ldots, y_{n}\right)$ is an $\left(n, 6 n \frac{t_{i}}{C_{1}}\right)$-simplex and, using the triangle inequality, we obtain $S \subset 2 B_{i}$. Now, with Lemma 2.27, $\left(C=\frac{C_{1}}{6 n}, \hat{C}=2, t=t_{i}\right.$, $\left.m=n, x=X_{i}\right)$ there exists some orthonormal basis $\left(o_{1}, \ldots, o_{n}\right)$ of $\operatorname{span}\left(y_{1}-y_{0}, \ldots, y_{n}-y_{0}\right)=$ $P_{i}-y_{0}$ and there exists $\gamma_{l, r} \in \mathbb{R}$ with

$$
o_{l}=\sum_{r=1}^{l} \gamma_{l, r}\left(y_{r}-y_{0}\right)
$$

and

$$
\left|\gamma_{l, r}\right| \leq\left(2 n \frac{C_{1}}{6 n} 2\right)^{n} \frac{C_{1}}{6 n t_{i}}=\left(\frac{2 C_{1}}{3}\right)^{n} \frac{C_{1}}{6 n t_{i}}
$$

for all $1 \leq l \leq n$ and $1 \leq r \leq l$.

Now let $w \in P_{i}$ with $d\left(w, B_{i}\right) \leq C_{4} \operatorname{diam} R_{i}$. We obtain

$$
w-y_{0}=\sum_{\kappa=1}^{n}\left\langle w-y_{0}, o_{\kappa}\right\rangle o_{\kappa}=\sum_{\kappa=1}^{n}\left\langle w-y_{0}, o_{\kappa}\right\rangle \sum_{r=1}^{\kappa} \gamma_{\kappa, r}\left(y_{r}-y_{0}\right)
$$


and so, with Lemma $2.3\left(b=w, P=P^{(q, t, X)}\right)$ and $\left|w-y_{0}\right|=d\left(w, y_{0}\right) \leq d\left(w, B_{i}\right)+\operatorname{diam} B_{i}+$ $d\left(B_{i}, y_{0}\right) \leq C t_{i}$, we get

$$
\begin{aligned}
& \quad d\left(w, P^{(q, t, X)}\right) \\
& \stackrel{(7.25)}{\leq} d\left(y_{0}, P^{(q, t, X)}\right)+\sum_{\kappa=1}^{n} \underbrace{\mid\left\langle w-y_{0}, o_{\kappa}\right\rangle}_{\leq\left|w-y_{0}\right|} \sum_{r=1}^{\kappa}\left|\gamma_{\kappa, r}\right|\left(d\left(y_{r}, P^{(q, t, X)}\right)+d\left(y_{0}, P^{(q, t, X)}\right)\right) \\
& \leq d\left(y_{0}, P^{(q, t, X)}\right)+\sum_{\kappa=1}^{n} C t_{i} \sum_{r=0}^{n}\left(\frac{2 C_{1}}{3}\right)^{n} \frac{C_{1}}{6 n t_{i}}\left(d\left(y_{r}, P^{(q, t, X)}\right)+d\left(y_{0}, P^{(q, t, X)}\right)\right) \\
& \leq n C C_{1}^{n+1} \sum_{r=0}^{n} d\left(y_{r}, P^{(q, t, X)}\right) \\
& \leq n C C_{1}^{n+1} \sum_{r=1}^{n}\left(d\left(y_{r}, z_{r}\right)+d\left(z_{r}, P^{(q, t, X)}\right)\right) \\
& \leq n C C_{1}^{n+1} \sum_{r=0}^{n} d\left(z_{r}, P_{i}\right)+n C C_{1}^{n+1} \sum_{r=0}^{n} d\left(z_{r}, P^{(q, t, X)}\right) \\
& \stackrel{(7.23)}{\leq} n^{2} C C_{1}^{n+1} C^{\prime} \varepsilon \operatorname{diam} R_{i}+n C C_{1}^{n+1} \sum_{r=0}^{n} d\left(z_{r}, P^{(q, t, X)}\right) .
\end{aligned}
$$

The previous results are valid for arbitrary $z_{\kappa} \in Z_{\kappa}$, hence we get

$$
\begin{aligned}
& d\left(w, P^{(q, t, X)}\right)-n^{2} C C_{1}^{n+1} C^{\prime} \varepsilon \operatorname{diam} R_{i} \\
&=\left(\frac{1}{\prod_{r=0}^{n} \mu\left(Z_{r}\right)} \int_{Z_{0}} \ldots \int_{Z_{n}}\left(d\left(w, P^{(q, t, X)}\right)-n^{2} C C_{1}^{n+1} C^{\prime} \varepsilon \operatorname{diam} R_{i}\right)^{\frac{1}{3}} \mathrm{~d} \mu\left(z_{n}\right) \ldots \mathrm{d} \mu\left(z_{0}\right)\right)^{3} \\
& \stackrel{(7.26)}{\leq}\left(\frac{1}{\prod_{r=0}^{n} \mu\left(Z_{r}\right)} \int_{Z_{0}} \ldots \int_{Z_{n}}\left(n C C_{1}^{n+1} \sum_{r=0}^{n} d\left(z_{r}, P^{(q, t, X)}\right)\right)^{\frac{1}{3}} \mathrm{~d} \mu\left(z_{n}\right) \ldots \mathrm{d} \mu\left(z_{0}\right)\right)^{3} \\
& \leq n C C_{1}^{n+1}\left(\frac{1}{\prod_{r=1}^{n} \mu\left(Z_{r}\right)} \int_{Z_{0}} \ldots \int_{Z_{n}} \sum_{r=0}^{n} d\left(z_{r}, P^{(q, t, X)}\right)^{\frac{1}{3}} \mathrm{~d} \mu\left(z_{n}\right) \ldots \mathrm{d} \mu\left(z_{0}\right)\right)^{3} \\
&= n C C_{1}^{n+1}\left(\sum_{r=0}^{n} \frac{1}{\mu\left(Z_{r}\right)} \int_{Z_{r}} d\left(z_{r}, P^{(q, t, X)}\right)^{\frac{1}{3}} \mathrm{~d} \mu\left(z_{r}\right)\right)^{3} \\
& \stackrel{(7.24)}{\leq} n C C_{1}^{n+1}\left(\sum_{r=0}^{n} \frac{2^{n+1} C_{2}}{\operatorname{diam} R_{i}^{n}} \int_{Z_{r}} d\left(z_{r}, P^{(q, t, X)}\right)^{\frac{1}{3}} \mathrm{~d} \mu\left(z_{r}\right)\right)^{3} \\
&= n C C_{1}^{n+1}\left(\frac{2^{n+1} C_{2}}{\operatorname{diam} R_{i}^{n}} \int_{\bigcup_{r=0}^{n} Z_{r}} d\left(z, P^{(q, t, X)}\right)^{\frac{1}{3}} \mathrm{~d} \mu(z)\right)^{3} \\
& \stackrel{(7.22)}{\leq} n C C_{1}^{n+1}\left(\frac{2^{n+1} C_{2}}{\operatorname{diam} R_{i}^{n}} \int_{2 B_{i}} d\left(z, P^{(q, t, X)}\right)^{\frac{1}{3}} \mathrm{~d} \mu(z)\right)^{3},
\end{aligned}
$$


where we used that the sets $Z_{r}$ are disjoint. This implies

$$
\begin{aligned}
\frac{d\left(w, P^{(q, t, X)}\right)}{t} \leq & n^{2} C C_{1}^{n+1} C^{\prime} \varepsilon \frac{\operatorname{diam} R_{i}}{t} \\
& +n C C_{1}^{n+1} \frac{\left(2^{n+1} C_{2}\right)^{3}}{t}\left(\frac{1}{\left(\operatorname{diam} R_{i}\right)^{n}} \int_{2 B_{i}} d\left(z, P^{(q, t, X)}\right)^{\frac{1}{3}} \mathrm{~d} \mu(z)\right)^{3} .
\end{aligned}
$$

Since $w \in P_{i}$ was arbitrarily chosen with $d\left(w, B_{i}\right) \leq C_{4}$ diam $R_{i}$, we get the statement with $C=C\left(N, n, C_{0}\right):=n C C_{1}^{n+1} \cdot \max \left\{n C^{\prime},\left(2^{n+1} C_{2}\right)^{3}\right\}$.

Lemma 7.24. There exists some constant $C=C\left(n, C_{0}\right)$ so that

$$
\sum_{i \in \mathcal{I}(q, t)}\left(\frac{\operatorname{diam} R_{i}}{t}\right)^{n} \frac{1}{t}\left(\frac{1}{\left(\operatorname{diam} R_{i}\right)^{n}} \int_{2 B_{i}} d\left(z, P^{(q, t, X)}\right)^{\frac{1}{3}} \mathrm{~d} \mu(z)\right)^{3} \leq C \beta_{1 ; k}(X, t) .
$$

Proof. Let $i \in \mathcal{I}(q, t)\left(\mathcal{I}(q, t)\right.$ is defined on page 106) and $x \in 2 B_{i}$. We define

$$
\begin{aligned}
J(i) & :=\left\{j \in \mathcal{I}(q, t) \mid \operatorname{diam} B_{j} \leq \operatorname{diam} B_{i}, 2 B_{i} \cap 2 B_{j} \neq \emptyset\right\}, \\
\Xi_{i}(x) & :=\sum_{j \in J(i)} \chi_{2 B_{j}}(x) .
\end{aligned}
$$

At first, we prove some intermediate results:

I. There exists some constant $C=C\left(n, C_{0}\right)$ so that, for all $i \in \mathcal{I}(q, t)$, we have

$$
\int_{2 B_{i}} \Xi_{i}(x) \mathrm{d} \mu(x) \leq C\left(\operatorname{diam} R_{i}\right)^{n} .
$$

This implies that $\Xi_{i}(x)<\infty$ for $\mu$-almost all $x \in 2 B_{i}$.

Proof. Let $i \in \mathcal{I}(q, t)$. At first, we prove that there exists some constant $C=C(n)>1$ so that

$$
\sum_{j \in J(i)}\left(\operatorname{diam} R_{j}\right)^{n} \leq C\left(\operatorname{diam} R_{i}\right)^{n}
$$

Let $j \in J(i)$. With Lemma 6.15 (i) applied to $j$ and the definition of $J(i)$, we deduce

$$
\operatorname{diam} R_{j} \leq \operatorname{diam} B_{j} \leq \operatorname{diam} B_{i} \leq 200 \operatorname{diam} R_{i} .
$$

Using Lemma 6.15 (i), (ii), we get

$$
\begin{aligned}
d\left(R_{i}, R_{j}\right) & \leq d\left(R_{i}, \pi\left(B_{i}\right)\right)+\operatorname{diam} B_{i}+\underbrace{d\left(\pi\left(B_{i}\right), \pi\left(B_{j}\right)\right)}_{<d\left(2 B_{i}, 2 B_{j}\right)+\operatorname{diam} B_{i}+\operatorname{diam} B_{j}}+\operatorname{diam} B_{j}+d\left(\pi\left(B_{j}\right), R_{j}\right) \\
& \leq C \operatorname{diam} R_{i} .
\end{aligned}
$$


This implies for some large enough constant $C>1$ that $R_{j} \subset C R_{i}$. Since the cubes $\stackrel{\circ}{R}_{j}$ are disjoint (see Lemma 6.14 (ii)), we get with Lemma A.7

$$
\sum_{j \in J(i)}\left(\operatorname{diam} R_{j}\right)^{n}=\sum_{j \in J(i)}(\sqrt{n})^{n} \mathcal{H}^{n}\left(R_{j}\right) \leq(\sqrt{n})^{n} \mathcal{H}^{n}\left(C R_{i}\right)=C(n)\left(\operatorname{diam} R_{i}\right)^{n} .
$$

In the following, we apply Fatou's Lemma [EG92, 1.3, Thm.1] to interchange the integration with the summation. With (B) from page 64 and Lemma 6.15 (i), we obtain

$$
\begin{aligned}
\int_{2 B_{i}} \Xi_{i}(x) \mathrm{d} \mu(x) & =\int_{2 B_{i}} \sum_{j \in J(i)} \chi_{2 B_{j}}(x) \mathrm{d} \mu(x) \\
& \leq \sum_{j \in J(i)} \int_{2 B_{i}} \chi_{2 B_{j}}(x) \mathrm{d} \mu(x) \\
& \leq \sum_{j \in J(i)} \mu\left(2 B_{j}\right) \\
& \text { (B) } 2^{n} C_{0} \sum_{j \in J(i)}\left(\operatorname{diam} B_{j}\right)^{n} \\
& \leq C\left(n, C_{0}\right) \sum_{j \in J(i)}\left(\operatorname{diam} R_{j}\right)^{n} \\
& \leq C\left(n, C_{0}\right)\left(\operatorname{diam} R_{i}\right)^{n} .
\end{aligned}
$$

II. Let $x \in \mathbb{R}^{N}$ and $m \in \mathbb{N}$. There exists some $C=C(n)>1$ with

$$
\sum_{\substack{i \in \mathcal{I}(q, t) \\ \Xi_{i}(x)=m}} \chi_{2 B_{i}}(x) \leq C .
$$

Proof. Let $l, o \in \mathcal{I}(q, t)$ with $x \in 2 B_{l} \cap 2 B_{o}$ and

$$
\Xi_{l}(x)=m=\Xi_{o}(x) .
$$

Without loss of generality, we have $\operatorname{diam} B_{l} \leq \operatorname{diam} B_{o}$.

Assume that diam $B_{l}<\operatorname{diam} B_{o}$. We define

$$
J(l, x):=\left\{\iota \in J(l) \mid x \in 2 B_{\iota}\right\} .
$$

Let $j \in J(l, x)$. By definition of $J(l)$, we get $\operatorname{diam} B_{j} \leq \operatorname{diam} B_{l}<\operatorname{diam} B_{o}$ and $x \in 2 B_{j}$. Since $x \in 2 B_{o}$, it follows $2 B_{o} \cap 2 B_{j} \neq \emptyset$ and, because diam $B_{j}<\operatorname{diam} B_{o}$, we get $j \in J(o, x)$. Furthermore, we have $o \in J(o, x)$, but $o \notin J(l, x)$ because by our assumption we have $\operatorname{diam} B_{l}<\operatorname{diam} B_{o}$. So we get

$$
J(l, x) \subsetneq J(o, x) .
$$


Now we obtain a contradiction

$$
m=\Xi_{l}(x)=\sum_{j \in J(l)} \chi_{2 B_{j}}(x)=\sum_{j \in J(l, x)} \chi_{2 B_{j}}(x)<\sum_{j \in J(o, x)} \chi_{2 B_{j}}(x)=\Xi_{o}(x)=m .
$$

Hence there exists some $\lambda=\lambda(x, m) \in(0, \infty)$ so that, for $l \in \mathcal{I}(q, t)$ with $x \in 2 B_{l}$ and $\Xi_{l}(x)=m$, we have

$$
\operatorname{diam} B_{l}=\lambda,
$$

and, we obtain with Lemma 6.15 (i), (ii)

$$
\lambda \leq 200 \operatorname{diam} R_{l} \leq 200 \lambda \quad \text { and } \quad d\left(R_{l}, \pi\left(B_{l}\right)\right) \leq 100 \lambda .
$$

Using

$$
d\left(R_{l}, \pi(x)\right) \leq d\left(R_{l}, \pi\left(B_{l}\right)\right)+2 \operatorname{diam} B_{l} \leq 102 \lambda,
$$

we get $R_{l} \subset B(\pi(x), 103 \lambda) \cap P_{0}$. With Lemma A.7, we have

$$
\mathcal{H}^{n}\left(R_{l}\right)=(\sqrt{n})^{-n}\left(\operatorname{diam} R_{l}\right)^{n} \geq(\sqrt{n})^{-n}\left(\frac{1}{200} \lambda\right)^{n}
$$

and, according to Lemma 6.14 (ii) the cubes $R_{l}$ have disjoint interior which implies that there are at most

$$
\frac{\mathcal{H}^{n}\left(B(\pi(x), 103 \lambda) \cap P_{0}\right)}{(\sqrt{n})^{-n}\left(\frac{1}{200} \lambda\right)^{n}}=\frac{\omega_{n}(103 \lambda)^{n}}{(\sqrt{n})^{-n}\left(\frac{1}{200} \lambda\right)^{n}}=C(n)
$$

indices $l \in \mathcal{I}(q, t)$ with $\Xi_{l}(x)=m$ and $x \in 2 B_{l}$. Hence we deduce

$$
\sum_{\substack{i \in \mathcal{I}(q, t) \\ \Xi_{i}(x)=m}} \chi_{2 B_{i}}(x) \leq C(n)
$$

III. We have $i \in J(i)$ and so $\Xi_{i}(x) \neq 0$ for all $x \in 2 B_{i}$. Hence, with $x \in \mathbb{R}^{N}$, the term

$$
\chi_{2 B_{i}}(x) \Xi_{i}(x)^{-2}:= \begin{cases}\Xi_{i}(x)^{-2} & \text { if } x \in 2 B_{i} \\ 0 & \text { otherwise }\end{cases}
$$

is well-defined. Now there exists some constant $C(n)$ so that, for all $x \in \mathbb{R}^{N}$, we get

$$
\begin{aligned}
\sum_{i \in \mathcal{I}(q, t)} \chi_{2 B_{i}}(x) \Xi_{i}(x)^{-2} & =\sum_{m=1}^{\infty} \sum_{\substack{i \in \mathcal{I}(q, t) \\
\Xi_{i}(x)=m}} \chi_{2 B_{i}}(x) \Xi_{i}(x)^{-2} \\
& =\sum_{m=1}^{\infty} \frac{1}{m^{2}} \sum_{\substack{i \in \mathcal{I}(q, t) \\
\Xi_{i}(x)=m}} \chi_{2 B_{i}}(x) \leq C(n) .
\end{aligned}
$$


IV. Let $i \in \mathcal{I}(q, t)$. Since $i \in J(i)$, we have $\Xi_{i}(x) \neq 0$ for $x \in 2 B_{i}$. We obtain with Hölder's inequality

$$
\begin{aligned}
& \left(\frac{1}{\left(\operatorname{diam} R_{i}\right)^{n}} \int_{2 B_{i}} d\left(z, P^{(q, t, X)}\right)^{\frac{1}{3}} \Xi_{i}(z)^{\frac{-2}{3}} \Xi_{i}(z)^{\frac{2}{3}} \mathrm{~d} \mu(z)\right)^{3} \\
\leq & \left(\left(\frac{1}{\left(\operatorname{diam} R_{i}\right)^{n}} \int_{2 B_{i}}\left(d\left(z, P^{(q, t, X)}\right)^{\frac{1}{3}} \Xi_{i}(z)^{\frac{-2}{3}}\right)^{3} \mathrm{~d} \mu(z)\right)^{\frac{1}{3}}\right)^{3} \\
& \left(\left(\frac{1}{\left(\operatorname{diam} R_{i}\right)^{n}} \int_{2 B_{i}}\left(\Xi_{i}(z)^{\frac{2}{3}}\right)^{\frac{3}{2}} \mathrm{~d} \mu(z)\right)^{\frac{2}{3}}\right)^{3} \\
= & \frac{1}{\left(\operatorname{diam} R_{i}\right)^{n}} \int_{2 B_{i}} d\left(z, P^{(q, t, X)}\right) \Xi_{i}(z)^{-2} \mathrm{~d} \mu(z)\left(\frac{1}{\left(\operatorname{diam} R_{i}\right)^{n}} \int_{2 B_{i}} \Xi_{i}(z) \mathrm{d} \mu(z)\right)^{2} \\
\leq & C\left(n, C_{0}\right) \frac{1}{\left(\operatorname{diam} R_{i}\right)^{n}} \int_{2 B_{i}} d\left(z, P^{(q, t, X)}\right) \Xi_{i}(z)^{-2} \mathrm{~d} \mu(z) .
\end{aligned}
$$

V. We have

$$
\frac{1}{t^{n+1}} \int_{\bigcup_{i \in \mathcal{I}(q, t)} 2 B_{i}} d\left(z, P^{(q, t, X)}\right) \mathrm{d} \mu(z) \leq 2 \beta_{1 ; k}(X, t),
$$

where $X \in B(\tilde{X}(q), 200 t)$ (cf. page 106).

Proof. At first, we prove that there exists some constant $\hat{C}>1$ so that for $i \in \mathcal{I}(q, t)$

$$
2 B_{i} \subset B(X, \hat{C} t) .
$$

Let $i \in \mathcal{I}(q, t)$. By definition of $\mathcal{I}(q, t)$ (see page 106), we obtain $R_{i} \cap B(q, t) \neq \emptyset$. Let $\tilde{u} \in R_{i} \cap B(q, t)$. Since $\frac{D(q)}{100}<t$ (see page 106), we get, using the triangle inequality, $D(\tilde{u}) \leq D(q)+d(q, \tilde{u})<101 t$. It follows with Lemma 6.14 (i) that

$$
\operatorname{diam} R_{i} \leq \frac{1}{10} D(\tilde{u})<11 t .
$$

With Lemma 6.15 (ii) and (7.13) from page 105, we get $(X \in B(\tilde{X}, 200 t)$, see page 106)

$$
\begin{aligned}
d\left(\pi\left(B_{i}\right), \pi(X)\right) & \leq d\left(\pi\left(B_{i}\right), \tilde{u}\right)+d(\tilde{u}, q)+d(q, \pi(\tilde{X}))+d(\pi(\tilde{X}), \pi(X)) \\
& \stackrel{(7.13)}{\leq} d\left(\pi\left(B_{i}\right), R_{i}\right)+\operatorname{diam} R_{i}+t+200 t+d(\tilde{X}, X) \stackrel{(7.27)}{\leq} C t .
\end{aligned}
$$

Now let $x \in 2 B_{i}=B\left(X_{i}, 2 t_{i}\right)$. Since $\left(X_{i}, t_{i}\right) \in S$, we get, using Lemma 6.15 (i),

$$
d(x)=\inf _{(Y, s) \in S}(d(Y, x)+s) \leq d\left(X_{i}, x\right)+t_{i}<2 \operatorname{diam} B_{i} \leq 400 \operatorname{diam} R_{i} \stackrel{(7.27)}{<} 4400 t,
$$

and, because $X \in B(\tilde{X}, 200 t) \cap F$, we deduce

$$
d(X)=\inf _{(Y, s) \in S}(d(Y, X)+s) \stackrel{(7.13)}{\leq} d(\tilde{X}, X)+T \stackrel{(7.13)}{\leq} 400 t
$$


With Lemma 6.15 (i) and estimates (7.27) and (7.28), we obtain

$$
d(\pi(x), \pi(X)) \leq d\left(\pi(x), \pi\left(B_{i}\right)\right)+\operatorname{diam} B_{i}+d\left(\pi\left(B_{i}\right), \pi(X)\right) \leq C t .
$$

Now there exists some constant $\hat{C}>1$ so that, we get with Lemma 6.12

$$
d(x, X) \leq 6(d(x)+d(X))+2 d(\pi(x), \pi(X)) \leq \hat{C} t .
$$

All in all we have proven that, for all $i \in \mathcal{I}(q, t)$, we have

$$
2 B_{i} \subset B(X, \hat{C} t) .
$$

Since $k \geq \tilde{k} \geq \hat{C}$ (see page 103), we get with condition (7.14) from page 106

$$
\begin{aligned}
\frac{1}{t^{n+1}} \int_{\bigcup_{i \in \mathcal{I}(q, t)} 2 B_{i}} d\left(z, P^{(q, t, X)}\right) \mathrm{d} \mu(z) & \leq \frac{1}{t^{n}} \int_{B(X, k t)} \frac{d\left(z, P^{(q, t, X)}\right)}{t} \mathrm{~d} \mu(z) \\
& =\beta_{1 ; k}^{P^{(q, t, X)}}(X, t) \stackrel{(7.14)}{\leq} 2 \beta_{1 ; k}(X, t) .
\end{aligned}
$$

Now with the monotone convergence theorem [EG92, 1.3, Thm. 2] (used in the following to interchange the summation and the integration), we deduce

$$
\begin{aligned}
& \sum_{i \in \mathcal{I}(q, t)}\left(\frac{\operatorname{diam} R_{i}}{t}\right)^{n} \frac{1}{t}\left(\frac{1}{\left(\operatorname{diam} R_{i}\right)^{n}} \int_{2 B_{i}} d\left(z, P^{(q, t, X)}\right)^{\frac{1}{3}} \mathrm{~d} \mu(z)\right)^{3} \\
& \stackrel{\mathrm{IV}}{\leq} C\left(n, C_{0}\right) \sum_{i \in \mathcal{I}(q, t)}\left(\frac{\operatorname{diam} R_{i}}{t}\right)^{n} \frac{1}{t} \frac{1}{\left(\operatorname{diam} R_{i}\right)^{n}} \int_{2 B_{i}} d\left(z, P^{(q, t, X)}\right) \Xi_{i}(z)^{-2} \mathrm{~d} \mu(z) \\
&= \frac{C\left(n, C_{0}\right)}{t^{n+1}} \sum_{i \in \mathcal{I}(q, t)} \int_{2 B_{i}} d\left(z, P^{(q, t, X)}\right) \Xi_{i}(z)^{-2} \mathrm{~d} \mu(z) \\
& \stackrel{\mathrm{III}}{=} \frac{C\left(n, C_{0}\right)}{t^{n+1}} \sum_{i \in \mathcal{I}(q, t)} \int_{\bigcup_{l \in \mathcal{I}(q, t)} 2 B_{l}} d\left(z, P^{(q, t, X)}\right) \chi_{2 B_{i}}(z) \Xi_{i}(z)^{-2} \mathrm{~d} \mu(z) \\
&= \frac{C\left(n, C_{0}\right)}{t^{n+1}} \int_{\bigcup_{l \in \mathcal{I}(q, t)} 2 B_{l}} d\left(z, P^{(q, t, X)}\right) \sum_{i \in \mathcal{I}(q, t)} \chi_{2 B_{i}}(z) \Xi_{i}(z)^{-2} \mathrm{~d} \mu(z) \\
& \mathrm{III} \frac{C\left(n, C_{0}\right)}{t^{n+1}} \int_{\bigcup_{l \in \mathcal{I}(q, t)} 2 B_{l}} d\left(z, P^{(q, t, X)}\right) \mathrm{d} \mu(z) \\
& \leq C\left(n, C_{0}\right) \beta_{1 ; k}(X, t)
\end{aligned}
$$

and so Lemma 7.24 is proven.

Now we can give some estimate for $\gamma(q, t)$, where $q \in U_{10}$ and $\frac{D(q)}{100}<t<2$. Using the inequalities (7.15), (7.18), (7.19), (7.20), Lemma 7.23 and Lemma 7.24, we get using $T \leq 200 t$ 
(cf. Lemma 7.19) for every $X \in B(\tilde{X}, T) \cap F \subset B(\tilde{X}, 200 t) \cap F$ where $C=C\left(N, n, C_{0}\right)$

$$
\begin{aligned}
& \gamma(q, t) \stackrel{(7.15)}{\leq} K_{0}+\sum_{i \in \mathcal{I}(q, t)} K_{i} \\
& \stackrel{(7.18)}{\leq} C \beta_{1 ; k}(X, t)+\sum_{i \in \mathcal{I}(q, t)} K_{i} \\
& \stackrel{(7.19)}{\leq} C \beta_{1 ; k}(X, t)+\sum_{i \in \mathcal{I}(q, t)}\left[\frac{1}{t^{n}} \int_{B(q, t) \cap R_{i}} \frac{d\left(u+A(u), P_{i}\right)}{t} \mathrm{~d} \mathcal{H}^{n}(u)\right. \\
& \left.+\omega_{n}\left(\frac{\operatorname{diam} R_{i}}{t}\right)^{n} \sup \left\{\frac{d\left(w, P^{(q, t, X)}\right)}{t} \mid w \in P_{i}, d\left(w, B_{i}\right) \leq C_{4} \operatorname{diam} R_{i}\right\}\right] \\
& \stackrel{(7.20)}{\leq} C \beta_{1 ; k}(X, t)+\sum_{i \in \mathcal{I}(q, t)}\left[C \varepsilon\left(\frac{\operatorname{diam} R_{i}}{t}\right)^{n+1}\right. \\
& \left.+\omega_{n}\left(\frac{\operatorname{diam} R_{i}}{t}\right)^{n} \sup \left\{\frac{d\left(w, P^{(q, t, X)}\right)}{t} \mid w \in P_{i}, d\left(w, B_{i}\right) \leq C_{4} \operatorname{diam} R_{i}\right\}\right] \\
& \stackrel{\text { L. }}{\leq}{ }^{7.23} C \beta_{1 ; k}(X, t)+\sum_{i \in \mathcal{I}(q, t)}\left[C \varepsilon\left(\frac{\operatorname{diam} R_{i}}{t}\right)^{n+1}\right. \\
& \left.+\omega_{n}\left(\frac{\operatorname{diam} R_{i}}{t}\right)^{n}\left(C \varepsilon \frac{\operatorname{diam} R_{i}}{t}+C \frac{1}{t}\left(\frac{1}{\left(\operatorname{diam} R_{i}\right)^{n}} \int_{2 B_{i}} d\left(z, P^{(q, t, X)}\right)^{\frac{1}{3}} \mathrm{~d} \mu(z)\right)^{3}\right)\right] \\
& =C \beta_{1 ; k}(X, t)+C \varepsilon \sum_{i \in \mathcal{I}(q, t)}\left(\frac{\operatorname{diam} R_{i}}{t}\right)^{n+1} \\
& +C \sum_{i \in \mathcal{I}(q, t)}\left(\frac{\operatorname{diam} R_{i}}{t}\right)^{n} \frac{1}{t}\left(\frac{1}{\left(\operatorname{diam} R_{i}\right)^{n}} \int_{2 B_{i}} d\left(z, P^{(q, t, X)}\right)^{\frac{1}{3}} \mathrm{~d} \mu(z)\right)^{3}
\end{aligned}
$$

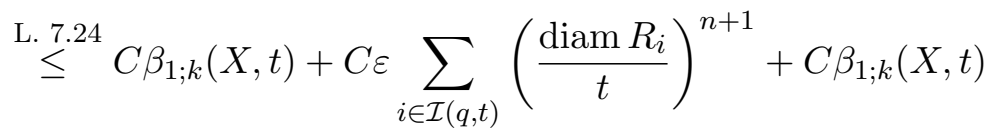

$$
\begin{aligned}
& \leq C\left(N, n, C_{0}\right) \beta_{1 ; k}(X, t)+C\left(N, n, C_{0}\right) \varepsilon \sum_{i \in \mathcal{I}(q, t)}\left(\frac{\operatorname{diam} R_{i}}{t}\right)^{n+1} .
\end{aligned}
$$

With Lemma 7.19, we get $(\tilde{X}, T) \in S \subset S_{\text {total }}$ and $20 t \leq T \leq 200 t$. Using this, the definition of $\delta=\delta(n)$ on page 65 and (B) from page 64 , we get

$$
\begin{aligned}
& \gamma(q, t)^{p} \\
\leq & \frac{2}{\delta} \frac{\mu(B(\tilde{X}, T))}{T^{n}} \gamma(q, t)^{p} \\
= & \frac{2}{\delta T^{n}} \int_{B(\tilde{X}, T)} \gamma(q, t)^{p} \mathrm{~d} \mu(X)
\end{aligned}
$$




$$
\begin{aligned}
\leq & \frac{2}{\delta(20 t)^{n}} \int_{B(\tilde{X}, 200 t)}\left[C \beta_{1 ; k}(X, t)+C \varepsilon \sum_{i \in \mathcal{I}(q, t)}\left(\frac{\operatorname{diam} R_{i}}{t}\right)^{n+1}\right]^{p} \mathrm{~d} \mu(X) \\
\stackrel{\text { (B) }}{\leq} & \frac{C}{t^{n}} \int_{B(\tilde{X}, 200 t)} 2^{p-1} \beta_{1 ; k}(X, t)^{p} \mathrm{~d} \mu(X) \\
& +2^{p-1} \frac{2 C_{0}(400 t)^{n}}{\delta(20 t)^{n}}\left(C \varepsilon \sum_{i \in \mathcal{I}(q, t)}\left(\frac{\operatorname{diam} R_{i}}{t}\right)^{n+1}\right)^{p} \\
\leq & C \frac{1}{t^{n}} \int_{B(\tilde{X}, 200 t)} \beta_{1 ; k}(X, t)^{p} \mathrm{~d} \mu(X)+C \varepsilon^{p}\left(\sum_{i \in \mathcal{I}(q, t)}\left(\frac{\operatorname{diam} R_{i}}{t}\right)^{n+1}\right)^{p},
\end{aligned}
$$

where $C=C\left(N, n, p, C_{0}\right)$. We recall that for every $q \in U_{10}$ there exists some $\tilde{X}=\tilde{X}(q)$ (cf. Lemma 7.19) such that the previous inequality is valid. This implies

$$
\begin{aligned}
& \int_{U_{10}} \int_{\frac{D(q)}{100}}^{2} \gamma(q, t)^{p} \frac{\mathrm{d} t}{t} \mathrm{~d} \mathcal{H}^{n}(q) \\
\leq & 2^{\frac{p}{2}-1} C \underbrace{\int_{U_{10}} \int_{\frac{D(q)}{100}}^{2} \frac{1}{t^{n}} \int_{B(\tilde{X}(q), 200 t)} \beta_{1 ; k}(X, t)^{p} \mathrm{~d} \mu(X) \frac{\mathrm{d} t}{t} \mathrm{~d} \mathcal{H}^{n}(q)}_{=: a} \\
+ & 2^{\frac{p}{2}-1} C \varepsilon^{p} \underbrace{\int_{U_{10}} \int_{\frac{D(q)}{100}}^{2}\left(\sum_{i \in \mathcal{I}(q, t)}\left(\frac{\operatorname{diam} R_{i}}{t}\right)^{n+1}\right)^{p} \frac{\mathrm{d} t}{t} \mathrm{~d} \mathcal{H}^{n}(q)}_{=: b} \\
\leq & C\left(N, n, p, C_{0}\right) a+C\left(N, n, p, C_{0}\right) \varepsilon^{p} b .
\end{aligned}
$$

To estimate $a$ and $b$, we need the following lemma.

Lemma 7.25. Let $q \in U_{10}, \frac{D(q)}{100} \leq t \leq 2$ and $X \in B(\tilde{X}(q), 200 t) \cap F$, where $\tilde{X}(q)$ is given by Lemma 7.19 on page 105 . Then

$$
d(\pi(X), q) \leq 400 t
$$

and there exists some $\tilde{\lambda}=\tilde{\lambda}\left(N, n, C_{0}\right)>0$ so that, with $k_{0}=401$, we have

$$
\tilde{\delta}_{k_{0}}(B(X, t))=\sup _{y \in B\left(X, k_{0} t\right)} \frac{\mu(B(y, t))}{t^{n}} \geq \tilde{\lambda}
$$

where $\tilde{\delta}_{k_{0}}(B(X, t))$ was defined on page 45. Furthermore, there holds for all $i \in \mathcal{I}(q, t)$ that

$$
d\left(q, R_{i}\right) \leq t, \quad \operatorname{diam} R_{i}<11 t,
$$

and there exists some constant $C=C(n)$ with

$$
\begin{aligned}
\sum_{i \in \mathcal{I}(q, t)}\left(\frac{\operatorname{diam} R_{i}}{t}\right)^{n+1} & \leq C, \\
\sum_{i \in I_{12}}\left(\operatorname{diam} R_{i}\right)^{n} & \leq C .
\end{aligned}
$$


Proof. Let $q \in U_{10}, \frac{D(q)}{100} \leq t \leq 2$ and $X \in B(\tilde{X}(q), 200 t) \cap F$. We have $d(X, \tilde{X}(q)) \leq 200 t$ and, with (7.13), we get $d(\pi(\tilde{X}(q)), q) \leq 200 t$. This implies

$$
d(\pi(X), q) \leq d(\pi(X), \pi(\tilde{X}(q)))+d(\pi(\tilde{X}(q)), q) \leq d(X, \tilde{X}(q))+200 t \leq 400 t .
$$

With (7.13), we obtain $(\tilde{X}(q), T) \in S \subset S_{\text {total }}$ and, by definition of $S_{\text {total }}$, we conclude $\frac{\mu(B(\tilde{X}(q), T))}{T^{n}} \geq \frac{\delta}{2}$. We have $B(\tilde{X}(q), T) \subset B(X, 400 t)$ and hence

$$
\delta(B(X, 400 t))=\frac{\mu(B(X, 400 t))}{(400 t)^{n}} \stackrel{(7.13)}{\geq} \frac{1}{20^{n}} \frac{\mu(B(\tilde{X}(q), T))}{T^{n}} \geq \frac{\delta}{2 \cdot 20^{n}} .
$$

Applying Corollary 5.3 (ii) with $\lambda=\frac{\delta}{2 \cdot 20^{n}}$ on $B(X, 400 t)$, we get constants $C_{1}=C_{1}\left(N, n, C_{0}\right)$, $C_{2}=C_{2}\left(N, n, C_{0}\right)$ and in particular one ball $B(x, s)$ with $s=\frac{400 t}{C_{1}}$ and

$$
\mu(B(x, s) \cap B(X, 400 t)) \geq \frac{(400 t)^{n}}{C_{2}} .
$$

We have defined $\delta \leq \frac{2}{50^{n}}$ (cf. (6.2) on page 65), and Lemma 5.2 gives us $C_{1}=\frac{4 \cdot 120^{n} n^{n+1} N_{0} C_{0}}{\frac{\delta}{2 \cdot 20^{n}}}>$ 400. That yields $s<t$. From (7.35), we get $B(x, s) \cap B(X, 400 t) \neq \emptyset$ which implies $d(x, X) \leq$ $400 t+s<401 t$ and

$$
\sup _{y \in B(X, 401 t)} \frac{\mu(B(y, t))}{t^{n}} \geq \frac{\mu(B(x, s))}{t^{n}} \stackrel{(7.35)}{\geq} \frac{1}{t^{n}} \frac{(400 t)^{n}}{C_{2}}=\frac{400^{n}}{C_{2}}=: \tilde{\lambda} .
$$

Let $i \in \mathcal{I}(q, t)$. Due to the definition of $\mathcal{I}(q, t)$ (see page 106), we have

$$
d\left(q, R_{i}\right) \leq t
$$

and we can choose some $\tilde{u} \in R_{i} \cap B(q, t)$. With Lemma 6.14 (i), we obtain

$$
\operatorname{diam} R_{i} \leq \frac{1}{10} D(\tilde{u}) \leq \frac{1}{10}(D(q)+d(q, \tilde{u}))<11 t .
$$

The intervals $R_{i}$ have disjoint interior (see Lemma 6.14 (ii)) and, from $R_{i} \cap B(q, t) \neq \emptyset$ for all $i \in \mathcal{I}(q, t)$, we get $R_{i} \subset B(q, 12 t)$. With Lemma A.7, this implies

$$
\begin{aligned}
\sum_{i \in \mathcal{I}(q, t)}\left(\frac{\operatorname{diam} R_{i}}{t}\right)^{n+1} \stackrel{(7.32)}{<} 11 t \frac{1}{t^{n+1}} \sum_{i \in \mathcal{I}(q, t)}\left(\operatorname{diam} R_{i}\right)^{n} \\
=\frac{11}{t^{n}} \sum_{i \in \mathcal{I}(q, t)}(\sqrt{n})^{n} \mathcal{H}^{n}\left(R_{i}\right) \\
=\frac{11}{t^{n}}(\sqrt{n})^{n} \mathcal{H}^{n}(B(q, 12 t)) \\
=\frac{11}{t^{n}}(\sqrt{n})^{n}(12 t)^{n} \omega_{n}=11 \cdot 12^{n}(\sqrt{n})^{n} \omega_{n}=C(n) .
\end{aligned}
$$


Now let $i \in I_{12}$. We have $R_{i} \cap B(0,12) \neq \emptyset$. If $(Y, r) \in S \subset S_{\text {total }}$, we get $Y \in F \subset B(0,5)$ (cf. (A) on page 64) and hence we obtain $d(\pi(Y), 0) \leq 5$ as well as $r \leq 50$. With $\tilde{v} \in R_{i} \cap B(0,12)$ and Lemma 6.14 (i), we get

$$
\operatorname{diam} R_{i} \leq \frac{1}{10} D(\tilde{v})=\frac{1}{10} \inf _{(Y, r) \in S}(d(\pi(Y), \tilde{v})+r) \leq \frac{1}{10}(5+12+50)<7 .
$$

Hence, for all $i \in I_{12}$, we have $R_{i} \subset B(0,19)$ and the cubes $R_{i}$ have disjoint interior (cf. Lemma 6.14 (ii)). With Lemma A.7, we deduce

$$
\sum_{i \in I_{12}}\left(\operatorname{diam} R_{i}\right)^{n}=(\sqrt{n})^{n} \sum_{i \in I_{12}} \mathcal{H}^{n}\left(R_{i}\right) \leq(\sqrt{n})^{n} \mathcal{H}^{n}(B(0,19))=(19 \sqrt{n})^{n} \omega_{n}=C(n) .
$$

To control the terms $a$ and $b$ we will use Fubini's Theorem [EG92, 1.4, Thm. 1], in the following abbreviated by (F). Now, using Lemma 7.25 and Corollary $5.8\left(\lambda=\tilde{\lambda}, k_{0}=401\right)$, we conclude

$$
\begin{aligned}
a & =\int_{U_{10}} \int_{\frac{D(q)}{100}}^{2} \frac{1}{t^{n}} \int_{B(\tilde{X}(q), 200 t)} \beta_{1 ; k}(X, t)^{p} \mathrm{~d} \mu(X) \frac{\mathrm{d} t}{t} \mathrm{~d} \mathcal{H}^{n}(q) \\
& \leq \int_{U_{10}} \int_{0}^{2} \frac{1}{t^{n}} \int_{F} \mathbb{1}_{\left\{d(\pi(X), q) \leq 400 t, \tilde{\delta}_{k_{0}}(B(X, t)) \geq \tilde{\lambda}\right\}} \beta_{1 ; k}(X, t)^{p} \mathrm{~d} \mu(X) \frac{\mathrm{d} t}{t} \mathrm{~d} \mathcal{H}^{n}(q) \\
& \stackrel{(F)}{=} \int_{F} \int_{0}^{2} \frac{1}{t^{n}} \underbrace{\int_{U_{10}} \mathbb{1}_{\{d(\pi(X), q) \leq 400 t\}} \mathrm{d} \mathcal{H}^{n}(q)}_{=\mathcal{H}^{n}\left(B(\pi(X), 400 t) \cap U_{10}\right)} \mathbb{1}_{\left\{\tilde{\delta}_{k_{0}}(B(X, t)) \geq \tilde{\lambda}\right\}} \beta_{1 ; k}(X, t)^{p} \frac{\mathrm{d} t}{t} \mathrm{~d} \mu(X) \\
& =\omega_{n} 400^{n} \int_{F} \int_{0}^{2} \mathbb{1}_{\left\{\tilde{\delta}_{k_{0}}(B(X, t)) \geq \tilde{\lambda}\right\}} \beta_{1 ; k}(X, t)^{p} \frac{\mathrm{d} t}{t} \mathrm{~d} \mu(X) \\
& \leq C\left(N, n, \mathcal{K}, p, C_{0}, k\right) \mathcal{M}_{\mathcal{K}^{p}(\mu) .}
\end{aligned}
$$

Now we consider the integral $b$. We get using Fatou's Lemma [EG92, 1.3, Thm.1] to interchange the summation with the integration

$$
\begin{aligned}
& b=\int_{U_{10}} \int_{\frac{D(q)}{100}}^{2}\left(\sum_{i \in \mathcal{I}(q, t)}\left(\frac{\operatorname{diam} R_{i}}{t}\right)^{n+1}\right)^{p} \frac{\mathrm{d} t}{t} \mathrm{~d} \mathcal{H}^{n}(q) \\
& \stackrel{(7.33)}{\leq} C \int_{U_{10}} \int_{\frac{D(q)}{100}}^{2} \sum_{i \in \mathcal{I}(q, t)}\left(\frac{\operatorname{diam} R_{i}}{t}\right)^{n+1} \frac{\mathrm{d} t}{t} \mathrm{~d} \mathcal{H}^{n}(q) \\
& \stackrel{(7.32)}{\leq} C \int_{U_{10}} \int_{\frac{D(q)}{100}}^{2} \sum_{i \in I_{12}} \mathbb{1}_{\left\{t>\frac{\operatorname{diam} R_{i}}{11}, d\left(q, R_{i}\right) \leq t\right\}}\left(\frac{\operatorname{diam} R_{i}}{t}\right)^{n+1} \frac{\mathrm{d} t}{t} \mathrm{~d} \mathcal{H}^{n}(q) \\
& \stackrel{(F)}{\leq} C \sum_{i \in I_{12}}\left(\operatorname{diam} R_{i}\right)^{n+1} \int_{\frac{\operatorname{diam} R_{i}}{11}}^{\int_{U_{10}}^{\mathbb{1}_{\left\{d\left(q, R_{i}\right) \leq t\right\}} \mathrm{d} \mathcal{H}^{n}(q)}} \underbrace{\frac{\mathrm{d} t}{t^{n+2}}}_{\leq \omega_{n}\left(\frac{\operatorname{diam} R_{i}}{2}+t\right)^{n} \leq \omega_{n}(7 t)^{n}}
\end{aligned}
$$




$$
\begin{aligned}
& \leq C \sum_{i \in I_{12}}\left(\operatorname{diam} R_{i}\right)^{n+1} \int_{\frac{\operatorname{diam} R_{i}}{11}}^{\infty} \frac{1}{t^{2}} \mathrm{~d} t \\
& =C \sum_{i \in I_{12}}\left(\operatorname{diam} R_{i}\right)^{n} \\
& \stackrel{(7.34)}{\leq} C(n, p) .
\end{aligned}
$$

All in all, we obtain with the decomposition (7.9), Lemma 7.18, (7.12) and with (C) from page 64

$$
\begin{aligned}
\int_{U_{10}} \int_{0}^{2} \gamma(q, t)^{p} \frac{\mathrm{d} t}{t} \mathrm{~d} \mathcal{H}^{n}(q) & \leq C \varepsilon^{p}+\int_{U_{10}} \int_{\frac{D(q)}{100}}^{2} \gamma(q, t)^{p} \frac{\mathrm{d} t}{t} \mathrm{~d} \mathcal{H}^{n}(q) \\
& \leq C \varepsilon^{p}+C a+C \varepsilon^{p} b \\
& \leq C \varepsilon^{p}+C \mathcal{M}_{\mathcal{K}^{p}}(\mu)+C \varepsilon^{p}=C\left(N, n, \mathcal{K}, p, C_{0}, k\right) \varepsilon^{p}
\end{aligned}
$$

(C)

because $\mathcal{M}_{\mathcal{K}^{p}}(\mu) \leq \eta<\varepsilon^{p}$ (see page 64 and page 103).

This completes the proof of Theorem 7.17. 



\section{$8 \mathcal{Z}$ Is not too Small}

Our aim is to prove Theorem 6.1. In Definition 6.4, we defined a partition of the support $F$ of our measure $\mu$ in four parts, namely $\mathcal{Z}, F_{1}, F_{2}, F_{3}$. Then, in section 6.4 , we constructed some function $A$, the graph $\Gamma$ of which covers the set $\mathcal{Z}$. To get our main result, we need to know that we covered a major part of $F$. In this last part of the proof of Theorem 6.1 , we show that the $\mu$-measure of $F_{1}, F_{2}, F_{3}$ is quite small. In particular, we deduce $\mu\left(F_{1} \cup F_{2} \cup F_{3}\right) \leq \frac{1}{100}$. As stated at the beginning of section 6.1 on page 64 , this completes the proof of Theorem 6.1.

\subsection{Most of $F$ is close to the graph of $A$}

With

$$
K:=2(104 \cdot 10 \cdot 6+214)
$$

we define the set

$$
\begin{aligned}
G:= & \left\{x \in F \backslash \mathcal{Z} \mid \forall i \in I_{12} \text { with } \pi(x) \in 3 R_{i}, \text { we have } x \notin K B_{i}\right\} \\
& \cup\{x \in F \backslash \mathcal{Z} \mid \pi(x) \in \pi(\mathcal{Z})\} .
\end{aligned}
$$

At first, we show that the $\mu$-measure of $G$ is small.

Lemma 8.1. Let $0<\alpha \leq \frac{1}{280}$. There exist some $\tilde{\varepsilon}=\tilde{\varepsilon}\left(N, n, C_{0}, \alpha\right)$ so that, if $\eta<2 \tilde{\varepsilon}$ and $k \geq 4$, there exists some constant $C=C\left(N, n, \mathcal{K}, p, C_{0}\right)$ so that, for all $\varepsilon \in\left[\frac{\eta}{2}, \tilde{\varepsilon}\right)$, we have

$$
\mu(G) \leq C \mathcal{M}_{\mathcal{K}^{p}}(\mu) \stackrel{(C)}{\leq} C \eta,
$$

where the condition $(C)$ was given on page 64 .

Proof. Let $0<\alpha \leq \frac{1}{280}$ and $\tilde{\varepsilon}:=\min \left\{\bar{\varepsilon}, \frac{\alpha}{C}\right\}$ where $\bar{\varepsilon}$ is given by Lemma 6.12 and $\bar{C}=$ $\bar{C}\left(N, n, C_{0}\right)$ is a fixed constant defined in this proof on page 128. Furthermore let $\eta<2 \tilde{\varepsilon}$, $k \geq 4$ and $\eta \leq 2 \varepsilon<2 \tilde{\varepsilon}$.

Let $x \in G$. If $x \in G \backslash \pi^{-1}(\pi(\mathcal{Z})) \subset F \subset B(0,5)$, with Lemma 6.14 (ii), there exists some $i \in I_{12}$ with $\pi(x) \in R_{i} \subset 2 R_{i}$. Let $X_{i}$ be the centre of $B_{i}$ (cf. Lemma 6.15). We set

$$
X(x):= \begin{cases}X_{i} & \text { if } x \in G \backslash \pi^{-1}(\pi(\mathcal{Z})) \\ \pi(x)+A(\pi(x)) & \text { if } x \in G \cap \pi^{-1}(\pi(\mathcal{Z}))\end{cases}
$$


Claim 1: For all $x \in G$ and $X=X(x)$ defined as above, we have

$$
\begin{aligned}
d(x, X) & <7 d(x) \\
d(\pi(x), \pi(X)) & \leq \frac{d(x)}{10} \\
\frac{d(x)}{2} & \leq d(X, x) \\
\left(X, \frac{d(x)}{10}\right) & \in S .
\end{aligned}
$$

Proof of Claim 1.

1. Case: $x \in G \backslash \pi^{-1}(\pi(\mathcal{Z}))$.

Due to the definition of $G$ and $\pi(x) \in 2 R_{i} \subset 3 R_{i}$, we have $x \notin K B_{i}$. It follows with Lemma 6.15 (i), (ii) and $q \in R_{i}$

$$
\begin{aligned}
d\left(\pi(x), \pi\left(X_{i}\right)\right) & \leq d(\pi(x), q)+d\left(q, \pi\left(X_{i}\right)\right) \\
& \leq 2 \operatorname{diam} R_{i}+d\left(R_{i}, \pi\left(B_{i}\right)\right)+\frac{\operatorname{diam} B_{i}}{2}+\operatorname{diam} R_{i} \\
& \leq 3 \operatorname{diam} R_{i}+100 \operatorname{diam} R_{i}+\operatorname{diam} B_{i} \\
& \leq 104 \operatorname{diam} B_{i}
\end{aligned}
$$

With Lemma 6.15 , we know $\left(X_{i}, \frac{\operatorname{diam} B_{i}}{2}\right) \in S$ and hence we get

$$
d\left(X_{i}\right)=\inf _{(Y, t) \in S}\left(d\left(Y, X_{i}\right)+t\right) \leq d\left(X_{i}, X_{i}\right)+\frac{\operatorname{diam} B_{i}}{2}<\operatorname{diam} B_{i} .
$$

Since $x \notin K B_{i}$, we get with Lemma 6.12

$$
K \cdot \frac{\operatorname{diam} B_{i}}{2}<d\left(x, X_{i}\right) \leq 6\left(d(x)+d\left(X_{i}\right)\right)+2 d\left(\pi(x), \pi\left(X_{i}\right)\right)<6 d(x)+214 \operatorname{diam} B_{i}
$$

which yields by definition of $K(8.1)$

$$
104 \operatorname{diam} B_{i}<\frac{d(x)}{10} \text {. }
$$

From the previous two estimates, we get $d\left(x, X_{i}\right)<7 d(x)$, i.e., (8.2) holds in this case. Furthermore, we have (8.3) since

$$
d\left(\pi(x), \pi\left(X_{i}\right)\right) \stackrel{(8.6)}{\leq} 104 \operatorname{diam} B_{i} \stackrel{(8.8)}{<} \frac{d(x)}{10} .
$$

We have $\left(X_{i}, \frac{\operatorname{diam} B_{i}}{2}\right) \in S$, so we get

$$
d(x) \leq d\left(X_{i}, x\right)+\frac{\operatorname{diam} B_{i}}{2} \stackrel{(8.8)}{<} d\left(X_{i}, x\right)+\frac{d(x)}{2},
$$

and hence (8.4) holds in this case. Due to Lemma 6.10, we have $\frac{\operatorname{diam} B_{i}}{2} \stackrel{(8.8)}{<} \frac{d(x)}{10}<\frac{60}{10}<50$ so that with Lemma 6.3 (v) we deduce (8.5). 
2. Case: $x \in G \cap \pi^{-1}(\pi(\mathcal{Z}))$.

We have $\pi(x) \in \pi(\mathcal{Z})$ and hence $X=\pi(x)+A(\pi(x)) \in \mathcal{Z}$ (cf. Definition 6.21). By definition of $\mathcal{Z}$ and Lemma 6.3 (iv), we obtain $(X, \sigma) \in S$ for all $\sigma \in(0,50)$ and hence

$$
\frac{d(x)}{2} \leq d(x)=\inf _{(Y, s) \in S}(d(Y, x)+s) \leq d(X, x)+\sigma \stackrel{\sigma \rightarrow 0}{\longrightarrow} d(X, x),
$$

which establishes (8.4). Moreover, we have $d(\pi(X), \pi(x))=d(\pi(x), \pi(x))=0$. Using Lemma 6.11 , we obtain $d(X)=0$ and hence we get with Lemma $6.12 d(x, X) \leq 6(d(x)+d(X))+$ $2(d(\pi(x), \pi(X)))=6 d(x)$. Furthermore, we have with Lemma 6.10 that $\frac{d(x)}{10} \leq 6<50$ so that by definition of $\mathcal{Z}$, we get $\left(X, \frac{d(x)}{10}\right) \in S$. End of Proof of Claim 1 .

Let $P_{\left(X, \frac{d(x)}{10}\right)}$ be the plane associated to $B\left(X, \frac{d(x)}{10}\right)$ (cf. Lemma $6.3\left(\right.$ vi)). Since $S \subset S_{\text {total }}$, we obtain

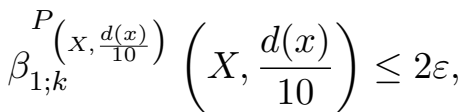

$$
\begin{aligned}
& \delta\left(B\left(X, \frac{d(x)}{10}\right)\right) \geq \frac{1}{2} \delta
\end{aligned}
$$

and

$$
\Varangle\left(P_{\left(X, \frac{d(x)}{10}\right)}, P_{0}\right) \leq \alpha .
$$

We define the set

$$
\Upsilon:=\left\{u \in B\left(X, \frac{d(x)}{10}\right) \mid d\left(u, P_{\left(X, \frac{d(x)}{10}\right)}\right) \leq \frac{8}{\delta} \frac{d(x)}{10} \varepsilon\right\} .
$$

We get

$$
\begin{aligned}
\mu\left(B\left(X, \frac{d(x)}{10}\right) \backslash \Upsilon\right) & =\frac{1}{\frac{8}{\delta} \frac{d(x)}{10} \varepsilon} \int_{B\left(X, \frac{d(x)}{10}\right) \backslash \Upsilon} \frac{8}{\delta} \frac{d(x)}{10} \varepsilon \mathrm{d} \mu(u) \\
& <\frac{1}{\frac{8}{\delta} \frac{d(x)}{10} \varepsilon} \int_{B\left(X, \frac{d(x)}{10}\right) \backslash \Upsilon} d\left(u, P\left(X, \frac{d(x)}{10}\right) \mathrm{d} \mu(u)\right. \\
& \leq \frac{\delta}{8 \varepsilon}\left(\frac{d(x)}{10}\right)^{n} \frac{1}{\left(\frac{d(x)}{10}\right)^{n}} \int_{B\left(X, k \frac{d(x)}{10}\right)} \frac{d\left(u, P\left(X, \frac{d(x)}{10}\right)\right.}{\frac{d(x)}{10}} \mathrm{~d} \mu(u) \\
& =\frac{\delta}{8 \varepsilon}\left(\frac{d(x)}{10}\right)^{n} \beta_{1 ; k}\left(x, \frac{d(x)}{10}\right) \\
& \left(X, \frac{d(x)}{10}\right) \\
& \leq \frac{\delta}{8 \varepsilon}\left(\frac{d(x)}{10}\right)^{n} 2 \varepsilon \\
& \leq \frac{\delta}{4}\left(\frac{d(x)}{10}\right)^{n}
\end{aligned}
$$


and hence, because $\Upsilon \subset B\left(X, \frac{d(x)}{10}\right)$,

$$
\begin{aligned}
\mu\left(B\left(X, \frac{d(x)}{10}\right) \cap \Upsilon\right)=\mu(\Upsilon) & \geq \mu\left(B\left(X, \frac{d(x)}{10}\right)\right)-\mu\left(B\left(X, \frac{d(x)}{10}\right) \backslash \Upsilon\right) \\
& \stackrel{(8.10)}{\geq} \frac{\delta}{2}\left(\frac{d(x)}{10}\right)^{n}-\frac{\delta}{4}\left(\frac{d(x)}{10}\right)^{n} \\
& =\frac{\delta}{4}\left(\frac{d(x)}{10}\right)^{n} .
\end{aligned}
$$

With Corollary $5.3\left(\lambda=\frac{\delta}{4}, t=\frac{d(x)}{10}\right)$, there exist constants $C_{1}=C_{1}\left(N, n, C_{0}\right), C_{2}=$ $C_{2}\left(N, n, C_{0}\right)$ and an $\left(n, 10 n \frac{d(x)}{10 C_{1}}\right)$-simplex

$$
T=\Delta\left(x_{0}, \ldots, x_{n}\right) \in F \cap B\left(X, \frac{d(x)}{10}\right) \cap \Upsilon
$$

so that for all $j \in\{0, \ldots, n\}$

$$
\mu\left(B\left(x_{j}, \frac{d(x)}{10 C_{1}}\right) \cap B\left(X, \frac{d(x)}{10}\right) \cap \Upsilon\right) \geq\left(\frac{d(x)}{10}\right)^{n} \frac{1}{C_{2}}
$$

Let $y_{j} \in B\left(x_{j}, \frac{d(x)}{10 C_{1}}\right) \cap \Upsilon$ for all $j \in\{0, \ldots, n\}$. By applying Lemma $2.14 n+1$ times, we find that $\Delta\left(y_{0}, \ldots, y_{n}\right)$ is an $\left(n, 8 n \frac{d(x)}{10 C_{1}}\right)$-simplex.

Claim 2: For all $x \in G$, we have

$$
d\left(x, \operatorname{aff}\left(y_{0}, \ldots, y_{n}\right)\right) \geq \frac{d(x)}{4}
$$

Proof of Claim 2. Let $P_{y}:=\operatorname{aff}\left(y_{0}, \ldots, y_{n}\right)$ be the plane through $y_{0}, \ldots, y_{n}$. Applying Lemma $2.28\left(C=\frac{C_{1}}{8 n}, \hat{C}=1, t=\frac{d(x)}{10}, \sigma=\frac{8}{\delta} \varepsilon, P_{1}=P_{y}, P_{2}=P_{\left(X, \frac{d(x)}{10}\right)}, S=\Delta\left(y_{0}, \ldots, y_{n}\right), x=X\right.$, $m=n)$ yields

$$
\Varangle\left(P_{y}, P_{\left(X, \frac{d(x)}{10}\right)}\right) \leq \underbrace{4 n\left(10^{n}+1\right) \frac{1}{2}\left(\frac{C_{1}}{4}\right)^{n+1} \frac{8}{\delta}}_{=: \bar{C}} \varepsilon \leq \alpha,
$$

where we use that $\varepsilon \leq \tilde{\varepsilon} \leq \frac{\alpha}{\bar{C}}$. So, with (8.11) and Lemma 2.20, we obtain

$$
\Varangle\left(P_{y}, P_{0}\right) \leq \Varangle\left(P_{y}, P_{\left(X, \frac{d(x)}{10}\right)}\right)+\Varangle\left(P_{\left(X, \frac{d(x)}{10}\right)}, P_{0}\right) \leq 2 \alpha .
$$

Let $\hat{P}_{y} \in \mathcal{P}(N, n)$ be the $n$-dimensional plane parallel to $P_{y}$ with $X \in \hat{P}_{y}$, and $\hat{P}_{0} \in \mathcal{P}(N, n)$ 
be the plane parallel to $P_{0}$ with $X \in \hat{P}_{0}$. We have $\alpha \leq \frac{1}{280}$ and hence

$$
\begin{aligned}
d\left(\pi_{\hat{P}_{y}}(x), \pi_{\hat{P}_{0}}(x)\right) & =\left|\pi_{\hat{P}_{y}-X}(x-X)-\pi_{\hat{P}_{0}-X}(x-X)\right| \\
& =|x-X|\left|\pi_{\hat{P}_{y}-X}\left(\frac{x-X}{|x-X|}\right)-\pi_{\hat{P}_{0}-X}\left(\frac{x-X}{|x-X|}\right)\right| \\
& \leq d(x, X) \Varangle\left(\hat{P}_{y}-X, \hat{P}_{0}-X\right) \\
& \stackrel{(8.2)}{<} 7 d(x) \Varangle\left(P_{y}, P_{0}\right) \\
& \stackrel{(8.15)}{\leq} \frac{d(x)}{20},
\end{aligned}
$$

and using (8.3) we get

$$
d\left(\pi_{\hat{P}_{0}}(x), X\right)=d\left(\pi_{\hat{P}_{0}}(x), \pi_{\hat{P}_{0}}(X)\right)=d(\pi(x), \pi(X)) \leq \frac{d(x)}{10},
$$

which implies

$$
d\left(\pi_{\hat{P}_{y}}(x), X\right) \leq d\left(\pi_{\hat{P}_{y}}(x), \pi_{\hat{P}_{0}}(x)\right)+d\left(\pi_{\hat{P}_{0}}(x), X\right) \leq \frac{d(x)}{20}+\frac{d(x)}{10} .
$$

From this, we obtain using $y_{0} \in \Upsilon \subset B\left(X, \frac{d(x)}{10}\right)$ and $d\left(P_{y}, \hat{P}_{y}\right)=d\left(X, P_{y}\right) \leq d\left(X, y_{0}\right) \leq \frac{d(x)}{10}$

$$
\begin{aligned}
\frac{d(x)}{2} & \stackrel{(8.4)}{\leq} d(x, X) \\
& \leq d\left(x, \hat{P}_{y}\right)+d\left(\pi_{\hat{P}_{y}}(x), X\right) \\
& \leq d\left(x, P_{y}\right)+d\left(P_{y}, \hat{P}_{y}\right)+\frac{3 d(x)}{20} \\
& \leq d\left(x, P_{y}\right)+\frac{d(x)}{4}
\end{aligned}
$$

and gain $d\left(x, P_{y}\right) \geq \frac{d(x)}{4}$. End of Proof of Claim 2.

With (8.2) and

$$
d\left(y_{j}, X\right) \leq d\left(y_{j}, x_{j}\right)+d\left(x_{j}, X\right) \leq \frac{d(x)}{10 C_{1}}+\frac{d(x)}{10},
$$

we obtain $y_{0}, \ldots y_{n}, x \in B(X, 7 d(x)) \subset B\left(X, \frac{C_{1}}{8 n} \frac{d(x)}{10}\right)$, where we used the explicit characterisation of $C_{1}$ given in Lemma 5.2. Hence we get with the second property of a $\mu$-proper integrand (see Definition 4.4) the existence of some $\tilde{C}=\tilde{C}\left(N, n, \mathcal{K}, p, C_{0}\right) \geq 1$ with

$$
\mathcal{K}^{p}\left(y_{0}, \ldots, y_{n}, x\right) \geq \frac{1}{\left(\frac{d(x)}{10}\right)^{n(n+1)} \tilde{C}} \underbrace{\left(\frac{d\left(x, \operatorname{aff}\left(y_{0}, \ldots, y_{n}\right)\right)}{10}\right)^{p}}_{\substack{(8.14) \\ \geq\left(\frac{5}{2}\right)^{p}>1}} \tilde{C}^{-1}\left(\frac{10}{d(x)}\right)^{n(n+1)} .
$$


This estimate holds for all $y_{i} \in B\left(x_{i}, \frac{d(x)}{10 C_{1}}\right) \cap \Upsilon$. It follows

$$
\begin{aligned}
& \int \ldots \int \mathcal{K}^{p}\left(y_{0}, \ldots, y_{n}, x\right) \mathrm{d} \mu\left(y_{0}\right) \ldots \mathrm{d} \mu\left(y_{n}\right) \\
\geq & \int_{B\left(x_{n}, \frac{d(x)}{10 C_{1}}\right) \cap \Upsilon} \ldots \int_{B\left(x_{0}, \frac{d(x)}{10 C_{1}}\right) \cap \Upsilon} \mathcal{K}^{p}\left(y_{0}, \ldots, y_{n}, x\right) \mathrm{d} \mu\left(y_{0}\right) \ldots \mathrm{d} \mu\left(y_{n}\right) \\
\geq & \tilde{C}^{-1}\left(\frac{10}{d(x)}\right)^{n(n+1)} \int_{B\left(x_{n}, \frac{d(x)}{10 C_{1}}\right) \cap \Upsilon} \ldots \int_{B\left(x_{0}, \frac{d(x)}{10 C_{1}}\right) \cap \Upsilon} \mathrm{d} \mu\left(y_{0}\right) \ldots \mathrm{d} \mu\left(y_{n}\right) \\
= & \tilde{C}^{-1}\left(\frac{10}{d(x)}\right)^{n(n+1)} \mu\left(B\left(x_{0}, \frac{d(x)}{10 C_{1}}\right) \cap \Upsilon\right) \ldots \mu\left(B\left(x_{n}, \frac{d(x)}{10 C_{1}}\right) \cap \Upsilon\right) \\
(\stackrel{8.13)}{\geq} & \tilde{C}^{-1}\left(\frac{10}{d(x)}\right)^{n(n+1)}\left(\frac{d(x)}{10}\right)^{n(n+1)}\left(\frac{1}{C_{2}}\right)^{n+1} \\
\geq & \tilde{C}^{-1} C_{2}^{-(n+1)} .
\end{aligned}
$$

We have proven the inequality (8.16) for all $x \in G$, so finally we deduce with (C) from page 64

$$
\begin{aligned}
\mu(G) & =\tilde{C} C_{2}^{(n+1)} \int_{G} \tilde{C}^{-1} C_{2}^{-(n+1)} \mathrm{d} \mu(x) \\
& \leq \tilde{C} C_{2}^{(n+1)} \int_{G} \int \ldots \int \mathcal{K}^{p}\left(y_{0}, \ldots, y_{n}, x\right) \mathrm{d} \mu\left(y_{0}\right) \ldots \mathrm{d} \mu\left(y_{n}\right) \mathrm{d} \mu(x) \\
& \leq C\left(N, n, \mathcal{K}, p, C_{0}\right) \mathcal{M}_{\mathcal{K}^{p}}(\mu) \\
& \stackrel{(\mathrm{C})}{\leq} C\left(N, n, \mathcal{K}, p, C_{0}\right) \eta .
\end{aligned}
$$

Lemma 8.2. Let $\alpha, \varepsilon>0$. If $\eta \leq 2 \varepsilon$, we have for all $x \in F \backslash G$ that

$$
(20 K)^{-1} d(x) \leq D(\pi(x)) \leq d(x),
$$

where $K$ is the constant defined on page 125.

Proof. Let $x \in F \backslash G$.

We have $D(\pi(x))=\inf _{y \in \pi^{-1}(\pi(x))} d(y) \leq d(x)$.

1. Case: $x \in \mathcal{Z}$.

With Lemma 6.11 , we get $d(x)=0$, so the statement is trivial.

2. Case: $x \notin \mathcal{Z}$.

Since $x \notin G \cup \mathcal{Z}$, by definition of $G$, there exists some $i \in I_{12}$ with $\pi(x) \in 3 R_{i}$ and $x \in K B_{i}$. We have $B_{i}=B\left(X_{i}, t_{i}\right)$ where $\left(X_{i}, t_{i}\right) \in S$ (see Lemma 6.15) and $K>1$ (see (8.1) on page 125) so we obtain

$$
d(x)=\inf _{(X, t) \in S}(d(X, x)+t) \stackrel{\left(X_{i}, t_{i}\right) \in S}{\leq} d\left(X_{i}, x\right)+t_{i} \leq K \frac{\operatorname{diam} B_{i}}{2}+\frac{\operatorname{diam} B_{i}}{2}<K \operatorname{diam} B_{i} .
$$


Now, with Lemma 6.14 (i) and 6.15, we deduce

$$
D(\pi(x)) \geq 10 \operatorname{diam} R_{i} \geq \frac{10}{200} \operatorname{diam} B_{i} \geq \frac{1}{20 K} d(x) .
$$

Lemma 8.3. Let $0<\alpha \leq \frac{1}{4}$. There exists some $\bar{\varepsilon}=\bar{\varepsilon}\left(N, n, C_{0}\right)$ and some $\tilde{k} \geq 4$ so that, if $\eta<2 \bar{\varepsilon}$ and $k \geq \tilde{k}$, for all $\varepsilon \in\left[\frac{\eta}{2}, \bar{\varepsilon}\right)$ we have that the following is true. There exists some constant $C=C(n)$ so that, for all $x \in F$ with $t \geq \frac{d(x)}{10}$, we have

$$
\int_{B(x, t) \backslash G} d(u, \pi(u)+A(\pi(u))) \mathrm{d} \mu(u) \leq C \varepsilon t^{n+1} .
$$

Proof. Let $0<\alpha \leq \frac{1}{4}$. We choose some $\varepsilon$ with $\eta \leq 2 \varepsilon<2 \bar{\varepsilon}$ and some $k \geq \tilde{k}:=\max \{\bar{k}, \tilde{C}\}$, where $\bar{\varepsilon}$ and $\bar{k}$ are given by Lemma 6.22 and $\tilde{C}$ is a fixed constant introduced in step VI of this proof.

Let $x \in F$ and $t \geq \frac{d(x)}{10}$. We define

$$
I(x, t):=\left\{i \in I_{12} \mid\left(3 R_{i} \times P_{0}^{\perp}\right) \cap B(x, t) \cap(F \backslash G) \neq \emptyset\right\}
$$

where $3 R_{i} \times P_{0}^{\perp}:=\left\{x \in \mathbb{R}^{N} \mid \pi(x) \in 3 R_{i}\right\}$. At first, we prove some intermediate results:

I. If $u \in \mathcal{Z}$, the definition of $A$ (see page 78) yields $d(u, \pi(u)+A(\pi(u)))=0$.

II. Let $u \in(B(x, t) \cap F) \backslash(G \cup \mathcal{Z})$. We have (cf. Lemma 6.20 and Definition 6.21)

$$
\begin{aligned}
d(u, \pi(u)+A(\pi(u))) & =d\left(\pi(u)+\pi^{\perp}(u), \pi(u)+A(\pi(u))\right) \\
& =\left|\pi^{\perp}(u)-A(\pi(u))\right| \\
& =\left|\sum_{i \in I_{12}} \phi_{i}(\pi(u)) \pi^{\perp}(u)-\sum_{j \in I_{12}} \phi_{j}(\pi(u)) A_{j}(\pi(u))\right| \\
& \leq \sum_{j \in I_{12}} \phi_{j}(\pi(u))\left|\pi^{\perp}(u)-A_{j}(\pi(u))\right| .
\end{aligned}
$$

III. We have $(B(x, t) \cap F) \backslash(G \cup \mathcal{Z}) \subset \bigcup_{i \in I(x, t)}\left(3 R_{i} \times P_{0}^{\perp}\right) \cap K B_{i}$.

Proof. Let $y \in(B(x, t) \cap F) \backslash(G \cup \mathcal{Z})$. Since $y \notin G \cup \mathcal{Z}$, by definition of $G$, there exists some $i \in I_{12}$ with $\pi(y) \in 3 R_{i}$ and $y \in K B_{i}$. Hence we obtain $i \in I(x, t)$ and

$$
y \in\left(3 R_{i} \times P_{0}^{\perp}\right) \cap K B_{i} .
$$

IV. Let $u \in 3 R_{i} \times P_{0}^{\perp}$. We have $\pi(u) \in 3 R_{i}$ and with Lemma 6.14 there exists at most $(180)^{n}$ cells $R_{j}$ with $3 R_{i} \cap 3 R_{j} \neq \emptyset$. So $\sum_{j \in I_{12}} \phi_{j}(\pi(u))$ is a finite sum $\left(\phi_{j}\right.$ is defined on page 78). 
V. Let $i \in I(x, t)$ and $j \in I_{12}$. If $u \in\left(3 R_{i} \times P_{0}^{\perp}\right) \cap K B_{i}$, then we have $\phi_{j}(\pi(u)) \leq \phi_{i, j}$, where

$$
\phi_{i, j}:= \begin{cases}1, & \text { if } 3 R_{i} \cap 3 R_{j} \neq \emptyset \\ 0, & \text { if } 3 R_{i} \cap 3 R_{j}=\emptyset .\end{cases}
$$

Proof. Let $\phi_{j}(\pi(u)) \neq 0$. The definition of $\phi_{j}$ (see page 78) gives us $\pi(u) \in 3 R_{j}$ and, because $\pi(u) \in 3 R_{i}$, we deduce $3 R_{i} \cap 3 R_{j} \neq \emptyset$. Hence we obtain $\phi_{j}(\pi(u)) \leq 1=\phi_{i, j}$.

VI. If $\phi_{i, j} \neq 0$, then we have $K B_{i} \subset k B_{j}$.

Proof. If $\phi_{i, j} \neq 0$, we can apply Lemma 6.14 (iii) and Lemma 6.22 (i). Hence, using Lemma 6.15 (i), the size of $B_{i}$ as well as the distance of $B_{i}$ to $B_{j}$ are comparable to the size of $B_{j}$. Consequently, there exists some constant $\tilde{C}$ so that $K B_{i} \subset \tilde{C} B_{j} \subset k B_{j}$.

VII. If $u \in k B_{j}$, we have $\left|\pi^{\perp}(u)-A_{j}(\pi(u))\right|<2 d\left(u, P_{j}\right)$. We recall that $P_{j}$ is the graph of the affine map $A_{j}$ (cf. Definition 6.18 and Lemma 6.19).

Proof. We set $\hat{P}_{0}:=P_{0}+A_{j}(\pi(u))$ and $v:=\pi(u)+A_{j}(\pi(u))=\pi_{\hat{P}_{0}}(u)$. Using Lemma 2.1 , we get

$$
\begin{aligned}
\left|\pi_{P_{j}}(u)-v\right| & =\left|\pi_{P_{j}}(u)-\pi_{\hat{P}_{0}}(u)\right| \\
& =\left|\pi_{P_{j}-v}(u-v)-\pi_{\hat{P}_{0}-v}(u-v)\right| \\
& =|u-v|\left|\pi_{P_{j}-v}\left(\frac{u-v}{|u-v|}\right)-\pi_{\hat{P}_{0}-v}\left(\frac{u-v}{|u-v|}\right)\right| \\
& \leq|u-v| \Varangle\left(P_{j}, P_{0}\right) .
\end{aligned}
$$

All in all, we obtain with $\Varangle\left(P_{j}, P_{0}\right) \leq \alpha<\frac{1}{2}$ (cf. Lemma 6.3 (vi) and Definition 6.18)

$$
|u-v| \leq\left|u-\pi_{P_{j}}(u)\right|+\left|\pi_{P_{j}}(u)-v\right|<d\left(u, P_{j}\right)+\frac{1}{2}|u-v|
$$

and hence

$$
\left|\pi^{\perp}(u)-A_{j}(\pi(u))\right|=|u-v|<2 d\left(u, P_{j}\right)
$$

VIII. Let $B_{j}=B\left(X_{j}, t_{j}\right)$. Since $\left(X_{j}, t_{j}\right) \in S \subset S_{\text {total }}$, by definition of $S_{\text {total }}$, we obtain $\beta_{1 ; k}^{P_{j}}\left(X_{j}, t_{j}\right) \leq 2 \varepsilon$.

IX. Let $i \in I(x, t)$ and $j \in I_{12}$. If $\phi_{i, j} \neq 0$, we conclude that $3 R_{i} \cap 3 R_{j} \neq \emptyset$. Hence, with Lemma 6.14 (iii) and Lemma 6.15 (i), we deduce $2 t_{j}=\operatorname{diam} B_{j} \leq 200 \operatorname{diam} R_{j} \leq$ $1000 \operatorname{diam} R_{i}$.

X. For $i \in I(x, t)$, we have with Lemma 6.14 (iv) that $\sum_{j \in I_{12}} \phi_{i, j} \leq(180)^{n}$. 
XI. For $i \in I(x, t)$, there exists some $y \in B(x, t) \cap(F \backslash G)$ with $\pi(y) \in 3 R_{i}$. We obtain with Lemma 6.14, Lemma 8.2 and our assumption $t \geq \frac{d(x)}{10}$ that

$$
10 \operatorname{diam} R_{i} \leq D(\pi(y)) \leq d(y) \leq d(x)+d(x, y) \leq 11 t .
$$

XII. We have $\sum_{i \in I(x, t)}\left(\operatorname{diam} R_{i}\right)^{n}<\sqrt{n}^{n} \omega_{n}(7 t)^{n}$.

Proof. Let $i \in I(x, t)$. With XI we obtain diam $R_{i}<2 t$ and, because $\left(3 R_{i} \times P_{0}^{\perp}\right) \cap$ $B(x, t) \neq \emptyset$, we get $R_{i} \subset B\left(\pi(x), t+\operatorname{diam} 3 R_{i}\right) \cap P_{0} \subset B(\pi(x), 7 t) \cap P_{0}$. Moreover, with Lemma 6.14 (ii), the primitive cells $R_{i}$ have disjoint interior and hence we get with Lemma A.7 (we recall that $\omega_{n}$ denotes the volume of the $n$-dimensional unit sphere)

$$
\begin{aligned}
\sum_{i \in I(x, t)}\left(\operatorname{diam} R_{i}\right)^{n} & =\sum_{i \in I(x, t)} \sqrt{n}^{n} \mathcal{H}^{n}\left(R_{i}\right) \\
& =\sqrt{n}^{n} \mathcal{H}^{n}\left(\bigcup_{i \in I(x, t)} R_{i}\right) \\
& \leq \sqrt{n}^{n} \mathcal{H}^{n}\left(B(\pi(x), 7 t) \cap P_{0}\right) \\
& =\sqrt{n}^{n} \omega_{n}(7 t)^{n} .
\end{aligned}
$$

Using all those results, we get

$$
\begin{aligned}
& \int_{B(x, t) \backslash G} d(u, \pi(u)+A(\pi(u))) \mathrm{d} \mu(u) \\
\stackrel{\mathrm{I}}{=} & \int_{B(x, t) \backslash(G \cup \mathcal{Z})} d(u, \pi(u)+A(\pi(u))) \mathrm{d} \mu(u) \\
\stackrel{\mathrm{II}}{\leq} & \int_{B(x, t) \backslash(G \cup \mathcal{Z})} \sum_{j \in I_{12}} \phi_{j}(\pi(u))\left|\pi^{\perp}(u)-A_{j}(\pi(u))\right| \mathrm{d} \mu(u) \\
\stackrel{\mathrm{III}}{\leq} & \sum_{i \in I(x, t)} \int_{\left(3 R_{i} \times P_{0}^{\perp}\right) \cap K B_{i}} \sum_{j \in I_{12}} \phi_{j}(\pi(u))\left|\pi^{\perp}(u)-A_{j}(\pi(u))\right| \mathrm{d} \mu(u) \\
\stackrel{\mathrm{IV}}{=} & \sum_{i \in I(x, t)} \sum_{j \in I_{12}} \int_{\left(3 R_{i} \times P_{0}^{\perp}\right) \cap K B_{i}} \phi_{j}(\pi(u))\left|\pi^{\perp}(u)-A_{j}(\pi(u))\right| \mathrm{d} \mu(u) \\
\stackrel{\mathrm{V}}{\leq} & \sum_{i \in I(x, t)} \sum_{j \in I_{12}} \phi_{i, j} \int_{K B_{i}}\left|\pi^{\perp}(u)-A_{j}(\pi(u))\right| \mathrm{d} \mu(u) \\
\mathrm{VI}, \mathrm{VII} & \sum_{i \in I(x, t)} \sum_{j \in I_{12}} \phi_{i, j} \int_{k B_{j}} 2 d\left(u, P_{j}\right) \mathrm{d} \mu(u) \\
= & 2 \sum_{i \in I(x, t)} \sum_{j \in I_{12}} \phi_{i, j} t_{j}^{n+1} \frac{1}{t_{j}^{n}} \int_{k B_{j}} \frac{d\left(u, P_{j}\right)}{t_{j}} \mathrm{~d} \mu(u)
\end{aligned}
$$




$$
\begin{aligned}
& =2 \sum_{i \in I(x, t)} \sum_{j \in I_{12}} \phi_{i, j} t_{j}^{n+1} \beta_{1 ; k}^{P_{j}}\left(X_{j}, t_{j}\right) \\
& \stackrel{\text { VIII }}{\leq} 2 \sum_{i \in I(x, t)} \sum_{j \in I_{12}} \phi_{i, j} t_{j}^{n+1} 2 \varepsilon \\
& \stackrel{\text { IX }}{\leq} C(n) \varepsilon \sum_{i \in I(x, t)}\left(\operatorname{diam} R_{i}\right)^{n+1} \sum_{j \in I_{12}} \phi_{i, j} \\
& \underset{\text { X }}{\leq} C(n) \varepsilon \sum_{i \in I(x, t)}\left(\operatorname{diam} R_{i}\right)^{n+1}(180)^{n} \\
& \underset{\leq}{\leq} C(n) \varepsilon t \sum_{i \in I(x, t)}\left(\operatorname{diam} R_{i}\right)^{n} \\
& \underset{\mathrm{XII}}{\leq} C(n) \varepsilon t^{n+1} .
\end{aligned}
$$

Definition 8.4. We define

$$
\tilde{F}:=\left\{x \in F \backslash G \mid d(x, \pi(x)+A(\pi(x))) \leq \varepsilon^{\frac{1}{2}} d(x)\right\} .
$$

Theorem 8.5. Let $0<\alpha \leq \frac{1}{4}$. There exists some $\hat{\varepsilon}=\hat{\varepsilon}\left(N, n, C_{0}\right) \leq \frac{1}{4}$ and some $\tilde{k} \geq 4$ so that, if $\eta<2 \hat{\varepsilon}$ and $k \geq \tilde{k}$, there exists some constant $C_{5}=C_{5}\left(N, n, \mathcal{K}, p, C_{0}\right)$ so that, for all $\varepsilon \in\left[\frac{\eta}{2}, \hat{\varepsilon}\right)$, we have $\mu(F \backslash \tilde{F}) \leq C_{5} \varepsilon^{\frac{1}{2}}$.

Proof. Let $0<\alpha \leq \frac{1}{4}$. We choose some $\varepsilon$ with $\eta \leq 2 \varepsilon<2 \hat{\varepsilon}:=\min \left\{2 \tilde{\varepsilon}, 2 \bar{\varepsilon}, \frac{1}{2}\right\}$ and some $k \geq \tilde{k}$ where $\tilde{\varepsilon}$ is given by Lemma 8.1 and $\bar{\varepsilon}$ and $\tilde{k}$ are given by Lemma 8.3.

At first, we prove some intermediate results:

I. We have $\mathcal{Z} \subset \tilde{F}$ because for $x \in \mathcal{Z}$ the definition of $A$ on $\mathcal{Z}$ (see Definition 78) implies that $d(x, \pi(x)+A(\pi(x)))=d(x, x)=0$.

II. If $x \in F \backslash(\tilde{F} \cup G)$, we conclude with I that $x \notin \mathcal{Z}$ and, with Lemma 6.11 , we deduce $d(x) \neq 0$. So

$$
\mathcal{G}=\left\{B\left(x, \frac{d(x)}{10}\right) \mid x \in F \backslash(\tilde{F} \cup G)\right\}
$$

is a set of nondegenerate balls. For $x \in F \subset B(0,5)$, we have $d(x) \leq 60$ (see Lemma 6.10) so that we can apply the Besicovitch's covering theorem A.12 to $\mathcal{G}$ and get $N_{0}=N_{0}(N)$ families

$$
\mathcal{B}_{m} \subset \mathcal{G} \quad m=1, \ldots, N_{0}
$$

of disjoint balls with

$$
F \backslash(\tilde{F} \cup G) \subset \bigcup_{m=1}^{N_{0}} \bigcup_{B \in \mathcal{B}_{m}} B
$$


III. Since $d$ is 1-Lipschitz (Lemma 6.9), we obtain for all $u \in B\left(x, \frac{d(x)}{10}\right)$

$$
d(x)-d(u) \leq|d(x)-d(u)| \leq d(x, u) \leq \frac{d(x)}{10}
$$

and hence

$$
\frac{1}{d(u)} \leq \frac{10}{9} \frac{1}{d(x)}<\frac{2}{d(x)}
$$

IV. Let $1 \leq m \leq N_{0}$ and let $B_{x}=B\left(x, \frac{d(x)}{10}\right)$ and $B_{y}=B\left(y, \frac{d(y)}{10}\right)$ be two balls in $\mathcal{B}_{m}$. Then we either have

a) $\pi\left(\frac{1}{40 K} B_{x}\right) \cap \pi\left(\frac{1}{40 K} B_{y}\right)=\emptyset$

or

b) if $2 d(x) \geq d(y)$, we have $B_{y} \subset 200 B_{x}$ and $\operatorname{diam} B_{y}>(40 K)^{-1} \operatorname{diam} B_{x}$, where $K$ is the constant from page 125 .

Proof. Let $\pi\left(\frac{1}{40 K} B_{x}\right) \cap \pi\left(\frac{1}{40 K} B_{y}\right) \neq \emptyset$ and $2 d(x) \geq d(y)$. We deduce with Lemma 6.12

$$
d(x, y) \leq 6(d(x)+d(y))+2 d(\pi(x), \pi(y))<(18+1) d(x)
$$

which implies

$$
B_{y} \subset B\left(x, 19 d(x)+\frac{d(y)}{10}\right) \subset B(x, 20 d(x))=200 B_{x} .
$$

With Lemma 8.2, we get

$$
\frac{d(x)}{20 K} \leq D(\pi(x)) \leq D(\pi(y))+d(\pi(x), \pi(y)) \leq d(y)+\frac{3 d(x)}{40 K \cdot 10}<d(y)+\frac{d(x)}{40 K},
$$

and hence

$$
d(y)>\left(\frac{1}{20 K}-\frac{1}{40 K}\right) d(x)=(40 K)^{-1} d(x) .
$$

All in all, we have proven that either case a) or case b) is true.

V. There exists some constant $C=C(n)$ so that, for all $1 \leq m \leq N_{0}$, we have

$$
\sum_{B \in \mathcal{B}_{m}}(\operatorname{diam} B)^{n} \leq C
$$

Proof. Let $1 \leq m \leq N_{0}$. We recursively construct for every $m$ some sequence of numbers, some sequence of balls and some sequence of sets. At first, we define the initial elements. Let $d_{m}^{1}:=\sup _{B \in \mathcal{B}_{m}} \operatorname{diam} B$. We have $d_{m}^{1}<\infty$ because, for all $x \in F \subset B(0,5)$, we have with Lemma 6.10 that $d(x) \leq 60$. Now we choose $B_{m}^{1} \in \mathcal{B}_{m}$ with $\operatorname{diam} B_{m}^{1} \geq \frac{d_{m}^{1}}{2}$ and define

$$
\mathcal{B}_{m}^{1}:=\left\{B \in \mathcal{B}_{m} \mid \pi\left(\frac{1}{40 K} B_{m}^{1}\right) \cap \pi\left(\frac{1}{40 K} B\right) \neq \emptyset\right\} .
$$


We continue this sequences recursively. We set

$$
d_{m}^{i+1}=\sup _{B^{\prime} \in \mathcal{B}_{m} \backslash \bigcup_{j=1}^{i} \mathcal{B}_{m}^{j}} \operatorname{diam} B^{\prime}
$$

choose

$$
B_{m}^{i+1} \in \mathcal{B}_{m} \backslash \bigcup_{j=1}^{i} \mathcal{B}_{m}^{j}
$$

with $\operatorname{diam} B_{m}^{i+1} \geq \frac{d_{m}^{i+1}}{2}$ and define

$$
\mathcal{B}_{m}^{i+1}:=\left\{B \in \mathcal{B}_{m} \backslash \bigcup_{j=1}^{i} \mathcal{B}_{m}^{j} \mid \pi\left(\frac{1}{40 K} B_{m}^{i+1}\right) \cap \pi\left(\frac{1}{40 K} B\right) \neq \emptyset\right\} .
$$

If there exists some $l \in \mathbb{N}$ so that eventually $\mathcal{B}_{m} \backslash \bigcup_{j=1}^{l} \mathcal{B}_{m}^{j}=\emptyset$, we set for all $i \geq l$ $\mathcal{B}_{m}^{i}:=\emptyset$, and interrupt the sequences $\left(d_{m}^{i}\right)$ and $\left(B_{m}^{i}\right)$. We have the following results:

(i) For all $l \in \mathbb{N}$ and $B_{m}^{l}=B\left(x_{m}^{l}, \frac{d\left(x_{m}^{l}\right)}{10}\right)$, we have with Lemma 6.10 and $x_{m}^{l} \in F \subset$ $B(0,5)$ that $\frac{d\left(x_{m}^{l}\right)}{10} \leq 6$. Hence we get $B_{m}^{l} \subset B(0,11)$.

(ii) For all $1 \leq m \leq N_{0}$, we have

$$
\bigcup_{i=1}^{\infty} \mathcal{B}_{m}^{i}=\mathcal{B}_{m}
$$

Proof. 1. Case: There exist only finitely many $d_{m}^{l}$.

This means that there exists some $l \in \mathbb{N}$ with $\mathcal{B}_{m} \backslash \bigcup_{j=1}^{l} \mathcal{B}_{m}^{j}=\emptyset$, which implies $\mathcal{B}_{m} \subset \bigcup_{j=1}^{\infty} \mathcal{B}_{m}^{j}$.

2. Case: There exist infinitely many $d_{m}^{l}$.

Since $\mathcal{B}_{m}$ is a family of disjoint balls, the set $\left\{B_{m}^{l} \mid l \in \mathbb{N}\right\}$ is also a family of disjoint balls. Due to (i), all of those balls are contained in $B(0,11)$. If there exists some $c>0$ with $\operatorname{diam} B_{m}^{l}>c$ for all $l \in \mathbb{N}$, there can not be infinitely many of such balls. Hence we deduce

$$
\operatorname{diam} B_{m}^{l} \rightarrow 0 \quad \text { if } l \rightarrow \infty .
$$

Let $B \in \mathcal{B}_{m}$. We assume that

$$
B \notin \bigcup_{i=1}^{\infty} \mathcal{B}_{m}^{i}
$$

We obtain for all $l \in \mathbb{N}$

$$
\operatorname{diam} B_{m}^{l} \geq \frac{d_{m}^{l}}{2}=\frac{1}{2} \sup _{B^{\prime} \in \mathcal{B}_{m} \backslash \bigcup_{j=1}^{l-1} \mathcal{B}_{m}^{j}} \operatorname{diam} B^{\prime} \geq \frac{\operatorname{diam} B}{2},
$$

which is in contradiction to (8.17). So we get $B \in \bigcup_{i=1}^{\infty} \mathcal{B}_{m}^{i}$.

All in all, we have proven $\bigcup_{i=1}^{\infty} \mathcal{B}_{m}^{i} \supset \mathcal{B}_{m}$.

The inverse inclusion follows by definition of $\mathcal{B}_{m}^{i}$. 
(iii) We have $\# \mathcal{B}_{m}^{l} \leq(200 \cdot 80 K)^{n}$ for all $1 \leq m \leq N_{0}$ and for all $l \in \mathbb{N}$.

Proof. Let $1 \leq m \leq N_{0}, l \in \mathbb{N}$ and $B_{y}=B\left(y, \frac{d(y)}{10}\right) \in \mathcal{B}_{m}^{l}, B_{m}^{l}=B\left(x_{m}^{l}, \frac{d\left(x_{m}^{l}\right)}{10}\right) \in$ $\mathcal{B}_{m}^{l}$. We have $\pi\left(\frac{1}{40 K} B_{m}^{l}\right) \cap \pi\left(\frac{1}{40 K} B_{y}\right) \neq \emptyset$ and

$$
2 d\left(x_{m}^{l}\right)=10 \operatorname{diam} B_{m}^{l} \geq 10 \frac{d_{m}^{l}}{2} \geq 10 \frac{\operatorname{diam} B_{y}}{2}=d(y) .
$$

Hence we obtain with IV

$$
\begin{aligned}
B_{y} & \subset 200 B_{m}^{l}, \\
\operatorname{diam} B_{y} & >(40 K)^{-1} \operatorname{diam} B_{m}^{l} .
\end{aligned}
$$

The balls in $\mathcal{B}_{m}^{l}$ are disjoint, so, with Lemma A.4 $\left(s=\frac{\operatorname{diam} B_{m}^{l}}{80 K}, r=200 \frac{\text { diam } B_{m}^{l}}{2}\right)$, we deduce

$$
\# \mathcal{B}_{m}^{l} \leq\left(\frac{r}{s}\right)^{N} \leq(200 \cdot 80 K)^{N}
$$

(iv) We have

$$
\sum_{l=1}^{\infty}\left(\operatorname{diam} \pi\left(\frac{1}{40 K} B_{m}^{l}\right)\right)^{n} \leq 22^{n} .
$$

Proof. Let $l_{1} \neq l_{2}$. We have

$$
\pi\left(\frac{1}{40 K} B_{m}^{l_{1}}\right) \cap \pi\left(\frac{1}{40 K} B_{m}^{l_{2}}\right)=\emptyset .
$$

Furthermore, we get with (i) for all $l \in \mathbb{N}$

$$
\pi\left(\frac{1}{40 K} B_{m}^{l}\right) \subset \pi(B(0,11)),
$$

so that

$$
\begin{aligned}
\sum_{l=1}^{\infty}\left(\operatorname{diam} \pi\left(\frac{1}{40 K} B_{m}^{l}\right)\right)^{n} & =\frac{2^{n}}{\omega_{n}} \sum_{l=1}^{\infty} \mathcal{H}^{n}\left(\pi\left(\frac{1}{40 K} B_{m}^{l}\right)\right) \\
& =\frac{2^{n}}{\omega_{n}} \mathcal{H}^{n}\left(\bigcup_{l=1}^{\infty} \pi\left(\frac{1}{40 K} B_{m}^{l}\right)\right) \\
& \leq \frac{2^{n}}{\omega_{n}} \mathcal{H}^{n}(\pi(B(0,11))) \\
& =22^{n} .
\end{aligned}
$$


Now we are able to prove V:

$$
\begin{aligned}
\sum_{B \in \mathcal{B}_{m}}(\operatorname{diam} B)^{n} & \stackrel{(\mathrm{ii})}{=} \sum_{l=1}^{\infty} \sum_{B \in \mathcal{B}_{m}^{l}}(\operatorname{diam} B)^{n} \\
& \leq \sum_{l=1}^{\infty} \sum_{B \in \mathcal{B}_{m}^{l}}\left(d_{m}^{l}\right)^{n} \\
& \stackrel{(\mathrm{iii})}{\leq}(200 \cdot 80 K)^{n} 2^{n} \sum_{l=1}^{\infty}\left(\operatorname{diam} B_{m}^{l}\right)^{n} \\
& =(200 \cdot 80 K)^{n} 2^{n}(40 K)^{n} \sum_{l=1}^{\infty}\left(\operatorname{diam} \pi\left(\frac{1}{40 K} B_{m}^{l}\right)\right)^{n} \\
& \stackrel{\text { (iv) }}{\leq} C(n) .
\end{aligned}
$$

Finally, we can finish the proof of Theorem 8.5. Let $p_{B}$ denote the centre of some ball $B$. Using the definition of $\tilde{F}$ and Lemma 8.3, there exists some constant $C=C(n)$ so that we obtain

$$
\begin{aligned}
\varepsilon^{\frac{1}{2}} \mu(F \backslash(\tilde{F} \cup G)) & =\int_{F \backslash(\tilde{F} \cup G)} \varepsilon^{\frac{1}{2}} \mathrm{~d} \mu(u) \\
& <\int_{F \backslash(\tilde{F} \cup G)} \frac{d(u, \pi(u)+A(\pi(u)))}{d(u)} \mathrm{d} \mu(u) \\
& \leq \sum_{m=1}^{\text {II }} \sum_{B \in \mathcal{B}_{m}} \int_{B \backslash(\tilde{F} \cup G)} \frac{d(u, \pi(u)+A(\pi(u)))}{d(u)} \mathrm{d} \mu(u) \\
& <\sum_{m=1}^{N_{0}} \sum_{B \in \mathcal{B}_{m}} \frac{2}{d\left(p_{B}\right)} \int_{B \backslash G} d(u, \pi(u)+A(\pi(u))) \mathrm{d} \mu(u) \\
& \leq \sum_{m=1}^{N_{0}} \sum_{B \in \mathcal{B}_{m}} \frac{2}{d\left(p_{B}\right)} C \varepsilon\left(\frac{\operatorname{diam} B}{2}\right)^{n+1} \\
& =C \varepsilon \sum_{m=1}^{N_{0}} \sum_{B \in \mathcal{B}_{m}} \frac{2}{d\left(p_{B}\right)} \frac{d\left(p_{B}\right)}{10}\left(\frac{\operatorname{diam} B}{2}\right)^{n} \\
& \stackrel{\mathrm{V}}{\leq} C(N, n) \varepsilon .
\end{aligned}
$$

This leads to $\mu(F \backslash(\tilde{F} \cup G)) \leq C(N, n) \varepsilon^{\frac{1}{2}}$. With $\eta<2 \varepsilon \leq \varepsilon^{\frac{1}{2}}$ and Lemma 8.1, we get $\mu(G) \leq C\left(N, n, \mathcal{K}, p, C_{0}\right) \varepsilon^{\frac{1}{2}}$. All in all, we deduce

$$
\mu(F \backslash \tilde{F}) \leq \mu(F \backslash(\tilde{F} \cup G))+\mu(G) \leq C\left(N, n, \mathcal{K}, p, C_{0}\right) \varepsilon^{\frac{1}{2}} .
$$




\section{2 $F_{1}$ is small}

Now we are able to estimate $\mu\left(F_{1}\right)$. We recall that $\eta$ and $k$ are fixed constants (cf. the first lines of section 6.1), and (cf. Definition 6.4)

$$
F_{1}=\left\{x \in F \backslash \mathcal{Z} \mid \exists y \in F, \exists \tau \in\left[\frac{h(x)}{5}, \frac{h(x)}{2}\right], d(x, y) \leq \frac{\tau}{2} \text { and } \delta(B(y, \tau)) \leq \delta\right\}
$$

where this definition depends on the choice of $\alpha, \varepsilon>0$.

Theorem 8.6. Let $0<\alpha \leq \frac{1}{4}$. There exist some $\varepsilon^{*}=\varepsilon^{*}\left(N, n, C_{0}\right)$ and some $\tilde{k} \geq 4$ so that, if $\eta<2 \varepsilon^{*}$ and $k \geq \tilde{k}$, for all $\varepsilon \in\left[\frac{\eta}{2}, \varepsilon^{*}\right)$, we have $\mu\left(F_{1}\right)<10^{-6}$.

Proof. Let $0<\alpha \leq \frac{1}{4}$ and let $\hat{\varepsilon}, C_{5}$ and $\tilde{k}$ be the constants given by Theorem 8.5. We set $\varepsilon^{*}:=\min \left\{\hat{\varepsilon}, \frac{10^{-14}}{C_{5}^{2}}\right\}$ and choose some $k \geq \tilde{k}$ and some $\varepsilon \in\left[\frac{\eta}{2}, \varepsilon^{*}\right)$. At first, we prove some intermediate results:

I. Let

$$
\mathcal{G}=\left\{B\left(x, \frac{h(x)}{10}\right) \mid x \in F_{1} \cap \tilde{F}\right\}
$$

This is a set of nondegenerate balls because $\mathcal{Z} \cap F_{1}=\emptyset$ and, by definition of $h(\cdot)$ (see page 65 ), we get $h(x) \leq 50$ for all $x \in F$. With Besicovitch's covering theorem A.12, there exist $N_{0}=N_{0}(N)$ families $\mathcal{B}_{m} \subset \mathcal{G}, m=1, \ldots, N_{0}$, containing countably many disjoint balls with

$$
F_{1} \cap \tilde{F} \subset \bigcup_{m=1}^{N_{0}} \bigcup_{B \in \mathcal{B}_{m}} B
$$

II. If $1 \leq m \leq N_{0}$ and $B \in \mathcal{B}_{m}$, we have $\mu(B) \leq 3^{n} \delta(\operatorname{diam} B)^{n}$.

Proof. Let $x \in F_{1} \cap \tilde{F}$ such that $B=B\left(x, \frac{h(x)}{10}\right)$. Due to the definition of $F_{1}$, there exists some $y \in F$ and some $\tau \in\left[\frac{h(x)}{5}, \frac{h(x)}{2}\right]$ with $d(x, y) \leq \frac{\tau}{2}$ and $\delta(B(y, \tau)) \leq \delta$. For any $z \in B$, we get

$$
d(z, y) \leq d(z, x)+d(x, y) \leq \frac{h(x)}{10}+\frac{\tau}{2} \leq \frac{\tau}{2}+\frac{\tau}{2}=\tau .
$$

Hence we obtain $B \subset B(y, \tau)$ and conclude

$$
\mu(B) \leq \mu(B(y, \tau)) \leq \delta \tau^{n} \leq \delta\left(\frac{h(x)}{2}\right)^{n}<3^{n} \delta(\operatorname{diam} B)^{n} .
$$

III. For all $1 \leq m \leq N_{0}$, we have $\sum_{B \in \mathcal{B}_{m}}(\operatorname{diam} B)^{n} \leq 192^{n}$. 


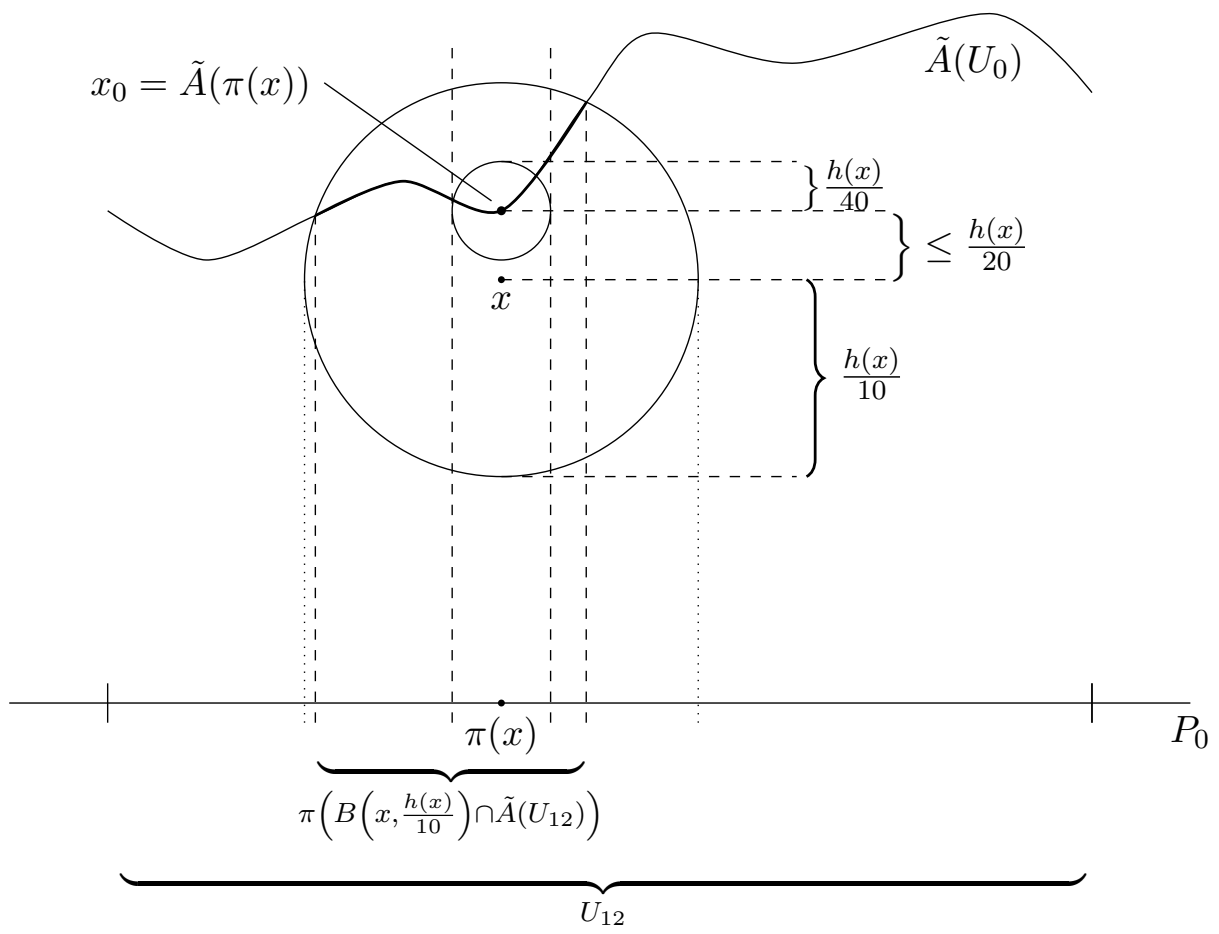

Figure 8.1: $\pi\left(B\left(x_{0}, \frac{h(x)}{40}\right)\right) \subset \pi\left(B\left(x, \frac{h(x)}{10}\right) \cap \tilde{A}\left(U_{12}\right)\right)$

Proof. We define the function

$$
\tilde{A}: U_{12} \rightarrow \mathbb{R}^{N}, u \mapsto u+A(u),
$$

where $U_{12}=B(0,12) \cap P_{0} . \tilde{A}$ is Lipschitz continuous with Lipschitz constant less than 2 because $A$ is defined on $U_{12}$ (see page 78), $3 \alpha$-Lipschitz continuous (see Lemma 6.28) and $\alpha \leq \frac{1}{4}$. Let $B=B\left(x, \frac{h(x)}{10}\right) \in \mathcal{B}_{m}$. We have $F \subset B(0,5)$ (see (A) on page 64) and so $\pi(F) \subset P_{0} \cap B(0,5)$ because $\pi$ is the orthogonal projection on $P_{0}$ and $0 \in P_{0}$. With the definition of $\tilde{F}$ and Lemma 6.11 , we obtain for $x_{0}:=\tilde{A}(\pi(x))$

$$
d\left(x, x_{0}\right)=d(x, \pi(x)+A(\pi(x))) \leq \varepsilon^{\frac{1}{2}} d(x) \leq \varepsilon^{\frac{1}{2}} h(x)<\frac{h(x)}{20} .
$$

For some $z \in \pi\left(B\left(x_{0}, \frac{h(x)}{40}\right)\right) \subset U_{12}$, we get using $\pi(x)=\pi\left(x_{0}\right)$

$$
d(\tilde{A}(z), x) \leq d\left(\tilde{A}(z), \tilde{A}\left(\pi\left(x_{0}\right)\right)\right)+d\left(x_{0}, x\right)<2 d\left(z, \pi\left(x_{0}\right)\right)+\frac{h(x)}{20} \leq \frac{h(x)}{10},
$$

which implies $\tilde{A}\left(\pi\left(B\left(x_{0}, \frac{h(x)}{40}\right)\right)\right) \subset B \cap \tilde{A}\left(U_{12}\right)$, and hence we gain $\pi\left(B\left(x_{0}, \frac{h(x)}{40}\right)\right) \subset$ $\pi\left(B \cap \tilde{A}\left(U_{12}\right)\right)$. Now we have with [EG92, 2.4.1, Thm. 1]

$$
\frac{\omega_{n}}{8^{n}}(\operatorname{diam} B)^{n}=\omega_{n}\left(\frac{h(x)}{40}\right)^{n}=\mathcal{H}^{n}\left(\pi\left(B\left(x_{0}, \frac{h(x)}{40}\right)\right)\right) \leq \mathcal{H}^{n}\left(B \cap \tilde{A}\left(U_{12}\right)\right) .
$$


Using [EG92, 2.4.1, Thm. 1] again, we obtain

$$
\mathcal{H}^{n}\left(\tilde{A}\left(U_{12}\right)\right) \leq\left(\operatorname{Lip}_{\tilde{A}}\right)^{n} \mathcal{H}^{n}\left(U_{12}\right) \leq 24^{n} \omega_{n} .
$$

The balls in $\mathcal{B}_{m}$ are disjoint, so we conclude

$$
\sum_{B \in \mathcal{B}_{m}}(\operatorname{diam} B)^{n} \stackrel{(8.18)}{\leq} \frac{8^{n}}{\omega_{n}} \sum_{B \in \mathcal{B}_{m}} \mathcal{H}^{n}\left(B \cap \tilde{A}\left(U_{12}\right)\right) \leq \frac{8^{n}}{\omega_{n}} \mathcal{H}^{n}\left(\tilde{A}\left(U_{12}\right)\right) \stackrel{(8.19)}{\leq} 192^{n}
$$

Now we have

$$
\mu\left(F_{1} \cap \tilde{F}\right) \stackrel{\text { I }}{\leq} \sum_{m=1}^{N_{0}} \sum_{B \in \mathcal{B}_{m}} \mu(B) \stackrel{\text { II }}{\leq} 3^{n} \delta \sum_{m=1}^{N_{0}} \sum_{B \in \mathcal{B}_{m}}(\operatorname{diam} B)^{n} \stackrel{\mathrm{III}}{\leq} \delta N_{0} \cdot 576^{n} .
$$

Since $\delta \leq \frac{10^{-10}}{600^{n} N_{0}}$ (see (6.2) on page 65) and $\varepsilon^{\frac{1}{2}}<\frac{10^{-7}}{C_{5}}$, we deduce together with Theorem 8.5 that

$$
\mu\left(F_{1}\right) \leq \mu\left(F_{1} \cap \tilde{F}\right)+\mu(F \backslash \tilde{F})<10^{-6} .
$$

\section{3 $F_{2}$ is small}

We recall that $0<\eta \leq 2^{-(n+1)}$ and $k \geq 1$ are fixed constants (cf. the first lines of section 6.1) and that (cf. Definition 6.4)

$$
F_{2}=\left\{\begin{array}{l|l}
x \in F \backslash\left(\mathcal{Z} \cup F_{1}\right) \mid \begin{array}{l}
\exists y \in F, \exists \tau \in\left[\frac{h(x)}{5}, \frac{h(x)}{2}\right], \text { with } d(x, y) \leq \frac{\tau}{2} \\
\text { and } \\
\beta_{1 ; k}(y, \tau) \geq \varepsilon
\end{array}
\end{array}\right\}
$$

where this definition depends on the choice of $\alpha, \varepsilon>0$.

Theorem 8.7. Let $\alpha, \varepsilon>0$. There exists some constant $C=C\left(N, n, \mathcal{K}, p, C_{0}, k\right)$ so that, if $\eta \leq \frac{\varepsilon^{p}}{C} 10^{-6}$, we have

$$
\mu\left(F_{2}\right) \leq 10^{-6}
$$

Proof. Let $x \in F_{2}$ and $t \in(h(x), 2 h(x))$. It follows that $x \notin F_{1} \cup \mathcal{Z}$ and hence, for all $y \in F$ and for all $\tau \in\left[\frac{h(x)}{5}, \frac{h(x)}{2}\right]$ with $d(x, y) \leq \frac{\tau}{2}$, we obtain $\delta(B(y, \tau))>\delta$. So, in particular, we get $\delta\left(B\left(x, \frac{h(x)}{2}\right)\right)>\delta$ for $x=y$ and $\tau=\frac{h(x)}{2}$ and hence with $k_{0}=1$

$$
\begin{aligned}
\tilde{\delta}_{k_{0}}(B(x, t)) & =\sup _{z \in B\left(x, k_{0} t\right)} \delta(B(z, t)) \geq \delta(B(x, t))=\frac{\mu(B(x, t))}{t^{n}} \\
& \geq \frac{\mu\left(B\left(x, \frac{h(x)}{2}\right)\right)}{\left(4 \frac{h(x)}{2}\right)^{n}}>\frac{\delta}{4^{n}} .
\end{aligned}
$$


Let $(y, \tau)$ as in the definition of $F_{2}$. Then we have $d(x, y)+\tau<2 \tau \leq h(x)<t$ and hence $B(y, \tau) \subset B(x, t)$. We conclude

$$
\beta_{1 ; k}(x, t) \geq\left(\frac{\tau}{t}\right)^{n+1} \beta_{1 ; k}(y, \tau) \geq\left(\frac{\frac{h(x)}{5}}{2 h(x)}\right)^{n+1} \varepsilon=\frac{\varepsilon}{10^{n+1}} .
$$

Now, with Corollary $5.8\left(\lambda=\frac{\delta}{4^{n}}, k_{0}=1\right)$, there exists some constant $C=C\left(N, n, \mathcal{K}, p, C_{0}, k\right)$ so that

$$
\begin{aligned}
\mathcal{M}_{\mathcal{K}^{p}}(\mu) & \geq \frac{1}{C} \int_{F} \int_{0}^{\infty} \beta_{1 ; k}(x, t)^{p} \mathbb{1}_{\left\{\tilde{\delta}_{k_{0}}(B(x, t)) \geq \frac{\delta}{4^{n}}\right\}} \frac{\mathrm{d} t}{t} \mathrm{~d} \mu(x) \\
& \geq \frac{1}{C} \int_{F_{2}} \int_{h(x)}^{2 h(x)} \beta_{1 ; k}(x, t)^{p} \mathbb{1}_{\left\{\tilde{\delta}_{k_{0}}(B(x, t)) \geq \frac{\delta}{4^{n}}\right\}} \frac{\mathrm{d} t}{t} \mathrm{~d} \mu(x) \\
& \stackrel{(8.20)}{=} \frac{1}{C} \int_{F_{2}} \int_{h(x)}^{2 h(x)} \beta_{1 ; k}(x, t)^{p} \frac{\mathrm{d} t}{t} \mathrm{~d} \mu(x) \\
& \stackrel{(8.21)}{\geq} \frac{1}{C} \int_{F_{2}} \int_{h(x)}^{2 h(x)}\left(\frac{\varepsilon}{10^{n+1}}\right)^{p} \frac{\mathrm{d} t}{t} \mathrm{~d} \mu(x) \\
& \geq \frac{1}{C}\left(\frac{\varepsilon}{10^{n+1}}\right)^{p} \mu\left(F_{2}\right) \ln (2) .
\end{aligned}
$$

Finally, with condition (C) from page 64 and using that $\eta \leq \frac{\ln (2)}{10^{p(n+1)} C} \varepsilon^{p} 10^{-6}$, we get

$$
\mu\left(F_{2}\right) \leq \frac{C}{\ln (2)}\left(\frac{10^{n+1}}{\varepsilon}\right)^{p} \mathcal{M}_{\mathcal{K}^{p}}(\mu) \leq \frac{10^{p(n+1)} C}{\ln (2)} \varepsilon^{-p} \eta \leq 10^{-6} .
$$

\section{$8.4 F_{3}$ is small}

We set

$$
\tilde{\tilde{F}}:=\left\{x \in \tilde{F} \mid \mu(\tilde{F} \cap B(x, t)) \geq \frac{99}{100} \mu(F \cap B(x, t)) \text { for all } t \in(0,2)\right\} .
$$

We have defined $\tilde{F}$ on page 134 in Definition 8.4. To review

$$
\tilde{F}=\left\{x \in F \backslash G \mid d(x, \pi(x)+A(\pi(x))) \leq \varepsilon^{\frac{1}{2}} d(x)\right\} .
$$

Lemma 8.8. Let $0<\alpha \leq \frac{1}{4}$. There exists some $\hat{\varepsilon}=\hat{\varepsilon}\left(N, n, C_{0}\right) \leq \frac{1}{4}$ and some $\tilde{k} \geq 4$ so that, if $\eta<2 \hat{\varepsilon}$ and $k \geq \tilde{k}$, there exists some constant $C=C\left(N, n, \mathcal{K}, p, C_{0}\right)$ so that, for all $\varepsilon \in\left[\frac{\eta}{2}, \hat{\varepsilon}\right)$, we have

$$
\mu(F \backslash \tilde{\tilde{F}}) \leq C \varepsilon^{\frac{1}{2}} .
$$

Proof. Let $0<\alpha \leq \frac{1}{4}$ and choose $\hat{\varepsilon}, \tilde{k}$ to be the constants given by Theorem 8.5 and let $k \geq \tilde{k}$, $\eta \leq 2 \varepsilon<2 \hat{\varepsilon}$. We have $\tilde{\tilde{F}} \subset \tilde{F} \subset F$ and hence

$$
\mu(F \backslash \tilde{\tilde{F}}) \leq \mu(F \backslash \tilde{F})+\mu(\tilde{F} \backslash \tilde{\tilde{F}}) .
$$


In Theorem 8.5, we have already proven that $\mu(F \backslash \tilde{F}) \leq C\left(N, n, \mathcal{K}, p, C_{0}\right) \varepsilon^{\frac{1}{2}}$, so we only have to consider $\mu(\tilde{F} \backslash \tilde{\tilde{F}})$. For all $x \in \tilde{F} \backslash \tilde{\tilde{F}}$, there exists some $t_{x} \in(0,2)$ with

$$
\begin{aligned}
\mu\left(\tilde{F} \cap B\left(x, t_{x}\right)\right) & <\frac{99}{100} \mu\left(F \cap B\left(x, t_{x}\right)\right) \\
& \leq \frac{99}{100}\left(\mu\left((F \backslash \tilde{F}) \cap B\left(x, t_{x}\right)\right)+\mu\left(\tilde{F} \cap B\left(x, t_{x}\right)\right)\right)
\end{aligned}
$$

and so

$$
\mu\left(\tilde{F} \cap B\left(x, t_{x}\right)\right) \leq 99 \mu\left((F \backslash \tilde{F}) \cap B\left(x, t_{x}\right)\right) .
$$

Moreover, we have

$$
\tilde{F} \backslash \tilde{\tilde{F}} \subset \bigcup_{x \in \tilde{F} \backslash \tilde{\tilde{F}}} \tilde{F} \cap B\left(x, t_{x}\right),
$$

so with Besicovitch's covering theorem A.12 there exist $N_{0}=N_{0}(N)$ families $\mathcal{B}_{m}, m=1, . ., N_{0}$, of disjoint balls $B\left(x, t_{x}\right)$ so that

$$
\tilde{F} \backslash \tilde{\tilde{F}} \subset \bigcup_{m=1}^{N_{0}} \bigcup_{B \in \mathcal{B}_{m}}^{\cdot} \tilde{F} \cap B
$$

Finally, with Theorem 8.5, there exists some constant $C=C\left(N, n, \mathcal{K}, p, C_{0}\right)$ so that

$$
\mu(\tilde{F} \backslash \tilde{\tilde{F}}) \leq \sum_{m=1}^{N_{0}} \sum_{B \in \mathcal{B}_{m}} \mu(\tilde{F} \cap B) \stackrel{(8.22)}{\leq} 99 \sum_{m=1}^{N_{0}} \sum_{B \in \mathcal{B}_{m}} \mu((F \backslash \tilde{F}) \cap B) \leq 99 N_{0} \mu(F \backslash \tilde{F}) \leq C \varepsilon^{\frac{1}{2}} .
$$

Lemma 8.9. Let $\theta, \alpha>0$. There exist some constant $C=C\left(N, n, C_{0}, \theta\right)>1$ and some constant $\varepsilon_{0}=\varepsilon_{0}\left(N, n, C_{0}, \theta\right)>0$ so that, if $\eta<2 \varepsilon_{0}$ and $k \geq 4$, we have for all $\varepsilon \in\left[\frac{\eta}{2}, \varepsilon_{0}\right)$ that the following is true. If $(x, t) \in S$ and $100 t \geq \theta$, then we have $\Varangle\left(P_{(x, t)}, P_{0}\right) \leq C \varepsilon$.

Proof. Let $\theta, \alpha>0, k \geq 4$ and $\eta<2 \varepsilon<2 \varepsilon_{0}$ where the constant $\varepsilon_{0}$ is given by Lemma 5.9. Let $t \geq \frac{\theta}{100}$ and $(x, t) \in S$. We get with (A) and (D) (see page 64)

$$
\beta_{1 ; k}^{P_{0}}(x, t) \leq\left(\frac{5}{t}\right)^{n+1} \beta_{1 ; k}^{P_{0}}(0,5) \leq\left(\frac{500}{\theta}\right)^{n+1} 2 \varepsilon .
$$

Furthermore, we have with Lemma 6.3 (vi) that $\beta_{1 ; k}^{P_{(x, t)}}(x, t) \leq 2 \varepsilon$ and with $(x, t) \in S \subset S_{\text {total }}$ we obtain $\delta(B(x, t)) \geq \frac{\delta}{2}$. Now, with Lemma $5.9\left(y=x, c=1, \xi=2\left(\frac{500}{\theta}\right)^{n+1}, t_{x}=t_{y}=t\right.$, $\left.\lambda=\frac{\delta}{2}\right)$, there exists some constant $C_{3}=C_{3}\left(N, n, C_{0}, \theta\right)$ so that $\Varangle\left(P_{(x, t)}, P_{0}\right) \leq C_{3} \varepsilon$.

Lemma 8.10. Let $\theta, \alpha>0$. If $k \geq 400$, there exists some constant $\varepsilon^{*}=\varepsilon^{*}\left(N, n, C_{0}, \alpha, \theta\right)$ so that, if $\eta<2 \varepsilon^{*}$, we have for all $\varepsilon \in\left[\frac{\eta}{2}, \varepsilon^{*}\right)$ that for all $x \in F_{3}$ we have $h(x)<\frac{\theta}{100}$. 
Proof. Let $\theta, \alpha>0$ and $k \geq 400$. We set $\varepsilon^{*}:=\min \left\{\bar{\varepsilon}, \varepsilon_{0}, \frac{\alpha}{2 C}\right\}$ where $\bar{\varepsilon}$ is given by Lemma 6.6 and $\varepsilon_{0}$ as well as $C$ are given by Lemma 8.9. Let $\eta \leq 2 \varepsilon<2 \varepsilon^{*}$ and $x \in F_{3}$. With Lemma 6.3 (iv), we have $(x, h(x)) \in S$ and, with Lemma 6.6, we get $\Varangle\left(P_{(x, h(x))}, P_{0}\right)>\frac{1}{2} \alpha$. Hence we obtain $h(x)<\frac{\theta}{100}$ with Lemma 8.9 .

Lemma 8.11. Let $p=2$. There exists some $\hat{k} \geq 400$, some $\tilde{\alpha}=\tilde{\alpha}(n)>0$ and some $\hat{\theta}=\hat{\theta}\left(N, n, C_{0}\right) \in(0,1)$ so that for all $\alpha \in(0, \tilde{\alpha}]$ and $\theta \in(0, \hat{\theta}]$ there exists some $\hat{\varepsilon}=$ $\hat{\varepsilon}\left(N, n, C_{0}, \alpha, \theta\right)$ so that, if $k \geq \hat{k}$ and $\eta<\hat{\varepsilon}^{2}$, we have for all $\varepsilon \in[\sqrt{\eta}, \hat{\varepsilon})$ that there exists some set $H_{\theta} \subset U_{6}$ and some constant $C=C\left(N, n, \mathcal{K}, C_{0}, k\right)$ with $\mathcal{H}^{n}\left(U_{6} \backslash H_{\theta}\right)<C\left(\frac{\varepsilon}{\theta^{n+1} \alpha}\right)^{2}$ and, for all $x \in F_{3} \cap \tilde{\tilde{F}}$, we have $d\left(\pi(x), H_{\theta}\right)>h(x)$.

Proof. Let $\tilde{k}$ and $\tilde{\alpha}(n)$ be the thresholds given by Theorem 7.17 and let $\hat{C}=\hat{C}(N, n)$ be the constant given by Theorem 7.3. Moreover, let $C_{1}=C_{1}\left(N, n, C_{0}\right)$ and $C_{2}=C_{2}\left(N, n, C_{0}\right)$ be the constants given by Corollary 5.3 applied with $\lambda=\frac{\delta}{4}$, and $\delta=\delta(N, n)$ is the value fixed on page 65 . We set $\hat{\theta}:=\frac{1}{400}\left[18 n\left(10^{n}+1\right)\left(\frac{C_{1}}{4}\right)^{n+1} \hat{C}\right]^{-1}$ and choose $\theta \in(0, \hat{\theta}]$. Let $\alpha \in(0, \tilde{\alpha}]$, and let $\bar{\varepsilon}_{1}=\bar{\varepsilon}\left(N, n, C_{0}, \alpha\right), \bar{\varepsilon}_{2}=\bar{\varepsilon}\left(N, n, C_{0}, \alpha\right), \tilde{\varepsilon}=\tilde{\varepsilon}\left(N, n, C_{0}, \alpha\right), \varepsilon_{0}=\varepsilon_{0}\left(N, n, C_{0}, \theta\right)$, $\varepsilon^{*}=\varepsilon^{*}\left(N, n, C_{0}, \alpha, \theta\right)$ be the thresholds given by Lemma 6.6, 6.25, Theorem 7.17, Lemma 8.9 and Lemma 8.10 respectively. Finally, let $C$ be the constant from Lemma 8.9. We set

$$
\hat{\varepsilon}:=\min \left\{\bar{\varepsilon}_{1}, \bar{\varepsilon}_{2}, \tilde{\varepsilon}, \varepsilon_{0}, \varepsilon^{*},(\hat{C} \theta \alpha)^{2}, \frac{\alpha}{400}\left[4 n\left(10^{n}+1\right)\left(\frac{C_{1}}{4}\right)^{n+1} C_{2}\right]^{-1}, \frac{\alpha}{100 C}\right\}
$$

and assume that $k \geq \hat{k}:=\max \{\tilde{k}, 400\}$ and $\eta \leq \hat{\varepsilon}^{2}$. Now let $\varepsilon>0$ with $\eta \leq \varepsilon^{2}<\hat{\varepsilon}^{2}$.

Until now, we defined the map $A$ only on $U_{12}=B(0,12) \cap P_{0}$ (see page 78). Furthermore, we have shown that $A$ is Lipschitz continuous with Lipschitz constant $3 \alpha$ (see Lemma 6.28 on page 84). With Lemma A.8, an application of Kirszbraun's Theorem, there exists an extension $\tilde{A}$ of $A$ with

1. $\tilde{A}: P_{0} \rightarrow \mathbb{R}^{N}$,

2. $\tilde{A}$ has compact support,

3. $\operatorname{Lip}_{\tilde{A}}=\operatorname{Lip}_{A}=3 \alpha$,

4. $A=\tilde{A}$ on $U_{12}$.

If one wants to omit Zorn's lemma, used for the proof of Lemma A.8, one can get the same result with a slightly larger Lipschitz constant (see the remark after Lemma A.8 for details). We denote this extension of $A$ also by $A$.

Using Theorem 7.3 with $g=A, p=2$ and Theorem 7.17, there exist some set $H_{\theta} \subset U_{6}$ and some constant $C=C\left(N, n, \mathcal{K}, C_{0}, k\right)$ with

$$
\mathcal{H}^{n}\left(U_{6} \backslash H_{\theta}\right) \leq \frac{C(n)}{\theta^{2(n+1)} \operatorname{Lip}_{A}^{2}} C \varepsilon^{2} .
$$


Furthermore, we get for all $y \in P_{0}$ some affine map $a_{y}: P_{0} \rightarrow P_{0}^{\perp}$ so that, if $r \leq \theta$ and $B(y, r) \cap H_{\theta} \neq \emptyset$, we have

$$
\left\|A-a_{y}\right\|_{L^{\infty}\left(B(y, r) \cap P_{0}, P_{0}^{\perp}\right)} \leq \hat{C} r \theta \operatorname{Lip}_{A} .
$$

We recall that $\operatorname{Lip}_{A}=3 \alpha$ (cf. Lemma 6.28). For $x \in F_{3} \cap \tilde{\tilde{F}} \subset F_{3} \cap \tilde{F}$, we have with the previous lemma that $h(x)<\frac{\theta}{100}$. Let $t \in\left[h(x), \frac{\theta}{100}\right]$. With Lemma 6.11 , we get $d(x) \leq h(x) \leq t$ and so for all $x^{\prime} \in B(x, 2 t) \cap \tilde{F}$ we obtain by definition of $\tilde{F}$

$$
d\left(x^{\prime}, \pi\left(x^{\prime}\right)+A\left(\pi\left(x^{\prime}\right)\right)\right) \leq \varepsilon^{\frac{1}{2}} d\left(x^{\prime}\right) \leq \varepsilon^{\frac{1}{2}}\left(d(x)+d\left(x, x^{\prime}\right)\right) \leq 3 \varepsilon^{\frac{1}{2}} t .
$$

Let $P_{\pi(x)}$ denote the $n$-dimensional plane, which is the graph of the affine map $a_{\pi(x)}$. Now we assume, contrary to the statement of this lemma, that $d\left(\pi(x), H_{\theta}\right) \leq h(x)$, which implies $\pi(B(x, 2 t)) \cap H_{\theta} \neq \emptyset$, and so, for all $x^{\prime} \in B(x, 2 t) \cap \tilde{F}$, we have with $(8.23)(r=2 t, y=\pi(x))$

$$
d\left(\pi\left(x^{\prime}\right)+A\left(\pi\left(x^{\prime}\right)\right), P_{\pi(x)}\right) \leq d\left(\pi\left(x^{\prime}\right)+A\left(\pi\left(x^{\prime}\right)\right), \pi\left(x^{\prime}\right)+a_{\pi(x)}\left(\pi\left(x^{\prime}\right)\right)\right) \stackrel{(8.23)}{\leq} 6 \hat{C} \theta \alpha t .
$$

We combine those estimates and obtain using $3 \varepsilon^{\frac{1}{2}} \leq 3 \hat{C} \theta \alpha$

$$
d\left(x^{\prime}, P_{\pi(x)}\right) \leq d\left(x^{\prime}, \pi\left(x^{\prime}\right)+A\left(\pi\left(x^{\prime}\right)\right)\right)+d\left(\pi\left(x^{\prime}\right)+A\left(\pi\left(x^{\prime}\right)\right), P_{\pi(x)}\right) \leq 9 \hat{C} \theta \alpha t .
$$

Since $h(x) \underset{\sim}{\leq}$, we get $(x, t) \in S \subset S_{\text {total }}$ with Lemma 6.3 (iv) so that we have $\delta(B(x, t)) \geq \frac{\delta}{2}$. Using $x \in \tilde{\tilde{F}}$ implies

$$
\delta(\tilde{F} \cap B(x, t))=\frac{\mu(\tilde{F} \cap B(x, t))}{t^{n}} \geq \frac{99}{100} \delta(B(x, t))>\frac{1}{4} \delta .
$$

Now we apply Corollary $5.3\left(\Upsilon=\tilde{F}, \lambda=\frac{\delta}{4}\right)$, and so there exist constants $C_{1}\left(N, n, C_{0}\right)$, $C_{2}\left(N, n, C_{0}\right)$ and an $\left(n, 10 n \frac{t}{C_{1}}\right)$-simplex $T=\Delta\left(x_{0}, \ldots, x_{n}\right) \in F \cap B(x, t) \cap \tilde{F}$ so that for all $i \in\{0, \ldots, n\}$

$$
\mu(\underbrace{B\left(x_{i}, \frac{t}{C_{1}}\right) \cap B(x, t) \cap \tilde{F}}_{=: \tilde{B}_{i}}) \geq \frac{t^{n}}{C_{2}} .
$$

With $(x, t) \in S \subset S_{\text {total }}$, we get for all $i \in\{0, \ldots, n\}$

$$
\frac{1}{\mu\left(\tilde{B}_{i}\right)} \int_{\tilde{B}_{i}} d\left(z, P_{(x, t)}\right) \mathrm{d} \mu(z) \leq C_{2} t \frac{1}{t^{n}} \int_{B(x, k t)} \frac{d\left(z, P_{(x, t)}\right)}{t} \mathrm{~d} \mu(z)=C_{2} t \beta_{1 ; k}^{P_{(x, t)}}(x, t) \leq 2 C_{2} t \varepsilon .
$$

This implies for all $i \in\{0, \ldots, n\}$ the existence of $y_{i} \in \tilde{B}_{i}$ with $d\left(y_{i}, P_{(x, t)}\right) \leq 2 C_{2} t \varepsilon$. With Lemma 2.14, we deduce that $S:=\Delta\left(y_{0}, \ldots, y_{n}\right) \subset B(x, t)$ is an $\left(n, 8 n \frac{t}{C_{1}}\right)$-simplex. Next, we apply Lemma $2.28\left(m=n, C=\frac{C_{1}}{8 n}, \hat{C}=1, \sigma=2 C_{2} \varepsilon\right)$ and get

$$
\Varangle\left(P_{(x, t)}, P_{y_{0}, \ldots, y_{n}}\right) \leq 4 n\left(10^{n}+1\right)\left(n \frac{C_{1}}{8 n}\left(2 n \frac{C_{1}}{8 n}\right)^{n}\right) 2 C_{2} \varepsilon \leq \frac{\alpha}{400} .
$$


We have $y_{i} \in \tilde{B}_{i} \subset B(x, 2 t) \cap \tilde{F}$ and hence we get with (8.24) and Lemma $2.28\left(C=\frac{C_{1}}{8 n}\right.$, $\hat{C}=1, \sigma=9 \hat{C} \theta \alpha)$

$$
\Varangle\left(P_{y_{0}, \ldots, y_{n}}, P_{\pi(x)}\right) \leq 4 n\left(10^{n}+1\right)\left(n \frac{C_{1}}{8 n}\left(2 n \frac{C_{1}}{8 n}\right)^{n}\right) 9 \hat{C} \theta \alpha \leq \frac{\alpha}{400} .
$$

With (8.25), we conclude $\Varangle\left(P_{(x, t)}, P_{\pi(x)}\right) \leq \frac{\alpha}{400}+\frac{\alpha}{400}=\frac{\alpha}{200}$, which is true for all $x \in F_{3} \cap \tilde{\tilde{F}}$ with $d\left(\pi(x), H_{\theta}\right) \leq h(x)$ and all $t \in\left[h(x), \frac{\theta}{100}\right]$. Now we use this result for $t=h(x)$ and for $t=\frac{\theta}{100}$ hence we obtain $\Varangle\left(P_{(x, h(x))}, P_{\pi(x)}\right) \leq \frac{\alpha}{200}$ and $\Varangle\left(P_{\left(x, \frac{\theta}{100}\right)}, P_{\pi(x)}\right) \leq \frac{\alpha}{200}$. We conclude $\Varangle\left(P_{(x, h(x))}, P_{\left(x, \frac{\theta}{100}\right)}\right) \leq \frac{\alpha}{100}$. Furthermore, we obtain from Lemma 8.9 that $\Varangle\left(P_{\left(x, \frac{\theta}{100}\right)}, P_{0}\right) \leq$ $\frac{\alpha}{100}$, which implies $\Varangle\left(P_{(x, h(x))}, P_{0}\right) \leq \frac{\alpha}{50}$. This is in contradiction to Lemma 6.6 hence our assumption that $d\left(\pi(x), H_{\theta}\right) \leq h(x)$ is invalid for all $x \in F_{3} \cap \tilde{\tilde{F}}$.

Theorem 8.12. Let $p=2$. There exists some constants $\overline{\bar{k}} \geq 4,0<\overline{\bar{\alpha}}=\overline{\bar{\alpha}}(n)<\frac{1}{6}$ and $0<\overline{\bar{\theta}}=\overline{\bar{\theta}}\left(N, n, C_{0}\right)$ so that, for all $\alpha \in(0, \overline{\bar{\alpha}}]$ and all $\theta \in(0, \overline{\bar{\theta}}]$, there exists some $0<\overline{\bar{\varepsilon}}=$ $\overline{\bar{\varepsilon}}\left(N, n, C_{0}, \alpha, \theta\right)<\frac{1}{8}$ so that, if $k \geq \overline{\bar{k}}$ and $\eta<\bar{\varepsilon}^{2}$, we obtain for all $\varepsilon \in[\sqrt{\eta}, \overline{\bar{\varepsilon}})$

$$
\mu\left(F_{3}\right) \leq 10^{-6} \text {. }
$$

Proof. Let $\overline{\bar{k}}$ be the maximum and $\overline{\bar{\alpha}}<\frac{1}{6}$ be the minimum of all thresholds for $k$ and $\alpha$ given by Lemma $6.28,8.8,8.10$ and 8.11. Furthermore, we set $\overline{\bar{\theta}}:=\hat{\theta}$, where $\hat{\theta}=\hat{\theta}\left(N, n, C_{0}\right)$ is given by Lemma 8.11. Let $0<\alpha \leq \overline{\bar{\alpha}}$ and $0<\theta \leq \overline{\bar{\theta}}$. We define $\overline{\bar{\varepsilon}}=\overline{\bar{\varepsilon}}\left(N, n, C_{0}, \alpha, \theta\right)$ as the minimum of $\frac{1}{16}$, a small constant depending on $N, n, \mathcal{K}, C_{0}, \alpha, \theta$ given by the last lines of this proof, and of all upper bounds for $\varepsilon$ stated in Lemma 6.28, 8.8, 8.10 and 8.11. Let $k \geq \overline{\bar{k}}$ and $\eta \leq \varepsilon^{2}<\bar{\varepsilon}^{2}$. We have $\mu\left(F_{3}\right) \leq \mu\left(F_{3} \cap \tilde{\tilde{F}}\right)+\mu\left(F_{3} \backslash \tilde{\tilde{F}}\right)$. With Lemma $8.8(p=2)$, there exists some constant $C=C\left(N, n, \mathcal{K}, C_{0}\right)$ so that $\mu\left(F_{3} \backslash \tilde{\tilde{F}}\right) \leq \mu(F \backslash \tilde{\tilde{F}}) \leq C \varepsilon^{\frac{1}{2}}$. Hence we only have to consider $\mu\left(F_{3} \cap \tilde{\tilde{F}}\right)$. We set

$$
\left.\mathcal{G}:=\left\{B(x, 2 h(x)) \mid x \in F_{3} \cap \tilde{\tilde{F}}\right)\right\} .
$$

This is a set of nondegenerate balls because $x \in F_{3} \subset F \backslash \mathcal{Z}$. Furthermore, we have $h(x) \leq 50$ for all $x \in F$ (see Definition of $h$ on page 65). With Besicovitch's covering theorem A.12 there exist $N_{0}$ families $\mathcal{B}_{l} \subset \mathcal{G}, l=1, \ldots, N_{0}$, of disjoint balls with

$$
F_{3} \cap \tilde{\tilde{F}} \subset \bigcup_{l=1}^{N_{0}} \bigcup_{B \in \mathcal{B}_{l}} B \cap \tilde{\tilde{F}} .
$$

Now we conclude with property (B) from page 64

$$
\mu\left(F_{3} \cap \tilde{\tilde{F}}\right) \leq \sum_{l=1}^{N_{0}} \sum_{B \in \mathcal{B}_{l}} \mu(B \cap \tilde{\tilde{F}}) \stackrel{(\mathrm{B})}{\leq} C_{0} \sum_{l=1}^{N_{0}} \sum_{B \in \mathcal{B}_{l}}(\operatorname{diam} B)^{n} .
$$

Let $1 \leq l \leq N_{0}$ and let $B_{1}=B\left(x_{1}, 2 h\left(x_{1}\right)\right), B_{2}=B\left(x_{2}, 2 h\left(x_{2}\right)\right) \in \mathcal{B}_{l}$ with $B_{1} \neq B_{2}$. Since the balls in $\mathcal{B}_{l}$ are disjoint, we deduce $2 h\left(x_{1}\right)+2 h\left(x_{2}\right) \leq d\left(x_{1}, x_{2}\right)$ and, because of the definition of $\tilde{F}$ and Lemma 6.11 , we get for $i=1,2$

$$
d\left(x_{i}, \pi\left(x_{i}\right)+A\left(\pi\left(x_{i}\right)\right)\right) \leq \varepsilon^{\frac{1}{2}} d\left(x_{i}\right) \leq \varepsilon^{\frac{1}{2}} h\left(x_{i}\right) .
$$


Since $\varepsilon^{\frac{1}{2}}<\frac{1}{4}, \alpha<\frac{1}{6}$ and $A$ is $3 \alpha$ Lipschitz continuous, we obtain

$$
\begin{aligned}
\frac{7}{4}\left(h\left(x_{1}\right)+h\left(x_{2}\right)\right) & <2 h\left(x_{1}\right)+2 h\left(x_{2}\right)-\varepsilon^{\frac{1}{2}} h\left(x_{1}\right)-\varepsilon^{\frac{1}{2}} h\left(x_{2}\right) \\
& \leq d\left(x_{1}, x_{2}\right)-d\left(x_{1}, \pi\left(x_{1}\right)+A\left(\pi\left(x_{1}\right)\right)\right)-d\left(x_{2}, \pi\left(x_{2}\right)+A\left(\pi\left(x_{2}\right)\right)\right) \\
& \leq d\left(\pi\left(x_{1}\right)+A\left(\pi\left(x_{1}\right)\right), \pi\left(x_{2}\right)+A\left(\pi\left(x_{2}\right)\right)\right) \\
& \leq d\left(\pi\left(x_{1}\right), \pi\left(x_{2}\right)\right)+3 \alpha d\left(\pi\left(x_{1}\right), \pi\left(x_{2}\right)\right) \\
& <\frac{3}{2} d\left(\pi\left(x_{1}\right), \pi\left(x_{2}\right)\right) .
\end{aligned}
$$

This implies $h\left(x_{1}\right)+h\left(x_{2}\right)<d\left(\pi\left(x_{1}\right), \pi\left(x_{2}\right)\right)$. Thus $\pi\left(\frac{1}{2} B_{1}\right)$ and $\pi\left(\frac{1}{2} B_{2}\right)$ are disjoint. We have $x_{i} \in\left(\tilde{\tilde{F}} \cap F_{3}\right) \subset F \subset B(0,5)$ for $i=1,2$. With Lemma 8.10, we conclude that $h\left(x_{i}\right) \leq \frac{\theta}{100}<$ $\frac{1}{2}$. This implies $\pi\left(\frac{1}{2} B_{i}\right) \subset U_{6}$. Using Lemma 8.11, there exists some set $H_{\theta} \subset U_{6}$ and some constant $C=C\left(N, n, \mathcal{K}, C_{0}, k\right)$ with $\mathcal{H}^{n}\left(U_{6} \backslash H_{\theta}\right)<C\left(\frac{\varepsilon}{\theta^{n+1} \alpha}\right)^{2}$ so that $d\left(\pi(x), H_{\theta}\right)>h(x)$ for all $x \in F_{3} \cap \tilde{\tilde{F}}$, in particular for $x=x_{i}$. We conclude that $\pi\left(\frac{1}{2} B_{i}\right) \cap H_{\theta}=\emptyset$, so that we can deduce

$$
\sum_{B \in \mathcal{B}_{l}}(\operatorname{diam} B)^{n}=4^{n} \sum_{B \in \mathcal{B}_{l}}\left(\frac{1}{2} \operatorname{diam} \pi\left(\frac{1}{2} B\right)\right)^{n}=4^{n} \sum_{B \in \mathcal{B}_{l}} \frac{1}{\omega_{n}} \mathcal{H}^{n}\left(\pi\left(\frac{1}{2} B\right)\right) \leq \frac{4^{n}}{\omega_{n}} \mathcal{H}^{n}\left(U_{6} \backslash H_{\theta}\right) .
$$

Now we obtain

$$
\mu\left(F_{3} \cap \tilde{\tilde{F}}\right) \leq C_{0} N_{0} \frac{4^{n}}{\omega_{n}} \mathcal{H}^{n}\left(U_{6} \backslash H_{\theta}\right) \leq C\left(\frac{\varepsilon}{\theta^{n+1} \alpha}\right)^{2} .
$$

and we have already shown that $\mu\left(F_{3} \backslash \tilde{\tilde{F}}\right) \leq C \varepsilon^{\frac{1}{2}}$. Using $\varepsilon<\overline{\bar{\varepsilon}}$, we finally get $\mu\left(F_{3}\right)<$ $10^{-6}$. 



\section{Appendix}

\section{A.1 Measuretheoretical statements}

Lemma A.1. Let $E \subset \mathbb{R}^{N}$ be a $\mathcal{H}^{m}$-measurable set with $0<\mathcal{H}^{m}(E)<\infty$, where $m \in \mathbb{N}$. Then $E$ can be divided into two disjoint subsets

$$
E=E_{u} \dot{\cup} E_{r},
$$

where $E_{u}$ is purely m-unrectifiable and $E_{r}$ is m-rectifiable.

Proof. This statement is proven in [Fed69, Thm. 3.3.13]. The idea of the following alternative proof is from [Paj02, Chap. 1, 5.].

Define

$$
M:=\sup _{\substack{F \subset \mathbb{R}^{n} \\ F \text { m-rectifiable }}} \mathcal{H}^{m}(E \cap F) \leq \mathcal{H}^{m}(E)<\infty .
$$

There is a sequence $F_{j}$ of $m$-rectifiable sets with $\mathcal{H}^{m}\left(E \cap F_{j}\right)>M-\frac{1}{j}$. Define

$$
E_{\mathrm{r}}:=E \cap \bigcup_{j=1}^{\infty} F_{j}, \quad \quad E_{\mathrm{u}}:=E \backslash E_{\mathrm{r}} .
$$

We have to check:

1. $E=E_{\mathrm{u}} \dot{\cup} E_{\mathrm{r}}$.

This holds by definition.

2. $E_{\mathrm{r}}$ is $\mathcal{H}^{m}$-measurable.

Since rectifiable sets are $\mathcal{H}^{m}$-measurable and $E$ is $\mathcal{H}^{m}$-measurable, so is $E_{\mathrm{r}}$.

3. $E_{\mathrm{r}}$ is $m$-rectifiable.

$$
E_{\mathrm{r}}=E \cap \bigcup_{j=1}^{\infty} F_{j}=\bigcup_{j=1}^{\infty}\left(E \cap F_{j}\right)
$$

Since $F_{j}$ is rectifiable, there exists a countable family of Lipschitz functions $\left(\gamma_{j, i}\right)_{i \in \mathbb{N}}$ with

$$
\mathcal{H}^{m}\left(F_{j} \backslash \bigcup_{i=1}^{\infty} \gamma_{j, i}\left(\mathbb{R}^{m}\right)\right)=0 \quad \forall j \in \mathbb{N} .
$$


We have

$$
\begin{aligned}
0 & \leq \mathcal{H}^{m}\left(E_{\mathrm{r}} \backslash \bigcup_{j=1}^{\infty} \bigcup_{i=1}^{\infty} \gamma_{j, i}\left(\mathbb{R}^{m}\right)\right) \\
& \leq \mathcal{H}^{m}\left(\bigcup_{l=1}^{\infty} F_{l} \backslash \bigcup_{j=1}^{\infty} \bigcup_{i=1}^{\infty} \gamma_{j, i}\left(\mathbb{R}^{m}\right)\right) \\
& \leq \mathcal{H}^{m}\left(\bigcup_{l=1}^{\infty}\left(F_{l} \backslash \bigcup_{i=1}^{\infty} \gamma_{l, i}\left(\mathbb{R}^{m}\right)\right)\right) \\
& \leq \sum_{l=1}^{\infty} \mathcal{H}^{m}\left(F_{l} \backslash \bigcup_{i=1}^{\infty} \gamma_{l, i}\left(\mathbb{R}^{m}\right)\right) \\
& =0 .
\end{aligned}
$$

So $E_{\mathrm{r}}$ is rectifiable.

In addition this implies that the countable union of $m$-rectifiable sets is $m$-rectifiable.

4. $E_{\mathrm{u}}$ is purely $m$-unrectifiable.

It holds that $\mathcal{H}^{m}\left(E_{\mathrm{r}}\right)=M$ because $\bigcup_{j=1}^{\infty} F_{j}$ is $m$-rectifiable and, for all $j \in \mathbb{N}$, we have

$$
M \geq \mathcal{H}^{m}\left(E \cap \bigcup_{l=1}^{\infty} F_{l}\right)=\mathcal{H}^{m}\left(E_{\mathrm{r}}\right) \geq \mathcal{H}^{m}\left(E \cap F_{j}\right)>M-\frac{1}{j}
$$

Now let $\gamma: \mathbb{R}^{m} \rightarrow \mathbb{R}^{n}$ be an arbitrary Lipschitz function. Since $\left(\gamma\left(\mathbb{R}^{m}\right) \cup \bigcup_{j=1}^{\infty} F_{j}\right)$ is rectifiable and $E_{\mathrm{r}}$ is $\mathcal{H}^{m}$-measurable, we have

$$
\begin{aligned}
M & \geq \mathcal{H}^{m}\left(E \cap\left(\gamma\left(\mathbb{R}^{m}\right) \cup \bigcup_{j=1}^{\infty} F_{j}\right)\right) \\
& =\mathcal{H}^{m}\left(\left(E \cap \gamma\left(\mathbb{R}^{m}\right)\right) \cup\left(E \cap \bigcup_{j=1}^{\infty} F_{j}\right)\right) \\
& =\mathcal{H}^{m}\left(\left(\left(E_{\mathrm{u}} \dot{\cup} E_{\mathrm{r}}\right) \cap \gamma\left(\mathbb{R}^{m}\right)\right) \cup E_{\mathrm{r}}\right) \\
& =\mathcal{H}^{m}\left(\left(\left(E_{\mathrm{u}} \cap \gamma\left(\mathbb{R}^{m}\right)\right) \dot{\cup}\left(E_{\mathrm{r}} \cap \gamma\left(\mathbb{R}^{m}\right)\right)\right) \cup E_{\mathrm{r}}\right) \\
& =\mathcal{H}^{m}\left(\left(\left(E_{\mathrm{u}} \cap \gamma\left(\mathbb{R}^{m}\right)\right) \dot{\cup} E_{\mathrm{r}}\right)\right. \\
& =\mathcal{H}^{m}\left(\left(E_{\mathrm{u}} \cap \gamma\left(\mathbb{R}^{m}\right)\right)\right)+\mathcal{H}^{m}\left(E_{\mathrm{r}}\right) \\
& \geq 0+M=M .
\end{aligned}
$$


Hence $M=\mathcal{H}^{m}\left(\left(E_{\mathrm{u}} \cap \gamma(\mathbb{R})\right)\right)+M$ and it follows

$$
\mathcal{H}^{m}\left(\left(E_{\mathrm{u}} \cap \gamma(\mathbb{R})\right)\right)=0 .
$$

So $E_{\mathrm{u}}$ is purely $m$-unrectifiable.

Lemma A.2. Let $\mu$ be some outer measure on the $\mathbb{R}$-vector space $X, A \subset X$ some $\mu$ measurable set, $a>0, b \in X, f: a A+b \rightarrow[0, \infty]$ some $\mu \mathrm{L}(a A+b)$-integrable function, and let $\mu(a B+b)=h(a) \mu(B)$ for all $\mu$-measurable sets $B \subset X$, where $h:(0, \infty) \rightarrow(0, \infty)$. Then we have

$$
\int_{a A+b} f(x) \mathrm{d} \mu(x)=h(a) \int_{A} f(a x+b) \mathrm{d} \mu(x) .
$$

Proof. The terminology used in this proof is from [EG92, 1.3].

At first, we prove the statement for simple functions. Let $g: X \rightarrow[0, \infty]$ be some simple function. Then $\bar{g}: X \rightarrow[0, \infty], x \mapsto g(a x+b)$ is some simple function as well. If $y \in[0, \infty]$, we have

$$
\frac{1}{a}\left(g^{-1}(\{y\})-b\right)=\left\{\frac{1}{a}(x-b) \in X \mid g(x)=y\right\}=\{z \in X \mid g(a z+b)=y\}=\bar{g}^{-1}(\{y\}) .
$$

We set

$$
\begin{aligned}
& \mu_{1}:=\mu \mathrm{L} A, \\
& \mu_{2}:=\mu \mathrm{L}(a A+b)
\end{aligned}
$$

and obtain

$$
\begin{aligned}
\int_{a A+b} g(x) \mathrm{d} \mu(x) & =\int g(x) \mathrm{d} \mu_{2}(x) \\
& =\sum_{0 \leq y \leq \infty} y \mu_{2}\left(g^{-1}(\{y\})\right) \\
& =\sum_{0 \leq y \leq \infty} y \mu\left(g^{-1}(\{y\}) \cap(a A+b)\right) \\
& =\sum_{0 \leq y \leq \infty} y h(a) \mu\left(\frac{1}{a}\left(g^{-1}(\{y\})-b\right) \cap A\right) \\
& =h(a) \sum_{0 \leq y \leq \infty} y \mu\left(\bar{g}^{-1}(\{y\}) \cap A\right) \\
& =h(a) \sum_{0 \leq y \leq \infty} y \mu_{1}\left(\bar{g}^{-1}(\{y\})\right) \\
& =h(a) \int \bar{g}(x) \mathrm{d} \mu_{1}(x) \\
& =h(a) \int g(a x+b) \mathrm{d} \mu_{1}(x)
\end{aligned}
$$




$$
=h(a) \int_{A} g(a x+b) \mathrm{d} \mu(x) .
$$

Let $f: a A+b \rightarrow[0, \infty]$ be some $\mu \mathrm{L}(a A+b)$-integrable function. We conclude the following equivalence

$g$ simple, $\mu_{2}$-integrable function with $g \geq f \mu_{2}$-almost everywhere

$\Leftrightarrow g$ simple function, $\int g^{-} \mathrm{d} \mu_{2}<\infty$ or $\int g^{+} \mathrm{d} \mu_{2}<\infty$,

with $g(x) \geq f(x)$ for $\mu$-almost all $x \in a A+b$

$\stackrel{(\text { A.26) }}{\Leftrightarrow} g$ simple function, $h(a) \int \bar{g}^{-} \mathrm{d} \mu_{1}<\infty$ or $h(a) \int \bar{g}^{+} \mathrm{d} \mu_{1}<\infty$,

with $g(a z+b) \geq f(a z+b)$ for $\mu$-almost all $z \in A$

$\Leftrightarrow \bar{g}$ simple, $\mu_{1}$-integrable function with $\bar{g} \geq \bar{f} \mu_{1}$-almost everywhere.

Let $\bar{f}: A \rightarrow[0, \infty], x \mapsto f(a x+b)$. We deduce

$$
\begin{aligned}
\int_{a A+b} f(x) \mathrm{d} \mu(x) & =\int f(x) \mathrm{d} \mu_{2}(x) \\
& =\int^{*} f(x) \mathrm{d} \mu_{2}(x) \\
& =\inf \left\{\int g \mathrm{~d} \mu_{2} \mid g \text { simple, } \mu_{2} \text {-integrable fct. with } g \geq f \mu_{2} \text {-a.e. }\right\} \\
& =\inf \left\{h(a) \int \bar{g} \mathrm{~d} \mu_{1} \mid g \text { simple, } \mu_{2} \text {-integrable fct. with } g \geq f \mu_{2} \text {-a.e. }\right\} \\
& =h(a) \inf \left\{\int \bar{g} \mathrm{~d} \mu_{1} \mid \bar{g} \text { simple., } \mu_{1} \text {-integrable fct. with } \bar{g} \geq \bar{f} \mu_{1} \text {-a.e. }\right\} \\
& =h(a) \int^{*} \bar{f}(x) \mathrm{d} \mu_{1}(x)
\end{aligned}
$$

and analogously we obtain

$$
\int_{a A+b} f(x) \mathrm{d} \mu(x)=\int f(x) \mathrm{d} \mu_{2}(x)=\int_{*} f(x) \mathrm{d} \mu_{2}(x)=h(a) \int_{*} \bar{f}(x) \mathrm{d} \mu_{1}(x) .
$$

This implies

$$
h(a) \int^{*} \bar{f}(x) \mathrm{d} \mu_{1}(x)=\int_{a A+b} f(x) \mathrm{d} \mu(x)=h(a) \int_{*} \bar{f}(x) \mathrm{d} \mu_{1}(x)
$$

and hence $\bar{f}$ is $\mu_{1}$-integrable and we get

$$
\begin{aligned}
\int_{a A+b} f(x) \mathrm{d} \mu(x) & =h(a) \int^{*} \bar{f}(x) \mathrm{d} \mu_{1}(x) \\
& =h(a) \int \bar{f}(x) \mathrm{d} \mu_{1}(x)=h(a) \int_{A} f(a x+b) \mathrm{d} \mu(x) .
\end{aligned}
$$


Lemma A.3. Let $\mu$ be some measure on the $\mathbb{R}$-vectorspace $X$ and let $a>0$. Then

$$
a \mu: A \mapsto a \mu(A)
$$

is some measure on $X$ as well and, if $f: X \rightarrow[0, \infty]$ is some $\mu$-integrable function, we have

$$
a \int f \mathrm{~d} \mu=\int f \mathrm{~d} a \mu .
$$

Proof. At first, we prove that $a \mu$ is some measure on $X$ (cf. [Fal86, 1.1]). Let $\mathcal{M}(\mu)$ be the set of all $\mu$-measurable sets. Then we have

$$
(a \mu)(\emptyset)=a(\mu(\emptyset))=a 0=0
$$

and, if $A_{i} \in \mathcal{M}(\mu), i \in \mathbb{N}$, with $A_{i} \cap A_{j}=\emptyset$ for $i \neq j$, we obtain

$$
(a \mu)\left(\bigcup_{i=1}^{\infty} A_{i}\right)=a\left(\mu\left(\bigcup_{i=1}^{\infty} A_{i}\right)\right)=a\left(\sum_{i=1}^{\infty} \mu\left(A_{i}\right)\right)=\sum_{i=1}^{\infty} a \mu\left(A_{i}\right)=\sum_{i=1}^{\infty}(a \mu)\left(A_{i}\right) .
$$

Hence $a \mu$ is some measure on $X$.

The statement is only proven for simple functions. The adaption to integrable function can be done in the same way as in the previous proof. Let $g: X \rightarrow[0, \infty]$ be some simple function. We have

$$
\begin{aligned}
\int g(x) \mathrm{d} a \mu(x) & =\sum_{0 \leq y \leq \infty} y(a \mu)\left(g^{-1}(\{y\})\right) \\
& =\sum_{0 \leq y \leq \infty} y a \mu\left(g^{-1}(\{y\})\right) \\
& =a \sum_{0 \leq y \leq \infty} y \mu\left(g^{-1}(\{y\})\right) \\
& =a \int g(x) \mathrm{d} \mu(x) .
\end{aligned}
$$

Lemma A.4. Let $\mathcal{E}$ be a set of disjoint balls (open or closed) in $\mathbb{R}^{N}$ with radius equal or larger then $s \in(0, \infty)$ and $B \subset B(x, r)$ for all $B \in \mathcal{E}$. Then $\mathcal{E}$ is a finite set with $\# \mathcal{E} \leq\left(\frac{r}{s}\right)^{N}$. Proof. Choose $l$ different balls $B_{l} \in \mathcal{E}$ and let $\omega_{n}$ be the volume of the $N$-dimensional unit sphere. We have

$$
\begin{aligned}
l s^{N} \omega_{n} & =\sum_{i=1}^{l} \omega_{n} s^{N} \leq \sum_{i=1}^{l} \mathcal{L}^{N}\left(B_{i}\right) \\
& =\mathcal{L}^{N}\left(\bigcup_{i=1}^{l} B_{i}\right) \leq \mathcal{L}^{N}(B(x, r)) \\
& =\omega_{n} r^{N} .
\end{aligned}
$$

This implies $l \leq\left(\frac{r}{s}\right)^{N}$ and hence $\# \mathcal{E} \leq\left(\frac{r}{s}\right)^{N}$. 
Lemma A.5. Let $s>0$ and $B(x, r)$ be an open or closed ball in $\mathbb{R}^{m}$ with $s<r$. There exists some family $\mathcal{E}$ of disjoint closed balls with

(i) $\operatorname{diam} B=2 s$ for all $B \in \mathcal{E}$,

(ii) $B(x, r) \subset \bigcup_{B \in \mathcal{E}} 5 B$,

(iii) $\# \mathcal{E} \leq\left(\frac{2 r}{s}\right)^{m}$,

(iv) for all $B(y, s) \in \mathcal{E}$, we have $y \in B(x, r)$.

Proof. Set $\mathcal{F}=\{B(y, s) \mid y \in B(x, r)\}$. With Vitali's covering theorem [EG92, 1.5.1, Thm 1] there exists a countable family $\mathcal{E}$ of disjoint balls in $\mathcal{F}$ such that

$$
B(x, r) \subset \bigcup_{B \in \mathcal{F}} \subset \bigcup_{B \in \mathcal{E}} 5 B .
$$

Due to $s<r$, we get $B \subset B(x, 2 r)$ for all $B \in \mathcal{E}$. With Lemma A.4, we obtain $\# \mathcal{E} \leq$ $\left(\frac{2 r}{s}\right)^{m}$

Lemma A.6. Let $A \subset \mathbb{R}^{N}$ be a closed set with $\nu(A)>0$, where $\nu$ is some outer measure on $\mathbb{R}^{n}$. There exists some $x \in A$ so that $\nu(B(x, h))>0$ for all $h>0$.

Proof. Assume that there exists some $h>0$ so that, for all $y \in A$, we have $\nu(B(y, h))=0$. We are able to find a countable set $D \subset A$ so that $A \subset \bigcup_{y \in D} B(y, h)$. We have $\nu(A) \leq$ $\sum_{y \in D} \nu(B(y, h))=0$. This is in contradiction, so, for every $h>0$, there exists some $y \in A$ with $\nu(B(y, h))>0$.

Now, for every $i \in \mathbb{N}$, we get some $x_{i} \in A$ with $\nu\left(B\left(x_{i}, \frac{1}{i}\right)\right)>0$. Since $A$ is closed, there exists some subsequence $x_{i}$ and some $x \in A$ so that $\lim _{i \rightarrow \infty} x_{i}=x$.

Let $h>0$. With $i$ small enough, we obtain $\nu(B(x, h)) \geq \nu\left(B\left(x_{i}, \frac{1}{i}\right)\right)>0$.

Lemma A.7. Let $R$ be an $n$-dimensional cube in $\mathbb{R}^{N}$. Then

$$
(\operatorname{diam} R)^{n}=(\sqrt{n})^{n} \mathcal{H}^{n}(R) .
$$

Proof. Let $a$ be the side length of $R$. Then $\operatorname{diam} R=\sqrt{n} a$ and so we get

$$
\mathcal{H}^{n}(R)=a^{n}=\left(\frac{\operatorname{diam} R}{\sqrt{n}}\right)^{n},
$$

which gives the statement.

Lemma A.8. Let $K \subset \mathbb{R}^{m}$ be a bounded set and $f: K \rightarrow \mathbb{R}^{N}$ be a Lipschitz function. Then $f$ has a Lipschitz extension $g: \mathbb{R}^{m} \rightarrow \mathbb{R}^{N}$ with compact support and the same Lipschitz constant.

Remark. Instead of Kirszbraun's Theorem [Fed69, Thm 2.10.43], we can use some simpler theorem for the proof [EG92, 3.1.1, Thm 1] and get the same result but with the larger Lipschitz constant $\operatorname{Lip}_{g}=\sqrt{N} \operatorname{Lip}_{f}$. 
Proof. Let $\operatorname{Lip}_{f}$ be the Lipschitz constant of $f . K$ is bounded, so there exists some ball $B(z, t)$ with $K \subset B(z, t)$. We define

$$
T:=t+\frac{1}{\operatorname{Lip}_{f}} \max _{x \in K}|f(x)| \quad \text { and } \quad \bar{f}(x):= \begin{cases}f(x) & \text { if } x \in K \\ 0 & \text { if } x \in \mathbb{R}^{m} \backslash B(z, T) .\end{cases}
$$

Now we show that $\bar{f}:\left(\mathbb{R}^{m} \backslash B(z, T)\right) \cup K \rightarrow \mathbb{R}^{N}$ is Lipschitz continuous with the same Lipschitz constant as $f$. We only have to prove this for $a \in \mathbb{R}^{m} \backslash B(z, T)$ and $b \in K$

$$
|\bar{f}(a)-\bar{f}(b)|=|f(b)| \leq \operatorname{Lip}_{f}(T-t) \leq \operatorname{Lip}_{f}|a-b| .
$$

By applying Kirszbraun's Theorem [Fed69, Thm 2.10.43] on $\bar{f}$, we obtain a Lipschitz extension $g: \mathbb{R}^{m} \rightarrow \mathbb{R}^{N}$ with compact support and the Lipschitz constant $\operatorname{Lip}_{f}$.

Lemma A.9. Let $(X, T, \phi)$ be a topological Borel measure space where $T$ is the topology and $\phi$ is the Borel measure. If $Q \subset X$ is a $\phi$-measurable set and if $\tilde{f}: Q \rightarrow[0, \infty)$ is a continuous map, then the function $f: X \rightarrow[0, \infty), f(x)=\left\{\begin{array}{ll}\tilde{f}(x) & \text { if } x \in Q \\ 0 & \text { if } x \in X \backslash Q\end{array}\right.$ is $\phi$-measurable on $X$.

Proof. The function $\tilde{f}$ is continuous on $Q$, so $\tilde{f}$ is $\phi \mathrm{L} Q$-measurable. This implies that $\tilde{f}^{-1}(A)$ is $\phi \mathrm{L} Q$-measurable for all open sets $A \subset[0, \infty)$, which is equivalent to $\tilde{f}^{-1}(A) \cap Q$ being $\phi$-measurable for all open sets $A \subset[0, \infty)$. Now let $A \subset[0, \infty)$ be some open set.

1. Case: $0 \notin A$.

We conclude that $f^{-1}(A)=\tilde{f}^{-1}(A) \cap Q$ is $\phi$-measurable.

2. Case: $0 \in A$.

We have that $f^{-1}(A)=f^{-1}(\{0\}) \cup f^{-1}(A \backslash\{0\})$. Now we get

$$
\begin{aligned}
f^{-1}(A) & =\left(f^{-1}(A) \cap Q\right) \cup\left(f^{-1}(A) \backslash Q\right) \\
& =\left(\tilde{f}^{-1}(A) \cap Q\right) \cup \underbrace{\left(f^{-1}(\{0\}) \backslash Q\right)}_{=X \backslash Q} \cup \underbrace{\left(f^{-1}(A \backslash\{0\}) \backslash Q\right)}_{=\emptyset},
\end{aligned}
$$

where the RHS is $\phi$-measurable.

All in all, $f^{-1}(A)$ is $\phi$-measurable for all open sets $A \subset[0, \infty)$, which implies that $f$ is $\phi$-measurable on $X$.

Lemma A.10. Let $\phi_{1}$ be some outer measure on $X_{1}, \phi_{2}$ be some outer measure on $X_{2}$ and $f: X_{1} \rightarrow \mathbb{R}$ be a $\phi_{1}$-measurable function. Then the function $\tilde{f}: X_{1} \times X_{2} \rightarrow \mathbb{R},\left(x_{1}, x_{2}\right) \mapsto f\left(x_{1}\right)$ is $\left(\phi_{1} \times \phi_{2}\right)$-measurable.

Proof. Let $U \subset \mathbb{R}$ be some open set. Then $f^{-1}(U)$ is $\phi_{1}$-measurable and hence we get using Fubini's theorem [EG92, 1.4, Thm. 1] that $\tilde{f}^{-1}(U)=f^{-1}(U) \times X_{2}$ is $\left(\phi_{1} \times \phi_{2}\right)$-measurable, which proves the lemma.

Lemma A.11. For $i \in\{1,2\}$, let $\left(X_{i}, T_{i}, \mu_{i}, \mathcal{M}_{i}\right)$ be a separable topological measure spaces where $T_{i}$ is the topology, $\mu_{i}$ the measure and $\mathcal{M}_{i}$ the set of $\mu_{i}$-measurable sets. If $\mu_{i}$ are Borel measures on $X_{i}$, then the product measure $\mu_{1} \times \mu_{2}$ is a Borel measure on $X_{1} \times X_{2}$ with the usual product topology. 
Proof. Since the $\mu_{1} \times \mu_{2}$-measurable sets are a $\sigma$-algebra, we only have to prove that the open sets are $\mu_{1} \times \mu_{2}$-measurable. Let $E$ be some open set in $X_{1} \times X_{2}$. We show that $E$ is a countable union of measurable sets. The set $T_{1} \times T_{2}:=\left\{M \subset X_{1} \times X_{2} \mid \exists A \in T_{1}, \exists B \in\right.$ $T_{2}$ with $\left.M=A \times B\right\}$ gives a basis of the product space $X_{1} \times X_{2}$. Hence there exists some index set $I$ and sets $A_{i} \times B_{i} \in T_{1} \times T_{2}$ so that $E=\bigcup_{i \in I} A_{i} \times B_{i}$. Since $X_{1}, X_{2}$ are separable, there exist countable bases $\mathcal{B}_{1}, \mathcal{B}_{2}$ of the topologies $T_{1}, T_{2}$. Hence, for every $i \in I$, we find sets $A_{i, j} \in \mathcal{B}_{1}$ and $B_{i, k} \in \mathcal{B}_{2}$, where $j, k \in \mathbb{N}$, so that

$$
E=\bigcup_{i \in I} A_{i} \times B_{i}=\bigcup_{i \in I}\left(\bigcup_{j \in \mathbb{N}} A_{i, j}\right) \times\left(\bigcup_{k \in \mathbb{N}} B_{i, k}\right)=\bigcup_{\substack{i \in I \\ j, k \in \mathbb{N}}} A_{i, j} \times B_{i, k} .
$$

Since the sets $\mathcal{B}_{1}, \mathcal{B}_{2}$ are countable, the set $\mathcal{B}_{1} \times \mathcal{B}_{2}$ is countable, which implies that the last union in the equation above is a countable union. Due to the definition of the product measure and the fact that the measures $\mu_{1}, \mu_{2}$ are Borel measures, all sets in $\mathcal{B}_{1} \times \mathcal{B}_{2}$ are $\mu_{1} \times \mu_{2}$-measurable. So $E$ is measurable as a union of measurable sets.

For an easier verification, we cite Besicovitch's covering theorem [EG92, 1.5.2, Thm. 2].

Theorem A.12 (Besicovitch's covering theorem). There exists a constant $N_{0}$, depending only on $N$, with the following property: If $\mathcal{F}$ is any collection of nondegenerate closed balls in $\mathbb{R}^{N}$ with

$$
\sup \{\operatorname{diam} B \mid B \in \mathcal{F}\}<\infty
$$

and if $A$ is the set of centres of balls in $\mathcal{F}$, then there exists $\mathcal{G}_{1}, \ldots, \mathcal{G}_{N_{0}} \subset \mathcal{F}$ such that each $\mathcal{G}_{i}\left(i=1, \ldots, N_{0}\right)$ is a countable collection of disjoint balls in $\mathcal{F}$ and

$$
A \subset \bigcup_{i=1}^{N_{0}} \bigcup_{B \in \mathcal{G}_{i}} B
$$

\section{A.2 Differentiation and Fourier transform on a linear subspace}

Let $P_{0} \in G(N, n)$ be an $n$-dimensional linear subspace of $\mathbb{R}^{N}$ and $f: P_{0} \rightarrow R$ be some function, where $R \in\left\{\mathbb{R}, \mathbb{R}^{N}\right\}$. In this section, we explain what we mean by differentiating this function and formulating Taylor's theorem in this setting. Furthermore, we define the Fourier transform of $f$ and give some basic properties.

Let $\phi: \mathbb{R}^{n} \rightarrow P_{0}$ be a fixed isometric isomorphism. We set $\tilde{f}: \mathbb{R}^{n} \rightarrow R, \tilde{f}(x)=f(\phi(x))=$ $(f \circ \phi)(x)$.

Definition A.13. Let $l \in \mathbb{N} \cup\{0\}$. We say $f \in C^{l}\left(P_{0}, R\right)$ iff $\tilde{f} \in C^{l}\left(\mathbb{R}^{n}, R\right)$ (l-times continuously differentiable). If $l \geq 1$, we set for all $i \in\{1, \ldots, n\}$

$$
\begin{aligned}
\partial_{i} f & :=D_{i} \tilde{f} \circ \phi^{-1}=D_{i}(f \circ \phi) \circ \phi^{-1}, \\
\Delta f & :=\sum_{j=1}^{n} \partial_{j} \partial_{j} f, \\
D f & :=\left(\partial_{1} f, \ldots, \partial_{n} f\right),
\end{aligned}
$$


and, if $\kappa=\left(\kappa_{1}, \kappa_{2}, \ldots, \kappa_{n}\right)$ is a multi-index, we set

$$
\partial^{\kappa} f:=\partial_{1}^{\kappa_{1}} \partial_{2}^{\kappa_{2}} \ldots \partial_{n}^{\kappa_{n}} f
$$

Furthermore, we use the following notations for $x, y, z \in \mathbb{R}^{n}$ and some multi-index $\kappa$

$$
\begin{aligned}
x & =\left(x_{1}, \ldots, x_{n}\right), \\
x^{\kappa} & =x_{1}^{\kappa_{1}} \cdot x_{2}^{\kappa_{2}} \cdots \cdots x_{n}^{\kappa_{n}}, \\
\kappa ! & =\kappa_{1} ! \kappa_{2} ! \cdots \cdots \kappa_{n} !, \\
|\kappa| & =\kappa_{1}+\cdots+\kappa_{n}, \\
{[y, z]: } & =\{y+t(z-y) \mid t \in[0,1]\} .
\end{aligned}
$$

Lemma A.14. Let $\kappa=\left(\kappa_{1}, \kappa_{2}, \ldots, \kappa_{n}\right)$ be some multi-index with $|\kappa|=l \geq 1$ and $f \in$ $C^{l}\left(P_{0}, \mathbb{R}^{N}\right)$. We have

$$
\partial^{\kappa} f=D^{\kappa} \tilde{f} \circ \phi^{-1}=\left[D^{\kappa}(f \circ \phi)\right] \circ \phi^{-1},
$$

where $D^{\kappa} \tilde{f}=\left(D_{1}\right)^{\kappa_{1}}\left(D_{2}\right)^{\kappa_{2}} \ldots\left(D_{n}\right)^{\kappa_{n}} \tilde{f}$.

Proof. If $|\kappa|=1$, the statement follows directly by definition. We assume that for some $l \in \mathbb{N}$ the statement is true for all multi-indices $\alpha$ with $|\alpha|=l$. Now we choose some multi-index $\kappa$ with $|\kappa|=l+1$. There exists some $i \in\{1, \ldots, n\}$ and some multi-index $\alpha$ with $|\alpha|=l$ so that $\partial^{\kappa} f=\partial_{i} \partial^{\alpha} f$. We have

$$
\begin{aligned}
\partial^{\kappa} f & =\partial_{i} \partial^{\alpha} f \\
& =\partial_{i}\left(D^{\alpha}(f \circ \phi) \circ \phi^{-1}\right) \\
& =D_{i}\left(D^{\alpha}(f \circ \phi) \circ \phi^{-1} \circ \phi\right) \circ \phi^{-1} \\
& =D_{i} D^{\alpha}(f \circ \phi) \circ \phi^{-1} \\
& =D^{\kappa}(f \circ \phi) \circ \phi^{-1} .
\end{aligned}
$$

All in all, we get the statement for all $\kappa$.

Lemma A.15 (Taylor's theorem). Let $f \in C^{s+1}\left(P_{0}, \mathbb{R}^{N}\right)$ and $\left[y_{0}, y\right] \subset P_{0}$. We have

$$
f(y)=p_{s}(y)+R_{s}\left(y-y_{0}\right)
$$

where

$$
p_{s}(y):=\sum_{|\kappa| \leq s} \frac{1}{\kappa !} \partial^{\kappa} f\left(y_{0}\right)\left(\phi^{-1}\left(y-y_{0}\right)\right)^{\kappa}
$$

and

$$
R_{s}\left(y-y_{0}\right):=\int_{0}^{1}(s+1)(1-t)^{s}\left(\sum_{|\kappa|=s+1} \frac{1}{\kappa !} \partial^{\kappa} f\left(y_{0}+t\left(y-y_{0}\right)\right)\left(\phi^{-1}\left(y-y_{0}\right)\right)^{\kappa}\right) \mathrm{d} t .
$$


Proof. Let $\tilde{f}:=f \circ \phi: \mathbb{R}^{n} \rightarrow \mathbb{R}^{N}$, so $\tilde{f} \in C^{s+1}\left(\mathbb{R}^{n}, \mathbb{R}^{N}\right)$, and let $x:=\phi^{-1}(y), x_{0}:=\phi^{-1}\left(y_{0}\right)$. With Taylor's theorem and Lemma A.14, we obtain

$$
\tilde{f}(x)=\tilde{p}_{s}(x)+\tilde{R}_{s}\left(x-x_{0}\right),
$$

where

$$
\begin{aligned}
\tilde{p}_{s}(x) & =\sum_{|\kappa| \leq s} \frac{1}{\kappa !} \underbrace{D^{\kappa} \tilde{f}\left(x_{0}\right)}_{=\left(D^{\kappa} \tilde{f} \circ \phi^{-1}\right)\left(y_{0}\right)}\left(x-x_{0}\right)^{\kappa} \\
& =\sum_{|\kappa| \leq s} \frac{1}{\kappa !} \partial^{\kappa} f\left(y_{0}\right)\left(\phi^{-1}\left(y-y_{0}\right)\right)^{\kappa} \\
& =: p_{s}(y)
\end{aligned}
$$

and

$$
\begin{aligned}
\tilde{R}_{s}\left(x-x_{0}\right) & =\int_{0}^{1}(s+1)(1-t)^{s}\left(\sum_{|\kappa|=s+1} \frac{1}{\kappa !} D^{\kappa} \tilde{f}\left(x_{0}+t\left(x-x_{0}\right)\right)\left(x-x_{0}\right)^{\kappa}\right) \mathrm{d} t \\
& =\int_{0}^{1}(s+1)(1-t)^{s}\left(\sum_{|\kappa|=s+1} \frac{1}{\kappa !} \partial^{\kappa} f\left(y_{0}+t\left(y-y_{0}\right)\right)\left(\phi^{-1}\left(y-y_{0}\right)\right)^{\kappa}\right) \mathrm{d} t \\
& =: R_{s}\left(y-y_{0}\right) .
\end{aligned}
$$

Consequently, we have

$$
f(y)=(f \circ \phi)\left(\phi^{-1}(y)\right)=\tilde{f}(x)=p_{s}(y)+R_{s}\left(y-y_{0}\right) .
$$

Remark A.16 (Transformation from $\mathbb{R}^{n}$ to $P_{0}$ ). Let $\phi: \mathbb{R}^{n} \rightarrow P_{0} \subset \mathbb{R}^{N}$ be an isometric isomorphism, $J_{\phi}(x):=\sqrt{\operatorname{det}\left(D \phi^{T} \cdot D \phi\right)}$ be the Jacobian determinant and let $f \circ \phi$ be an $\mathcal{L}^{n}$-integrable function with $\int_{\mathbb{R}^{n}}|f \circ \phi| \mathrm{d} \mathcal{L}^{n}<\infty$. A consequence of the Area Formula [EG92, 3.3.3, Thm. 2] gives us

$$
\begin{aligned}
\int_{\mathbb{R}^{n}} f(\phi(x)) \mathrm{d} \mathcal{L}^{n}(x) & =\int_{\mathbb{R}^{n}} f(\phi(x)) \underbrace{J_{\phi}(x)}_{=1} \mathrm{~d} \mathcal{L}^{n}(x) \\
& =\int_{\mathbb{R}^{N}}\left[\sum_{x \in \phi^{-1}\{y\}} f(\phi(x))\right] \mathrm{d} \mathcal{H}^{n}(y) \\
& \stackrel{\phi \text { inj. }}{=} \int_{P_{0}} f(y) \mathrm{d} \mathcal{H}^{n}(y) .
\end{aligned}
$$

Lemma A.17 (Partial integration). Let $l \in \mathbb{N}, f \in C^{l}\left(P_{0}, \mathbb{R}^{N}\right)$ and let $\varphi \in C_{0}^{\infty}\left(P_{0}, \mathbb{R}\right)$. Then for all multi-indices $\kappa$ with $|\kappa|=l$

$$
\int_{P_{0}} f(y) \partial^{\kappa} \varphi(y) \mathrm{d} \mathcal{H}^{n}(y)=(-1)^{|\kappa|} \int_{P_{0}} \partial^{\kappa} f(y) \varphi(y) \mathrm{d} \mathcal{H}^{n}(y) .
$$


Proof. Let $\tilde{f}=f \circ \phi$ be defined as above and let $\tilde{\varphi}: \mathbb{R} \rightarrow \mathbb{R}, x \mapsto \varphi(\phi(x))$. With Lemma A.14, we obtain

$$
\left(\partial^{\kappa} \varphi\right)(\phi(x))=\left(\left[D^{\kappa}(\varphi \circ \phi)\right] \circ \phi^{-1}\right)(\phi(x))=\left(D^{\kappa} \tilde{\varphi}\right)(x) .
$$

Now we get with Remark A.16 and partial integration ( $\varphi$ has compact support)

$$
\begin{aligned}
\int_{P_{0}} f(y) \partial^{\kappa} \varphi(y) \mathrm{d} \mathcal{H}^{n}(y) & =\int_{\mathbb{R}^{n}} f(\phi(x))\left(\partial^{\kappa} \varphi\right)(\phi(x)) \mathrm{d} \mathcal{L}^{n}(x)=\int_{\mathbb{R}^{n}} \tilde{f}(x)\left(D^{\kappa} \tilde{\varphi}\right)(x) \mathrm{d} \mathcal{L}^{n}(x) \\
& =(-1)^{|\kappa|} \int_{\mathbb{R}^{n}}\left(D^{\kappa} \tilde{f}\right)(x) \tilde{\varphi}(x) \mathrm{d} \mathcal{L}^{n}(x)=(-1)^{|\kappa|} \int_{P_{0}} \partial^{\kappa} f(y) \varphi(y) \mathrm{d} \mathcal{H}^{n}(y) .
\end{aligned}
$$

Now we define the Fourier transform for some function $f \in \mathscr{S}\left(P_{0}\right)$, where $\mathscr{S}\left(P_{0}\right)$ is the Schwartz space of rapidly decreasing functions $f: P_{0} \rightarrow \mathbb{C}$, cf. [Gra08, 2.2.1 The Class of Schwartz Functions]. We will get the same results as for some function $f \in \mathscr{S}\left(\mathbb{R}^{n}\right)$.

Definition A.18 (Fourier transform). Let $y \in P_{0}$ and $f \in \mathscr{S}\left(P_{0}\right)$. We set

$$
\widehat{f}(y):=\widehat{(f \circ \phi)}\left(\phi^{-1}(y)\right)=\int_{\mathbb{R}^{n}} f(\phi(z)) e^{-2 \pi i \phi^{-1}(y) \cdot z} \mathrm{~d} \mathcal{L}^{n}(z) .
$$

If $f: P_{0} \rightarrow \mathbb{C}^{N}$ with $f_{i} \in \mathscr{S}\left(P_{0}\right)$, i.e., every component of $f$ is a Schwartz function, then we write $f \in \mathscr{S}\left(P_{0}, \mathbb{C}^{N}\right)$. We define the Fourier transform of some function $f \in \mathscr{S}\left(P_{0}, \mathbb{C}^{N}\right)$ by $\widehat{f}:=\left(\widehat{f}_{1}, \ldots, \widehat{f}_{N}\right)$.

Lemma A.19 (Fourier transform and convolution). Let $f, g \in \mathscr{S}\left(P_{0}\right)$ and let the convolution of $f$ and $g$ be defined by $(g * f)(w)=\int_{P_{0}} g(w-v) f(v) \mathrm{d} \mathcal{H}^{n}(v)$. Then we have for $w \in P_{0}$

$$
\widehat{(g * f)}(w)=\widehat{g}(w) \widehat{f}(w) .
$$

Proof. We compute using Remark A.16, Fubini's theorem [EG92, 1.4, Thm. 1] and substitution

$$
\begin{aligned}
\widehat{(g * f)}(w) & =\int_{\mathbb{R}^{n}} \int_{P_{0}} g(\phi(z)-v) f(v) \mathrm{d} \mathcal{H}^{n}(v) e^{-2 \pi i \phi^{-1}(w) \cdot z} \mathrm{~d} \mathcal{L}^{n}(z) \\
& =\int_{\mathbb{R}^{n}} \int_{\mathbb{R}^{n}} g(\phi(z)-\phi(r)) f(\phi(r)) \mathrm{d} \mathcal{L}^{n}(r) e^{-2 \pi i \phi^{-1}(w) \cdot z} \mathrm{~d} \mathcal{L}^{n}(z) \\
& =\int_{\mathbb{R}^{n}} \int_{\mathbb{R}^{n}} g(\phi(z-r)) f(\phi(r)) e^{-2 \pi i \phi^{-1}(w) \cdot z} \mathrm{~d} \mathcal{L}^{n}(z) \mathrm{d} \mathcal{L}^{n}(r) \\
& =\int_{\mathbb{R}^{n}} \int_{\mathbb{R}^{n}} g(\phi(s)) f(\phi(r)) e^{-2 \pi i \phi^{-1}(w) \cdot(s+r)} \mathrm{d} \mathcal{L}^{n}(s) \mathrm{d} \mathcal{L}^{n}(r) \\
& =\int_{\mathbb{R}^{n}} g(\phi(s)) e^{-2 \pi i \phi^{-1}(w) \cdot s} \mathrm{~d} \mathcal{L}^{n}(s) \int_{\mathbb{R}^{n}} f(\phi(r)) e^{-2 \pi i \phi^{-1}(w) \cdot r} \mathrm{~d} \mathcal{L}^{n}(r) \\
& =\widehat{g}(w) \hat{f}(w) .
\end{aligned}
$$


Lemma A.20. Let $f \in \mathscr{S}\left(P_{0}\right), y \in P_{0}, t \in \mathbb{R}$ and set $f_{t}(y):=\frac{1}{t^{n}} f\left(\frac{y}{t}\right)$. We have

$$
\begin{aligned}
\widehat{\left(\partial^{\kappa} f\right)}(y) & =\left(2 \pi i \phi^{-1}(y)\right)^{\kappa} \widehat{f}(y), \\
\widehat{\left(f_{t}\right)}(y) & =\widehat{f}(t y) .
\end{aligned}
$$

Proof. Using Lemma A.14, Definition A.18 and [Gra08, 2.2.11. (9)], we obtain

$$
\begin{aligned}
\widehat{\left(\partial^{\kappa} f\right)}(y) & =\left(\left[D^{\kappa}(f \circ \phi)\right] \circ \phi^{-1}\right) \widehat{(y)} \\
& =\left[D^{\kappa}(f \circ \phi) \hat{]}\left(\phi^{-1}(y)\right)\right. \\
& =\left(2 \pi i \phi^{-1}(x)\right)^{\kappa} \widehat{(f \circ \phi)}\left(\phi^{-1}(y)\right) \\
& =\left(2 \pi i \phi^{-1}(x)\right)^{\kappa} \widehat{f}(y),
\end{aligned}
$$

and, with $t \phi^{-1}(y)=\phi^{-1}(t y)$, we get

$$
\widehat{\left(f_{t}\right)}(y)=\int_{\mathbb{R}^{n}} \frac{1}{t^{n}} f\left(\frac{z}{t}\right) e^{-2 \pi i \phi^{-1}(y) \cdot z} \mathrm{~d} \mathcal{L}^{n}(z)=\int_{\mathbb{R}^{n}} f(v) e^{-2 \pi i \phi^{-1}(y) \cdot t v} \mathrm{~d} \mathcal{L}^{n}(v)=\widehat{f}(t y) .
$$

Lemma A.21. Let $f \in \mathscr{S}\left(P_{0}\right)$ be radial. Then $\widehat{f}$ is radial as well.

Proof. Since $\phi$ is an isometric isomorphism, $f \circ \phi$ is radial. With [Gra08, Cor. 2.2.12], the Fourier transform $\widehat{f \circ \phi}$ is radial and hence $\widehat{f \circ \phi} \circ \phi^{-1}=\widehat{f}$ is radial.

Lemma A.22. Let $f \in \mathscr{S}\left(P_{0}\right)$ be radial. Then $\Delta f$ is radial as well.

Proof. With Lemma A.21, the Fourier transform $\widehat{f}$ is radial. Let $x, y \in P_{0} \subset \mathbb{R}^{N}$ with $|x|=|y|$. We obtain using Lemma A.20

$$
\begin{aligned}
\widehat{\Delta f}(x) & =\sum_{j=1}^{n} \widehat{\partial_{j} \partial_{j} f}(x)=\sum_{j=1}^{n}\left(2 \pi i \phi^{-1}(x)_{j}\right)^{2} \widehat{f}(x) \\
& =(2 \pi i)^{2} \underbrace{\left|\phi^{-1}(x)\right|^{2}}_{=|x|^{2}} \widehat{f}(x)=(2 \pi i)^{2}|y|^{2} \widehat{f}(y)=\widehat{\Delta f}(y) .
\end{aligned}
$$

With Fourier inversion [Gra08, Thm. 2.2.14.], we conclude that $\Delta f$ is radial.

\section{A.3 Littlewood Paley theorem}

The following Lemma is an exercise from [Gra08, Exercise 5.1.4]

Lemma A.23 (Continuous version of the Littlewood Paley theorem). Let $\phi$ be an integrable $C^{1}\left(\mathbb{R}^{n} ; \mathbb{R}\right)$ function with mean value zero fulfilling

$$
|\phi(x)|+|\nabla \phi(x)| \leq C(1+|x|)^{-n-1}
$$


and (using the notation $\phi_{t}(x)=\frac{1}{t^{n}} \phi\left(\frac{x}{t}\right)$ )

$$
0<\int_{0}^{\infty}\left|\widehat{\left(\phi_{t}\right)}(x)\right|^{2} \frac{\mathrm{d} t}{t} \leq c<\infty
$$

for some fixed constant $c$ independent of $x$. For all $q \in(1, \infty)$, there exists some constant $C=C(n, q, \phi)$ such that, for all $f \in L^{q}\left(\mathbb{R}^{n} ; \mathbb{R}^{N}\right)$, we have

$$
\left\|\left(\int_{0}^{\infty}\left|\phi_{t} * f\right|^{2} \frac{\mathrm{d} t}{t}\right)^{\frac{1}{2}}\right\|_{L^{q}\left(\mathbb{R}^{n} ; \mathbb{R}\right)} \leq C\|f\|_{L^{q}\left(\mathbb{R}^{n} ; \mathbb{R}^{N}\right)} .
$$

Proof. The proof is analogue to the proof of the Littlewood-Paley theorem [Gra08, Thm, 5.1.2]. We want to apply [Gra08, Thm. 4.6.1, Banach-Valued Singular Integral Operators] with $r=2, \mathcal{B}_{1}=\mathbb{R}^{N}$ and $\mathcal{B}_{2}=L^{2}((0, \infty), \lambda)$, where $\lambda(A):=\int_{A} 1 \frac{\mathrm{d} t}{t}$ (which implies $\left.\|f\|_{L^{2}((0, \infty), \lambda)}^{2}=\int_{0}^{\infty}|f(t)|^{2} \frac{\mathrm{d} t}{t}\right)$. For $x \in \mathbb{R}^{n} \backslash\{0\}, t>0$ and $a \in \mathcal{B}_{1}$, we set $\vec{K}(x)(a):=\phi_{t}(x) \cdot a$ so that $\vec{K}(x)$ is a bounded linear operator from $\mathcal{B}_{1}$ to $\mathcal{B}_{2}$ and, for $f \in L^{2}\left(\mathbb{R}^{n}, \mathbb{R}^{N}\right)$, we set $\vec{T}(f)(x):=\left(\phi_{t} * f\right)(x) \in L^{2}((0, \infty), \lambda)$.

At first, we prove that $\vec{T}$ is a bounded linear operator from $L^{2}\left(\mathbb{R}^{n} ; \mathcal{B}_{1}\right)$ to $L^{2}\left(\mathbb{R}^{n} ; \mathcal{B}_{2}\right)$. With Fubini's theorem [EG92, 1.4, Thm. 1] and Plancherel's identity [Gra08, Thm. 2.2.14, (4)], we obtain for $f \in L^{2}\left(\mathbb{R}^{n}, \mathbb{R}^{N}\right)$

$$
\begin{aligned}
\|\vec{T}(f)\|_{L^{2}\left(\mathbb{R}^{n} ; \mathcal{B}_{2}\right)}^{2} & =\int_{\mathbb{R}^{n}} \int_{0}^{\infty}\left|\left(\phi_{t} * f\right)(x)\right|^{2} \frac{\mathrm{d} t}{t} \mathrm{~d} x \\
& \left.=\int_{0}^{\infty} \int_{\mathbb{R}^{n}} \mid \widehat{\phi_{t} * f}\right)\left.(x)\right|^{2} \mathrm{~d} x \frac{\mathrm{d} t}{t} \\
& =\int_{0}^{\infty} \int_{\mathbb{R}^{n}}\left|\widehat{\left(\phi_{t}\right.}(x)\right|^{2}|\widehat{f}(x)|^{2} \mathrm{~d} x \frac{\mathrm{d} t}{t} \\
& =\int_{\mathbb{R}^{n}}|\widehat{f}(x)|^{2} \int_{0}^{\infty}\left|\widehat{\left(\phi_{t}\right.}(x)\right|^{2} \frac{\mathrm{d} t}{t} \mathrm{~d} x \\
& \leq C(\phi)\|f\|_{L^{2}\left(\mathbb{R}^{n} ; \mathcal{B}_{1}\right)}^{2}
\end{aligned}
$$

Now we prove that $\vec{K}$ fulfils Hörmander's condition, that means we have to show for $y \in$ $\mathbb{R}^{n} \backslash\{0\}$ that

$$
\int_{|x| \geq 2|y|}\|\vec{K}(x-y)-\vec{K}(x)\|_{\mathcal{B}_{1} \rightarrow \mathcal{B}_{2}} \mathrm{~d} x \leq C<\infty .
$$

Let $|x| \geq 2|y|$ and $\xi \in[0,1]$. This implies

$$
|x-\xi y| \geq|x|-\xi|y| \geq \frac{1}{2}|x| .
$$

Since $\phi \in C^{1}\left(\mathbb{R}^{n} ; \mathbb{R}\right)$, there exists some $\xi \in[0,1]$ with

$$
\begin{aligned}
\left|\phi_{t}(x-y)-\phi_{t}(x)\right| & =\frac{1}{t^{n}}\left|(\nabla \phi)\left(\frac{1}{t}(x-\xi y)\right)\right| \frac{|y|}{t} \\
& \leq \frac{C}{t^{n+1}}\left(1+\frac{1}{2 t}|x|\right)^{-n-1}|y| \\
& \leq \frac{C}{t^{n+1}}|y| .
\end{aligned}
$$


Moreover, we get

$$
\begin{aligned}
\left|\phi_{t}(x-y)-\phi_{t}(x)\right| & \leq \frac{1}{t^{n}}\left|\phi\left(\frac{x-y}{t}\right)\right|+\frac{1}{t^{n}}\left|\phi\left(\frac{x}{t}\right)\right| \\
& \leq \frac{C}{t^{n}}\left(1+\left|\frac{x-y}{t}\right|\right)^{-n-1}+\frac{C}{t^{n}}\left(1+\left|\frac{x}{t}\right|\right)^{-n-1} \\
& \leq \frac{2 C}{t^{n}}\left(1+\frac{1}{2 t}|x|\right)^{-n-1} .
\end{aligned}
$$

We take the geometric mean of A.1 and A.3 and obtain

$$
\begin{aligned}
\left|\phi_{t}(x-y)-\phi_{t}(x)\right| & \leq\left(\frac{C}{t^{n+1}}\left(1+\frac{1}{2 t}|x|\right)^{-n-1}|y| \cdot \frac{2 C}{t^{n}}\left(1+\frac{1}{2 t}|x|\right)^{-n-1}\right)^{\frac{1}{2}} \\
& \leq \frac{2 C}{t^{n+\frac{1}{2}}}|y|^{\frac{1}{2}}\left(1+\frac{1}{2 t}|x|\right)^{-n-1}
\end{aligned}
$$

Now we conclude

$$
\begin{aligned}
& \|\vec{K}(x-y)-\vec{K}(x)\|_{\mathcal{B}_{1} \rightarrow \mathcal{B}_{2}} \\
= & \sup _{\substack{a \in \mathbb{R}^{N} \\
|a|=1}}\left\|\left[\phi_{t}(x-y)-\phi_{t}(x)\right] a\right\|_{L^{2}((0, \infty), \lambda)} \\
= & \left(\int_{0}^{\infty}\left|\phi_{t}(x-y)-\phi_{t}(x)\right|^{2} \frac{\mathrm{d} t}{t}\right)^{\frac{1}{2}} \\
= & \left(\int_{0}^{\frac{|x|}{2}}\left|\phi_{t}(x-y)-\phi_{t}(x)\right|^{2} \frac{\mathrm{d} t}{t}+\int_{\frac{|x|}{2}}^{\infty}\left|\phi_{t}(x-y)-\phi_{t}(x)\right|^{2} \frac{\mathrm{d} t}{t}\right)^{\frac{1}{2}} \\
& \left(\int_{0}^{\frac{|x|}{2}}\left[\frac{2 C}{t^{n+\frac{1}{2}}}|y|^{\frac{1}{2}}\left(1+\frac{1}{2 t}|x|\right)^{-n-1}\right]^{2} \frac{\mathrm{d} t}{t}+\int_{\frac{|x|}{2}}^{\infty}\left[C \frac{1}{t^{n+1}}|y|\right]^{2} \frac{\mathrm{d} t}{t}\right)^{\frac{1}{2}} \\
\leq & 2 C\left(|y| \int_{0}^{\frac{|x|}{2}}\left[\frac{1}{t^{n+\frac{1}{2}}}\left(\frac{2 t}{|x|}\right)^{n+1}\right]^{2} \frac{\mathrm{d} t}{t}+|y|^{2} \int_{\frac{|x|}{2}}^{\infty}\left[\frac{1}{t^{n+1}}\right]^{2} \frac{\mathrm{d} t}{t}\right)^{\frac{1}{2}} \\
\leq & 2 C\left(|y||x|^{-2 n-2} 2^{2 n+2} \int_{0}^{\frac{|x|}{2}} t \frac{\mathrm{d} t}{t}+|y|^{2} \frac{1}{2 n+2}\left(\frac{|x|}{2}\right)^{-2 n-2}\right)^{\frac{1}{2}} \\
\leq & C(n)|y|^{\frac{1}{2}}|x|^{-n-\frac{1}{2}}+C(n)|y||x|^{-n-1} .
\end{aligned}
$$


Finally, we can prove Hörmander's condition

$$
\begin{aligned}
& \int_{|x| \geq 2|y|}\|\vec{K}(x-y)-\vec{K}(x)\|_{\mathcal{B}_{1} \rightarrow \mathcal{B}_{2}} \mathrm{~d} x \\
\leq & C|y|^{\frac{1}{2}} \int_{|x| \geq 2|y|}|x|^{-n-\frac{1}{2}} \mathrm{~d} x+C|y| \int_{|x| \geq 2|y|}|x|^{-n-1} \mathrm{~d} x \\
\leq & C|y|^{\frac{1}{2}} \int_{2|y|}^{\infty} r^{-\frac{3}{2}} \mathrm{~d} r+C|y| \int_{2|y|}^{\infty} r^{-2} \mathrm{~d} r \\
\leq & C|y|^{\frac{1}{2}}|y|^{-\frac{1}{2}}+C|y||y|^{-1}=C(n) .
\end{aligned}
$$

Using [Gra08, Thm. 4.6.1, Banach-Valued Singular Integral Operators] $(p=q)$, for all $1<$ $q<\infty$, there exists some constant $C=C(n, q, \phi)$ so that, for all $f \in L^{q}\left(\mathbb{R}^{n}, \mathbb{R}^{N}\right)$, we obtain

$$
\left\|\left(\int_{0}^{\infty}\left|\phi_{t} * f\right|^{2} \frac{\mathrm{d} t}{t}\right)^{\frac{1}{2}}\right\|_{L^{q}\left(\mathbb{R}^{n} ; \mathbb{R}\right)}=\|\vec{T}(f)\|_{L^{q}\left(\mathbb{R}^{n} ; L^{2}((0, \infty), \lambda)\right)} \leq C\|f\|_{L^{q}\left(\mathbb{R}^{n}, \mathbb{R}^{N}\right)} .
$$





\section{Bibliography}

[AF03] Robert A. Adams and John J. F. Fournier. Sobolev spaces, volume 140 of Pure and Applied Mathematics (Amsterdam). Elsevier/Academic Press, Amsterdam, second edition, 2003.

[Alt06] Hans Wilhelm Alt. Lineare Funktionalanalysis. Springer, Heidelberg, Berlin, New York, fifth edition, 2006.

[BK12] Simon Blatt and Sławomir Kolasiński. Sharp boundedness and regularizing effects of the integral Menger curvature for submanifolds. Adv. Math., 230(3):839-852, 2012 .

[Bla13] Simon Blatt. A note on integral Menger curvature for curves. Math. Nachr., 286(2-3):149-159, 2013.

[DS93] Guy David and Stephen Semmes. Analysis of and on uniformly rectifiable sets, volume 38 of Mathematical Surveys and Monographs. American Mathematical Society, Providence, RI, 1993.

[Dud10] James J. Dudziak. Vitushkin's conjecture for removable sets. Universitext. Springer, New York, 2010.

[EG92] Lawrence C. Evans and Ronald F. Gariepy. Measure theory and fine properties of functions. Studies in advanced mathematics. CRC Press, Boca Raton, 1992.

[Fal86] Kenneth J. Falconer. The geometry of fractal sets, volume 85 of Cambridge Tracts in Mathematics. Cambridge University Press, Cambridge, 1986.

[Fed69] Herbert Federer. Geometric measure theory. Die Grundlehren der mathematischen Wissenschaften, Band 153. Springer-Verlag New York Inc., New York, 1969.

[FHW94] Michael H. Freedman, Zheng-Xu He, and Zhenghan Wang. Möbius energy of knots and unknots. Ann. of Math. (2), 139(1):1-50, 1994.

[Gra08] Loukas Grafakos. Classical Fourier analysis, volume 249 of Graduate Texts in Mathematics. Springer, New York, second edition, 2008.

[Hah05] Immo Hahlomaa. Menger curvature and Lipschitz parametrizations in metric spaces. Fund. Math., 185(2):143-169, 2005.

[Hah07] Immo Hahlomaa. Curvature integral and Lipschitz parametrization in 1-regular metric spaces. Ann. Acad. Sci. Fenn. Math., 32(1):99-123, 2007. 
[Hah08] Immo Hahlomaa. Menger curvature and rectifiability in metric spaces. Adv. Math., 219(6):1894-1915, 2008.

[Jon90] Peter W. Jones. Rectifiable sets and the traveling salesman problem. Invent. Math., 102(1):1-15, 1990.

[Jon91] Peter W. Jones. The traveling salesman problem and harmonic analysis. Publ. Mat., 35(1):259-267, 1991. Conference on Mathematical Analysis (El Escorial, 1989).

[Kol12] Sławomir Kolasiński. Geometric Sobolev-like embedding using high-dimensional Menger-like curvature, 2012. arXiv:1205.4112.

[KSvdM13] Sławomir Kolasiński, Paweł Strzelecki, and Heiko von der Mosel. Characterizing $W^{2, p}$ submanifolds by $p$-integrability of global curvatures. Geom. Funct. Anal., 23(3):937-984, 2013.

[Lég99] Jean-Christophe Léger. Menger curvature and rectifiability. Ann. of Math. (2), 149(3):831-869, 1999.

[LM00] Yong Lin and Pertti Mattila. Menger curvature and $C^{1}$ regularity of fractals. Proc. Amer. Math. Soc., 129(6):1755-1762 (electronic), 2000.

[LW09] Gilad Lerman and J. Tyler Whitehouse. High-dimensional Menger-type curvatures - Part II: $d$-separation and a menagerie of curvatures. Constr. Approx., 30(3):325-360, 2009.

[LW11] Gilad Lerman and J. Tyler Whitehouse. High-dimensional Menger-type curvatures - Part I: Geometric multipoles and multiscale inequalities. Rev. Mat. Iberoam., 27(2):493-555, 2011.

[Mat95] Pertti Mattila. Geometry of sets and measures in Euclidean spaces, volume 44 of Cambridge Studies in Advanced Mathematics. Cambridge University Press, Cambridge, 1995. Fractals and rectifiability.

[Men30] Karl Menger. Untersuchungen über allgemeine Metrik. Math. Ann., 103(1):466$501,1930$.

[NTV14] Fedor Nazarov, Xavier Tolsa, and Alexander Volberg. The Riesz transform, rectifiability, and removability for Lipschitz harmonic functions. Publ. Mat., 58(2):517-532, 2014.

[O'H91] Jun O'Hara. Energy of a knot. Topology, 30(2):241-247, 1991.

[Paj02] Hervé Pajot. Analytic capacity, rectifiability, Menger curvature and the Cauchy integral, volume 1799 of Lecture Notes in Mathematics. Springer-Verlag, Berlin, 2002.

[Sch12] Sebastian Scholtes. For which positive $p$ is the integral Menger curvature $\mathcal{M}_{p}$ finite for all simple polygons?, 2012. arXiv:1202.0504. 
[SSvdM09] Paweł Strzelecki, Marta Szumańska, and Heiko von der Mosel. A geometric curvature double integral of Menger type for space curves. Ann. Acad. Sci. Fenn. Math., 34(1):195-214, 2009.

[SSvdM10] Paweł Strzelecki, Marta Szumańska, and Heiko von der Mosel. Regularizing and self-avoidance effects of integral Menger curvature. Ann. Sc. Norm. Super. Pisa Cl. Sci. (5), 9(1):145-187, 2010.

[SSvdM13] Paweł Strzelecki, Marta Szumańska, and Heiko von der Mosel. On some knot energies involving Menger curvature. Topology Appl., 160(13):1507-1529, 2013.

[Ste66] P. Stein. Classroom Notes: A Note on the Volume of a Simplex. Amer. Math. Monthly, 73(3):299-301, 1966.

[SvdM11] Paweł Strzelecki and Heiko von der Mosel. Integral Menger curvature for surfaces. Adv. Math., 226(3):2233-2304, 2011.

[SvdM13a] Paweł Strzelecki and Heiko von der Mosel. Menger curvature as a knot energy. Phys. Rep., 530(3):257-290, 2013.

[SvdM13b] Paweł Strzelecki and Heiko von der Mosel. Tangent-point repulsive potentials for a class of non-smooth $m$-dimensional sets in $\mathbb{R}^{n}$. Part I: Smoothing and selfavoidance effects. J. Geom. Anal., 23(3):1085-1139, 2013.

[Tol14a] Xavier Tolsa. Analytic capacity, the Cauchy transform, and nonhomogeneous Calderón-Zygmund theory, volume 307 of Progress in Mathematics. Birkhäuser/Springer, Cham, 2014.

[Tol14b] Xavier Tolsa. Rectifiable measures, square functions involving densities, and the cauchy transform, 2014. arXiv:1408.6979.

[TT14] Xavier Tolsa and Tatiana Toro. Rectifiability via a square function and Preiss's theorem, 2014. arXiv:1402.2799.

[Yos80] Kôsaku Yosida. Functional analysis, volume 123 of Grundlehren der Mathematischen Wissenschaften [Fundamental Principles of Mathematical Sciences]. Springer-Verlag, Berlin-New York, sixth edition, 1980. 